\title{
Österreichischer Forschungs- und Technologiebericht 2002
}

Lagebericht gem. §8 FOG über die aus Bundesmitteln geförderte Forschung, Technologie und Innovation in Österreich

Juni 2002 


\section{KURZBERICHT: \\ FORSCHUNGS- UND TECHNOLOGIEPOLITISCHE ENTWICKLUNGEN 2001}

Der vorliegende Forschungs- und Technologiebericht 2002 der beiden für Forschung und Technologie zuständigen Minister enthält eine im wesentlichen auf Indikatoren beruhende Strukturdarstellung des österreichischen Forschungs-, Technologie- und Innovationssystems und positioniert dieses auch im internationalen Vergleich.

Der nächste Forschungs- und Technologiebericht wird 2003 von der Bundesregierung dem Nationalrat vorgelegt. Er wird einen umfassenden Rechenschaftsbericht über die in der Legislaturperiode getroffenen forschungs- und technologiepolitischen Maßnahmen der Bundesregierung enthalten.

Bereits in diesem Jahr soll deshalb ein kurzer Überblick über die wesentlichsten forschungs- und technologiepolitischen Entwicklungen im Berichtsjahr gegeben werden:

\section{Realisierung des Regierungsübereinkommens:}

Im Regierungsübereinkommen 2000 - 2003 wurde der besondere Stellenwert, den wissenschaftliche Forschung und technologische Entwicklung in der Sicherung und Schaffung gesellschaftlichen Wohlstands einnimmt, hervorgehoben. Als Beratungsorgan der Bundesregierung und einzelner Minister steht der unabhängige Rat für Forschung und Technologieentwicklung zur Verfügung, dessen Aufgaben sich erstmals auf sämtliche Fragen der Forschungs- und Technologiepolitik und deren Umsetzung beziehen.

Ziel der Bundesregierung ist es,

- $\quad$ den Anteil der Forschungsausgaben am BIP signifikant anzuheben (bis zum Jahre 2005 auf $2,5 \%$ des BIP),

- den Wirtschaftsstandort Österreich durch zukunftssichernde Investitionen mit wissenschaftlicher Kompetenz anzureichern, um damit nachhaltig dessen Innovations- und Wettbewerbsfähigkeit zu stärken, und

- $\quad$ an gesamteuropäische Entwicklungen in Forschung und Technologie teilzuhaben, um auch im globalen Wettbewerb bestehen zu können.

Zur Zielerreichung der angestrebten Steigerung der F\&E-Quote ist sowohl eine rigorose Steigerung des Mitteleinsatzes der öffentlichen Hand, als auch - in einem überproportionalen Ausmaß - der Wirtschaft erforderlich. Die öffentlichen F\&E-Ausgaben werden daher überwiegend in Bereichen angesetzt, in denen mit ,großer Hebelwirkung“ zu rechnen ist. Eine Optimierung bestehender Strukturen muss die Investitionen komplementär ergänzen.

Im Bundesbudget 2001 wurden für das F\&E-Offensivprogramm 7 Mrd. ATS (508,7 Mio. €) veranschlagt. Im Auftrag der Bundesregierung gibt der Rat für Forschung und Technologieentwicklung Empfehlungen zum Einsatz dieser Sondermittel ab. 


\section{Reformdialoge:}

Der dritte und vierte Reformdialog der Bundesregierung war jeweils dem Thema Forschung, Technologie und Innovation gewidmet und fand unter Einbindung aller politischen Kräfte und involvierten Gruppen am 29. Jänner und am 23. Mai 2001 statt. Damit wurde der Öffentlichkeit ein klares Signal der Prioritätensetzung in offensiven Bereichen wie Bildung, Forschung, Technologie und Innovation gegeben, welche trotz der Budgetkonsolidierung stattfindet. Die Bundesregierung bekannte sich zur deutlichen Erhöhung der Investitionen in Forschung und Technologie: 7 Mrd. ATS Sondermittel für Forschung, Entwicklung und Innovation tragen dazu bei, den Wissenschaftsstandort Österreich zu sichern und die auf know-how aufbauende Wettbewerbsfähigkeit der heimischen Wirtschaft nachhaltig zu verbessern. Der Rat für Forschung und Technologieentwicklung wurde als beratender ,think tank“ für die gezielte und wirksame Umsetzung dieser Ziele geschaffen

In beiden Reformdialogen wurde diskutiert, wie Österreich unter Mitwirkung aller Beteiligten in eine Top-Position geführt werden kann. Der Rat für Forschung und Technologieentwicklung soll deshalb bei der Erfüllung seines Auftrages, eine langfristige österreichische Strategie für den Bereich Forschung und Technologieentwicklung zu erarbeiten, den Dialog mit den Bundesländern, Sozialpartnern und mit den Parlamentariern suchen.

\section{Wichtige Themen des dritten Reformdialogs waren}

- die Absicherung des Wissenschafts- und Wirtschaftsstandortes Österreich auf europäischer Ebene,

- die Optimierung bestehender Strukturen in den Forschungsförderungsfonds, den Universitäten und der Wirtschaft, um Innovation bestmöglich zu fördern,

- $\quad$ das Setzen der richtigen Anreize, um ein stärkeres Engagement der Wirtschaft in forschungsund technologieorientierten Bereichen zu stimulieren.

Im Kompetenzbereich des Bundesministeriums für Bildung, Wissenschaft und Kultur wurden als prioritäre Beiträge identifiziert:

- Förderung des Forschernachwuchses (Ausbildung und Mobilitätsförderung von qualifiziertem Forschernachwuchs),

- Schwerpunktsetzung und Standortbereinigung (Infrastrukturverbesserungen an Universitäten und außeruniversitären Forschungseinrichtungen),

- Gezielte Integration in den Europäischen Forschungsraum

- Einrichtung einer Großforschungseinrichtung in Österreich (gepulste Neutronenspallationsquelle auf Beschleunigerbasis - AUSTRON)

- Bewusstseinsbildung über die Notwendigkeit von Wissenschaft und Forschung

Im Kompetenzbereich des Bundesministeriums für Verkehr, Innovation und Technologie wurden als prioritäre Beiträge identifiziert:

- Strukturänderung mit größtmöglicher Hebelwirkung, um zusätzliche Gelder der Industrie für die Forschung und Entwicklung zu bewirken,

- Verbesserung der Zusammenarbeit zwischen Wirtschaft und Wissenschaft vor allem für den Wissenstransfer und den Technologietransfer,

- Verbesserung der Schnittstellen zur Standortpolitik und größere Vernetzung des österreichischen Innovationssystems durch verstärkte Interaktion und Kooperation, 
- Verbesserung der Ausbildung und frühzeitige Integration der Auszubildenden in den Wirtschaftsprozess besonders im Informations- und Kommunikationstechnologiebereich.

Im 4. Reformdialog für Österreich am 23. Mai 2001 wurde das Strategiepapier des Rates für Forschung und Technologieentwicklung ,2,5\% + plus: Wohlstand durch Forschung und Innovation" der Öffentlichkeit vorgestellt. Das ambitionierte Ziel, bis zum Jahr 2005 eine Forschungsquote von 2,5\% zu erreichen, kann nur durch gemeinsame Anstrengungen aller Mitwirkenden erreicht werden. Es soll daher ein forschungs- und technologiefreundliches Klima gefördert werden, in dem dieses Ziel als gesamtgesellschaftliche Priorität anerkannt wird. Neben dem Einsatz zusätzlicher öffentlicher Mittel werden von der Wirtschaft ebenfalls erhebliche Investitionen in die Forschung geleistet werden müssen, wie die entsprechenden internationalen Vergleiche zeigen (siehe dazu auch Kapitel 2 des Forschungs- und Technologieberichts). Die Anhebung des Forschungsfreibeitrags auf 25\% bzw. 35\% durch die Steuerreform 2000 und die zusätzlich für Forschung und Technologieentwicklung investierten $7 \mathrm{Mrd}$. ATS schaffen eine durch wissenschaftliche Studien belegte positive Ausgangslage.

Forschung und technologische Entwicklung können im internationalen Wettbewerb nur mit einem bestmöglich qualifizierten wissenschaftlichen und technischem Personal bestehen. Daher kommt der wissenschaftlichen Lehre an Universitäten und Fachhochschulen ein besonders hoher Stellwert zu; mittelfristig soll eine Steigerung der Akademikerquote in Österreich auf 20\% erreicht werden. Die bevorstehende Universitätenreform mit den Zielen einer umfassenden Autonomie, der Schaffung klarer Zurechenbarkeit von Entscheidungen und Verantwortung, der Steigerung der Attraktivität und Wettbewerbsfähigkeit der österreichischen Universitäten ist dazu eine der wesentlichen Voraussetzungen.

\section{Verwendung der Sondermittel der Forschungs- und Technologieoffensive:}

\section{Kompetenzbereich BMBWK:}

Bisher wurden Mittel für Forschungsprogramme, Initiativen und Infrastrukturverbesserungen im Bereich des BMBWK in Höhe von ca. 186 Mio. € empfohlen:

- Rd. 31 \% der empfohlenen Mittel wurden bis März 2002 für universitäre Vorhaben im Bereich von Infrastrukturverbesserungen vergeben, die wesentliche Voraussetzung auch für die verstärkte Zusammenarbeit der universitären Grundlagenforschung mit der industriellen Forschung und Entwicklung sind, ca. $13 \%$ an außeruniversitäre Forschungseinrichtungen und Wissenschaftliche Anstalten.

- Das Genomforschungsprogramm GEN-AU, mit welchem die Zukunftsfähigkeit Österreichs auf dem zentralen Feld der Biowissenschaften im 21. Jahrhundert gesichert werden soll, hat zum einen erhebliche Bedeutung für den Erkenntnisfortschritt in der Wissenschaft und zum anderen für die Innovationsfähigkeit einer Vielzahl von Wirtschaftsbereichen mit hoher Wertschöpfung. Damit leistet das bm:bwk einen wichtigen Beitrag zur Umsetzung des Regierungsübereinkommens. Für die Finanzierung des Programms wurden für 3 Jahre ca. $17 \%$ der gesamten bisher empfohlenen Sondermittel bereitgestellt.

- Ca. $15 \%$ wurden für weitere Programme reserviert, die der Internationalisierung und der Steigerung der wissenschaftlichen Leistungsfähigkeit Österreichs im globalen Wettbewerb dienen, 
wie z. B. Beteiligung an CERN- oder Unterstützung von Beteiligungen an EU-Forschungsprogrammen durch Anbahnungs- und Zusatzfinanzierungen.

- Ca. $8 \%$ der Mittel werden für die Förderung des wissenschaftlichen Forschungsnachwuchses entweder durch Aufstockung bereits bestehender Programme, Preise oder Stipendien oder zur Schaffung neuer Möglichkeiten zur Förderung internationaler Kooperation z.B. mit ost- und südostasiatischen Forschungseinrichtungen verwendet.

- $\quad 11 \%$ sind für Kooperationen zwischen Wissenschaft und Wirtschaft vorgesehen, vor allem. in zukunftsweisenden Bereichen wie der Biotechnologie, der Molekularen Medizin oder der Molekularen Bioinformatik (Bsp. ÖZBT, CeMM oder IMBA).

- $\quad$ Rd. $2 \%$ entfallen auf die Neugestaltung der Bund-Bundesländer-Kooperation zum Ausbau und zur Vertiefung der gemeinsamen Forschungsanstrengungen von Bund und Ländern und zur Hebung der Forschungsquote in den Ländern.

- Weitere rd. $2 \%$ entfallen auf die Förderung von Frauen in Forschung und Technologie (FFORTE), sowie auf $\mathrm{IMST}^{2}$, einem Programm zur Weiterentwicklung der Didaktik in Mathematik und Naturwissenschaften.

- Für die Vorbereitungsarbeiten zur Errichtung einer internationalen Großforschungseinrichtung in Österreich (AUSTRON) wurden rd. 0,6 \% der empfohlenen Sondermitteln zur Finanzierung freigegeben.

\section{Kompetenzbereich BMVIT:}

Bisher wurden Mittel für Forschungsprogramme, Initiativen und Infrastrukturverbesserungen im Bereich des BMVIT in Höhe von 225,17 Mio. € empfohlen:

Für thematische Impulsprogramme wurden 30,5 \% der empfohlenen Mittel verwendet. Diese Programme setzen konkrete Maßnahmen in Richtung der anwendungsnahen Spitzenforschung und zwar in folgenden strategischen Bereichen:

○ Nachhaltig Wirtschaften,

○ Intelligente Verkehrssysteme und Services,

- Aeronautik,

○ Nationales Weltraumprogramm,

○ Impulsprogramm Fit für Informationstechnologie FIT-IT,

○ Bewusstseinsbildung für Forschung und Entwicklung.

27,5\% der Mittel wurden für Strukturprogramme zur Verbesserung der Gründungsdynamik besonders der technologieorientierten Unternehmen empfohlen. Dies betrifft auch den Universitäten - spin - off - Bereich:

○ „Kompetenzzentren K Plus“ zur Kooperation zwischen Wissenschaft und Wirtschaft und zur Förderung der exzellenten Forschung, 
○ „Inkubatorenprogramm A plus B“ zur dauerhaften Steigerung der forschungsintensiven und technologieorientierten Existenzgründungen aus dem akademischen Sektor,

○ „Seedfinancing“ zur Frühphasenfinanzierung für technologieorientierte Unternehmen,

○ „Fachhochschule FH Plus“ zum Ausbau der F\&E Infrastruktur an den Fachhochschulen zur Steigerung der Forschung und Entwicklung in Klein und Mittelbetrieben in den Regionen,

○ „REG plus“ zum Ausbau der regionalen Innovations- und Technologiezentren für den Technologietransfer in den Regionen.

Zur kurzfristigen verstärkten Impulssetzung in der wirtschaftsbezogenen und der wissenschaftlichen Forschung wurden dem Forschungsförderungsfonds für die gewerbliche Wirtschaft (FFF) und dem Fonds zur wissenschaftlichen Forschung (FWF) für 2001 und 2002 42\% der empfohlenen Sondermittel zugewiesen.

\section{Europäische Forschungs- und Technologiepolitik:}

Die österreichische Forschungs- und Technologiepolitik ist eingebettet in den Kontext der Europäischen Union. Die Mitteilung der Kommission über "Die internationale Dimension des Europäischen Forschungsraums" vom 25. Juni $2001^{1}$ gibt den Rahmen für die weitere Entwicklung vor.

Mit dem Europäischen Forschungsraum wurde ein neuer politischer Kontext geschaffen, der die Umsetzung einer neuen Strategie für die internationale wissenschaftlich-technische Zusammenarbeit auf der Grundlage der bereits innerhalb der Union erreichten Ergebnisse möglich macht.

Hauptziel dieser Strategie muss es sein,

- den Europäischen Forschungsraum zu einem Anziehungspol und Bezugspunkt für die besten Wissenschaftler zu machen;

- $\quad$ den Forschern und der Industrie den Zugang zu den anderswo in der Welt entwickelten Erkenntnissen und Technologien sowie zu den von der europäischen Forschung benötigten Experimentiereinrichtungen zu ermöglichen;

- wissenschaftliche und technische Aktivitäten im Dienste der Außen- und Entwicklungshilfepolitik der Union zu entwickeln;

- die wissenschaftlichen und technologischen Kapazitäten der Union und der Drittländer für Initiativen zu mobilisieren, die auf weltweite Probleme eingehen, die auch für die Gemeinschaft von Bedeutung sind.

Eine europäische Politik der internationalen wissenschaftlich-technischen Zusammenarbeit erfordert einen langen Atem und muss sich sowohl auf die wirksame Koordinierung der Aktivitäten der Gemeinschaft mit denen der Mitgliedstaaten als auch auf die Schaffung von Synergien zwischen

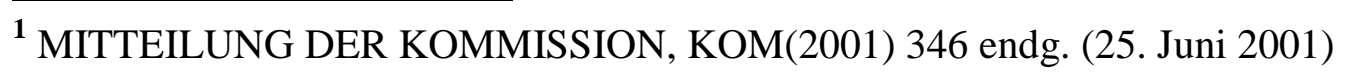


den Gemeinschaftsmaßnahmen im Bereich der Außenbeziehungen und der wissenschaftlichen Forschung und der Technologie stützen.

In der globalen Wissensgesellschaft muss sich eine Politik mit diesem Anspruch entwickeln können und $\mathrm{zu}$ einem wesentlichen Bestandteil der Gemeinschaftspolitik der Forschung und technologischen Entwicklung werden. 
2 DAS ÖSTERREICHISCHE INNOVATIONSSYSTEM IM INTERNATIONALEN

2.1 Allgemeine Trends in den Ausgaben für Forschung und Entwicklung............... 3

2.2 Sektorale Struktur der Finanzierung und Durchführung von

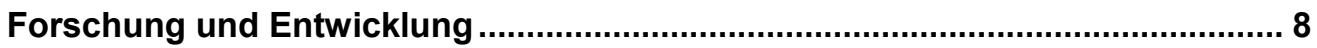

3 GRUNDLAGENFORSCHUNG UND WISSENSCHAFTSSYSTEM................................. 12

3.1 Internationale Trends in der Grundlagenforschung ......................................... 12

3.2 Publikationen österreichischer WissenschafterInnen ....................................... 16

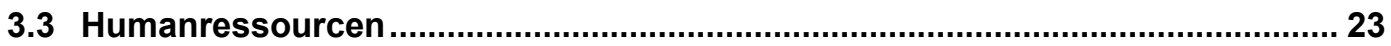

4 DIE INTERNATIONALISIERUNG DES ÖSTERREICHISCHEN FORSCHUNGS-

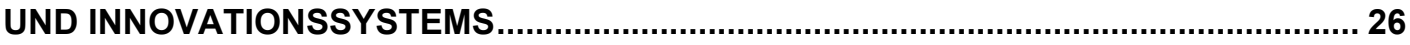

4.1 Die österreichische Beteiligung am 5. Rahmenprogramm ............................... 26

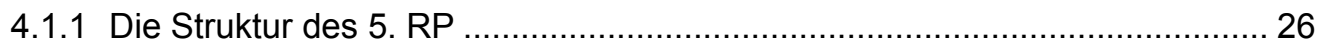

4.1.2 Die österreichische Beteiligung im internationalen Vergleich ...................... 27

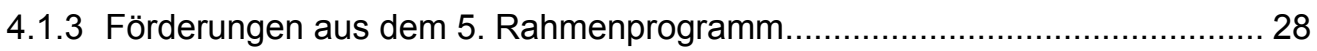

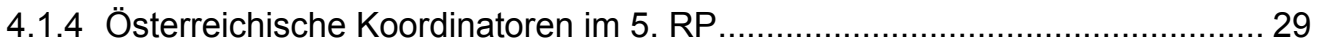

4.1.5 Die österreichische Beteiligung nach Organisationstypen.......................... 30

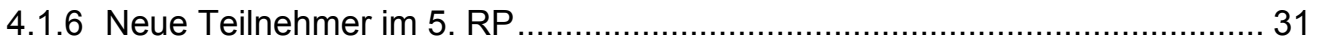

4.2 Die österreichische Beteiligung in EUREKA ….............................................. 34

4.3 Die österreichische Beteiligung an COST ….................................................... 37

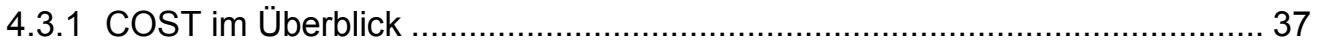

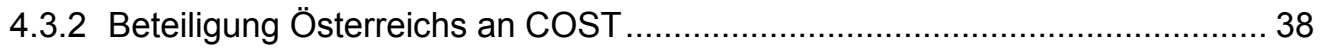

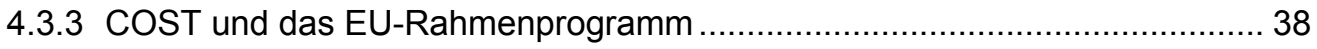

4.4 Österreichs Mitgliedschaft in der European Space Agency (ESA) ................... 39

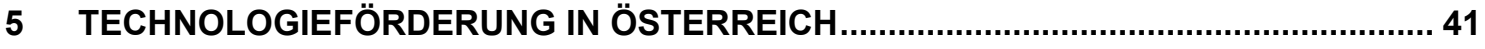

5.1 Direkte Technologieförderung .......................................................................... 41

5.1.1 Die direkte unternehmensbezogene Technologieförderung des

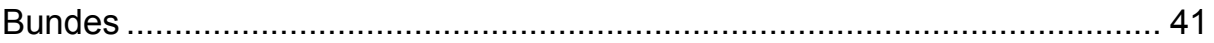

5.1.2 Schwerpunkte der direkten Technologieförderung des Bundes ................... 43

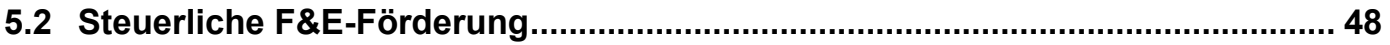

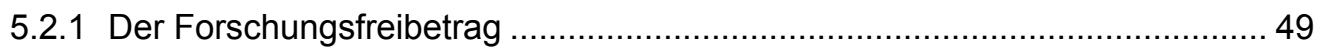

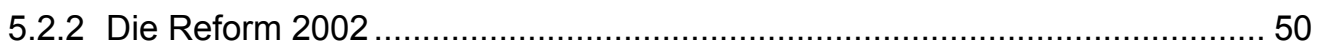

5.2.3 Empirische Evidenz zum Forschungsfreibetrag......................................... 51 
6 ZUR TECHNOLOGISCHEN LEISTUNGSFÄHIGKEIT DES

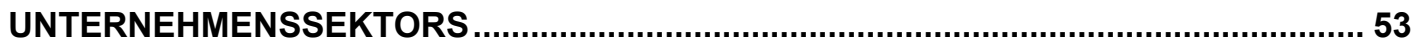

6.1 Die Patentspezialisierung des Unternehmenssektors ...................................... 53

6.2 Forschung, Technologie und Wirtschaftsstruktur............................................. 61

7 INFORMATIONS- UND KOMMUNIKATIONSTECHNOLOGIEN.....................................67

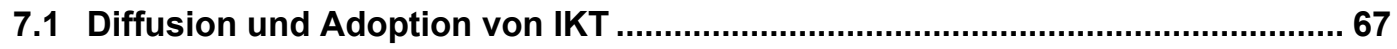

7.2 Die Diffusion von IKT und e-Commerce im Unternehmenssektor ..................... 72

7.3 Die österreichische Politik zur Informationsgesellschaft ................................. 78

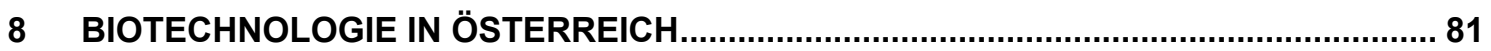

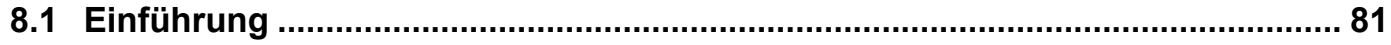

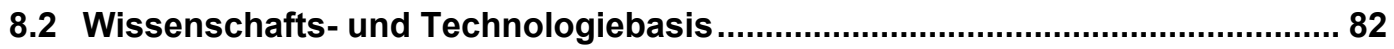

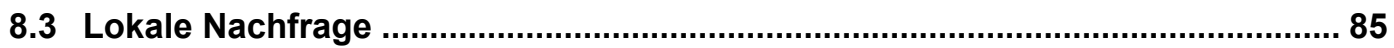

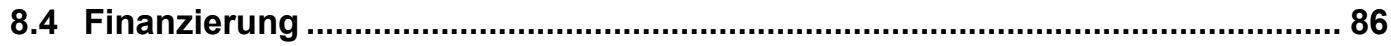

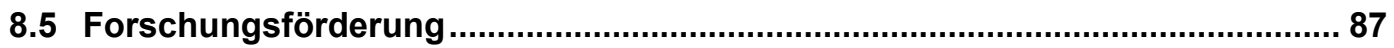

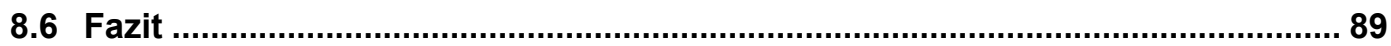

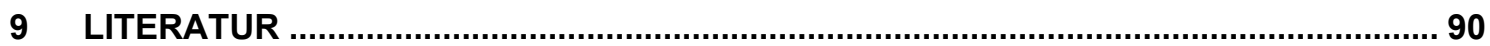

STATISTISCHER ANHANG 


\section{Einleitung}

Der österreichische Forschungs- und Technologiebericht erscheint mit der vorliegenden Ausgabe inzwischen zum vierten Mal. Zum zweiten Mal legen das Bundesministerium für Bildung, Wissenschaft und Kultur (BMBWK) und das Bundesministerium für Verkehr, Technologie und Innovation (BMVIT) einen gemeinsamen Bericht gemäß § 8 FOG vor. An der Intention des vorliegenden Lageberichts, ausgearbeitet im Rahmen des Politikberatungsprogramms TIP ${ }^{1}$, hat sich wenig geändert: die Fundierung der technologie- und forschungspolitischen Debatte mit Hintergrundinformationen, die sowohl die Entwicklung Österreichs anhand zentraler Indikatoren darstellen und interpretieren, als auch die heimische Position im europäischen bzw. OECD-Kontext aufzeigen sollen. Der Bericht wendet sich damit sowohl an Politik und Verwaltung, als auch an die Wirtschaft, Wissenschaft und die interessierte Öffentlichkeit.

Was hat sich nun seit Erscheinen des ersten Technologieberichts geändert? Zunächst haben die meisten Befunde, die in den letzten Jahren zum österreichischen Innovationssystem gemacht wurden, weiterhin Gültigkeit. Weiterhin ist der niedrige Finanzierungsanteil des Unternehmenssektors sowie ein relativ großer Anteil des Hochschulsektors bei den Forschungsausgaben charakteristisch für das österreichische Innovationssystem. Vergleiche mit anderen Industrieländern zeigen jedoch, dass vor allem der Unternehmenssektor für die größte Dynamik bei den F\&E-Ausgaben im letzten Jahrzehnt sorgte. Die vorhandene Industriestruktur in Österreich kann demnach nach wie vor als eine strukturelle Schwäche auf dem Weg zu einer wissensbasierten Gesellschaft interpretiert werden. Dennoch zeigt sich, dass aufgrund der Maßnahmen des öffentlichen Sektors die F\&E-Ouote in den letzten Jahren konstant gestiegen ist und mittlerweile EU-Durchschnitt erreichen konnte.

Der Anteil der Grundlagenforschung an den österreichischen F\&E-Ausgaben liegt mit 17,8\% knapp unter dem OECD-Durchschnitt, wobei diese Aktivitäten vor allem im Hochschulbereich gebündelt sind. Das Wissenschaftssystem erweist sich, was, die Zahl der Publikationen pro Einwohner und ihren Weltanteil betrifft, durchaus vergleichbar mit anderen kleinen Staaten wie Finnland, Dänemark oder Irland. Publikationen, an denen österreichische WissenschafterInnen beteiligt waren, werden vor allem in den naturwissenschaftlichen Fächern und medizinischen Teilfächern häufiger als der Weltdurchschnitt zitiert. Obwohl Österreich relativ hohe Mittel für tertiäre Ausbildung verwendet, ist der Bevölkerungsanteil mit tertiärer Ausbildung allerdings vergleichsweise gering.

Grundlegend verbessert hat sich seit Erscheinen des ersten Technologieberichts dagegen die Einbindung Österreichs in internationale Forschungskooperationen, vor allem in die Rahmenprogramme der EU. In den letzten Jahren konnte eine merklich zunehmende

\footnotetext{
${ }^{1}$ tip (http://www.tip.ac.at/) ist ein Beratungsprogramm für Forschungs- und Technologiepolitik im Auftrag des BMBWK und BMVIT, an dem das Österreichische Institut für Wirtschaftsforschung (WIFO), ARC Seibersdorf research Gmbh (ARCS), Joanneum Research (JR) und Technopolis Österreich beteiligt sind die Autorlnnen dieses Bands sind Bernhard Dachs (ARCS; Koordination), Helmut Gassler (JR), Gernot Hutschenreiter (WIFO), Norbert Knoll (WIFO), Hannes Leo (WIFO), Jörg Mahlich (Technopolis), Doris Schartinger (ARCS), Andreas Schibany (JR) und Gerhard Streicher (JR)
} 
Beteiligung österreichischer Forschungsorganisationen sowie Unternehmen und damit einhergehend auch eine Steigerung der monetären Förderungen beobachtet werden. Besonders erfreulich ist, dass der Anteil der Erstteilnehmer im soeben auslaufenden 5. Rahmenprogramm der EU ausgesprochen hoch ist. Dies deutet auf eine substantielle Zunahme der Bereitschaft, internationale Technologiekooperationen einzugehen, hin. Österreichs Beteiligung an EUREKA und den Aktivitäten der ESA bedeuten weitere Möglichkeiten für heimische Firmen und Universitäten an internationalen Forschungskooperationen teilzunehmen.

Die zunehmende Bedeutung von Forschungs- und Technologiepolitik lässt sich auch daran erkennen, dass sich Technologieförderung inzwischen zum quantitativ bedeutendsten Segment der direkten Wirtschaftsförderung des Bundes entwickelt hat. Der Fokus der Fördermaßnahmen liegt, gemessen an der Anzahl der Förderprojekte sowie an der Summe der Förderbarwerte, auf einer klar erkennbaren Verbesserung der Verbindung zwischen Forschung und Innovation. $80 \%$ der Barwerte werden über unspezifische direkte F\&E-Förderungen vergeben. Der Forschungsfreibetrag wird im internationalen Vergleich in bezug auf die Generosität relativ gut bewertet.

Dagegen bestätigt einer Auswertung von Patentstatistiken die bekannten Aussagen zur technologischen Leistungsfähigkeit des österreichischen Unternehmenssektors: die österreichische Wirtschaft ist vor allem auf Produkte mit mittlerem Technologieniveau spezialisiert, während einige Hochtechnologiesektoren, vor allem Computer und Telekommunikation, unterrepräsentiert sind. Der Bericht zeigt somit, dass Österreichs „Technologielücke" nicht durch eine niedrigere Forschungsintensität alleine erklärt werden kann, sondern aufgrund der vorhandenen Industriestruktur sich die Spezialisierung der österreichischen Wirtschaft auf weniger dynamische Sektoren beschränkt. Nur eine Koppelung von Forschungs- und Technologiepolitik mit einer gezielten Strukturpolitik kann somit die Basis für einen Aufholprozess Österreichs in die Hochtechnologiesektoren schaffen.

Die Diffusion von Informations- und Kommunikationtechnologien ist ein Beispiel für einen gelungenen Aufholprozess Österreichs. Zwar scheinen einzelne nordische Staaten und die USA nach wie vor uneinholbar, im Vergleich zu 1998 hat sich die Position Österreichs jedoch deutlich verbessert. Der Einsatz von business-to-business-Anwendungen im Unternehmenssektor befindet sich noch in einer frühen Phase, die Unternehmen sind jedoch auf diese Technologie bereits gut vorbereitet.

Ein Bereich, dem eine ähnliche Entwicklung gelingen könnte, ist die Biotechnologie. Die Ausgangsbedingungen im wissenschaftlichem und technologischen Bereich sind gut, weshalb eine mit Deutschland vergleichbare Dynamik der Unternehmensgründung erwarten werden kann. 


\section{Das österreichische Innovationssystem im internationalen Vergleich}

\subsection{Allgemeine Trends in den Ausgaben für Forschung und Entwicklung}

In den vergangenen zwei Jahrzehnten hat sich die Ansicht durchgesetzt, dass Wissen eine zentrale Voraussetzung für Wettbewerbsfähigkeit, Wirtschaftswachstum und soziale Entwicklung ist. Wissen zu schaffen, zu verteilen und zu nutzen sind mehr denn je zentrale Fähigkeiten moderner Volkswirtschaften und ihre Förderung eine der wichtigsten Aufgaben der Politik. So sind die gesamtwirtschaftlichen Ausgaben für Forschung und Entwicklung (GERD) und die Forschungsquote, also der Anteil der F\&E-Ausgaben am Bruttoinlandsprodukt, zu zentralen Größen in der politischen Diskussion geworden.

Die gesamtwirtschaftlichen Ausgaben für Forschung und Entwicklung werden 2002 nach neuesten Schätzungen von Statistik Austria 4.217,27 Mio. € erreichen ${ }^{2}$. Wie während der gesamten 90er Jahre werden die F\&E-Ausgaben auch heuer wieder schneller als das Bruttoinlandsprodukt wachsen, wodurch sich die Forschungsquote erneut von 1,91\% (2001) auf 1,95\% im Jahr 2002 erhöhen wird.

\footnotetext{
Die gesamtwirtschaftliche Ausgaben für F\&E werden 2002 4,2 Mrd. € erreichen, was einer F\&E-Quote von 1,95\% entspricht. Treibende Kraft dieser Entwicklung ist der öffentliche Sektor, während - nach den vorliegenden Schätzungen - die Aufwendungen der Unternehmen im Vergleich deutlich langsamer wachsen.
}

Die treibende Kraft hinter der Entwicklung während der letzten Jahre ist, wie in Abbildung 2-1 zu erkennen, eindeutig der öffentliche Sektor. So werden die Ausgaben des Bundes für in Österreich durchgeführte F\&E 2002 rund 1457 Mio. $€$ erreichen, sie liegen damit um rund 7,3\% über dem Vorjahresniveau. Insgesamt sind im Bundesvoranschlag 2001, der in der Schätzung verwendet wurde, 508,7 Mio. $€$ unter dem Titel „Offensivprogramm F\&E“ veranschlagt. Statistik Austria geht von der Annahme aus, dass davon ca. 218 Mio. $€$ im Jahr 2002 ausgegeben werden. Die Inanspruchnahme der restlichen Mittel (ca. 160 Mio. $€$ ) ist für die F\&E-Ausgaben 2003 vorgesehen. Auch die Bundesländer haben ihre Aufwendungen seit 1997 wesentlich erhöht (vgl. statistischer Anhang, Tab. 1).

Dagegen wachsen die F\&E-Aufwendungen des Unternehmenssektors seit 1997 deutlich langsamer als die Anteile des Bundes und der Länder. Somit ist ein Hebeleffekt der

\footnotetext{
Die statistischen Angaben dieses Kapitels stammen von der OECD (Science, Technology and Industry Scoreboard, http://www1.oecd.org/publications/e-book/92-2001-04-1-2987/), $\quad$ EUROSTAT (Strukturindikatoren, http://europa.eu.int/comm/eurostat/Public/datashop/print-product/DE?catalogue=Eurostat\&product=1-ir022$\underline{D E \& m o d e=d o w n l o a d}$ ) sowie Daten, die Statistik Austria für die Erstellung dieses Berichts zur Verfügung gestellt hat.
} 
gestiegenen öffentlichen Aufwendungen, also eine Stimulierung der Ausgaben des Unternehmenssektors durch verstärke öffentliche Ausgaben - zumindest in den Schätzungen noch nicht zu erkennen. Auch der Finanzierungsanteil des Auslands, der seit der zweiten Hälfte der 90er Jahre stark zugenommen hat, ist nach diesen Angaben im Zeitraum 1997/2002 zu Wachstumsraten zurückgekehrt, die unter jenen der öffentlichen Ausgaben liegen.

\section{Abbildung 2-1: Entwicklung der F\&E-Ausgaben und der F\&E-Quote, 1990 - 2002}

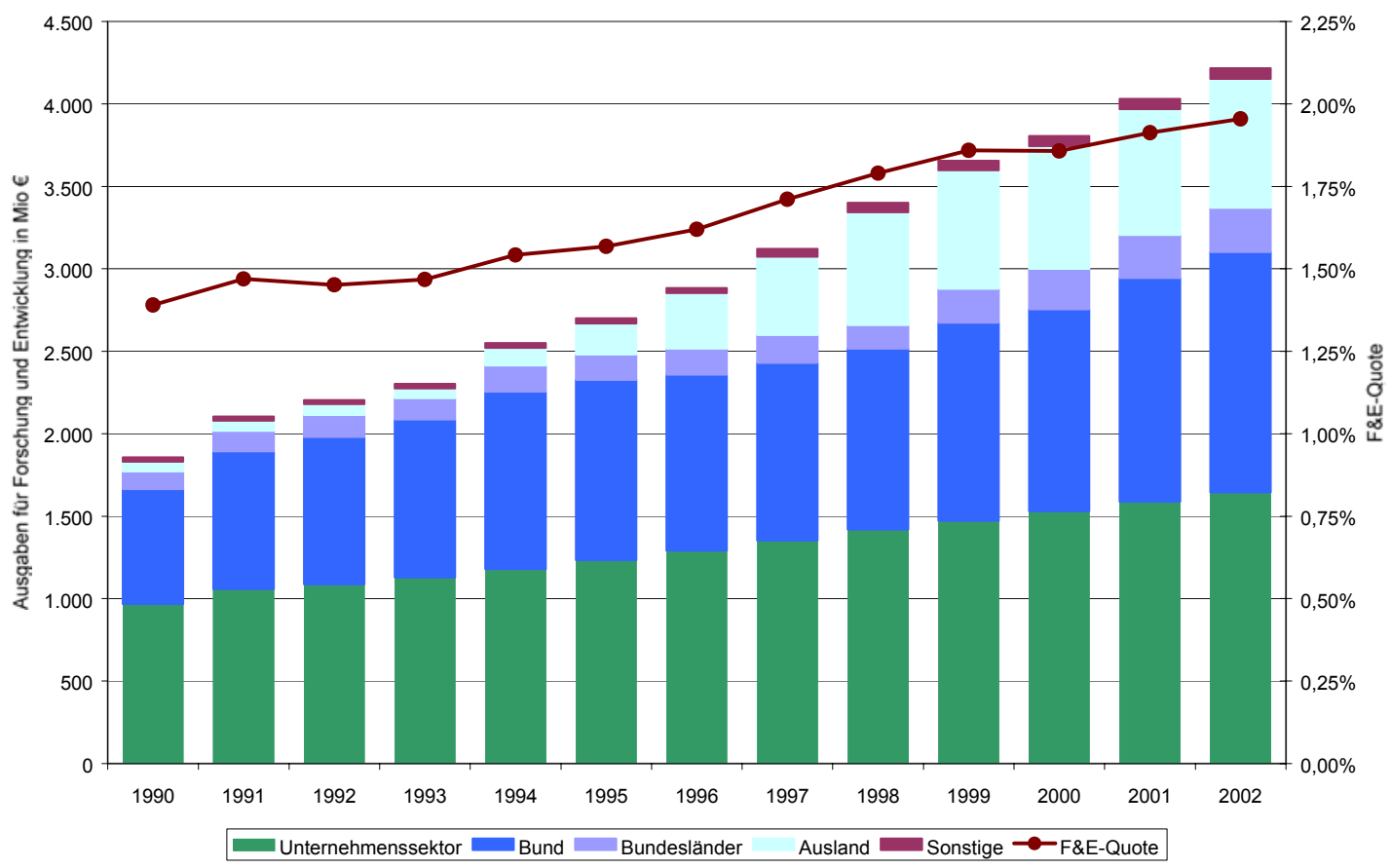

Quelle: Statistik Austria

Österreich befindet sich mit einer aktuellen F\&E-Quote von 1,95\% im Mittelfeld der europäischen Staaten. Da die Werte anderer Länder für 2002 noch nicht verfügbar sind, können derzeit Vergleiche nur auf Basis des Jahres 1999 angestellt werden. Österreich befand sich damals mit einer Forschungsquote von 1,86\% noch knapp unter dem EU-Durchschnitt von $1,92 \%$. Deutlich höher als der österreichische Wert war außerdem der OECD-Vergleichswert von $2,21 \%$, dessen Höhe auf die Ausgabenniveaus der USA $(2,64 \%)$ und Japans $(3,04 \%)$ zurückgeführt werden kann.

Solche Vergleiche sind allerdings nur für Staaten mit ähnlichem Einkommensniveau aussagekräftig, denn allgemein weisen Volkswirtschaften mit höherem Pro-Kopf-Einkommen auch höhere F\&E-Quoten auf. Die Zusammenhänge zwischen den Ausgaben für Forschung und Entwicklung und dem Grad des wirtschaftlichen Wohlstandes eines Landes sind dabei jedoch zu vielfältig, um sie in eine einfache Formel pressen zu können. Wenn also Österreichs internationale Position in Forschung und Entwicklung beurteilt werden soll, scheint nur ein Vergleich mit Ländern, die ein ähnliches Einkommensniveau aufweisen, sinnvoll. Das sind etwa Belgien, die Niederlande, Japan oder Irland (vgl. Abbildung 2-2). 
Abbildung 2-2: F\&E-Quote und BIP pro Kopf für verschiedene OECD-Länder, 1999

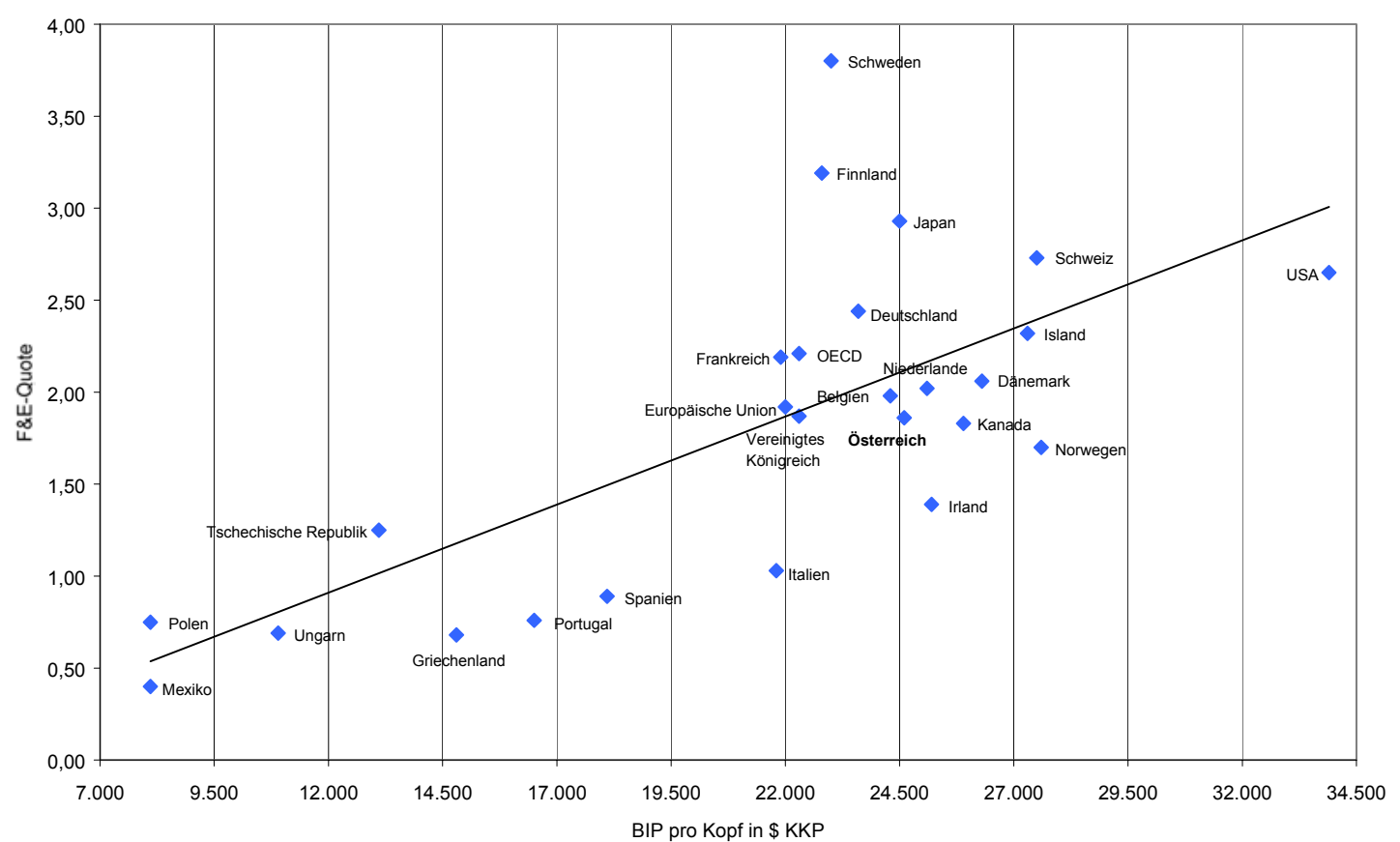

F\&E-Quoten für 1999, mit Ausnahme von Schweiz (1996), Irland, Griechenland, Tschechische Republik (1997), Niederlande (1998)

Quelle: OECD 2001, eigene Berechnungen

Österreich schneidet im Vergleich mit diesen Ländern nur unterdurchschnittlich ab. Wie die Trendlinie zeigt, könnte aufgrund der Wirtschaftskraft Österreichs eine F\&E-Quote von etwa 2,1\% erwartet werden. Aber auch wenn dieser Wert erreicht wird, ist Österreich noch nicht im Spitzenfeld zu finden: Länder wie Finnland, Schweden, Deutschland oder Frankreich geben im Vergleich zu ihrem Einkommensniveau wesentlich mehr für Forschung und Entwicklung als Österreich aus. Dabei sollte aber nicht, wie in Kap. 7 gezeigt wird, auf eine allgemein niedrigere technologische Leistungsfähigkeit der heimischen Industrie, die den Großteil der F\&EAufwendungen aufbringt, geschlossen werden. Ein wichtiger Grund für dieses Ergebnis ist auch die Struktur der österreichischen Wirtschaft.

Obwohl also noch ein Rückstand zu Ländern mit vergleichbarem Entwicklungsniveau besteht, konnte Österreich seinen Abstand während der 90er Jahre merklich verringern. Die Entwicklung der österreichischen F\&E-Ausgaben während der letzten Jahre kann als doppelter Aufholprozess beschrieben werden: Einerseits gegenüber den EU-Ländern, andererseits - als Teil der EU - gegenüber dem OECD-Durchschnitt und den USA. Österreichs Forschungsausgaben haben sich seit Beginn der 90er Jahre nach Angaben der OECD mit einer durchschnittlichen jährlichen Wachstumsrate von $4,7 \%$ wesentlich dynamischer entwickelt als der OECD-Durchschnitt $(2,78 \%)$ und wuchsen mehr als doppelt so schnell wie der EUDurchschnitt (1,62\%). Länder wie Italien, Kanada, Ungarn oder Australien, die 1990 noch höhere F\&E-Quoten als Österreich aufwiesen, konnten inzwischen überholt werden. 
Der Aufholprozess Europas gegenüber den USA verlief dagegen weniger erfreulich. Zwar wuchsen seit Mitte der 90er Jahre sowohl in den USA als auch in Europa die Ausgaben in absoluten Zahlen (siehe Abbildung 2-3, rechte Seite) und relativ zum Bruttoinlandsprodukt. Die größere Dynamik zeigten in der zweiten Hälfte der 90er Jahre aber eindeutig die USA, deren F\&E-Ausgaben sich seit 1994 mehr als doppelt so schnell entwickelten wie der Vergleichswert der Europäischen Union. Die USA sind damit auch für einen Gutteil des Anstiegs in der OECD in diesem Zeitraum verantwortlich. Der Abstand zwischen den USA und Europa hat sich deutlich vergrößert, und zwar sowohl in absoluten Größen als auch bei den relativen Ausgaben in \% des BIP. Hingegen hat sich der Österreichs zu Abstand den USA während der 90er Jahre deutlich verringert.

Abbildung 2-3: Globale Trends bei den Ausgaben für Forschung und Entwicklung, 1981-1999

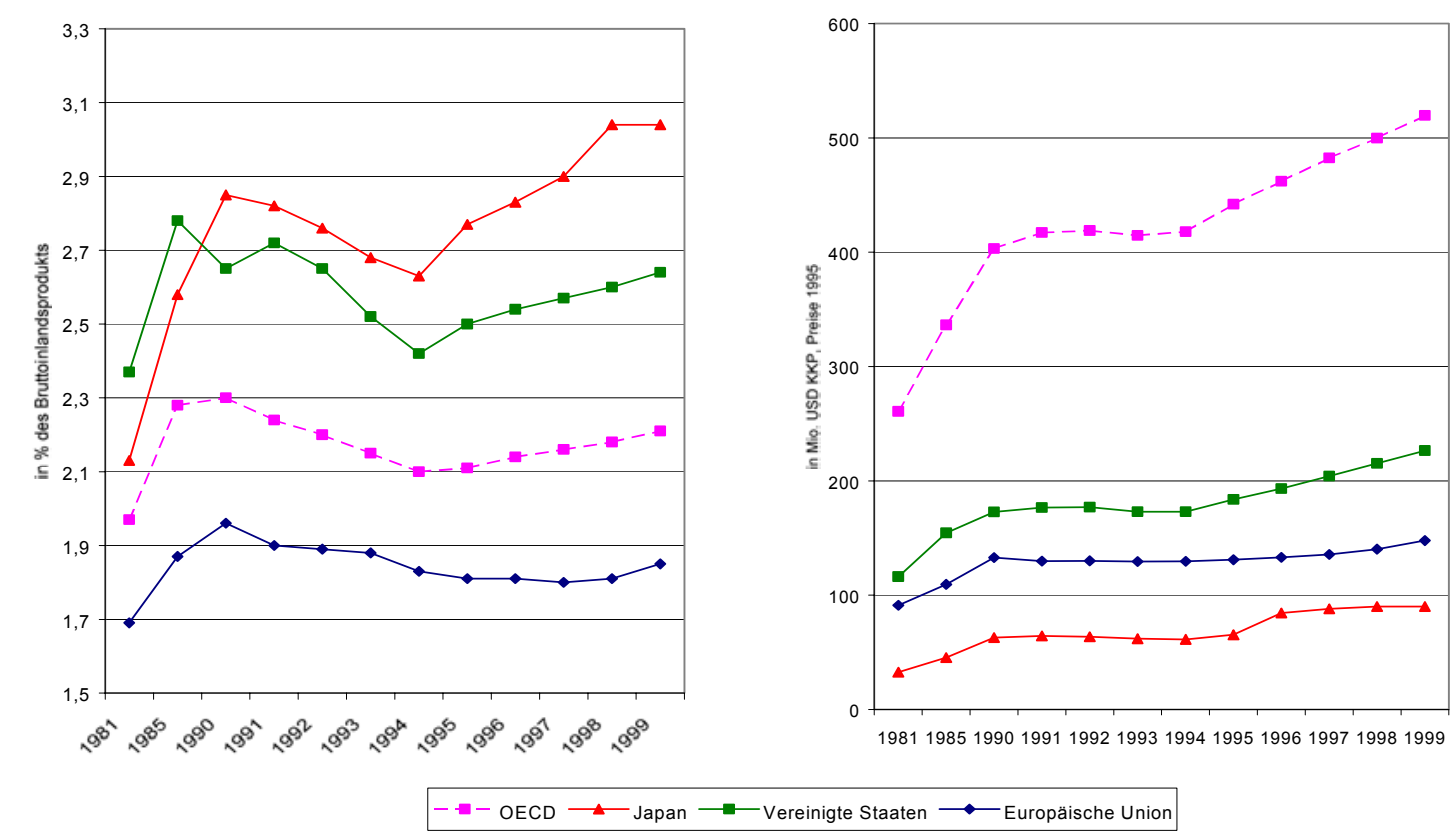

Quelle: OECD 2001

Niveau und Dynamik der Entwicklung der F\&E-Quoten werden in der folgenden Abbildung 2-4 gemeinsam betrachtet. Dabei sind die Wachstumsraten des GERD auf der horizontalen und die dazugehörige F\&E-Quote auf der vertikalen Achse abgetragen. Beide Werte sind auf den OECD-Durchschnitt normiert, sodass ein Land, dass bei einem der beiden Indikatoren überdurchschnittlich zum OECD-Durchschnitt abschneidet, sich im positiven Bereich der Achse befindet. Aus der Kombination des Wachstums- und Niveauindikators lassen sich die Länder in vier Gruppen einteilen, und zwar in solche die: 
- ihren Vorsprung ausbauen (F\&E-Quote und Wachstum des GERD überdurchschnittlich)

- aufholen (unterdurchschnittliche F\&E-Quote, überdurchschnittliches Wachstum)

- auf hohem Niveau stagnieren (überdurchschnittliche F\&E-Quote, jedoch unterdurchschnittliches Wachstum)

- weiter zurückfallen (F\&E-Quote und Wachstum unterdurchschnittlich)

Zu den Ländern, die weit überdurchschnittliche F\&E-Quoten haben und durch überdurchschnittliche Wachstumsraten ihren Vorsprung weiter ausbauen, gehören die USA, Korea und Island, aber auch die bekannten europäischen Paradeländer Schweden und Finnland.

\section{Abbildung 2-4: Niveau und Dynamik der Ausgaben für Forschung und Entwicklung verschiedener Länder, Abweichung vom OECD-Durchschnitt}

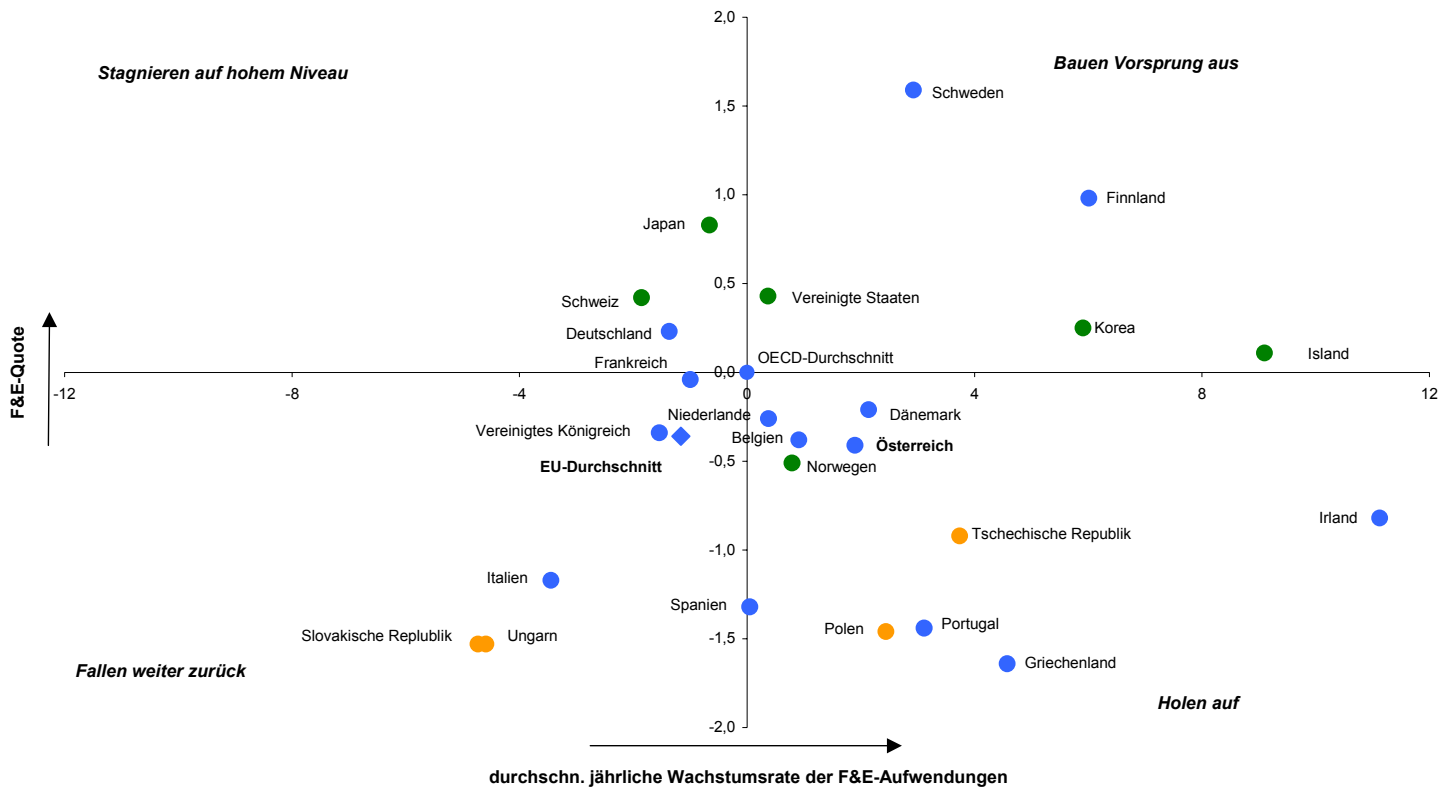

F\&E-Quoten für 1999, mit Ausnahme von Schweiz (1996), Irland, Griechenland, Tschechische Republik (1997), Niederlande (1998)

Wachstumsraten im Zeitraum 1991-1999, mit Ausnahme von Irland (bis 1997), Griechenland (bis 1997), Tschechische Republik (bis 1997), Portugal (1992-1999), Polen (1994-1999), Belgien (1993-1997), Spanien (1992-1999), Niederlande (1991-1998), Japan (1996-1999), Deutschland (1992-1999) Schweiz (1992-96), Ungarn (1994-99), Slowakische Republik (1994-99)

Quelle: OECD 2001, eigene Berechnungen

Österreich liegt in der Gruppe der aufholenden Länder, zu der auch Belgien, Dänemark, die Niederlande und Norwegen gehören. Gemeinsam mit diesen genannten Staaten ist Österreich dem OECD-Schnitt bereits wesentlich näher als etwa Griechenland, Irland oder die Tschechische Republik. Andererseits zeigen sie aber auch weniger Dynamik als diese Länder. Schlusslichter, was Niveau und/oder Dynamik betrifft, sind die südeuropäischen 
Mitgliedsstaaten der EU sowie die mittel- und osteuropäischen Beitrittskandidaten (orange), wobei Polen und die Tschechische Republik, die mit Abstand dynamischste Entwicklung aufweisen, während Italien, Ungarn und die Slowakische Republik weiter zurückfallen. Japan, die Schweiz und Deutschland schließlich stagnieren auf hohem Niveau.

Deutlich zu erkennen ist weiters, dass es nur wenig Konvergenz zwischen den EU-Staaten (blau gekennzeichnet) gibt. Die Mitgliedsländer der Union sind in allen vier Quadranten zu finden, wobei die italienische F\&E-Quote sogar zurückging. Die Abbildung zeigt auch anschaulich den Hauptgrund für den größer werdenden Abstand der EU-Staaten zu den USA. Es ist vor allem das langsamere Wachstum der F\&E-Quoten großer EU-Länder wie Deutschland, Italien, Frankreich und Großbritannien. Diese Länder waren aufgrund ihrer Größe dafür verantwortlich, dass sich während des letzten Jahrzehnts der Abstand Europas zu den USA trotz der dynamischen Entwicklung in einigen kleineren EU-Staaten wie Finnland, Schweden und auch Österreich ausgeweitet hat.

\subsection{Sektorale Struktur der Finanzierung und Durchführung von Forschung und Entwicklung}

Ein zweiter wichtiger Indikator zur Charakterisierung nationaler Innovationssysteme ist der Anteil einzelner Sektoren an der Finanzierung und Durchführung von Forschung und Entwicklung. Die Einflussfaktoren, die auf die Arbeitsteilung innerhalb des Nationalen Innovationssystems wirken, sind mannigfaltig. Üblicherweise sind Unternehmen sowohl die wichtigste Finanzierungsquelle als auch die größten Ausführenden von F\&E. Daneben finanziert der Staat F\&E direkt und indirekt durch verschiedene Förderungen und betreibt selbst Forschung an Universitäten und staatlichen Einrichtungen.

Wie die Abbildung 2-5 zeigt, bestehen auch bei diesem Indikator beträchtliche Unterschiede zwischen den verschiedenen europäischen Ländern sowie den USA und Europa. Beispielsweise haben staatliche Einrichtungen und Hochschulen für die Finanzierung von Forschung und Entwicklung in Europa einen wesentlich höheren Stellenwert als in den USA oder Japan. Analog dazu ist die Bedeutung der Unternehmen geringer. Der Staatsanteil bei Finanzierung und Durchführung ist während der letzten 10 Jahre in der Mehrheit der OECDStaaten gesunken. So finanziert der Unternehmenssektor in Japan über $70 \%$ und in den USA $67 \%$ aller F\&E-Ausgaben. In der Europäischen Union beträgt dieser Anteil lediglich 55\%. Die Spanne der Werte innerhalb der Union reicht dabei vom Schlusslicht Portugal (21\%) bis zu $69 \%$, die Belgien und Irland erreichen. Ausnahmen in Europa sind auch hier einige nordische Länder, die ähnlich hohe Unternehmensanteile wie die USA aufweisen. Ähnliches gilt für die Durchführung von Forschung und Entwicklung. Der Unternehmenssektor tätigt auch hier in den meisten Ländern mehr als die Hälfte aller Aktivitäten, wobei sein Anteil in den USA besonders hoch ist.

Die höheren Anteile des Unternehmenssektors bei Finanzierung und Durchführung von F\&E sind einer der Gründe für den sich vergrößernden Vorsprungs der USA auf Europa in den F\&EAusgaben. Einerseits wuchsen während der 90er Jahre in der Triade die F\&E-Ausgaben des öffentlichen Sektors wesentlich langsamer als jene des Unternehmenssektors. Folglich erlebten 
Länder mit größeren Unternehmensanteilen an der Finanzierung von F\&E auch überdurchschnittliche Wachstumsraten der gesamtwirtschaftlichen F\&E-Aufwendungen. Andererseits wuchsen die F\&E-Ausgaben des US-Unternehmenssektors selbst schneller als jene des EUUnternehmenssektors. Nach Angaben der OECD steigerten sich zwischen 1994 und 1999 die Ausgaben des US-Unternehmenssektors für F\&E um 40\%, während Europa nur einen Wert von $18 \%$ erreichte. Die USA verdanken also ihren wachsenden Vorsprung einem Unternehmenssektor, dessen F\&E-Investitionen schneller steigen als jene der europäischen Konkurrenten und der außerdem größeren Anteil an den gesamten F\&E-Aufwendungen hat.

\footnotetext{
Charakteristisch für das österreichische Innovationssystem ist der im internationalen Vergleich niedrige Finanzierungsanteil des Unternehmenssektors. Dem steht ein international hoher Anteil der Finanzierung durch das Ausland gegenüber, die überwiegend an den Unternehmenssektor fließt. Hervorzuheben ist auch ein relativ hoher Anteil des Hochschulsektors an den Forschungsausgaben.
}

Ein relativ geringer Anteil des Unternehmenssektors (heimische Unternehmen), aber hoher Staatsanteil ist auch für das österreichische Innovationssystem charakteristisch, wobei der Sektor Staat auch Landes- Gemeinde- und Kammerinstitutionen sowie die SV-Träger umfasst. Seine Finanzierungsstruktur entspricht damit jener von Ländern wie Italien, Ungarn oder Polen, die jedoch eine deutlich niedrigere Forschungsquote aufweisen. Wir sehen, dass der österreichische Unternehmensanteil an der gesamten F\&E-Finanzierung in Österreich etwa 10 Prozentpunkte unter dem von Ländern wie Dänemark oder den Niederlanden liegt, die in Niveau und Dynamik mit Österreich vergleichbar sind. Die größten Abweichungen von der Trendgeraden finden sich in Transformationsökonomien und den beiden EU-Mitgliedsstaaten Griechenland und Portugal. Während der 90er Jahre wurde die Dynamik der F\&EAufwendungen eindeutig von den Unternehmen getragen. Die hohen Steigerungsraten der F\&E-Ausgaben (GERD), die Länder wie Schweden oder Finnland aufwiesen, sind größtenteils auf die höheren F\&E-Investitionen von Unternehmen wie Nokia, Ericsson, Saab oder Pharmafirmen zurückzuführen. Dadurch ist zumindest ein Teil des Zusammenhangs zwischen Struktur und Höhe der F\&E-Ausgaben erklärbar.

Als besonderes Charakteristikum weist Österreich jedoch einen hohen Finanzierungsbeitrag aus ausländischen Quellen auf, der im Forschungs- und Technologiebericht 2001 bereits im Detail beschrieben wurde. Mit einem Anteil von etwa 20\% erreicht Österreich einen Spitzenwert unter den OECD-Ländern. Die F\&E-Erhebung 1998 von Statistik Austria gibt Aufschluss über die Verflechtung von Finanzierungs- und Durchführungssektoren (siehe Tabelle 29 im statistischen Anhang). Dabei zeigt sich, dass die aus dem Ausland finanzierten F\&E-Aktivitäten überwiegend (zu 95\%) an den heimischen Unternehmenssektor fließen. Nur ein relativ geringer Teil dieser Mittel stammt aus den Forschungsprogrammen der EU, der weitaus größte Teil wird von Unternehmen im Ausland finanziert. Der relativ geringe Anteil des Unternehmenssektors (Unternehmen im Inland) an der Finanzierung der F\&E-Aktivitäten in Österreich wird dadurch insofern relativiert als ihm ein hoher Finanzierungsanteil von Unternehmen im Ausland 
gegenübersteht. Dies weist darauf hin, dass sich Österreich als attraktiver Forschungsstandort, insbesondere für Forschung in Unternehmen, etablieren konnte.

Auf der Durchführungsseite fällt an den österreichischen Daten zum einen der relativ hohe Anteil an Hochschulforschung auf, der mit beinahe 30\% um 10 Prozentpunkte höher als der EUDurchschnitt ist. Laut F\&E-Erhebung 1998 wurde die im Hochschulsektor durchgeführte F\&E nahezu ausschließlich durch den öffentlichen Sektor finanziert (siehe Tabelle 29 im statistischen Anhang). Der Finanzierungsanteil des Unternehmenssektors (ein wichtiger Indikator der Verflechtung zwischen Universität und Wirtschaft) betrug lediglich 1,7\% und war damit gleich hoch wie die Finanzierung durch die Europäische Union. Gleichzeitig ist das Ausmaß, in dem der Staat selbst Forschung durchführt, in Österreich wesentlich geringer als in der Europäischen Union oder in den mittel- und osteuropäischen Nachbarländern. Allerdings werden in Österreich die Einrichtungen des außeruniversitären kooperativen Forschungsbereichs wie etwa die Austrian Research Centers, die als Kapitalgesellschaften organisiert sind, zum Unternehmenssektor gezählt. Gemeinsam trägt dieser "kooperative Bereich" etwa 5\% zur gesamten heimischen Forschungsleistung bei. Charakteristisch für das österreichische Innovationssystem ist weiters das Fehlen privater Non-profit-Organisationen, die im OECDSchnitt $2,5 \%$ der Forschungsleistung erbringen.

\section{Abbildung 2-5: Anteile verschiedener Sektoren an der Finanzierung und Durchführung von Forschung und Entwicklung, 1999}
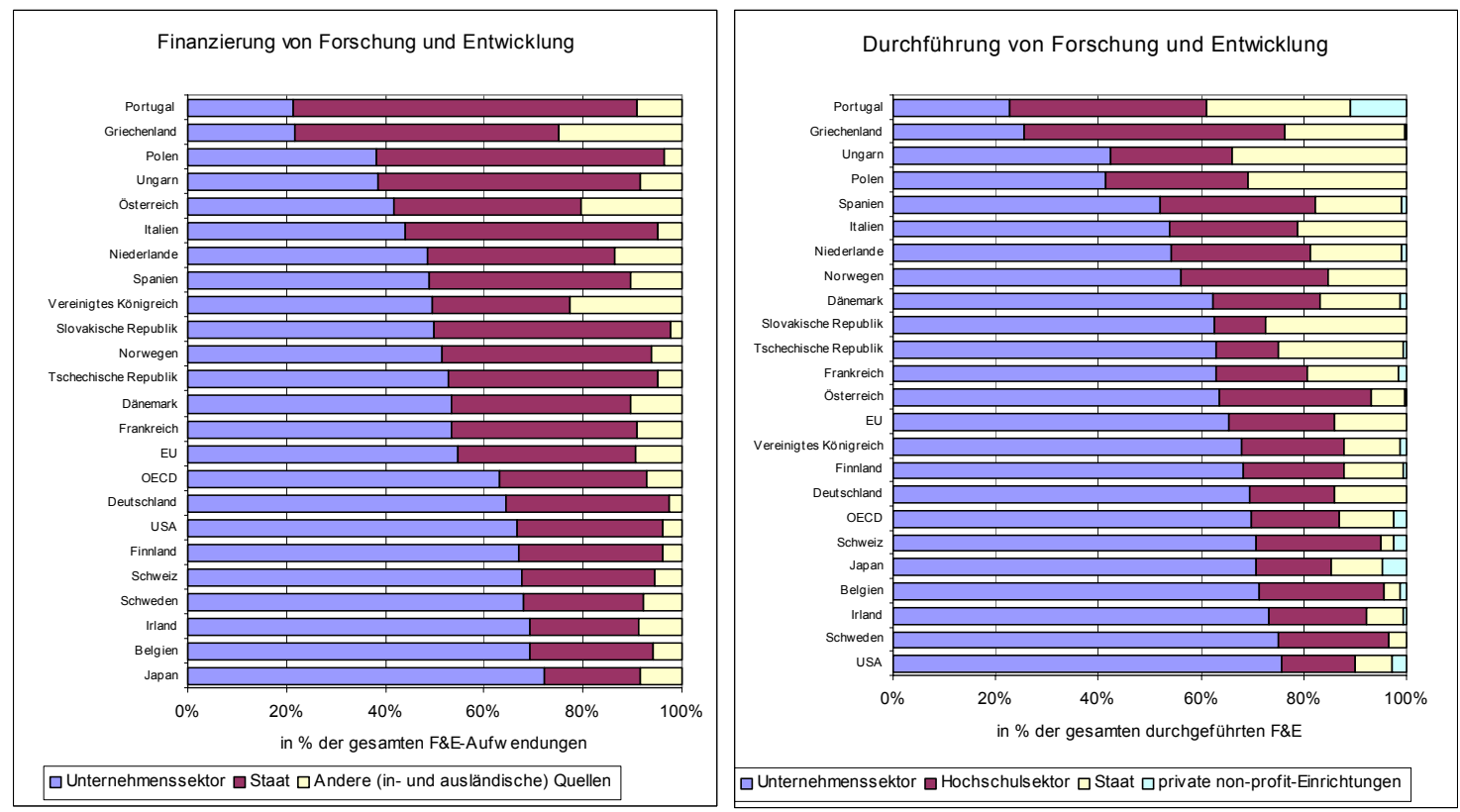

Werte für 1999, mit Ausnahme von der Schweiz (1996), Irland, Griechenland, Tschechische Republik (1997), Niederlande (1998)

Quelle: OECD 2001

Der F\&E-Erhebung 1998 ist überdies eine weitere wichtige Kennzahl, der Finanzierungsanteil des öffentlichen Sektors an der im Unternehmenssektor durchgeführten F\&E (also eine 
Maßzahl der direkten F\&E-Förderung der öffentlichen Hand) zu entnehmen. Dieser Anteil betrug 1998 5,5\% und liegt damit signifikant niedriger als in der letzten F\&E-Erhebung (1993).

Der relativ geringe Anteil der Unternehmen bei Finanzierung und Durchführung von Forschung in Österreich - auch wenn bedeutende Finanzierungsanteile von verbundenen Unternehmen aus dem Ausland geleistet werden - ist die zentrale Strukturschwäche des österreichischen Innovationssystems, denn gerade die Unternehmen haben zum Wachstum der F\&E-Ausgaben während der 90er Jahre überproportional beigetragen. Die Bemühungen der österreichischen Bundesregierung, die Forschungsquote auf 2,5\% des BIP zu heben, müssen deshalb auch bei der Finanzierungsstruktur ansetzen und sie vor allem durch die Stimulierung der Unternehmensforschung verändern. Diese Notwendigkeit ergibt sich schon aus der Konsolidierung der öffentlichen Haushalte, die Zuwächse bei Forschung und Entwicklung im öffentlichen Sektor auch in Zukunft relativ eng begrenzen wird. 


\section{Grundlagenforschung und Wissenschaftssystem}

\subsection{Internationale Trends in der Grundlagenforschung}

Unter Grundlagenforschung wird experimentelle oder theoretische wissenschaftliche Arbeit verstanden, die primär neues Wissen erschließen oder bekannte Phänomene erklären soll, jedoch nicht eine bestimme Anwendung als Ziel hat (vgl. OECD 1994). Dieser Art von Forschung wird im Allgemeinen der Charakter eines öffentlichen Gutes zugeschrieben. Da Märkte für die Produktion von öffentlichen Gütern nur unzureichende Anreize bieten können, haben sich für Grundlagenforschung andere, nicht-marktmäßige Anreizsysteme herausgebildet und der öffentliche Sektor oder gemeinnützige Einrichtungen vielfach ihre Finanzierung übernommen (vgl. Stephan 1996).

Grundlagenforschung ist nicht auf die Entwicklung einer bestimmten Anwendung gerichtet. Zwischen der Erforschung verschiedener Phänomene und ihrer wirtschaftlichen Nutzung liegen jedoch meistens lange zeitliche Verzögerungen. Trotzdem ist Grundlagenforschung für unternehmerische Innovation und wirtschaftliche Entwicklung unverzichtbar (siehe Box). Darüber hinaus ist ein wichtiger Grund für die Förderung von Grundlagenforschung die Unsicherheit des technologischen Forschritts selbst: Wir können heute nur erahnen, auf welchen wissenschaftlichen Grundlagen die Basisinnovationen des Jahres 2020 aufbauen werden. Daher ist öffentlich finanzierte und thematisch ungebundene Grundlagenforschung notwendig, um möglichst breite wissenschaftliche Basis zu sichern (vgl. Callon 1994). So war die Prionenforschung bis zum Auftreten von BSE eine Disziplin mit sehr geringem Anwendungsbezug.

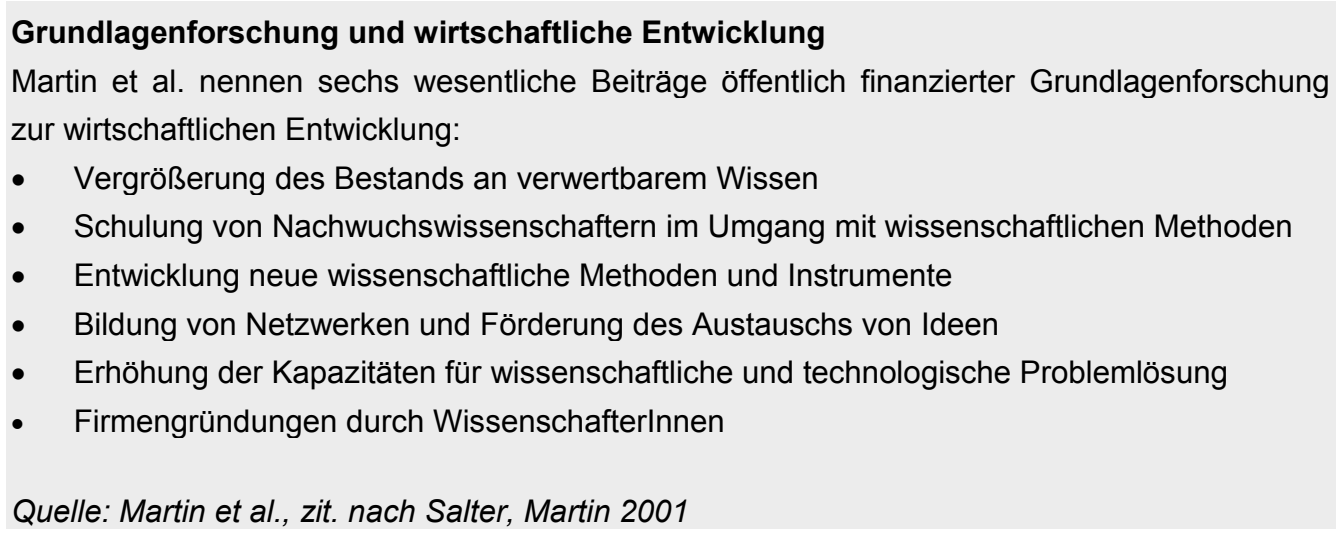

Seit Mitte der 90er Jahre lässt sich im gesamten OECD-Raum eine Steigerung der F\&EAusgaben beobachten. Diese steigende F\&E-Intensität hatte auch Änderungen im Finanzierungsmuster zur Folge: Typischerweise wurden die Zuwächse hauptsächlich vom Unternehmenssektor getragen. Der Anteil des öffentlichen Sektors ist dagegen gesunken. Trotz dieser Änderung im Muster der Forschungsfinanzierung ist der Anteil von Grundlagenforschung an den gesamten F\&E-Ausgaben nicht notwendigerweise zurückgegangen. Vielmehr zeigen die 
(spärlich) vorhandenen Daten, dass die Grundlagenforschung in den einzelnen Ländern von diesen Entwicklungen sehr unterschiedlich betroffen war. Für die OECD-Länder ist im Durchschnitt der Anteil der Grundlagenforschung an den gesamten F\&E-Ausgaben von 19,4\% Anfang der 80er Jahre auf 21,2\% Ende der 90er Jahre gestiegen. Die folgende Abbildung 3-1 zeigt die Veränderungen der Ausgaben für Grundlagenforschung in Prozent der gesamten Forschungsausgaben für einzelne ausgewählte Länder zwischen dem Beginn der 80er Jahre und dem Ende der 90er Jahre.

\section{Abbildung 3-1: Entwicklung der Grundlagenforschung in \% der gesamten F\&E-Ausgaben}

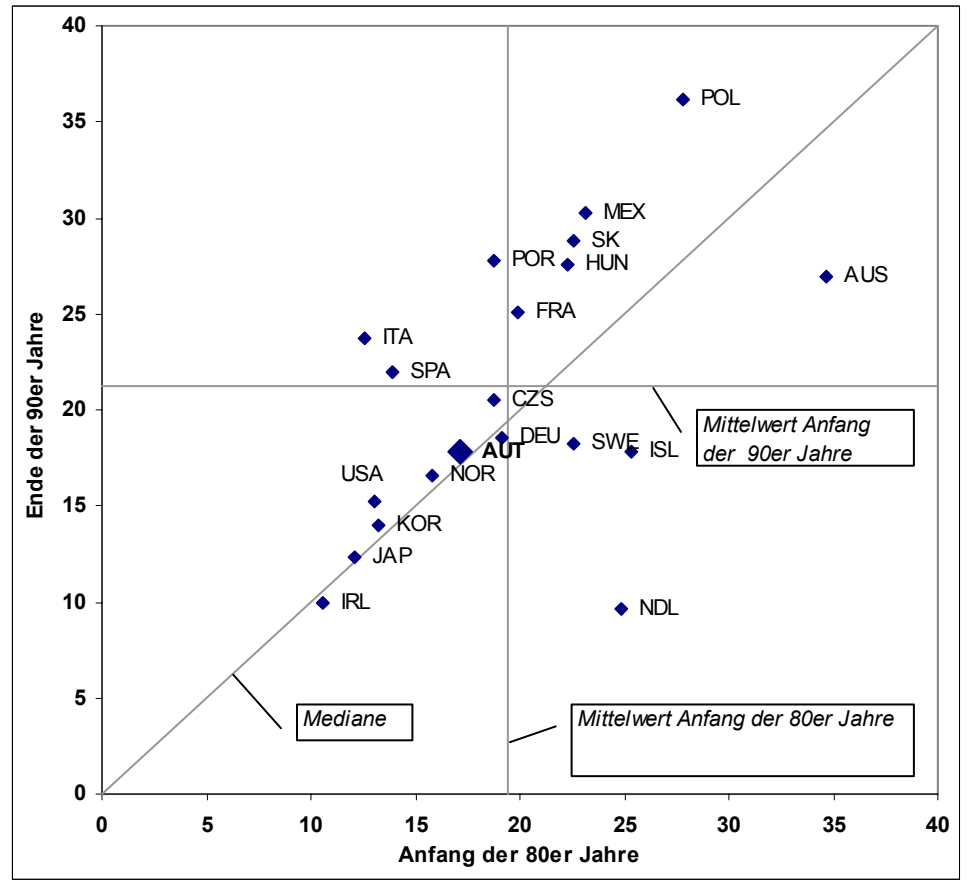

Quelle: OECD 2001

Länder, die auf der $45^{\circ}$-Linie (1. Mediane) liegen, haben keine Veränderungen ihrer relativen Ausgaben für Grundlagenforschung in diesem Zeitraum erfahren. Dazu zählen Irland, Japan, Norwegen, Deutschland und Österreich. Länder, die über der $45^{\circ}$-Linie zu liegen kommen, konnten hingegen den relativen Anteil der Grundlagenforschung ausbauen. Dies sind unter anderem Frankreich, Spanien oder Italien, die damit über dem OECD-Durchschnitt liegen. In Schweden und den Niederlanden lässt sich hingegen eine deutlicher Rückgang des relativen Anteils beobachten. In Österreich betrug der Anteil der Grundlagenforschung an den gesamten F\&E-Ausgaben Anfang der 80er Jahre 17,1\%. Dieser Anteil konnte im Laufe der 90er Jahre auf über 22\% gesteigert werden. Laut F\&E-Vollerhebung 1998 (vgl. Statistik Austria 2002) betrug der relative Anteil im Jahr 1998 17,8\%. Österreich lag somit in den 80er Jahren sowie auch Ende der 90er Jahre knapp unter dem OECD-Durchschnitt.

Wie sich zeigt, lassen sich auf den ersten Blick für den gesamten OECD Raum (soweit Daten vorhanden sind) kaum allgemeine Trends in den relativen Anteilen in der Grundlagenforschung 
nach durchführenden Sektoren ablesen. Bezüglich der Rolle des Unternehmenssektors kann jedoch für einige Länder eine Steigerung des relativen Anteils in den 90er Jahren beobachtet werden. Der Unternehmenssektor in Deutschland, Spanien, den USA und Irland wies Anfang der 80er Jahre den gleichen relativen Anteil an der Grundlagenforschung auf wie Österreich (ca. 18\%). Deren Anteil konnte sich in den 90er Jahren deutlich erhöhen. In Korea, Japan und Irland werden jeweils ein Drittel der Grundlagenforschung vom Unternehmenssektor durchgeführt.

\section{Abbildung 3-2: Ausgaben für Grundlagenforschung nach ausführenden Sektoren in \%}
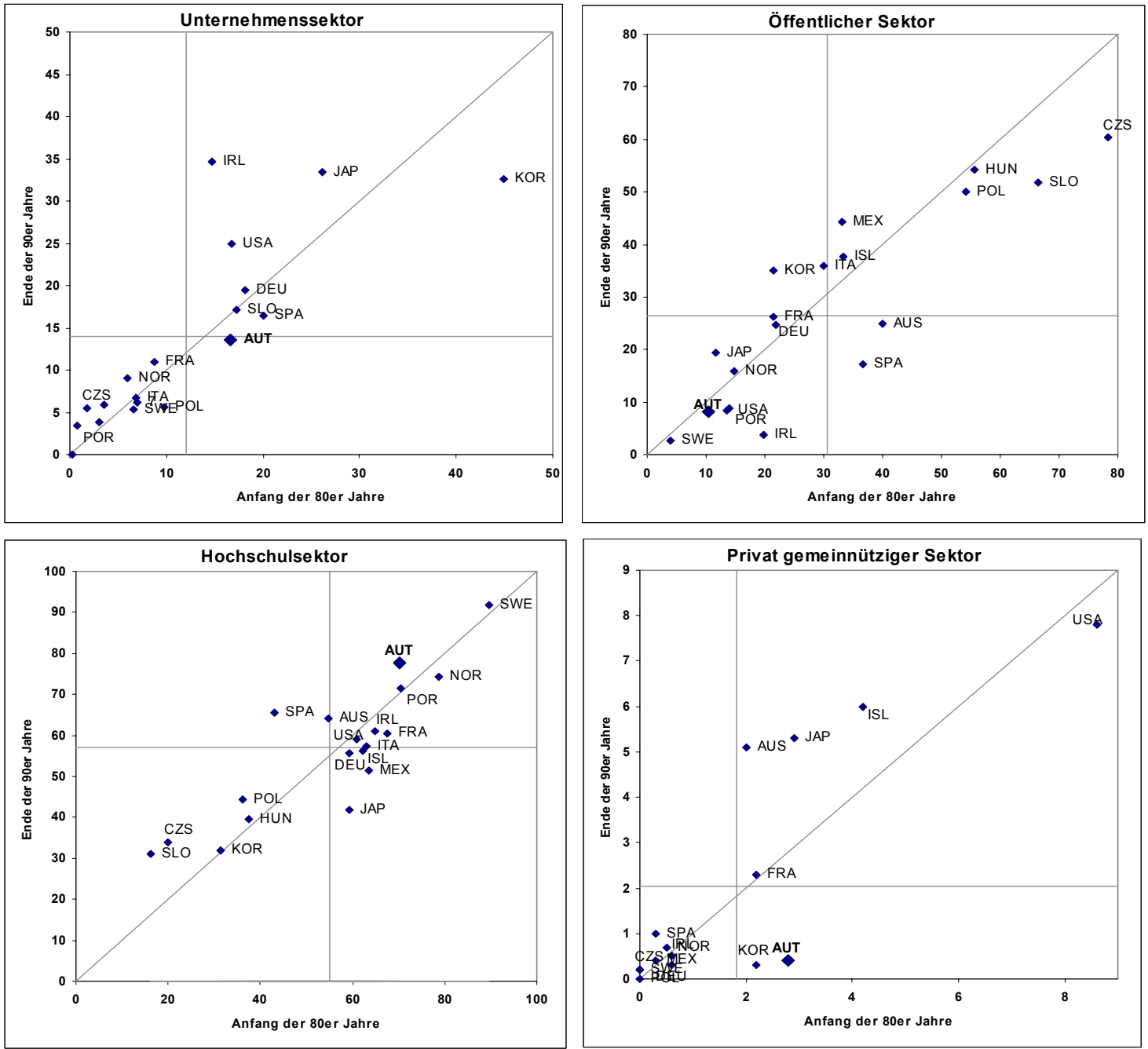

Quelle: OECD 2001

Bei der Organisation der Grundlagenforschung werden in OECD-Ländern sehr unterschiedliche Modelle verfolgt. Dies zeigt sich an der Verteilung der Ausgaben für Grundlagenforschung nach ausführenden Sektoren. In Österreich sind diese Aktivitäten vor allem im Hochschulsektor gebündelt. Dessen Anteil lag mit 70\% schon in den 80er Jahren über dem OECD Durchschnitt. In den 90er Jahren erhöhte sich der Anteil des Hochschulsektors in Österreich auf über $77 \%$. 
Verglichen mit anderen EU-Ländern weist der österreichische Unternehmenssektor sowohl Anfang der 80er Jahre als auch Ende der 90er Jahre einen erstaunlich hohen Anteil an der Grundlagenforschung auf. Relativ wenig Grundlagenforschung wird jedoch im öffentlichen und im privaten gemeinnützigen Sektor betrieben.

Eine andere Konzeption findet sich in den meisten zentral- und osteuropäischen Ländern. Hier wird der höchste Anteil der Grundlagenforschung im öffentlich-staatlichen Sektor und nur zu einem relativ geringen Teil im Hochschulsektor durchgeführt. Einschlägige Aktivitäten sind in diesen Ländern (noch immer) zu einem hohen Anteil in nationalen Wissenschaftsakademien konzentriert. Auch fehlt in den meisten westeuropäischen OECD-Staaten Grundlagenforschung in privaten, gemeinnützigen Einrichtungen, wie sie etwa in den USA zu finden ist. Der Anteil dieser Aktivitäten ist auch in Österreich im Beobachtungszeitraum stark zurückgegangen, wie Abbildung 3-2 zeigt.

Die OECD-Länder geben durchschnittlich $0,3 \%$ ihres BIP für Grundlagenforschung aus. Wie Abbildung 3-3 zeigt, variiert der Wert allerdings stark zwischen den Ländern und reicht von $0,1 \%$ des BIP in Mexiko bis zu über $0,7 \%$ in der Schweiz, die damit den höchsten BIP-Anteil aufweist. Die Höhe des Anteils der Grundlagenforschung ist dabei nicht direkt an die Höhe der gesamten F\&E-Aufwendungen gekoppelt.

Abbildung 3-3: F\&E-Ausgaben nach Forschungsarten und Anteil der Grundlagenforschung nach ausführenden Sektoren, 1999

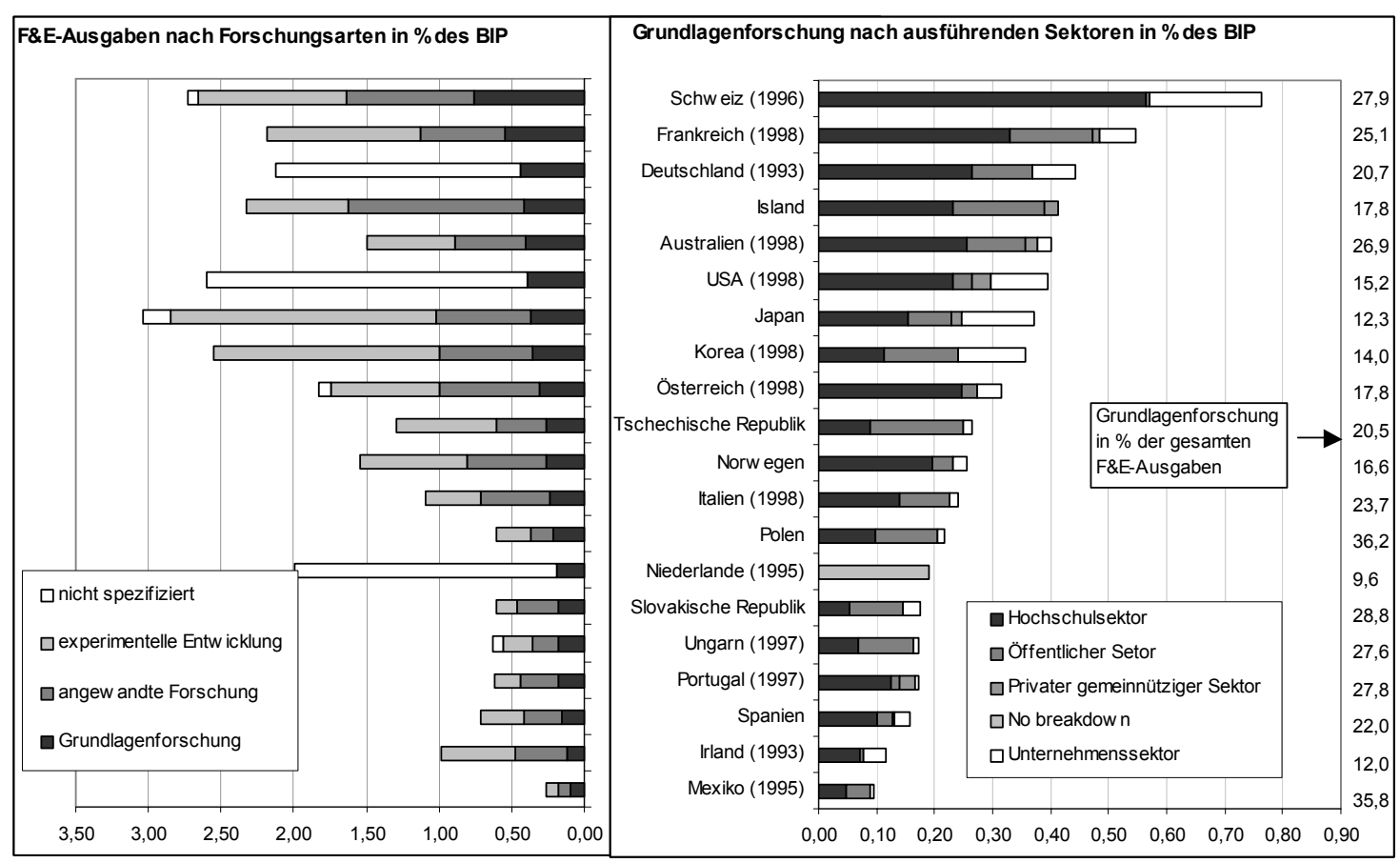

Quelle: OECD 2001

Länder wie die Slowakische Republik, Ungarn, Portugal oder auch die Tschechische Republik weisen über $20 \%$ Anteil der Grundlagenforschung an den gesamten F\&E-Ausgaben auf, 
obwohl sie wesentlich niedrigere gesamtwirtschaftliche F\&E-Quoten erreichen. Dies ist im Wesentlichen mit dem geringen Anteil des Unternehmenssektors an den gesamten F\&EAusgaben zu erklären.

\subsection{Publikationen österreichischer Wissenschafterlnnen}

Wissenschaftliche Fachzeitschriften gehören zu den wesentlichsten Medien, um Forschungsergebnisse von hohem Neuigkeitscharakter und hoher Relevanz an eine internationale wissenschaftliche Gemeinschaft kommunizieren. Das folgende Kapitel bezieht seine Analysen auf Daten des Science Citation Index. In dieser Datenbank werden Publikationen in 8.500 Fachzeitschriften aus aller Welt und die Liste ihrer Referenzen gesammelt. Die Fachzeitschriften kommen aus Technischen und Naturwissenschaften, Sozial- und Wirtschaftswissenschaften, Agrarwissenschaften, Veterinär- und Humanmedizin. In der Datenbank werden sie ausschließlich in einer von über 20 wissenschaftlichen Sub-Disziplinen klassifiziert. Jeder Autor wird gemäß seiner angegebenen Adresse einem Land zugeordnet. Die Zusammenfassung der Anzahl der Publikationen auf Länderebene erfolgt über die Autoren und ihre Länderzugehörigkeit: Eine Publikation wird mehreren Ländern zugerechnet, wenn Autoren mit Adressen aus mehreren Ländern daran beteiligt waren.

\section{Abbildung 3-4: Publikationen in wissenschaftlichen Fachzeitschriften im} Ländervergleich, 1996-2000

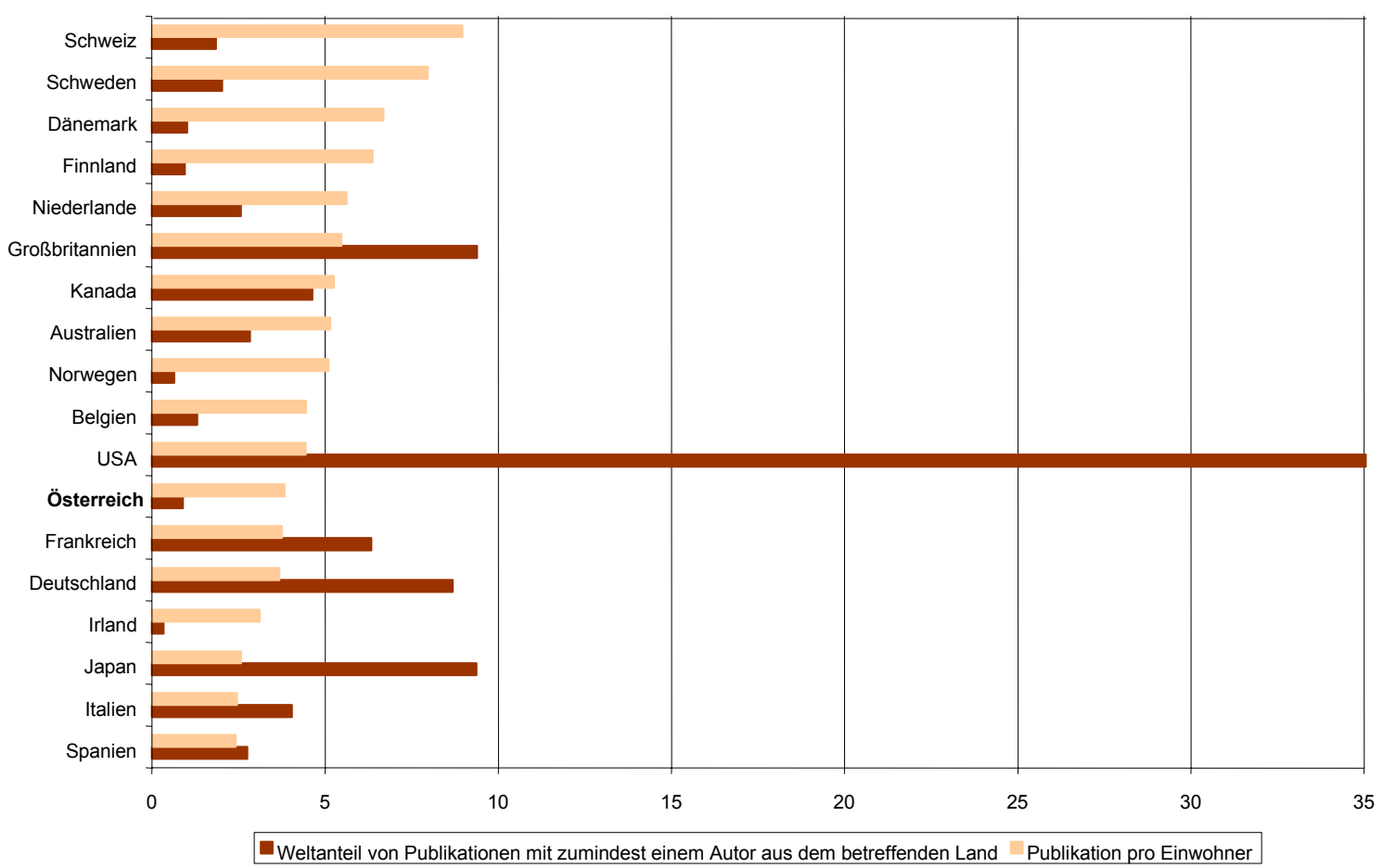

Quelle: Science Citation Index, OECD, eigene Berechnungen 
Abbildung 3-4 zeigt den Weltanteil von Publikationen mit zumindest einem Autor aus dem betreffenden Land im Ländervergleich. Allen voran liegen die USA: Etwa 35 Prozent aller weltweiten Publikationen in wissenschaftlichen Fachzeitschriften haben zumindest einen Autor aus den USA. Mit einigem Abstand folgen Großbritannien und Deutschland mit jeweils etwa 9 Prozent der weltweiten Publikationen.

Betrachtet man die Anzahl der Publikationen eines Landes normiert auf die Größe des betreffenden Landes, zeigt sich naturgemäß ein völlig anderes Bild: Die USA liegen nun im Mittelfeld, gefolgt von Österreich. An der Spitze der Publikationen pro Einwohner liegen nun kleine Länder wie die Schweiz, Schweden und Dänemark. Österreich liegt nur im europäischen Mittelfeld.

Abbildung 3-5 bildet das thematische Muster österreichischer Forschung im Vergleich zum Rest der Welt ab. Unter allen Fachrichtungen haben österreichische WissenschafterInnen den relativ größten Anteil an weltweiten Publikationen auf dem Gebiet der Immunologie. Er beträgt 1,32 Prozent aller Publikationen in diesem Fach. Es folgen Klinische Medizin mit 1,27 Prozent und Physik mit 0,94 Prozent der weltweiten Publikationen in diesen Gebieten. Anteilsmäßig am geringsten fallen die Publikationen österreichischer Wissenschaftler in Fachzeitschriften der Sozial- und Wirtschaftswissenschaften, der Agrarwissenschaften und Psychologie/Psychiatrie aus.

\section{Abbildung 3-5: Anteil der weltweiten Publikationen mit zumindest einem Autor aus} Österreich, 1996-2000

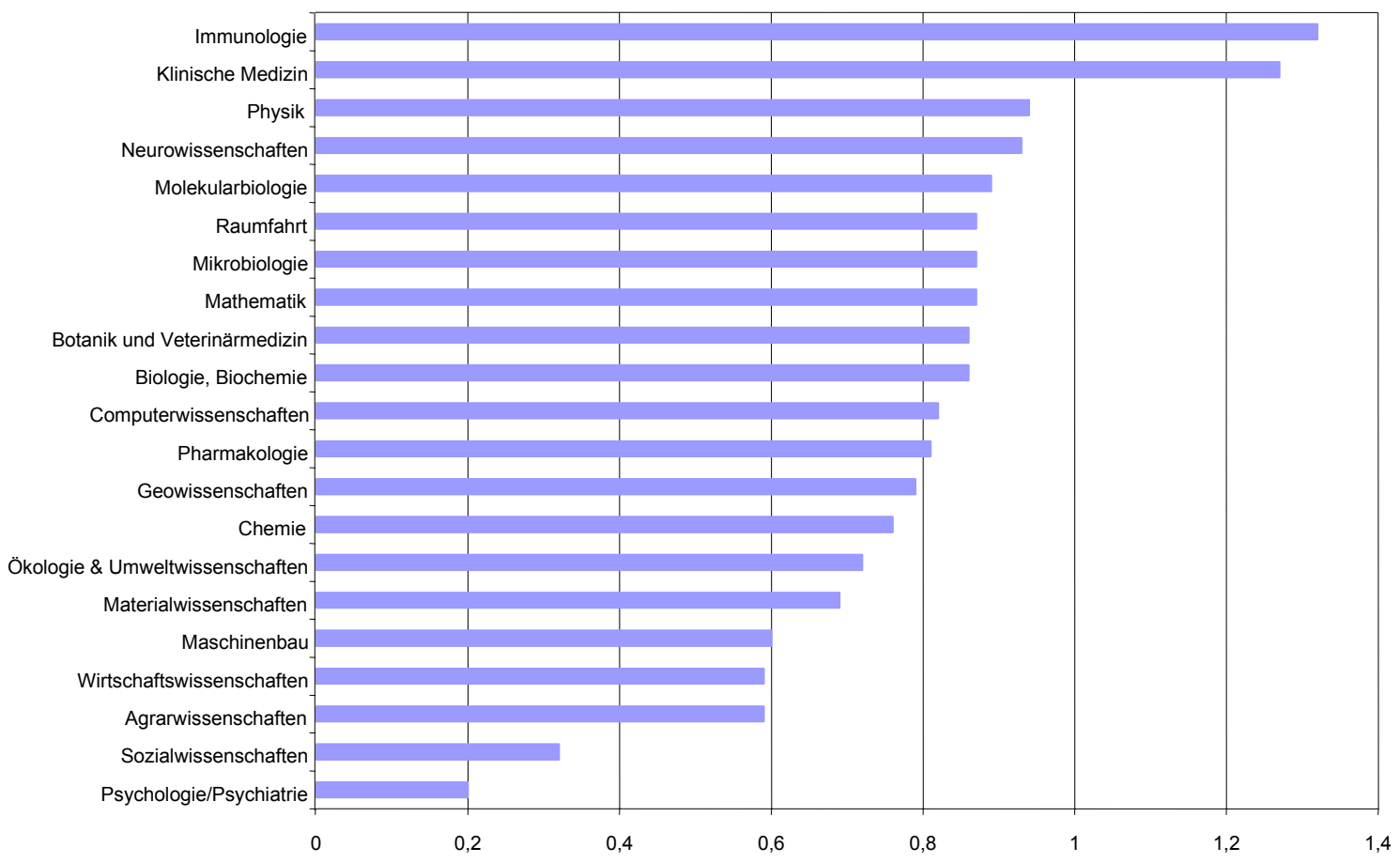

Quelle: Science Citation Index. Angaben in Prozent. 
Neben dem quantitativen Anteil an den Erkenntnissen der Scientific Community lässt sich die Qualität der Forschung durch den Grad messen, in dem die Publikationen von anderen WissenschafterInnen zitiert werden. Dieser Impact Factor zeigt, in welchem Ausmaß Publikationen, an denen zumindest ein Autor aus Österreich beteiligt war, im Vergleich zum Weltdurchschnitt zitiert werden. Nimmt der Indikator einen positiven Wert an, bedeutet das eine zum Weltdurchschnitt überdurchschnittliche Zitierhäufigkeit, ein negativer Wert dagegen unterdurchschnittliche Zitierhäufigkeit.

Wie Abbildung 3-6 zeigt, werden österreichische Publikationen in der Mehrzahl der Fachgebiete weniger häufig als der Weltdurchschnitt zitiert. Die höchste Resonanz erreicht Österreich auf dem Gebiet der Physik, wo die Publikationen aus Österreich um 26 Prozent häufiger zitiert werden als der Weltdurchschnitt, in Mathematik 20 Prozent häufiger und in Pharmakologie 15 Prozent häufiger. Publikationen aus Mikrobiologie mit mindestens einem Autor aus Österreich werden exakt gleich häufig wie der Weltdurchschnitt zitiert. In der überwiegenden Zahl der Fachgebiete weist Österreich allerdings negative Impact-Faktoren auf.

\section{Abbildung 3-6: Der Impact-Factor von Publikationen mit zumindest einem Autor aus Österreich, 1996-2000}

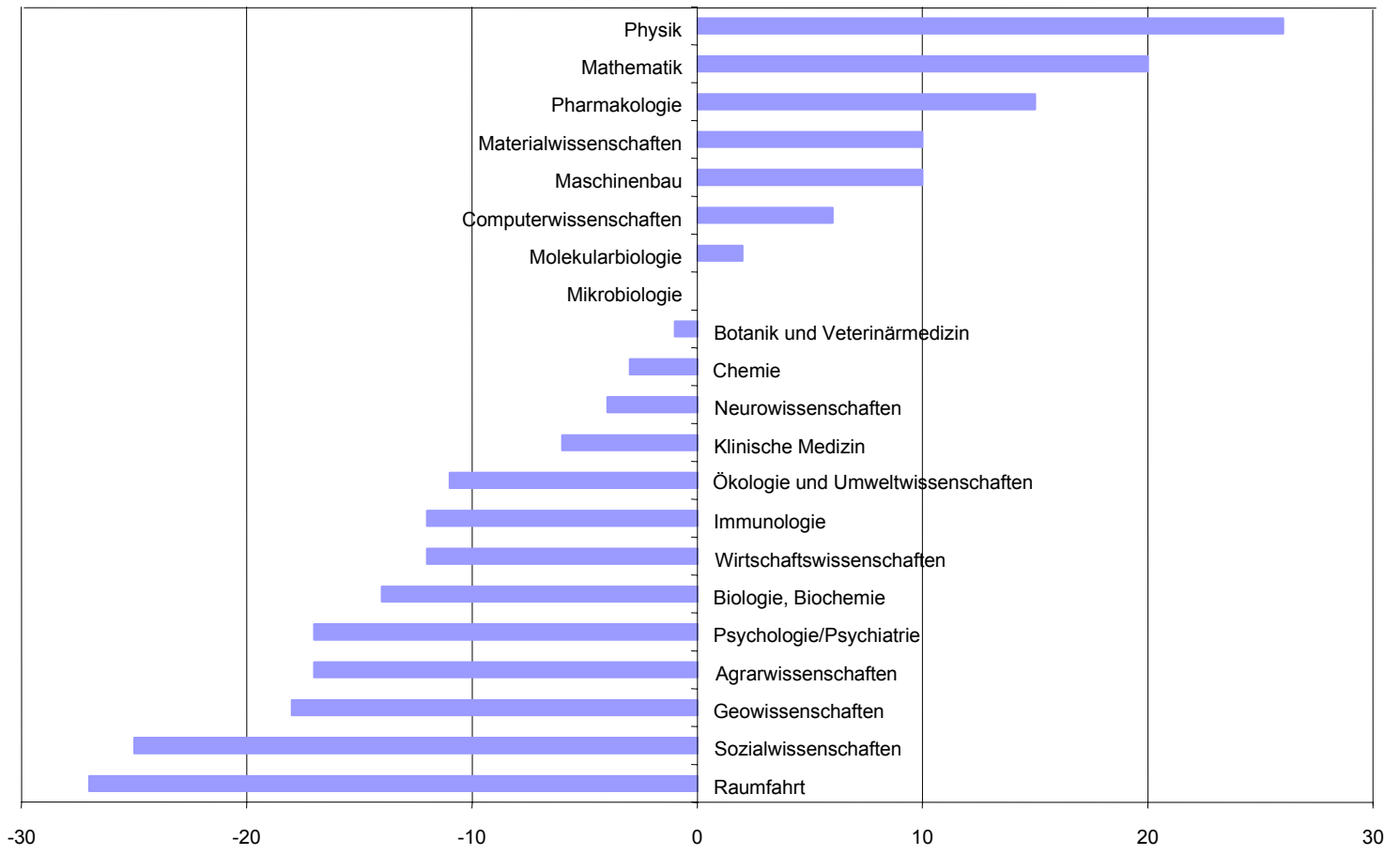

Quelle: Science Citation Index. Prozentuelle Abweichung vom Weltdurchschnitt.

Allgemein gilt, dass geringe Impact-Faktoren auf zweierlei hindeuten können: (i) die geringe Resonanz einer Publikation, bzw. der Publikationen einer Sub-Disziplin in der scientific community aufgrund eines geringen Neuigkeitsgehaltes oder geringer Relevanz der Publikationen, oder (ii) aufgrund eines hohen Spezialisierungsgrades der Publikationen. Ein Vergleich aus Abbildung 3-5 mit Abbildung 3-6 etwa macht deutlich, dass Österreich auf den 
Gebieten der Immunologie und der Klinischen Medizin einen hohen Anteil an Publikationen aufweist, die jedoch in geringerem Ausmaß als der Weltdurchschnitt zitiert werden - ihr ImpactFaktor beträgt -12 und -6 respektive. Wir schließen daraus, dass die Publikationen in diesen beiden Fachbereichen einen hohen Spezialisierungsgrad aufweisen. Die Lancierung einer Publikation in einer Fachzeitschrift stellt aufgrund der damit verbundenen Bewertung durch anerkannte Wissenschaftler aus dem Gebiet ein Qualitätskriterium an dar; wenn im Fall österreichischer Publikationen ein geringer Neuheitsgrad vorliegen würde, wäre wohl kaum ein überproportionaler Anteil am weltweiten Output möglich.

Tabelle 3-1 zeigt zu Beginn ausgewählte 14 Länder geordnet nach der Anzahl ihrer Bevölkerung im Jahr 2000. Dies dient zur Orientierung und besseren Einschätzung der unterhalb angeführten Werte: Sie bezeichnen den Anteil der weltweiten Publikationen pro SubDisziplin in einem Land. Die Sub-Disziplinen sind zusammengefasst zu Disziplinen auf der einstelligen Ebene der International Standard Classification of Education (ISCED). Eine Ausnahme bilden dabei die Naturwissenschaften, die differenzierter dargestellt sind mit Naturund Lebenswissenschaften. Die Länder sind geordnet nach ihrem durchschnittlichen Anteil an den weltweiten Publikationen pro einstelliger ISCED-Disziplin.

Wie oben erwähnt, ist die Positionierung einer Publikation in einer Fachzeitschrift aufgrund des damit verbundenen Review-Prozesses, der die Publikationen auf thematische Relevanz und methodische Qualität prüft, ein Qualitätsmerkmal an sich. Österreich konnte im Zeitraum 19962001 in allen Disziplinen weniger Publikationen in Fachzeitschriften platzieren, als aufgrund seiner Bevölkerungszahl zu erwarten wäre: Von den hier angeführten europäischen Ländern ist Österreich das neuntgrößte Land - seine Position bezogen auf die weltweiten Anteile der Publikationen auf einstelliger Disziplin variiert zwischen Rang 11 und Rang 14. Damit ist Österreich in Relation zu seiner Größe in weltweiten Fachzeitschriften unterdurchschnittlich präsent. Die Naturwissenschaften schneiden dabei noch am besten ab: In Chemie und Physik belegt Österreich von den hier angeführten Ländern jeweils Rang 10 der weltweiten Publikationen.

Länder von vergleichbarer Bevölkerungszahl, wie Schweden und die Schweiz, können sich besser behaupten als Österreich: Schweden ist von den hier angeführten Ländern das achtgrößte Land und positioniert sich bei den weltweiten Publikationen auf Ebene der einstelligen Disziplinen zwischen dem 6. und dem 8. Rang. Die Schweiz ist von den hier angeführten Ländern das zehntgrößte Land, und positioniert sich zwischen dem 7. und der 10. Rang. Auch kleine Länder wie Finnland und Dänemark weisen in Relation zu ihrer Bevölkerungszahl in vielen Disziplinen einen hohen Anteil an weltweiten Publikationen auf. 
Tabelle 3-1: Der Anteil der einzelnen Länder an den weltweiten Publikationen pro Sub-Disziplin, 1996-2001

\begin{tabular}{|c|c|c|c|c|c|c|c|c|c|c|c|c|c|c|}
\hline Land & GE & UK & FR & IT & ES & $\mathrm{NL}$ & $\mathrm{BE}$ & SE & AT & $\mathrm{CH}$ & DK & FI & NO & IR \\
\hline Bevölkerung 2000 & 82143 & 59766 & 58892 & 57189 & 39466 & 15926 & 10251 & 8872 & 8106 & 7185 & 5337 & 5181 & 4491 & 3787 \\
\hline Naturwiss. & GE & FR & UK & IT & ES & NL & $\mathrm{CH}$ & SE & BE & DK & AT & NO & FI & IR \\
\hline Chemie & 10,51 & 6,78 & 7,14 & 4,15 & 3,94 & 1,98 & 1,90 & 1,49 & 1,29 & 0,74 & 0,76 & 0,42 & 0,64 & 0,24 \\
\hline Geowissenschaften & 8,51 & 9,38 & 11,61 & 3,84 & 2,25 & 2,51 & 2,16 & 1,86 & 0,96 & 1,15 & 0,79 & 1,74 & 0,72 & 0,25 \\
\hline Mathematik & 9,96 & 12,25 & 6,84 & 5,02 & 4,18 & 1,75 & 1,25 & 1,31 & 1,26 & 0,77 & 0,87 & 0,51 & 0,63 & 0,44 \\
\hline Physik & 11,85 & 8,59 & 7,09 & 5,02 & 2,82 & 1,88 & 2,52 & 1,63 & 1,24 & 0,92 & 0,94 & 0,35 & 0,69 & 0,24 \\
\hline Lebenswiss. & UK & GE & FR & IT & $\mathbf{N L}$ & ES & SE & $\mathrm{CH}$ & $\mathrm{BE}$ & DK & $\mathrm{FI}$ & AT & NO & IR \\
\hline Biologie und Biochemie & 9,99 & 8,07 & 6,87 & 4,11 & 2,61 & 2,74 & 2,69 & 2,01 & 1,42 & 1,59 & 1,04 & 0,86 & 0,57 & 0,38 \\
\hline Ökologie/Umweltwiss. & 9,52 & 6,26 & 4,13 & 2,63 & 3,26 & 3,19 & 3,12 & 1,74 & 1,22 & 1,72 & 1,66 & 0,72 & 1,41 & 0,24 \\
\hline Mikrobiologie & 11,24 & 9,76 & 8,09 & 3,33 & 3,85 & 4,14 & 2,12 & 2,26 & 1,96 & 1,62 & 0,94 & 0,87 & 0,63 & 0,55 \\
\hline Molekularbiologie & 10,96 & 9,88 & 7,99 & 4,26 & 2,93 & 2,45 & 2,03 & 2,65 & 1,51 & 1,08 & 1,01 & 0,89 & 0,59 & 0,23 \\
\hline Technische Wiss. & UK & GE & FR & IT & ES & NL & SE & $\mathrm{CH}$ & $\mathrm{BE}$ & FI & DK & AT & NO & IR \\
\hline \begin{tabular}{|l} 
Computerwiss. \\
\end{tabular} & 7,56 & 7,14 & 5,13 & 5,02 & 1,61 & 2,44 & 1,34 & 1,26 & 1,27 & 0,92 & 0,74 & 0,82 & 0,35 & 0,32 \\
\hline Ingenieurswesen & 8,76 & 6,59 & 5,14 & 4,12 & 1,75 & 1,98 & 1,50 & 1,45 & 1,03 & 0,64 & 0,60 & 0,60 & 0,45 & 0,27 \\
\hline Materialwiss. & 6,96 & 9,32 & 6,12 & 2,43 & 2,54 & 1,20 & 1,88 & 1,11 & 0,96 & 0,75 & 0,44 & 0,69 & 0,33 & 0,28 \\
\hline Raumwiss. & 14,27 & 13,99 & 10,27 & 8,79 & 5,24 & 4,68 & 1,73 & 1,71 & 1,24 & 1,23 & 1,44 & 0,87 & 0,55 & 0,38 \\
\hline Humanmedizin & UK & GE & FR & IT & $\mathbf{N L}$ & SE & ES & $\mathrm{CH}$ & $\mathrm{BE}$ & FI & DK & AT & NO & IR \\
\hline Klinische Medizin & 10,63 & 8,57 & 5,90 & 4,79 & 3,25 & 2,67 & 2,41 & 2,04 & 1,54 & 1,35 & 1,26 & 1,27 & 0,80 & 0,40 \\
\hline Immunologie & 10,12 & 7,82 & 7,29 & 4,84 & 3,96 & 4,01 & 2,63 & 3,31 & 1,68 & 1,13 & 1,47 & 1,32 & 0,92 & 0,37 \\
\hline \begin{tabular}{|l} 
Neurowiss. \\
\end{tabular} & 10,32 & 8,72 & 6,07 & 5,05 & 2,73 & 2,92 & 2,45 & 2,14 & 1,12 & 1,36 & 0,95 & 0,93 & 0,58 & 0,34 \\
\hline Pharmakologie & 9,20 & 7,65 & 5,70 & 5,01 & 2,77 & 2,47 & 2,91 & 1,83 & 1,55 & 1,18 & 1,06 & 0,81 & 0,54 & 0,27 \\
\hline Agrarwiss./Vetmed. & UK & GE & FR & ES & IT & $\mathrm{NL}$ & SE & $\mathrm{BE}$ & DK & $\mathrm{CH}$ & FI & NO & AT & $\mathbf{I R}$ \\
\hline Agrarwiss. & 7,30 & 6,90 & 5,48 & 4,71 & 3,41 & 2,75 & 1,41 & 1,20 & 1,43 & 1,17 & 1,07 & 0,67 & 0,59 & 0,95 \\
\hline Pflanzenbau/Viehzucht & 9,06 & 7,22 & 5,51 & 3,59 & 2,47 & 2,83 & 2,00 & 1,69 & 1,44 & 1,52 & 1,14 & 1,21 & 0,86 & 0,42 \\
\hline Soz.-\&Wirtschaftswiss. & UK & GE & NL & FR & IT & ES & SE & BE & $\mathrm{CH}$ & FI & NO & DK & IR & AT \\
\hline Sozialwiss. & n.v. & n.v. & n.v. & 1,69 & 0,81 & 0,61 & 1,46 & 0,49 & 0,52 & 0,73 & 0,75 & 0,49 & 0,28 & 0,32 \\
\hline Wirtschaftswiss. & 14,97 & 2,86 & 3,52 & 3,07 & 1,87 & 1,76 & 1,68 & 1,48 & 0,97 & 0,72 & 0,88 & 1,03 & 0,54 & 0,59 \\
\hline Psychologie/Psychiatrie & 11,31 & 6,19 & 3,35 & 2,28 & 1,62 & 1,53 & 1,53 & 0,81 & 0,97 & 0,91 & 0,74 & 0,45 & 0,47 & 0,20 \\
\hline
\end{tabular}

Quelle: Science Citation Index, OECD. Angaben in Prozent. Bevölkerung in absoluten Zahlen. 


\section{Forschungsnetzwerke in Biotechnologie auf europäischer Ebene ${ }^{3}$}

Der folgende Exkurs befasst sich mit der Frage, in welcher Form sich Forschungsnetzwerke in Biotechnologie als Indikator für Selbstorganisation in diesem Bereich auf europäischer Ebene herausbilden. Handelt es sich bei Organisationen, die in Europa zu Biotechnologie forschen, um eine einzige Clique oder um ein ganzes Set von Cliquen? Steigt die Kohäsion oder sinkt sie? Indikator für die Existenz und Häufigkeit von Interaktionen und damit Basis für die Netzwerkmodellierung ist hier die Co-Autorenschaft bei Publikationen zum Thema Biotechnologie auf Länderebene. Co-Autorenschaft zwischen verschiedenen Organisationen eines Landes wird nicht in die Analyse miteinbezogen, ebensowenig wie Co-Autorenschaft innerhalb einer Organisation. Untergruppen (= Cliquen, Cluster) werden identifiziert durch Unterteilung des Netzwerkes in Cluster von relativ hoher Interaktionsdichte. Die Interaktionsdichte ist dann relativ hoch, wenn die Mehrheit der relevanten Verbindungen innerhalb der Untergruppe verbleiben.

\begin{tabular}{|c|c|c|c|c|c|c|c|c|c|}
\hline \multirow[b]{3}{*}{ Jahr } & \multirow{2}{*}{\multicolumn{3}{|c|}{ Anzahl der Publikationen ${ }^{a}$}} & \multicolumn{6}{|c|}{ Anzahl der Publikationen mit Co-Autoren } \\
\hline & & & & \multicolumn{3}{|c|}{ innerhalb der EU } & \multicolumn{3}{|c|}{ in Japan oder den USA } \\
\hline & 1986 & 1992 & 1996 & 1986 & 1992 & 1996 & 1986 & 1992 & 1996 \\
\hline Austria & 4 & 7 & 17 & 0 & 1 & 4 & 1 & 1 & 0 \\
\hline Belgium & 12 & 9 & 18 & 0 & 1 & 6 & 0 & 0 & 0 \\
\hline Denmark & 4 & 6 & 14 & 0 & 0 & 5 & 0 & 0 & 1 \\
\hline Finland & 12 & 9 & 15 & 1 & 1 & 0 & 1 & 2 & 0 \\
\hline France & 64 & 81 & 92 & 1 & 1 & 11 & 2 & 1 & 1 \\
\hline Germany & 49 & 58 & 100 & 2 & 1 & 19 & 4 & 0 & 2 \\
\hline Greece & 5 & 7 & 19 & 0 & 0 & 3 & 0 & 0 & 0 \\
\hline Ireland & 1 & 3 & 14 & 0 & 0 & 1 & 0 & 0 & 0 \\
\hline Italy & 12 & 24 & 40 & 1 & 3 & 5 & 0 & 1 & 0 \\
\hline Netherlands & 27 & 26 & 53 & 0 & 0 & 20 & 0 & 0 & 0 \\
\hline Portugal & 10 & 19 & 29 & 2 & 3 & 9 & 0 & 0 & 1 \\
\hline Spain & 15 & 51 & 91 & 0 & 8 & 16 & 0 & 1 & 3 \\
\hline Sweden & 25 & 17 & 27 & 0 & 0 & 6 & 2 & 0 & 2 \\
\hline UK & 79 & 147 & 138 & 3 & 5 & 23 & 1 & 3 & 4 \\
\hline $\begin{array}{c}\text { Anzahl der } \\
\text { Publikationen in } \\
\text { der EU }\end{array}$ & $314^{b}$ & $418^{b}$ & $617^{b}$ & 5 & 46 & 50 & 11 & 9 & 14 \\
\hline$\%$ & & & & 2 & 11 & 8 & 4 & 2 & 2 \\
\hline
\end{tabular}

a Anzahl der Publikationen, bei welchen zumindest ein Autor eine Adresse in dem betreffenden Land ausweist.

b Die Anzahl der Publikationen in der EU weicht von der Summe der Publikationen der EULänder um die Anzahl der Co-Autorenschaften innerhalb der EU ab.

Quelle: Science Citation Index. Aus: Van den Besselaar and Heimeriks (2000).

In der Folge handelt es sich um Ergebnisse aus: Van den Besselaar, Peter/ Heimeriks, Gaston (2000) Codification and Self-Organization of the European Science-Technology-Innovation System. The Self-Organization of the European Information Society. A research project funded by the European Commission under the TSER program. Final report of task 4, May 2000. 
Die obige Tabelle gibt einen Überblick über das europäische Gemeinschaftspublikationen zu Biotechnologie im Zeitraum zwischen 1986 und 1996. Es wird deutlich, dass die Gesamtzahl der Publikationen innerhalb der Dekade von 314 auf 617 beträchtlich gestiegen ist. Ebenso ist die Anzahl der Publikationen mit internationaler (innerhalb von Europa) Co-Autorenschaft von 5 auf 50 stark gestiegen. Auf der anderen Seite ist die Anzahl der co-publizierten Artikel mit Autoren aus Japan und den USA in etwa konstant geblieben, was relativ ein starkes Absinken bedeutet. Diese Ergebnisse deuten bereits auf die Herausbildung eines europäischen Netzwerkes hin, obwohl die Anzahl der innerhalb von Europa internationalen Co-Autorenschaften noch immer relativ gering ist.
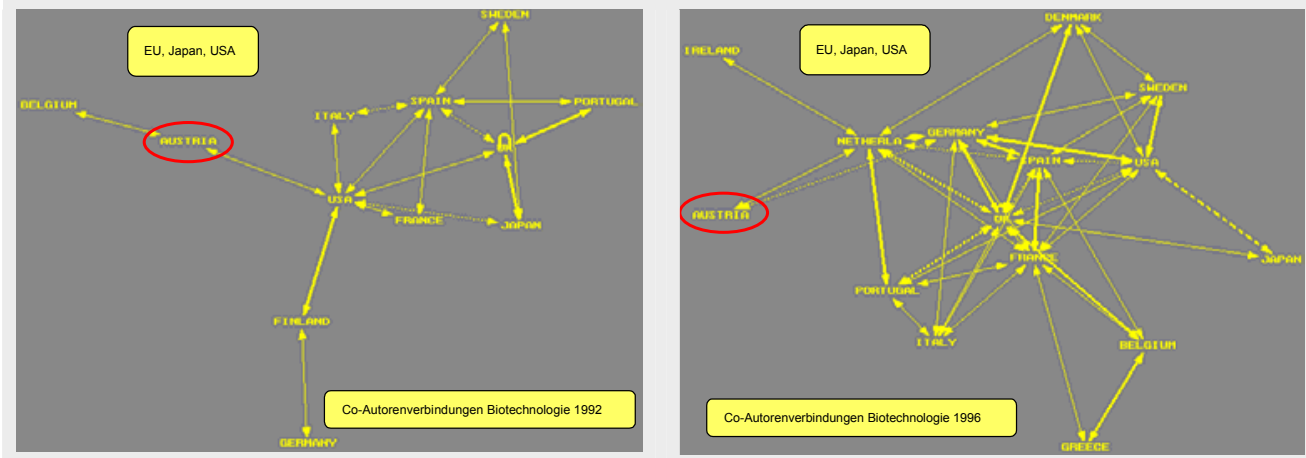

Quelle: Van den Besselaar and Heimeriks (2000)

Für die Analyse der Zeitpunkte 1986, 1992 und 1996 gilt: Im Jahr 1986 (keine Abbildung) ist ein gesamteuropäisches Netzwerk im Bereich Biotechnologie nicht erkennbar. Nur wenige Länder weisen Co-Publikationen mit Autoren aus anderen Ländern auf. Die USA und Japan haben eine zentralere Position im Netzwerk als irgendein europäisches Land.

Für das Jahr 1992 identifiziert eine Clusteranalyse der Co-Autorenschaften vier: Österreich und Belgien sind zusammen in der ersten Clique, Spanien, Großbritannien und Portugal in einer anderen Clique, Japan und USA in einer dritten und Finnland, Deutschland, Italien und Schweden in einer vierten Clique. Das Ergebnis dieser Clusteranalyse und die Visualisierung der Beziehungen auf Basis der Co-Autorenschaften (siehe Abbildung oben) suggerieren, dass ein innereuropäisches Netzwerk in Biotechnologie im Jahr 1992 möglicherweise im Begriff ist, sich herauszubilden. Akteure an zentraler Position im Netzwerk sind im Jahr 1992 noch immer Japan und die USA, aber auch ein EU-Cluster - nämlich GB, Spanien und Portugal.

Für das Jahr 1996 weist eine hierarchische Clusteranalyse auf starke, geographisch bedingte Cliquenbildung hin: Schweden und Dänemark befinden sich in einem Cluster, Italien, Spanien und Frankreich in einem weiteren, Deutschland, Österreich und die Niederlande in einem dritten. Die USA und Japan bilden noch immer einen eigenen Cluster. Auch die Netzwerk der Co-Autoren (Abb. oben rechts) weist auf eine zunehmend engere Beziehung zwischen einigen europäischen Ländern hin. Zusammenfassend lässt sich sagen, dass sich im Jahr 1996 ein innereuropäisches Netzwerk in Biotechnologie bereits stärker entwickelt hat. Obwohl die Gesamtanzahl der innereuropäischen Co-Autorenschaften noch immer relativ gering ist, so ist sie doch in den Jahren davor stark angewachsen. Die USA und Japan befinden sich im Jahr 1996 von ihrer Positionierung und von der Anzahl ihrer Relationen her außerhalb des europäischen Netzwerkes. Die EU scheint sich nicht als ein einziges Netzwerk herauszubilden, sondern zerfällt in geographische und kulturelle Untergruppen. 


\subsection{Humanressourcen}

Neben der Forschung zählt die Ausbildung zu den Hauptaufgaben des Bildungssystems. Die verstärkte Nachfrage nach hochqualifizierten Arbeitskräften hat auch zu verstärkten Anreizen für den Einzelnen geführt, Bildungsangebote in Anspruch zu nehmen. In der Europäischen Union hat sich die Zahl der Studierenden an Einrichtungen des tertiären Bereichs im Laufe der letzten zwanzig Jahren mehr als verdoppelt. Österreich kann einen Anstieg um das 2,5fache seit 1975 verzeichnen. In Zeiten der zunehmenden Anforderung nach „Lebenslangem Lernen“ besteht somit die Aufgabe darin, einerseits der hohen Nachfrage gerecht zu werden, und andererseits die für eine Wissensgesellschaft notwendigen Kompetenzen zu fördern. Der Bildungsstand einer Bevölkerung ist eine häufig verwendete indirekte Kennzahl für den Bestand des Humankapitals, d.h. für alle in der Bevölkerung und der Erwerbsbevölkerung verfügbarer Kenntnisse und Fähigkeiten.

\section{Abbildung 3-7: Verteilung der Bevölkerung im Alter von 25 bis 64 Jahren nach Bildungsstand (1999) ${ }^{4}$}

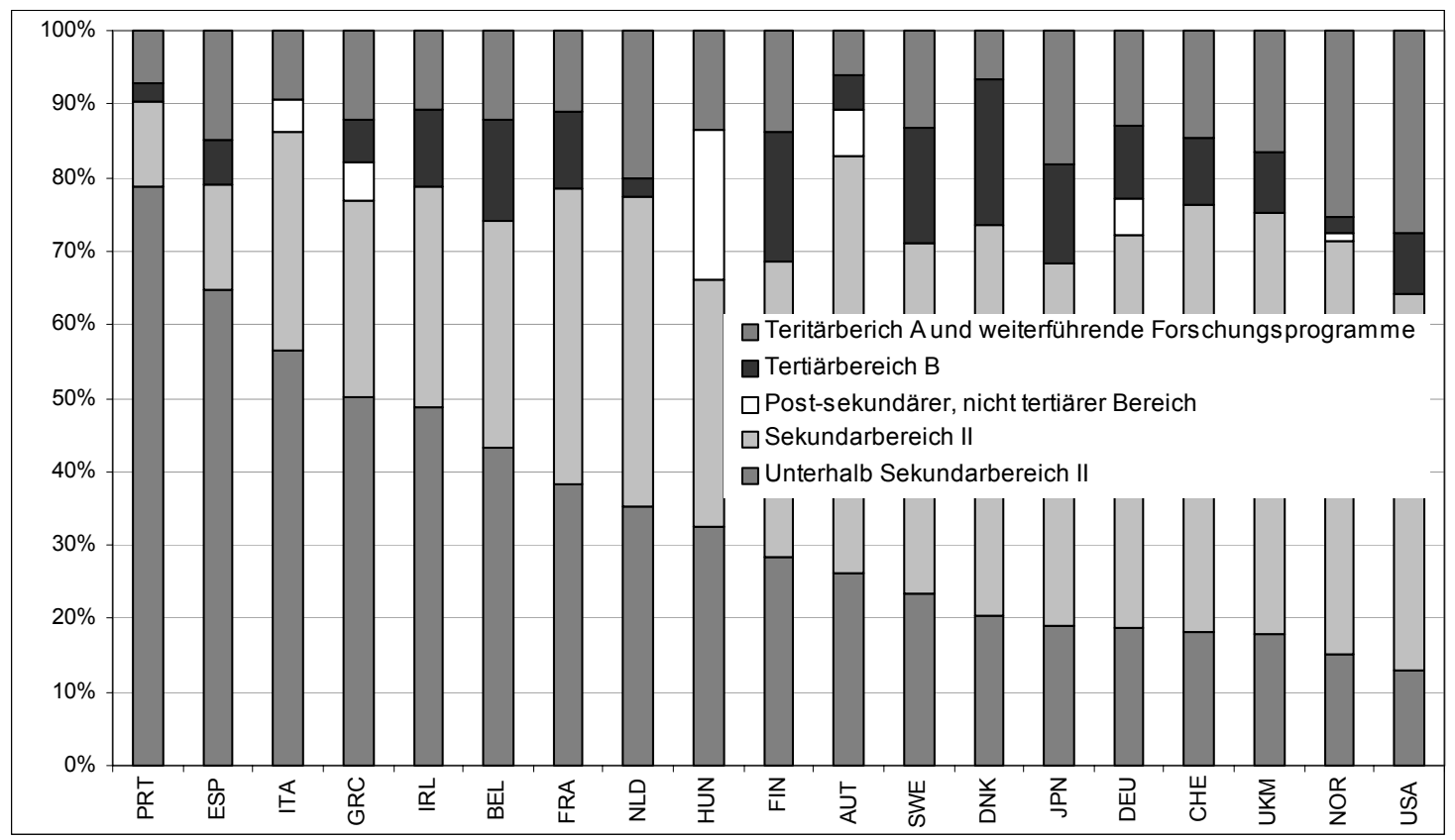

Quelle: OECD 2001c

Sekundarbereich II: Die Bildung in diesem Bereich beginnt für Schüler im Alter von ca. 14 oder 15 Jahren, und umfasst allgemeinbildende, berufliche oder fachspezifische Bildungsgänge. Die Bildungsgänge führen entweder zum Erwerb einer Zugangsberechtigung zum Tertiärbereich oder zu einer schulischen Abschlussqualifikation.

Tertiärerbereich A: sind weitgehend theoretisch orientiert und sollen hinreichende Qualifikationen für den Zugang zu weiterführenden Forschungsprogrammen und Berufen mit hohem Qualifikationsniveau vermitteln (Hochschulstudium).

Tertiärbereich B: sind typischerweise kürzer als im Tertiärbereich A und konzentrieren sich auf praktische/technische/berufsbezogene Fähigkeiten für den direkten Eintritt in den Arbeitsmarkt (Fachhochschulen). 
Abbildung 3-7 zeigt den Bildungsstand der Bevölkerung zwischen 25 bis 64 Jahren im Jahre 1999. Dabei zeigt sich, dass der Anteil der 25- bis 63-Jährigen, die einen Abschluss des Tertiärbereichs $A$ bzw. in einem weiterführenden Forschungsprogramm haben, in den OECDLändern von weniger als 10 Prozent in Dänemark, Italien, Österreich und Portugal bis auf 20 Prozent und mehr in den Niederlanden, Norwegen und den USA reicht. Für Österreich gilt, dass 74\% der Bevölkerung mindestens einen Abschluss im Sekundarbereich aufweisen. Österreich liegt damit etwa im Mittelfeld der untersuchten Länder. Allerdings unterscheidet sich diese Quote zwischen den einzelnen Altergruppen deutlich. Weisen 83\% der 25 - 33-jährigen zumindest einen Abschluss im Sekundarbereich auf, so sinkt der Anteil bei den 55 - 63-jährigen auf 59\% (OECD, 2001). Dies kann als ein Zeichen dafür gewertet werden, dass steigende Anforderungen des Arbeitsmarktes an die Qualifikation der Arbeitnehmer den Anteil junger Menschen erhöht haben, die zumindest einen Abschluss im Sekundarbereichs vorweisen können.

Bildung als Investition in das Humankapital trägt wesentlich zum Wirtschaftswachstum und Produktivitätssteigerungen bei. Neben dem gesamtwirtschaftlichem Nutzen entstehen für die öffentliche Hand als hauptsächlichem Anbieter aber auch Kosten, die durch die gesteigerte Nachfrage nach höherwertiger Bildung in den meisten Industriestaaten in den 90er Jahren wesentlich gestiegen sind. Vor allem im Tertiärbereich konnten die aufgewendeten Mittel oft nicht mit dem raschen Anstieg der Studienzahlen mithalten. Dennoch sind die Ausgaben pro Schüler/Studierendem gemessen am BIP pro Kopf ein Maßstab für die Bildungsausgaben und für den relativen Wohlstand eines Landes.

Abbildung 3-8: Ausgaben pro StudentIn im Verhältnis zum BIP pro Kopf (1998)

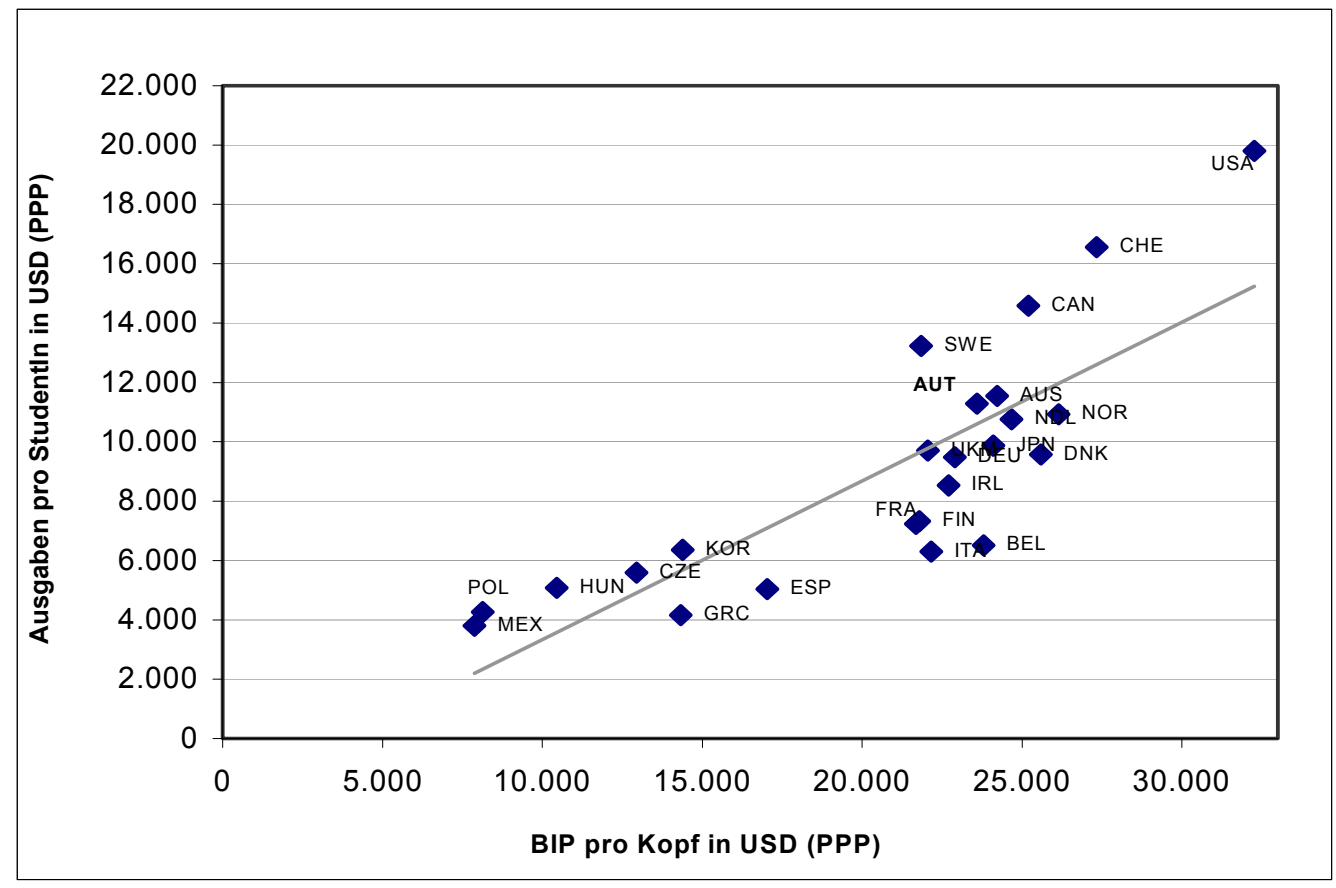

Quelle: OECD 2001c, eigene Berechnungen 
Wie in der Grafik zu erkennen ist, besteht ein eindeutiger Zusammenhang zwischen Ausgaben pro Schüler/Studierenden einerseits und dem BIP pro Kopf andererseits. Gemessen am BIP pro Kopf geben ärmere Länder tendenziell relativ weniger pro Studenten aus als reichere Länder. Unter Berücksichtigung sowohl der öffentlichen wie auch der privaten Finanzquellen investieren die OECD-Länder 5,7\% des BIP (1998) in Bildungseinrichtungen. Österreich konnte eine Steigerung der Bildungsausgaben von 5,1\% des BIP im Jahre 1990 und 6,61\% des BIP im Jahre 1995 auf 6,36\% des BIP im Jahre 1998 verzeichnen. Österreich liegt damit über dem OECD-Durchschnitt.

Durchschnittlich entfallen in den untersuchten Ländern mehr als ein Viertel der gesamten Bildungsausgaben auf Einrichtungen des Tertiärbereichs. Die USA investieren 2,3\% des BIP in den Tertiärbereich und liegen damit an der Spitze. Österreich liegt mit 1,46\% des BIP gleichauf mit Dänemark und Norwegen. Europäische Spitzenreiter sind Finnland und Schweden mit jeweils $1,67 \%$ des BIP. Trotz des relativ hohen BIP-Anteils, welcher in den Tertiärbereich investiert wird, verzeichnet Österreich nur einen relativ geringen Bevölkerungsanteil mit tertiärem Bildungsabschluss. Auch wird dieses Ergebnis nicht durch einen überdurchschnittlichen Forschungsoutput kompensiert, was die Anzahl an Publikationen in Relation zu den Ausgaben für Forschung und Entwicklung im Tertiärbereich, als ein möglicher Outputindikator, zeigt.

\section{Abbildung 3-9: Bildungsstand im Verhältnis zu Ausgaben und Forschungsoutput, 1998}

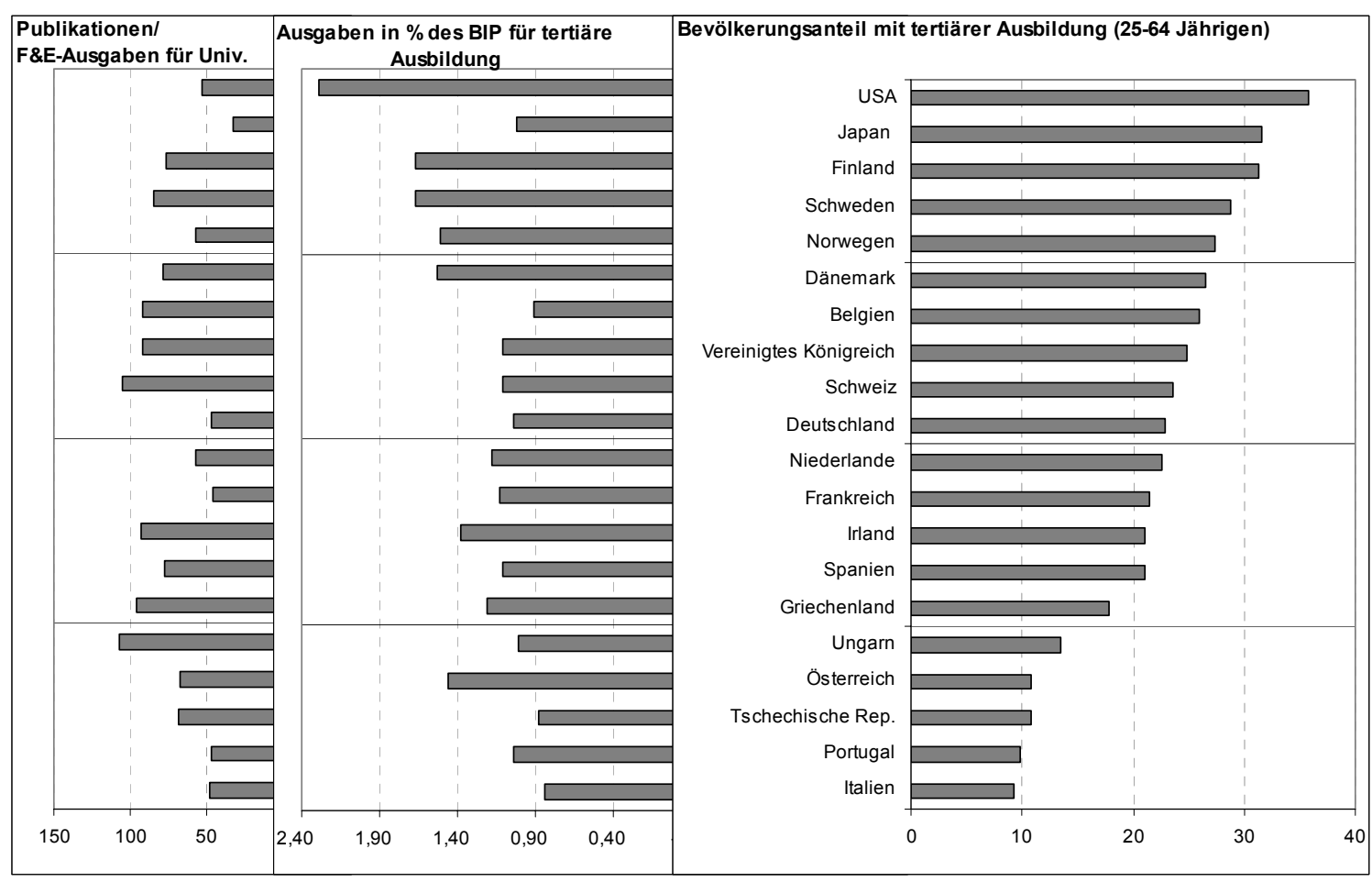

Quelle: OECD 2001c, eigene Berechnungen 


\section{Die Internationalisierung des österreichischen Forschungs- und Innovationssystems}

\subsection{Die österreichische Beteiligung am 5. Rahmenprogramm}

\subsubsection{Die Struktur des 5. RP}

Das 5. Rahmenprogramm (RP) wurde im Dezember 1998 vom Europäischen Rat der Forschungsminister beschlossen. Mit einem Gesamtbudget von 14,96 Mrd. € für die Laufzeit 1998-2002 weist das 5. RP eine Mittelerhöhung gegenüber dem 4. RP von 4,61\% aus und unterscheidet sich auch inhaltlich und strukturell in einigen Punkten vom 4. RP. Hauptziel des 5. $\mathrm{RP}$ ist es, zur Lösung wirtschaftlicher und sozialer Probleme beizutragen („problemorientierter Ansatz"), wodurch es auch zu einem Ausbau der Multidisziplinarität sowie zu einer festen Verankerung der sozio-ökonomischen Forschung in den spezifischen Programmen kam.

Tabelle 4-1: Das 5. Rahmenprogramm - Budgetäre Mittelaufteilung nach Programmen

\begin{tabular}{llr}
\hline Indirekte Aktion & & Mill.€ \\
\hline 1. Aktionslinie & Thematische Programme & $\mathbf{1 0 . 8 4 3}$ \\
& Lebensqualität und Management lebender Ressourcen (QoL) & 2.413 \\
& Benutzerfreundliche Informationsgesellschaft (IST) & 3.600 \\
& Wettbewerbsorientiertes und nachhaltiges Wachstum (GROWTH) & 2.705 \\
& Umwelt und nachhaltige Entwicklung (UW) & 1.083 \\
& Energie (EN) & 1.042 \\
2. Aktionslinie & Horizontale Programme & 475 \\
3. Aktionslinie & Innovation und Einbeziehung der KMU (INNO/SME) & 363 \\
4. Aktionslinie & Humanressourcen u. sozio-ökonomische Wissensgrundlage (IHP) & 1.280 \\
\hline Direkte Aktion & & 739 \\
\hline & Joint Research Centre (JRC) & 1.260 \\
\hline & EURATOM & $\mathbf{1 4 . 9 6 0}$ \\
\hline
\end{tabular}

Quelle: CORDIS

Die thematischen Programme umfassen jeweils drei Arten von Aktionen: Die Leitaktionen dienen dazu, Forschungsmaßnahmen auf die Bereiche zu konzentrieren, die wichtige wirtschaftliche und gesellschaftliche Anliegen betreffen. Auf sie entfallen nahezu 85\% der Mittel der thematischen Programme. Die generischen FTE-Tätigkeiten ergänzen die Leitaktionen und betreffen vorrangige Forschungstätigkeiten, die eher langfristig ausgerichtet sind und deren Ergebnisse breitgefächerte Anwendungen umfassen. Ziel der Maßnahmen zur Förderung der Forschungsinfrastruktur ist schließlich, den Zugang zu den Forschungseinrichtungen zu verbessern und ihre europaweite Vernetzung zu fördern. Die horizontalen Programme erfüllen Querschnittsaufgaben und umfassen spezifische Maßnahmen, die durch die thematischen 
Programme noch nicht abgedeckt sind sowie Koordinierungs-, Unterstützungs- und Begleitmaßnahmen, die die Kohärenz gleichartiger spezifischer Maßnahmen in den einzelnen thematischen Programmen sicherstellen sollen.

\subsubsection{Die österreichische Beteiligung im internationalen Vergleich}

Da sich das 5. RP im letzten Drittel seiner Laufzeit befindet, können noch keine endgültigen Zahlen zur österreichischen Beteiligung präsentiert werden. Dennoch lässt sich auf Basis der vorhandenen Zahlen bereits sagen, dass sich sowohl die Präsenz als auch die Rückflussraten für Österreich im 5. RP sehr zufriedenstellend entwickeln. Mit Stand März 2002 waren österreichische Partner an 1.086 Projekten beteiligt, das sind nahezu $11 \%$ aller EU-weit geförderten Projekte.

Abbildung 4-1: Österreich im 5. RP: Anzahl der Projekte

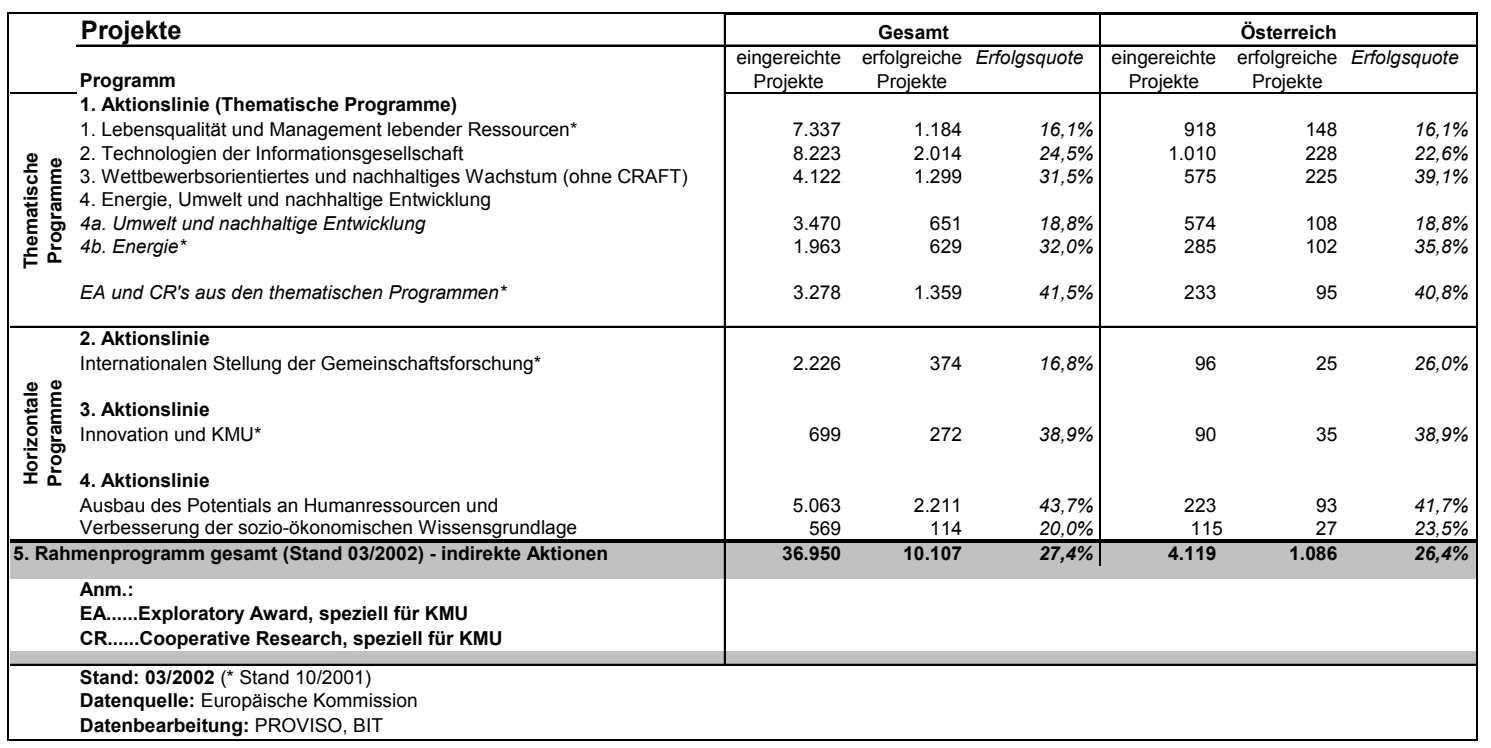

Quelle: Europäische Kommission; Berechnungen BIT, PROVISO

Die Erfolgsquote der Projekte mit österreichischer Beteiligung liegt mit 26,4\% leicht unter dem europäischen Durchschnitt. Allerdings lässt sich im Programm „Wettbewerbsorientiertes und nachhaltiges Wachstum" mit 39,1\% auch eine Erfolgsquote beobachten, die weit über dem europäischen Durchschnitt liegt. Ebenfalls deutlich über dem europäischen Durchschnitt liegen die Projekte mit österreichischer Beteiligung im INCO-Programm.

Vergleicht man das 5. RP mit dem 4. RP, so lässt sich eine deutliche Bündelung der Mittel hinsichtlich des Umfangs der finanzierten Projekte feststellen: Das durchschnittliche Projektvolumen hat sich gegenüber dem vorherigen RP nahezu verdoppelt und beträgt durchschnittlich 1,5 Mill. $€$ bei den thematischen Programmen. 
Auf der Ebene der Beteiligungen weist Österreich (Stand 03/2002) 1.531 Beteiligungen auf, was einen Anteil von $2,4 \%$ an allen Beteiligungen ergibt. Auf europäischer Ebene ergibt das durchschnittlich 6,5 Beteiligungen pro Projekt.

Abbildung 4-2: Österreich im 5. RP: Anzahl der Beteiligungen

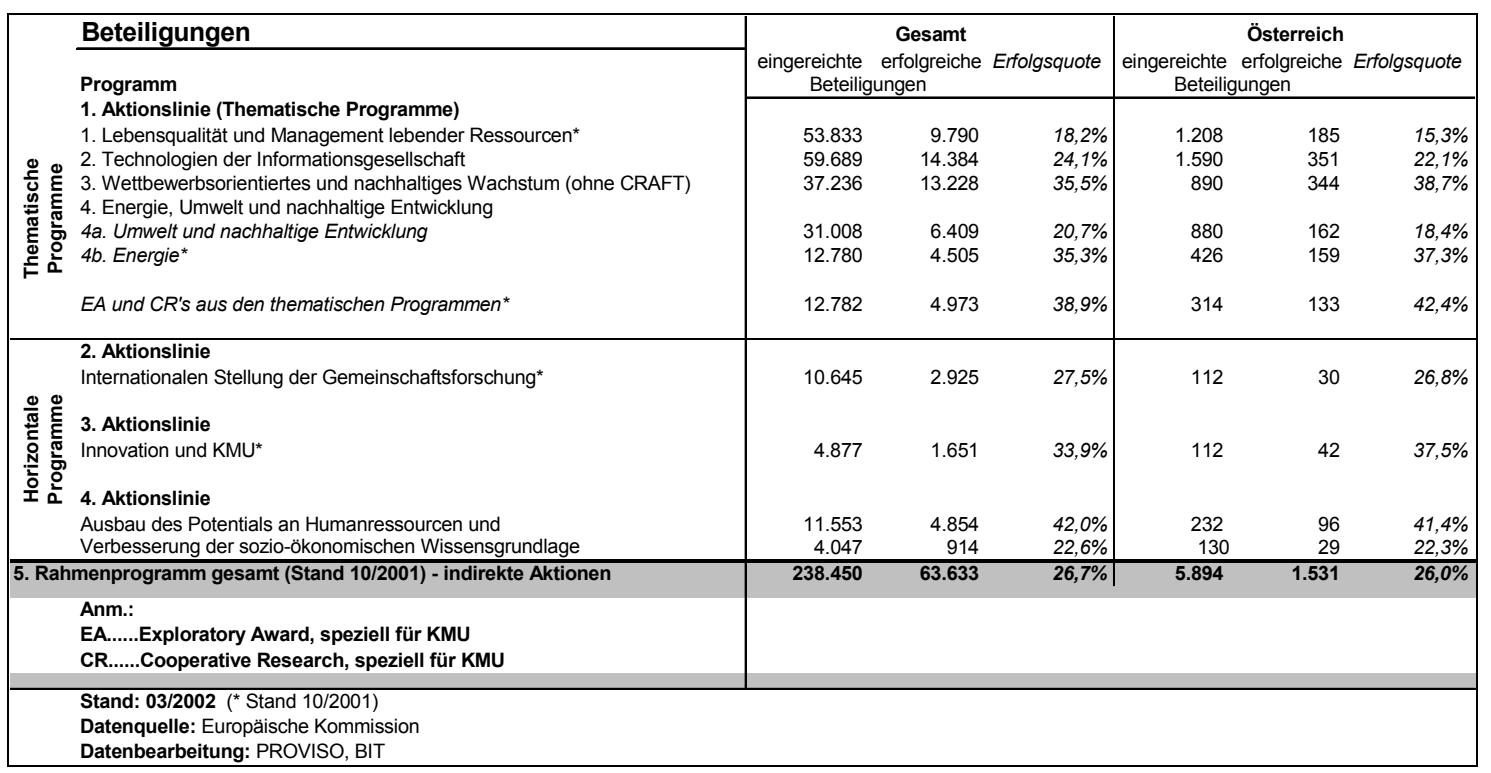

Quelle: Europäische Kommission; Berechnungen BIT, PROVISO

\subsubsection{Förderungen aus dem 5. Rahmenprogramm}

Mit Stand Oktober 2001 erhielten österreichische Partner aus den Programmen Benutzerfreundliche Informationsgesellschaft (IST) Wettbewerbsorientiertes und nachhaltiges Wachstum (GROWTH), Energie (EN), Umwelt und nachhaltige Entwicklung (UW) und Ausbau des Potentials an Humanressourcen in der Forschung und der Verbesserung der sozioökonomischen Wissensgrundlage (IHP) sowie Quality of Life (QoL) über 200 Mill. $€$ an Förderungen zugesprochen. Dies entspricht einem nationalen Anteil von 2,58\% an den bisher zugesagten Förderungen in diesen Programmen (Topolnik et al. 2001). Vergleicht man die österreichische Rückflussrate aus dem 5. RP mit dem aus dem 4. RP, so bedeutet dies eine deutliche Verbesserung der Ergebnisse: Der österreichische Anteil an den Förderungen aus dem 4. RP betrug jährlich zwischen 1,5 und 1,7\%, was deutlich unter dem aktuellen Anteil aus dem 5. RP (2,58\%) liegt. Umgelegt auf die österreichischen Beitragszahlungen entsprachen die Förderungen im 4. RP ca. $60 \%$ des österreichischen Beitrages zum gesamten 4. RP (via EUHaushalt). Für das 5. RP beträgt die Deckungsquote derzeit bereits $99 \%$, wodurch sich Österreich in Richtung finanzieller Ausgeglichenheit von Beitragszahlungen und Rückflüssen bewegt (vgl. Topolnik et al. 2001).

Ein Ländervergleich zeigt weiters, dass Österreich mit einem Anteil von rund 2,6\% an der Gesamtfördersumme zwar seinen Anteil an den Bruttobeitragszahlungen zum EU-Haushalt abdeckt, jedoch andere Länder wie Schweden oder Dänemark einen höheren Anteil an der 
Gesamtfördersumme aufweisen. Dennoch hat sich Österreich sehr positiv entwickelt. Lag Österreich gemessen an den gesamten Rückflüssen aus dem 4. RP noch gleichauf mit Irland (Schibany et al, 2001) so ist die Performance Österreichs im 5. RP deutlich besser. Ein weiteres interessantes Ergebnis ist der relativ geringe Anteil Finnlands. Sowohl auf Ebene der erfolgreichen Projekte als auch in absoluten Rückflüssen liegt Finnland im 5. RP hinter Österreich. Obwohl Finnland mit 3,3\% eine sehr hohe Forschungsquote aufweist, zeigt es eine erstaunlich geringe Präsenz in den Rahmenprogrammen.

\section{Abbildung 4-3: 5. RP - Förderungen nach Ländern versus Beitragszahlungen zum EU- Haushalt $^{5}$; (EU 15=100\%), Stand 10/2001}

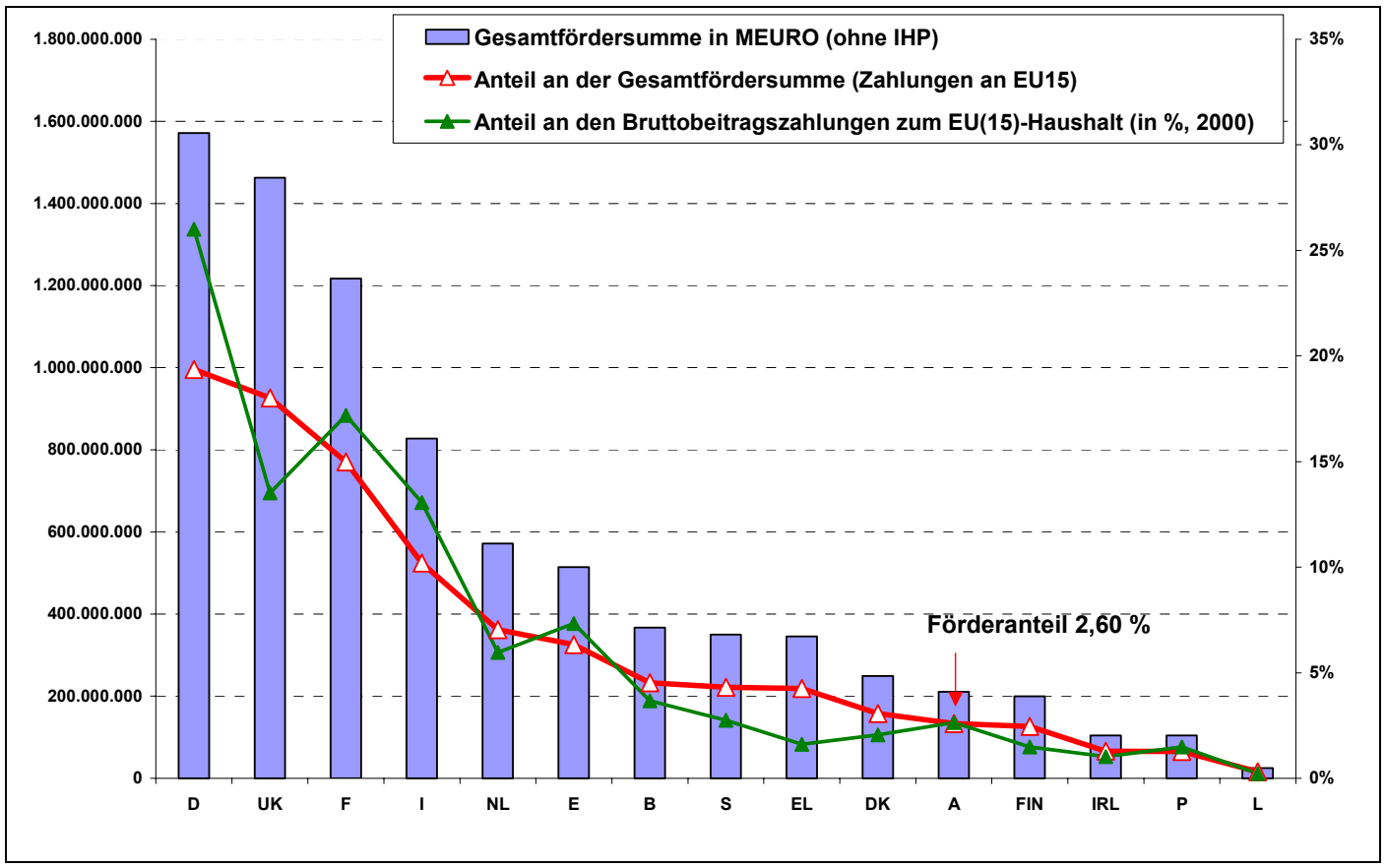

Quelle: Europäische Kommission; Berechnungen PROVISO

\subsection{4 Österreichische Koordinatoren im 5. RP}

Insgesamt wurden bislang 234 Projekte von österreichischen Einrichtungen koordiniert. Bei 920 von österreichischen Koordinatoren eingereichten Projekten ergibt das eine mittlere Erfolgsrate von $25,4 \%$ wobei diese Rate zwischen den Programmen divergiert. Vergleicht man alle Programme, so wies Österreich in den Programmen "Growth“ und „IHP“ die höchste absolute Zahl an Koordinatoren auf. In diesen Programmen ist auch die Erfolgsrate am höchsten, am niedrigsten ist die Erfolgsrate in QoL mit 14,7\% und UW mit 14,3\%. Setzt man die Gesamtzahl der eingereichten Projekte (4.119) in Beziehung zu der Anzahl der von österreichischen Koordinatoren eingereichten Projekte (920), so ergibt das einen Anteil von etwa $22 \%$ aller

5 Die Daten berücksichtigen die Programme IST, Growth, Umwelt, Energie, LA Soziökonomie, QoL, nicht jedoch das Programm IHP. 
eingereichten Projekte, die von Österreichern auch koordiniert werden. Auf der Ebene der erfolgreichen Projekte werden 22\% aller Projekte mit österreichischer Beteiligung auch von österreichischen Forschern oder Forscherinnen koordiniert.

Abbildung 4-4: Österreichische Koordinatoren

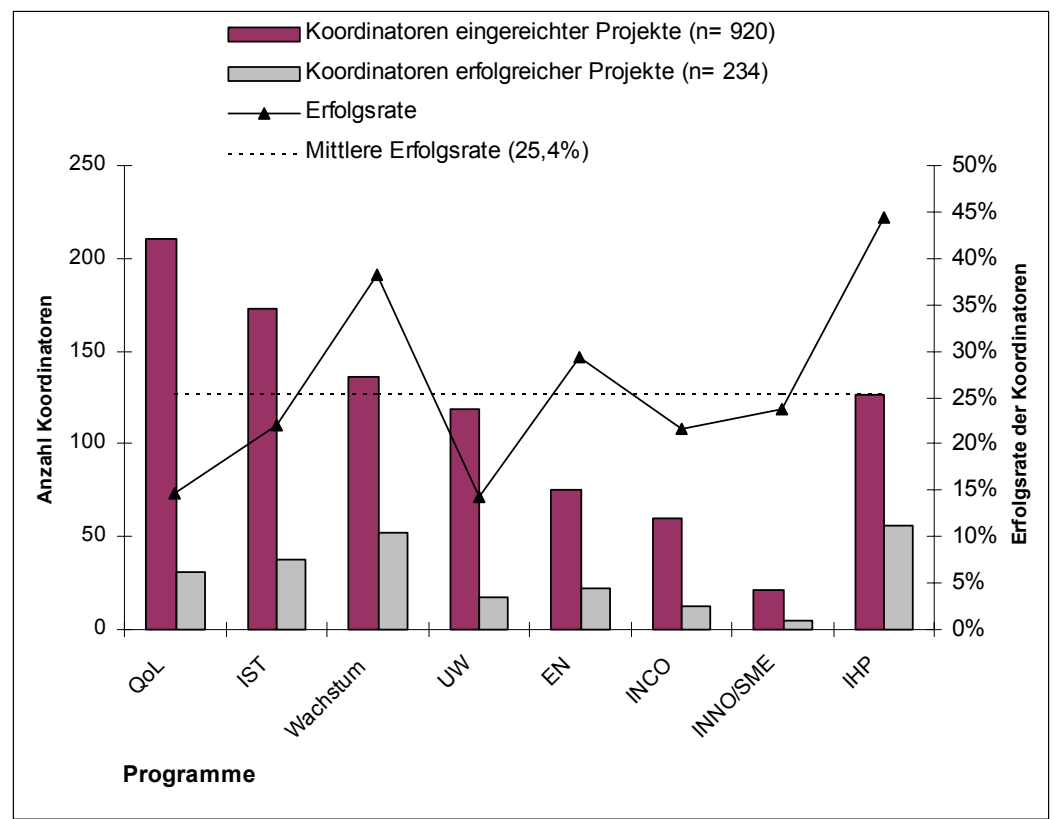

Quelle: Europäische Kommission; Berechnungen BIT

\subsubsection{Die österreichische Beteiligung nach Organisationstypen}

Auf der Ebene der Beteiligungen in erfolgreichen Projekten macht der Anteil der Unternehmen $37 \%$, jener der Universitäten $32 \%$ und der Forschungseinrichtungen $16 \%$ aller österreichischen Beteiligungen aus. Verglichen mit dem 4. RP lässt sich somit eine Annäherung zwischen der Industrie und dem Hochschulbereich in der Zahl der Beteiligungen beobachten, wobei nach wie vor der Unternehmenssektor den höheren Anteil aufweist. Dies ergibt sich hauptsächlich durch die höhere Erfolgsquote der Unternehmen (29\%), da bei den eingereichten Beteiligungen der Anteil des universitären Bereichs leicht über jenem des industriellen Bereichs liegt.

Auf Ebene der Programme zeigen die Beteiligungen nach Organisationstypen naturgemäß ein sehr unterschiedliches Bild. So ist in absoluten Zahlen die Industrie im Wachstumsprogramm am stärksten vertreten, gefolgt vom IST-Programm und EN. In anderen Programmen wie QoL zeigen die Universitäten eine starke Präsenz: nahezu 60\% aller österreichischen Beteiligungen in diesem Programm entfallen auf österreichische Universitäten. Im Umweltprogramm macht der Anteil der Universitäten 50\% an allen österreichischen Beteiligungen aus. 


\section{Abbildung 4-5: Österreichische Beteiligung nach Organisationstypen}

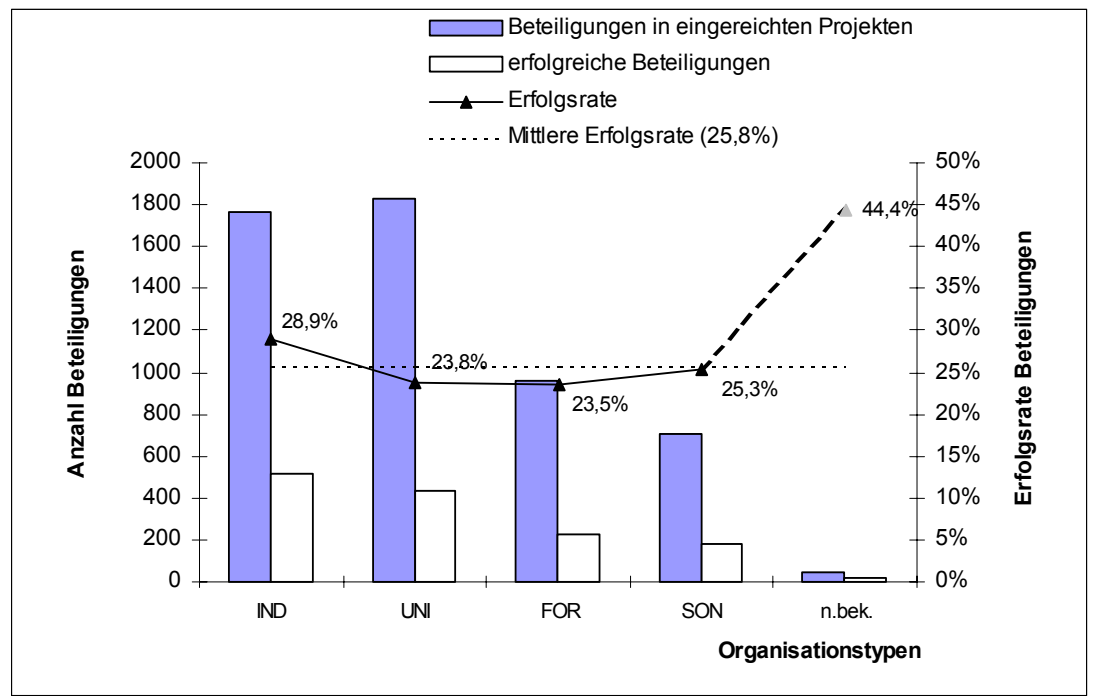

Quelle: Europäische Kommission; Berechnungen BIT

Abbildung 4-6: Anteil verschiedener Organisationstypen an erfolgreichen österreichischen Beteiligungen

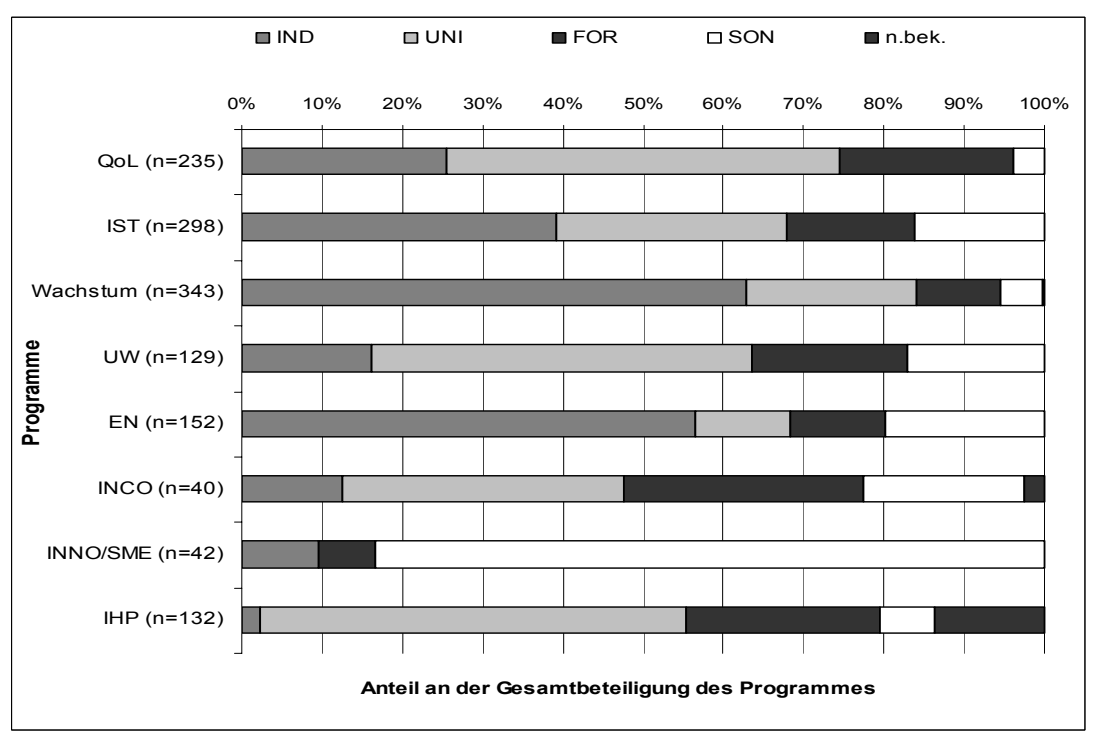

Quelle: Europäische Kommission; Berechnungen BIT

\subsubsection{Neue Teilnehmer im 5. RP}

Es ist eine vieldiskutierte Frage, inwieweit die Europäischen Rahmenprogramme neue Kooperationen zwischen den Teilnehmern stiften konnten oder ob sich über die Zeit eine relativ 
klar definierte Klientel herausgebildet hat, die die notwendigen Voraussetzungen erfüllt und Erfahrungen mitbringt, sich mehrfach an den Rahmenprogrammen zu beteiligen. Diese Fragestellung wird angesichts der geplanten neuen Instrumente und Strukturen im 6. RP in Zukunft für Österreich von höchster Relevanz sein. Auf Basis der vorhandenen Daten lässt sie sich für Österreich klar beantworten: Im 5. RP konnte neues Potential erschlossen werden, womit sich auch der Kreis jener Organisationen, welche Erfahrungen mit den Europäischen Rahmenprogrammen haben, deutlich ausgeweitet hat. Insbesondere im Unternehmenssektor ist der Anteil der Antragsteller, die sich erstmals im 5. RP beteiligt haben („Newcomer“), mit über $69 \%$ ausgesprochen hoch. $30 \%$ der im 5. RP teilnehmenden Unternehmen haben dagegen auch Erfahrungen im 4. RP gemacht.

Abbildung 4-7: Newcomers vs. erfahrene Teilnehmer im 5. RP

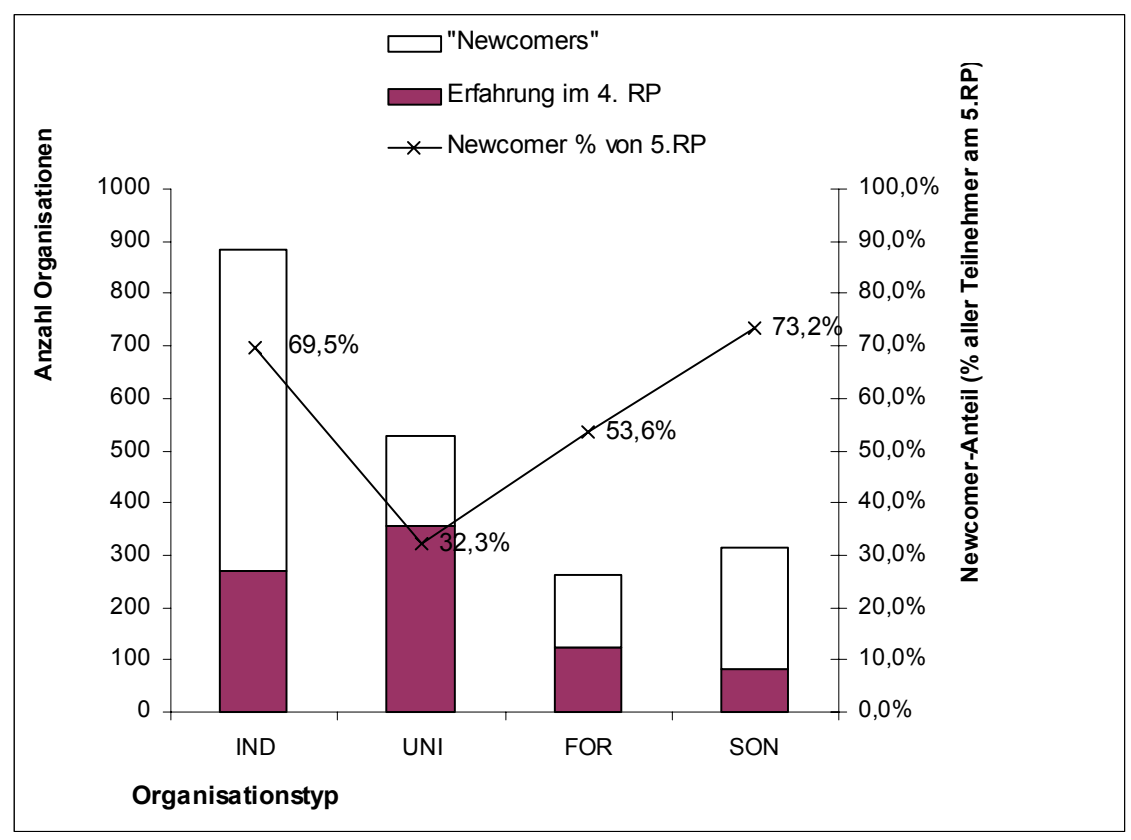

Quelle: Europäische Kommission; Berechnungen BIT

Anders sieht die Situation bei den Universitäten aus. Universitätsinstitute zeigen traditionell eine starke Präsenz in den Rahmenprogrammen, wodurch der Anteil der "Newcomers" dementsprechend geringer ausfällt. Knapp zwei Drittel aller am 5. RP teilnehmenden Universitätsinstitute haben sich bereits am 4. RP beteiligt. Der Sektor der außeruniversitären Forschungseinrichtungen weist einen Newcomer-Anteil von 54\% auf, und aus der Gruppe der sonstigen Organisationen nehmen immerhin mehr als zwei Drittel erstmals am RP teil.

Die Beteiligung an den Europäischen Rahmenprogrammen erfordert auch einen professionellen Umgang mit spezifischen Spielregeln, wobei hier naturgemäß Erfahrungen aus früheren Teilnahmen eine große Rolle spielen. Diese Beobachtung lässt sich an den unterschiedlichen Erfolgsquoten zwischen den Newcomers im 5. RP und jenen Organisationen verifizieren, die schon im 4. RP Erfahrungen sammeln konnten. Es zeigt sich über alle Organisationstypen, 
dass bereits vorhandene Erfahrungen mit den Rahmenprogrammen die Wahrscheinlichkeit eines erfolgreichen Projektantrages erhöhen (siehe Abbildung 4-8).

\section{Abbildung 4-8: Unterschiede in den Erfolgraten}

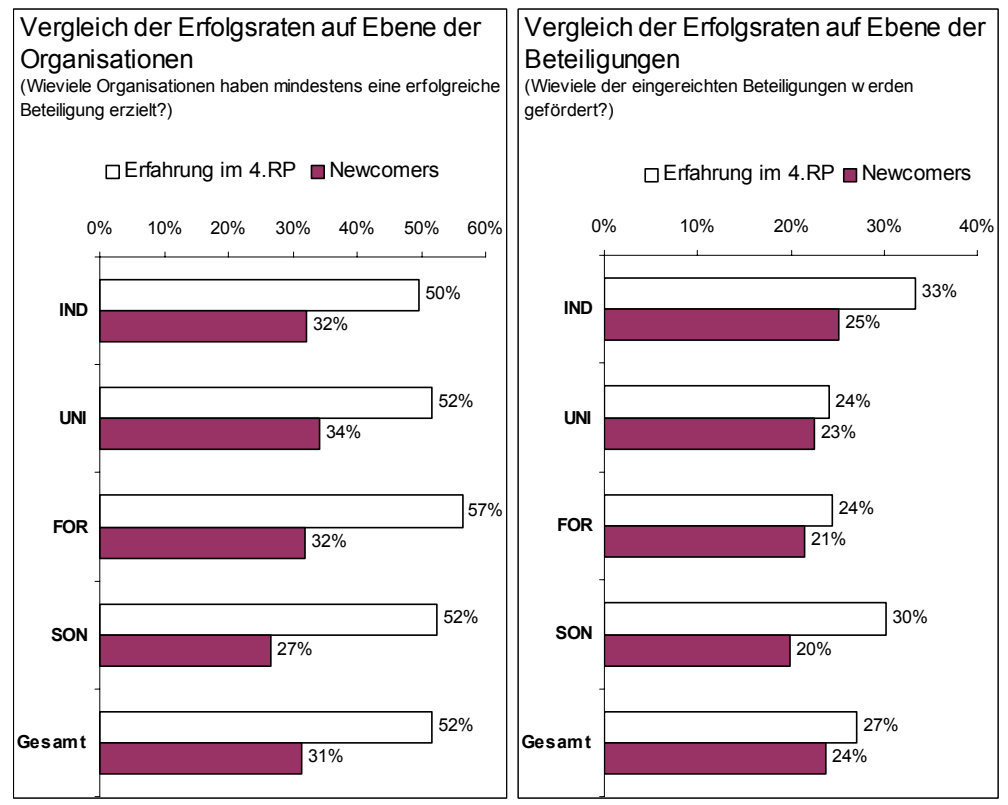

Quelle: Europäische Kommission; Berechnungen BIT

Versucht man, sämtliche bislang in den Europäischen Rahmenprogrammen einreichenden Organisationen zu gruppieren und zwar in solche, die ausschließlich im 4. oder im 5., und solchen, die in beiden Rahmenprogrammen eingereicht haben, ergibt sich eine relativ kleine Schnittmenge (siehe Abbildung 4-9). Vor allem im Unternehmenssektor gibt es relativ wenig teilnehmende Organisationen, die bislang in beiden Rahmenprogrammen eingereicht haben. Dies erklärt vor allem den hohen Anteil an Newcomern im 5. RP. Eine wichtige Frage, die sich daraus ergibt, lautet: Warum haben Unternehmen, die im 4. RP einen Projektantrag eingereicht haben, sich nicht auch im 5. RP beworben? Zur Beantwortung dieser Fragestellung bedarf es einer genaueren Analyse. Dennoch lassen sich drei mögliche Erklärungen nennen:

- Der Aufwand der Teilnahme an EU-Projekten ist sehr hoch, wodurch sich ein negativer Anreiz für eine zukünftige Beteiligung an den Rahmenprogrammen ergibt.

- Die Abwicklung von Projekten, die in der zweiten Hälfte der Programmlaufzeit des 4. RP eingereicht wurden, fällt in den Zeitraum des 5. RPs. Cet.par. ist im 5. RP mit einer geringeren Beteiligung von diesen Unternehmen zu rechnen.

- Die dritte und wahrscheinlichste Erklärung kann auf der Basis der Evaluierung der österreichischen Beteiligung im 4. RP gefunden werden (siehe Schibany et al., 2001). Die vorwettbewerbliche Ausrichtung des 4 . RP sowie die generell sehr positive Einschätzung des Nutzens einer Beteiligung seitens der teilnehmenden Organisationen lässt die ökonomische Verwertungsphase der Ergebnisse nach Beendigung des Projektes einsetzen. Diese Erklärung ließe somit auf eine sehr erfolgreiche Projektabwicklung im 4. 
RP schließen; mit einer anschließenden Verwertung während der Laufzeit des 5. RP. Mit konstant bleibenden Ressourcen würde dies ebenfalls eine geringere Beteiligung dieser Gruppe im 5. RP ergeben.

Abbildung 4-9: Einreichende Organisationseinheiten im 4. und 5. RP

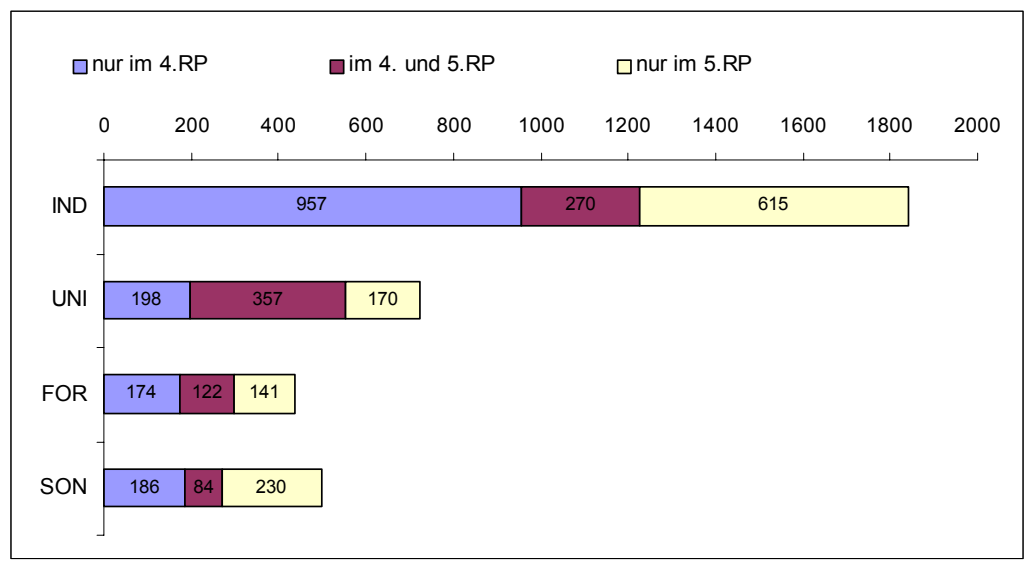

Quelle: Europäische Kommission; Berechnungen BIT

\subsection{Die österreichische Beteiligung in EUREKA}

Die EUREKA-Initiative zur Durchführung marktnaher F\&E Projekte wurde 1985 gegründet, um die Wettbewerbsfähigkeit der europäischen Industrie zu stärken. 30 europäische Länder, Israel und die Europäische Kommission sind Mitglieder dieser Initiative. Zum EUREKA-Netzwerk gehören außerdem 5 (NIP) assoziierte Länder in Ost- und Südosteuropa. Zwei Charakteristika kennzeichnet dieses Programm: (i) Das grundlegende Prinzip des Projektdesigns ist „bottomup“. Das bedeutet, dass die Projektpartner über Inhalt, Beginn, Dauer und Kosten der Projekte selbst entscheiden. Dies ist ein bedeutender Unterschied zu den Rahmenprogrammen, die in Form von Calls jeweils ausgeschrieben werden. EUREKA ist (ii) dezentral organisiert, was bedeutet, dass die Projektteilnehmer jeweils von ihren nationalen Förderinstitutionen gefördert werden, wodurch sich auf europäischer Ebene eine optimale Ergänzung zu den Rahmenprogrammen ergibt. Die bisherigen Aktivitäten im Rahmen der EUREKA-Initiative lassen sich folgendermaßen zusammenfassen:

- Bislang wurden mehr als 2.500 Projekte im Gesamtwert von über $15 \mathrm{Mrd}$. € mit mehr als 7.000 Teilnehmern aus 48 Ländern und der Europäischen Kommission abgeschlossen bzw. befinden sich im Durchführungsstadium.

- Derzeit laufen ca. 723 Projekte mit einem geschätzten Kostenvolumen von 8,1 Mrd. € (ausschließlich der Subclusterprojekte).

- Bisher wurden über 800 Projekte mit einem Kostenvolumen von 11,6 Mrd. $€$ abgeschlossen. $17 \%$ der Projekte mussten vorzeitig abgebrochen werden. 
Die unbürokratische Projektabwicklung und die Wirtschaftsnähe begünstigt auch die hohe Beteiligung von KMUs. In den derzeit laufenden Projekten beträgt der KMU-Anteil an allen beteiligten Organisationstypen $42 \%$ und $62 \%$ von allen Industrieteilnehmern. Jährlich werden ca. 200 Projekte neu generiert. Im Vorsitzjahr 2000/2001 2001 konnten 187 neue Projekte mit einem Gesamtvolumen von 450 Mill. $€$ initiiert werden (siehe Abbildung 4-10). Hinzu kommen noch ca. 50 Subclusterprojekte.

\section{Abbildung 4-10: Beteiligung nach Organisationstypen in derzeit laufenden Projekten und neue Projekte pro Jahr}
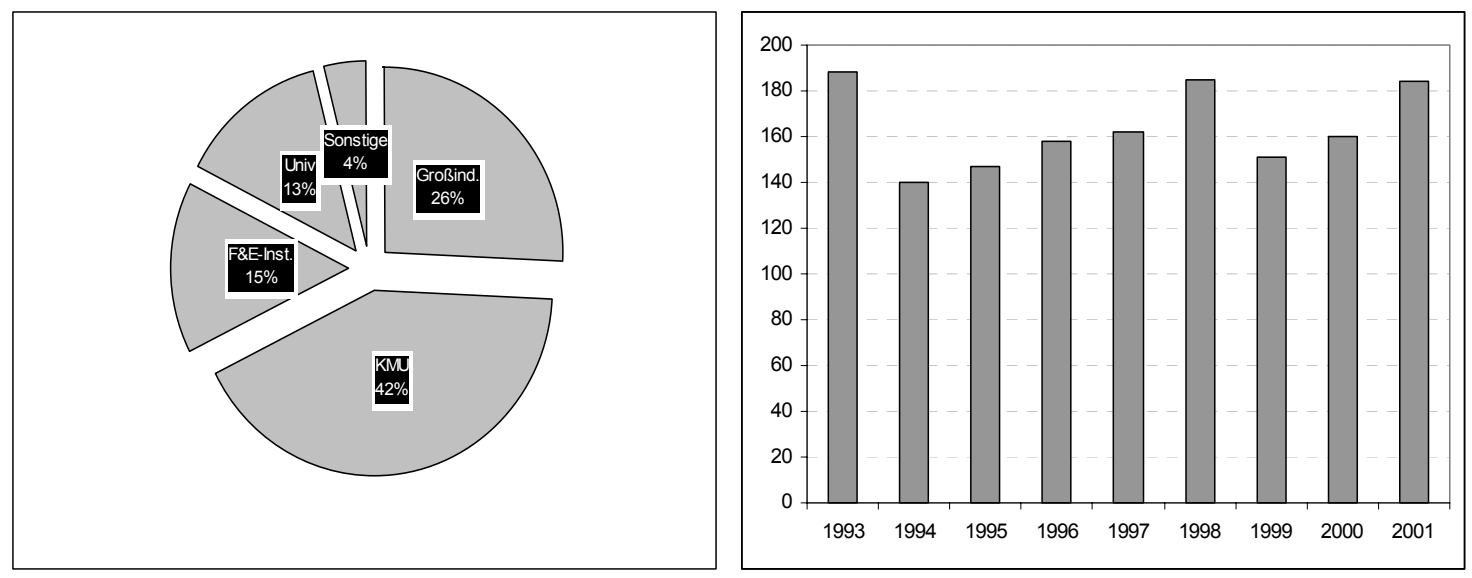

Quelle: EUREKA

Österreichische Partner sind an rund 13\% aller bisher beschlossenen Projekte beteiligt. 250 Projekte wurden bisher mit österreichischen Teilnehmern oder unter österreichischer Führung durchgeführt. Die Kosten dafür betrugen 225 Mio. $€$. Im Jahr 2000/01 kamen 29 neue österreichische Projektbeteiligungen dazu sowie 13 neue Beteiligungen in Schirmprojekten. Österreich ist somit auch in sämtlichen großen Clusterprojekten beteiligt. Sieben Projekte werden insgesamt von österreichischen Teilnehmern koordiniert, darunter auch ein neues Clusterprojekt. Clusterprojekte sind branchenspezifische und von den teilnehmenden europäischen Unternehmen getragene und verwaltete Großprojekte, die zahlreiche Subprojekte mit oft vielen hundert Teilnehmern durchführen. Dementsprechend groß sind auch die Projektvolumina dieser strategischen Clusterprojekte und weisen auch auffallende Ähnlichkeiten mit dem für das 6. RP geplanten neuen Instrument der integrierten Projekte auf. Die Fortführung des renommierten MEDEA Projektes (MEDEA+) beispielsweise verzeichnet ein Projektvolumen von 4000 Mill. $€$ für den Zeitraum 2001-2007. In den seit 2001 laufenden 35 Subprojekten ist Österreich in acht Projekten beteiligt. Sowohl durch die verstärkte Anwendungsorientierung im 6. RP als auch durch den neuen Projekttypus wird es daher zu einer noch stärkeren Überschneidung mit der EUREKA Initiative kommen.

Wie Abbildung 4-11 zeigt, sind insgesamt 68 österreichische Organisationen an laufenden Projekten beteiligt. Dass EUREKA explizit das Ziel einer Einbindung der ost- und mitteleuropäischen Staaten verfolgt hat, zeigt sich auch der mittlerweile festen Verankerung 
einiger Beitrittskandidaten. Die Tschechische Republik liegt mit 83 teilnehmenden Organisationen beispielsweise vor einigen EU-Ländern.

Die starke Anwendungsorientierung von EUREKA zeigt sich auch an den teilnehmenden Organisationstypen in laufenden Projekten. In zwei Drittel aller laufenden Projekte nehmen Industrieunternehmen teil und ein Drittel Forschungsorganisationen. In Österreich liegt der Anteil von Industrieunternehmen bei $62 \%$ und damit knapp unter dem Durchschnitt. Der Anteil von KMU an den Industrieteilnehmern bei laufenden Projekten liegt bei durchschnittlich $60 \%$ ebenfalls ein Indiz für die Attraktivität von EUREKA für kleine und mittlere Betriebe.

Abbildung 4-11:: Zahl der an laufenden Projekten teilnehmenden Organisationen

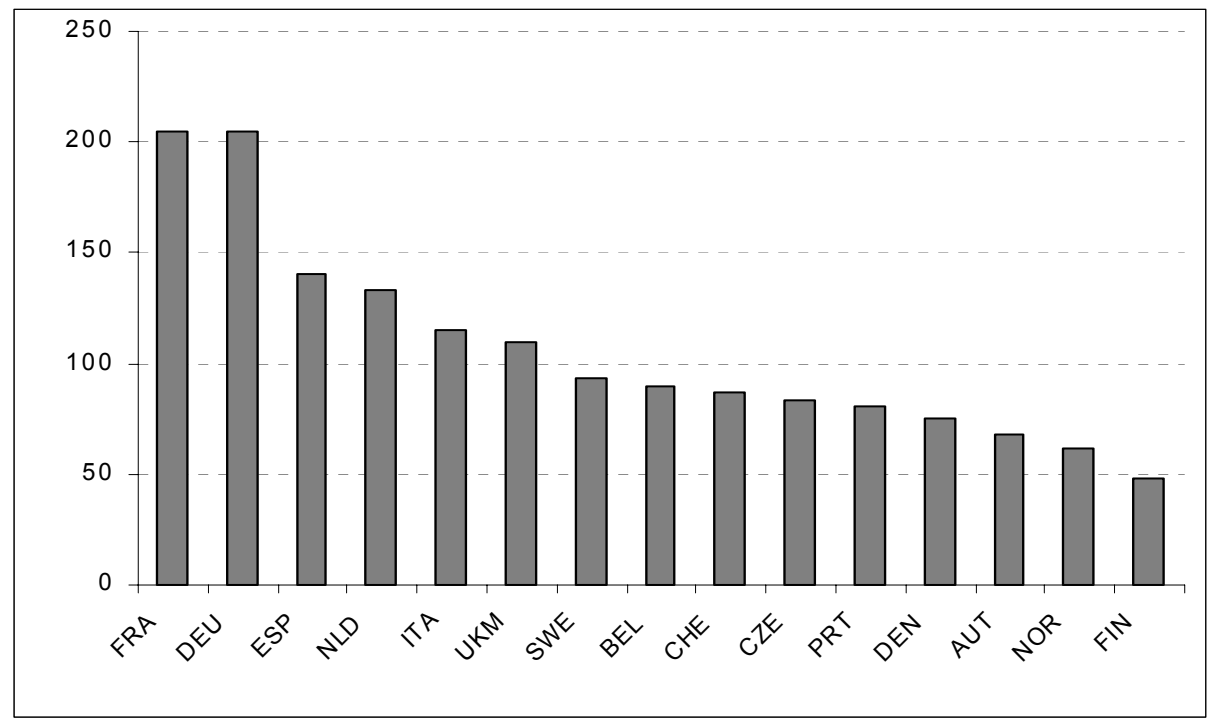

Quelle: EUREKA 
Abbildung 4-12: Zahl der laufenden Projekten nach Organisationstyp

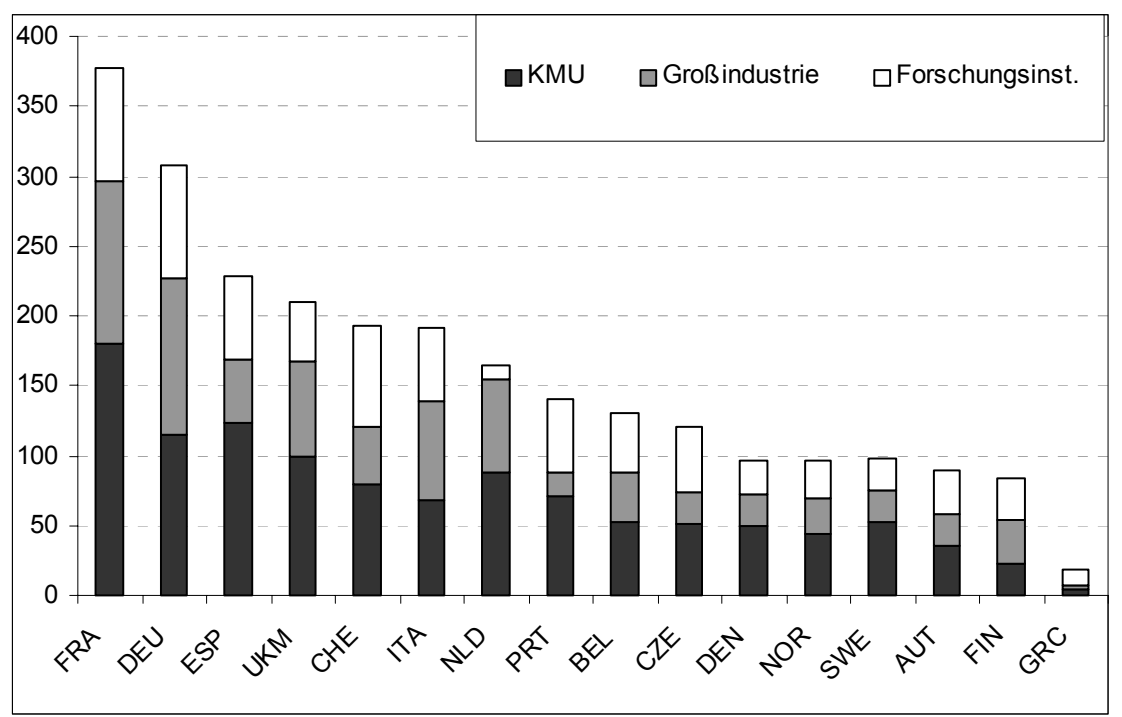

Quelle: EUREKA

In Österreich ist der FFF die wichtigste nationale Förderstelle für Beteiligungen der Wirtschaft an EUREKA-Projekten. Insgesamt wurden im Jahr 200114 EUREKA-Projekte mit einem Barwert von 2,7 Mio. $€$ gefördert. Zusätzliche Förderungen kommen von den Förderstellen der Bundesländer sowie vom FWF (Universitätsbeteiligungen).

\subsection{Die österreichische Beteiligung an COST}

\subsubsection{COST im Überblick}

Österreich ist seit 1971 Mitglied der europäischen Forschungsinitiative COST, die 34 Mitgliedstaaten (15 EU- und 19 Nicht-EU-Mitglieder) sowie Israel umfasst. Gleichzeitig eröffnet COST Kooperationen mit Forschungsinstitutionen aus Drittstaaten wie Russland, Japan, Australien, Kanada und den USA und ist ein wichtiges Forum für die Zusammenarbeit mit dem Donauraum. Ca. 30.000 WissenschaftlerInnen arbeiten in COST als Netzwerk zusammen; 15.000 Benutzer besuchen täglich die COST-Homepage der EK. Besonders wichtig ist COST für Nachwuchswissenschaftler zur Heranführung an internationale Kooperation und kurzfristigen Forscheraustausch im Rahmen der Short Term Scientific Missions. Das im Rahmen von COST aufgebrachte Forschungsvolumen beträgt $€ 2$ Mrd. pro Jahr.

COST fördert wissenschaftlich-technische Forschung im vorwettbewerblichen Bereich auf Grundlage gemeinsamer Koordination, jedoch auf Basis nationaler Finanzierung. Die Projekte umfassen ein Spektrum von der Grundlagenforschung bis zur industriellen Anwendung, insbesondere in multi- und interdisziplinären Bereichen.

Die variable Geometrie der COST-Zusammenarbeit ermöglicht eine Beteiligung der Mitgliedstaaten nach dem „à la carte-Prinzip“: Es besteht freie Wahlmöglichkeit der Beteiligung 
an den einzelnen Forschungsaktionen. Das hohe Interesse der Mitgliedstaaten und die lebendige Wissenschaftskooperation zeigt sich an der hohen Zahl der Forschungsaktionen (dzt. rd. 160, davon 90\% mit österreichischer Beteiligung).

COST stellt eine Infrastruktur zur Verfügung, die es Wissenschaftlern aus ganz Europa ermöglicht, ihre Expertise auszutauschen und miteinander effizient zu kooperieren. Dies ist besonders für junge Forscherteams attraktiv, denen die Flexiblität von COST entscheidende Vorteile bietet: Offenheit für neue Impulse (bottom up approach), Workshops, "Short Term Scientific Missions" und zunehmend weltweite Kooperationen.

\subsubsection{Beteiligung Österreichs an COST}

Die kontinuierlich gestiegene Beteiligung Österreichs an COST ist prozentuell so hoch wie an keinem anderen Forschungsprogramm (vgl. Forschungs- und Technologiebericht 2001) und belegt die lebhafte Kooperation österreichischer Wissenschaftler mit europäischen Partnern, vor allem auf Gebieten der Nanowissenschaften, medizinisch-pharmazeutischer Forschung, Telekommunikation, Materialwissenschaften, Verkehrsforschung, Holzverarbeitung und Papierindustrie. Die erfolgreiche COST-Kooperation bedeutet auch für Österreich eine entscheidende Steigerung des nationalen Innovationspotentials zu günstigen Bedingungen und Zugang zu den gemeinsam erarbeiteten Forschungsergebnissen von gesamteuropäischer Relevanz. Dabei erweist sich COST als besonders wertvolles Instrument für Österreich, für das die Zusammenarbeit mit mittel- und osteuropäischen Staaten besonderes Gewicht hat.

In Österreich gibt es keinen eigenen Budgetansatz für COST-Aktionen, wie es in manchen anderen Mitgliedstaaten der Fall ist (z.B. der Schweiz oder der Tschechischen Republik). Die im Zuge der Teilnahme an COST-Aktionen entstehenden Projektkosten werden durch Eigenmittel der Forschungseinrichtungen getragen, durch Forschungsförderung der Fonds (FWF, FFF), Unternehmen oder im Rahmen der Auftragsforschung.

\subsubsection{COST und das EU-Rahmenprogramm}

Das top-down orientierte Rahmenprogramm und die bottom-up Kooperation COST sind komplementär, wobei COST in seiner flexiblen Arbeitsweise den Europäischen Forschungsraum (ERA) unterstützt. COST hat aufgrund seines großen Erfolges Modellcharakter für neue Kooperationen auch in den Beziehungen Europas zu anderen Erdteilen. Die jüngste Entwicklung hat gezeigt, dass vor allem Kanada, die USA und Australien ihre Beziehungen zu COST intensivieren und auf Aktionsebene verstärkt zum gegenseitigen Nutzen kooperieren. Dabei liefern COST-Projekte wesentliche Initialzündungen zu weiteren Entwicklungen in EUREKA und im Rahmenprogramm, zumal COST-Aktionen vor allem im vorwettbewerblichen Bereich, insbesondere der Grundlagenforschung, angesiedelt sind.

Angesichts der Zielsetzung des künftigen Europäischen Forschungsraums stellt COST ein nahezu ideales Instrument zur Nutzung der vorhandenen nationalen Potentiale auf europäischer Ebene dar. Dies wurde sowohl von dem Generalsekretär und Hohen Vertreter der Europäischen Union, Herrn Solana, als auch dem EU-Forschungskommissar Busquin 
bekräftigt. Beide haben ihr besonderes Interesse am Erfolg von COST und seiner Nutzbarmachung für die Unionspolitiken zum Ausdruck gebracht.

Die COST-Kooperation liegt damit im Interesse der gesamteuropäischen Innovation und dient gleichzeitig der Wahrung der österreichischen Interessen in diesem Prozess, an dem wir konstruktiv teilnehmen wollen.

\section{4 Österreichs Mitgliedschaft in der European Space Agency (ESA)}

Die Europäische Weltraumagentur ESA ist eine selbstständige, multinationale Einrichtung, deren Mitglieder zur gemeinsamen Forschung an wissenschaftlichen und technischen Weltraumvorhaben zusammengeschlossen sind. Zur Zeit sind 15 europäische Staaten Mitglieder der ESA, Österreich ist seit 1987 Vollmitglied.

Das Budget der ESA betrug im Jahr 20012.857 Mio. $€$ (vgl. ESA 2002). Die ESA gliedert ihre Aktivitäten in obligatorische Programme, an denen sich alle Mitglieder beteiligen, und optionale Programme. Die obligatorischen Programme haben vor allem die Aufgabe, die technische und Verwaltungsinfrastruktur bereitzustellen. Weiters enthalten die obligatorischen Programme das Wissenschaftsprogramm, das sich vor allem auf Grundlagenforschung konzentriert. Die Statuten der ESA schreiben eine Beteiligung der Mitgliedsstaaten an diesen verpflichtenden Aktivitäten nach dem Anteil ihrer Wirtschaftskraft, gemessen durch das BIP, vor.

Von ihrem Umfang wesentlich bedeutender als die obligatorischen Programme sind die ESAWahlprogramme. Vor allem das Raumtransportprogramm und die bemannten Raumfahrtaktivitäten, die sich derzeit auf die europäische Beteiligung an der internationalen Raumstation ISS konzentrieren, sind die öffentlich sichtbarsten ESA-Initiativen. Daneben sind satellitengestützte Erdbeobachtung und Telekommunikation zwei weitere wichtige Schwerpunkte in den Wahlprogrammen. Im Gegensatz zu den obligatorischen Programmen steht die Teilnahme den Mitgliedern frei, sodass die Finanzierungsbeiträge pro Land von seinen BIP-Anteilen abweichen können. Die Regeln der ESA für Wahlprogramme sehen weiters vor, dass die geleisteten Beiträge als Zulieferaufträge in das jeweilige Land zurückfließen („Just-Retour-Prinzip“). Damit sind die ESA-Beiträge auch eine Förderung der heimischen Weltraumindustrie, von denen Firmen wie Austrian Aerospace, Siemens, ARC Seibersdorf Research, Joanneum Research oder verschiedene Universitätsinstitute profitieren.

Der österreichische Finanzierungsbeitrag an den Aktivitäten der ESA betrug im Jahr 2001 etwa 25 Mio. €. 13,8 Mio € entfielen davon auf Beiträge für die obligatorischen Programme gemäß dem österreichischen BIP-Anteil von 2,48\%. Darüber hinaus finanziert Österreich verschiedene Wahlprogramme mit insgesamt 14,7 Mio $€$. Die österreichische Beteiligung an den Wahlprogrammen ist damit im Vergleich zu anderen Länder relativ gering und liegt deutlich unter dem BIP-Anteil. Österreichs Engagement konzentriert sich vor allem auf die Bereiche Telekommunikation sowie Erdbeobachtung für Umweltfragen und Meteorologie. Österreich verzichtet unter anderem auf eine Teilnahme an den Aktivitäten zur Errichtung und Nutzung der 
internationalen Raumstation ISS. Die österreichische Beteiligung an Pflicht- und Wahlprogrammen trägt insgesamt etwa $1,2 \%$ des ESA-Gesamtbudgets.

Die ESA sieht in einer engeren Abstimmung und Zusammenarbeit mit der Europäischen Union und dem 6. Rahmenprogramm in luft- und raumfahrtbezogenen Aktivitäten einen Kern der zukünftigen Strategie. Zwei bedeutende Vorhaben in dieser Partnerschaft sind GMES (Global Monitoring for the Enviroment and Security) und GALILEO. GMES soll die europäischen Aktivitäten auf dem Gebiet der satellitengestützten Erdbeobachtung, die bisher durch verschiedene Organisationen durchgeführt wurden, vereinigen.

GALILEO ist eine Initiative zur Errichtung eines europäischen Satellitennavigationssystems. Es soll den Nutzern ermöglichen, jederzeit die Uhrzeit und seinen Standort mit großer Genauigkeit zu ermitteln. Europa macht sich damit von existierenden amerikanischen und russischen Ortungssystemen unabhängig. GALILEO ist damit Grundlage für eine Reihe von zukünftigen Anwendungen in Verkehr, Landwirtschaft, Verteidigung, Rettungsdienste oder Telekommunikation (vgl. Europäische Kommission 2001a). Das System soll ab 2008 zur Verfügung stehen. Die Kosten der Entwicklung und Errichtung werden von der Kommission mit 3,2 Mrd. $€$ veranschlagt. Das ARTIST-Programm ${ }^{6}$ (Austrian Radionavigation Technology and Integrated Satnav Services and Products Testbed) Das Programm wird als österreichisches Testbed für Anwendungen, die auf GALILEO aufbauen sollen, eingerichtet. Das Programm wird vom BMVIT mit insgesamt 2 Mill. Euro finanziert und von der Austrian Space Agency durchgeführt.

\section{Abbildung 4-13: Österreichs Finanzierungsbeitrag an der ESA, 2001}

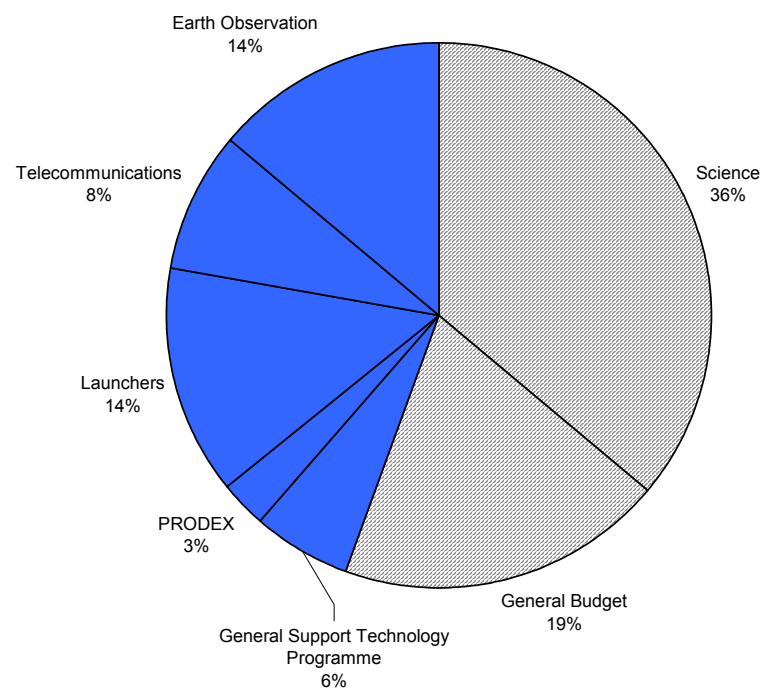

Quelle: Austrian Space Agency

${ }^{6}$ http://www.galileo-austria.at/Artist/index.htm 


\section{Technologieförderung in Österreich}

Die direkte und die steuerliche Förderung von Forschung und Entwicklung hat jeweils ihre spezifischen Vor- und Nachteile (OECD, 1998). Ein den jeweiligen Bedingungen angepasster Mix kann die Effektivität des Förderungssystems erhöhen. Die Mehrzahl der OECD-Länder unterstützen heute F\&E sowohl mittels direkter Förderungen als auch zunehmend durch steuerliche Anreize. Die Effektivität eines Instruments der F\&E-Förderung hängt auch von der Intensität des Einsatzes der anderen Komponenten des F\&E-Förderungssystems ab. Neuere Forschungsergebnisse weisen darauf hin, dass sich die direkte und die steuerliche F\&EFörderung substitutiv zueinander verhalten (Guellec - van Pottelsberghe, 2000 bzw. OECD, 2000): Eine Erhöhung der Intensität des einen Instruments vermindert die Wirkung des anderen. Daraus ergibt sich ein Bedarf, die Förderungsinstrumente aufeinander abzustimmen.

\subsection{Direkte Technologieförderung}

Für die Bewertung der direkten Technologieförderung des Bundes werden zwei unterschiedliche Datenquellen benutzt: In Kapitel 6.1.1 wird die Entwicklung der direkten Technologieförderung anhand der Förderungsdatenbank FINKORD des Bundeskanzleramts vorgenommen. Damit ist es möglich, die Technologieförderung im Zeitablauf und in Relation zu anderen Förderschwerpunkten darzustellen. In Kapitel 6.1.2 werden die Schwerpunkte der Technologieförderungen basierend auf einer rezenten Erhebung von Joanneum und WIFO (Leo et al., 2002) herausgearbeitet. Letztere Arbeit hat im Unterschied zu FINKORD jedoch eine weitere Definition der direkten Technologieförderung gewählt. In der FINKORD-Datenbank wird definitionsgemäß die direkte unternehmensbezogene Wirtschaftsförderung des Bundes dokumentiert. Dies bedeutet, dass etwa die Förderungslinien des FWF, der Jubiläumsfonds der Nationalbank und die Ressortforschungsprogramme von BMBWK und BMWA nicht einbezogen sind. Darüber hinaus werden die Förderungen der Bürges getrennt ausgewiesen. Durch die unterschiedliche Abgrenzung der direkten Technologieförderung ist der Barwert der erfassten Programme in der Joanneum - WIFO Studie deutlich höher.

\subsubsection{Die direkte unternehmensbezogene Technologieförderung des Bundes}

Die Förderungsdatenbank FINKORD des Bundeskanzleramts enthält umfangreiche Informationen über die direkte unternehmensbezogene Wirtschaftsförderung des Bundes. Sie ermöglicht unter u. a. Auswertungen nach Förderungsschwerpunkten, darunter die Technologieförderung. Die Zuordnung zu den Förderungsschwerpunkten erfolgt nach den Förderungsinstitutionen bzw. den Programmlinien dieser Einrichtungen.

Tabelle 6.1 bietet einen Überblick über die Entwicklung der direkten Technologieförderung des Bundes im Zeitraum 1997 - 2001, wobei die Angaben für 2001 noch als vorläufig zu betrachten sind. Gemessen am Barwert der Förderung erreichte die Technologieförderung - nach einem kräftigen Anstieg im Vorjahr - im Jahr 2000 mit 185 Mio. € einen vorläufigen Höhepunkt. Damit liegt das Förderungsvolumen um rund 35\% über dem Niveau der Jahre 1997/1998. Die geförderten Investitionen erreichten mit 1,2 Mrd. € ebenfalls einen Höchstwert. 
Tabelle 5-1: Direkte unternehmensbezogene Technologieförderung, 1997 - 2001

$\begin{array}{lrrrrr} & \mathbf{1 9 9 7} & \mathbf{1 9 9 8} & \mathbf{1 9 9 9} & \mathbf{2 0 0 0} & \mathbf{2 0 0 1} \\ & & & & & 807 \\ \text { Anzahl Unternehmen } & 769 & 787 & 867 & 832 & 1.046 \\ \text { Anzahl Projekte } & 1.065 & 1.041 & 1.140 & 1.095 & \\ & & & & & \\ \text { Investitionsvolumen in Mio. } € & 1.050 & 1.182 & 1.076 & 1.204 & 979 \\ \text { Förderungsvolumen in Mio. } € & 367 & 420 & 420 & 438 & 345 \\ \text { Barwert in Mio. } € & 136 & 137 & 179 & 185 & 148\end{array}$

Quelle: FINKORD

Abbildung 5-1 illustriert die Entwicklung des Barwerts der Technologieförderung in Relation zu anderen Förderungsschwerpunkten (Regionalförderung, Umweltförderung, Sonstige Förderungen bestehend aus Allgemeiner Investitionsförderung, nicht-industriell-gewerblicher Förderung und Bürges). Die Technologieförderung hat im Beobachtungszeitraum (abgesehen von den noch vorläufigen Werten für 2001) eine stetige Aufwärtsentwicklung zu verzeichnen. Beim zweiten großen Förderungsschwerpunkt, der Regionalförderung, ist nach dem Ende der letzten Programmplanungsperiode (interimistisch) ein drastischer Rückgang eingetreten.

\section{Abbildung 5-1: Barwert der unternehmensbezogenen Wirtschaftsförderung des Bundes} nach Förderarten (in Mio. $€$ )

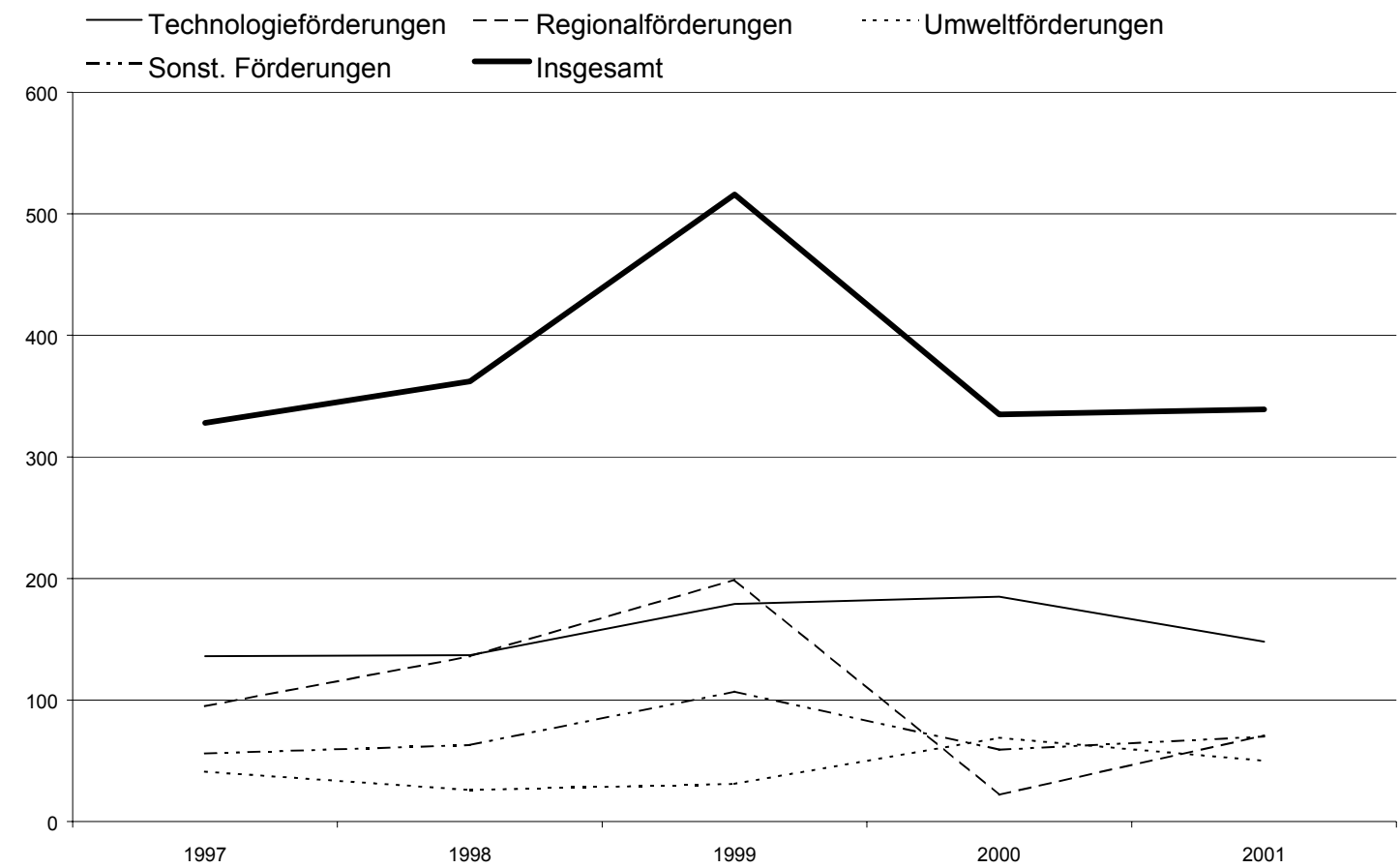

Quelle: FINKORD 
Primär bedingt durch den Rückgang der Regionalförderung überschritt der Anteil der Technologieförderung am gesamten Barwert der direkten unternehmensbezogenen Wirtschaftsförderung des Bundes im Jahr 2000 erstmals die 50\%-Marke (Abbildung 5-2). Aber auch abgesehen von diesem außerordentlichen Wert etabliert sich die Technologieförderung in mehrjähriger Betrachtung als quantitativ bedeutendstes Segment der direkten Wirtschaftsförderung des Bundes.

\section{Abbildung 6.2: Barwert der unternehmensbezogenen Wirtschaftsförderung des Bundes nach Förderarten (Anteil in \%)}

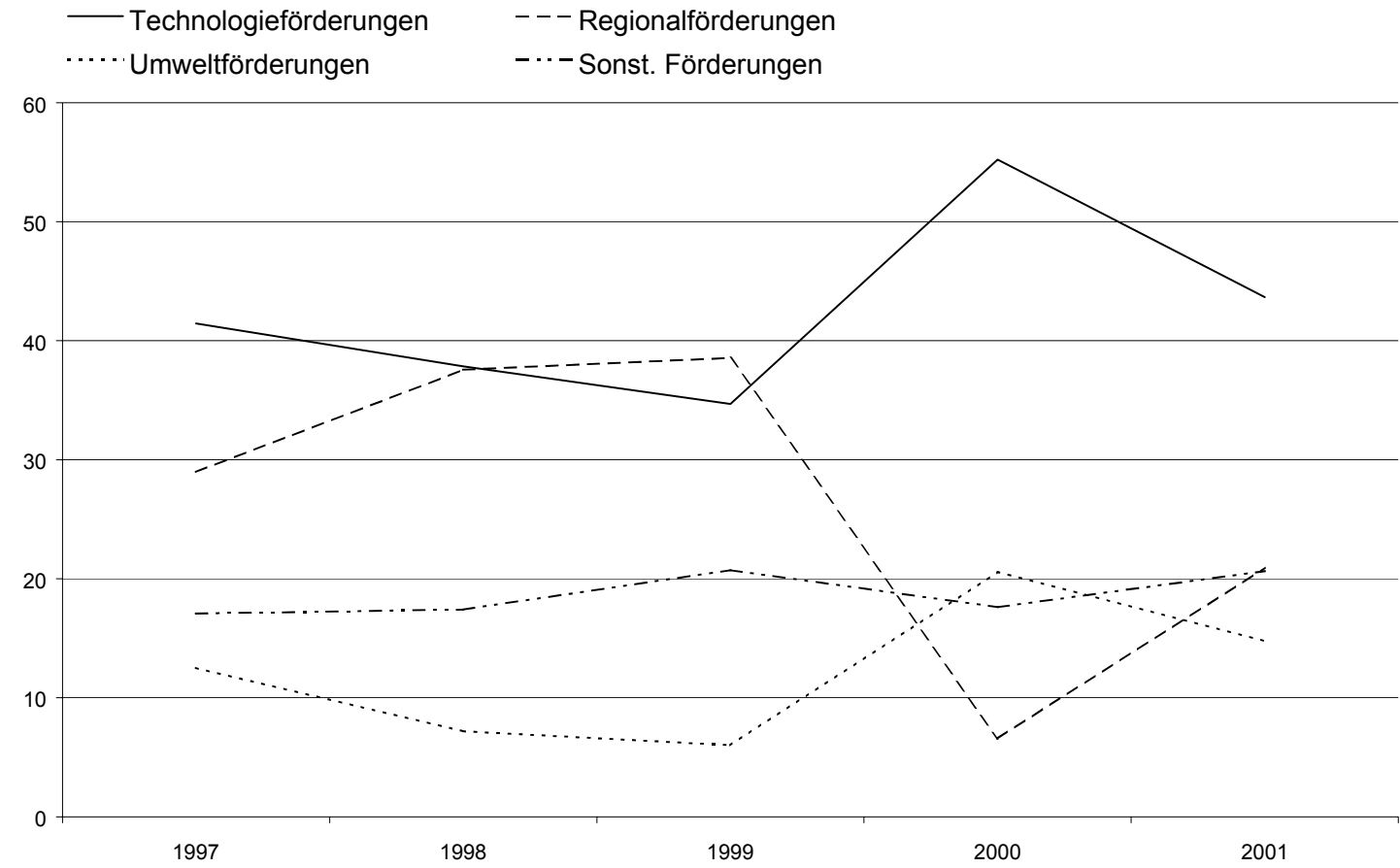

Quelle: FINKORD

\subsubsection{Schwerpunkte der direkten Technologieförderung des Bundes}

In Pilotstudie von Joanneum Research und WIFO (Leo et al., 2002) wurden die laufenden, direkten Technologiefördermaßnahmen des Bundes erhoben. Dabei wurde versucht, die faktischen Schwerpunkte der direkten Technologieförderung zu erheben, zu klassifizieren und vergleichbar darzustellen. Mittels einer Erhebung bei österreichischen Förderinstitutionen wurden die Ziele, die Zielgruppen, die Monitoring- und Evaluierungskultur und die Abwicklung der Maßnahmen erhoben.

Die Entwicklung der Förderbarwerte der untersuchten Fördermaßnahmen zeigt in den letzten Jahren eine kontinuierliche Steigerung und erreichte im Jahr 2000329 Mio. $€$. Die Förderbarwerte haben sich in den erfassten Programmen seit 1997 um rund 97 Mio. $€$ erhöht. Mit rund 215 Mio. $€$ Förderbarwert hat das BMVIT das bei weitem größte Fördervolumen. Auf 
das BMWA entfallen rund 83 Mio. $€$, auf das BMBWK 16,5 Mio. $€$ und 13,4 Mio. $€$ auf andere Förderinstitutionen.

Insgesamt wurden 73 Maßnahmen als direkte Technologieförderungen identifiziert und ausgewertet $^{7}$. Die Fördermaßnahmen werden in drei Kategorien unterteilt. Während die Förderprogramme inhaltlich spezifizierte Förderaktivitäten darstellen, zielen unspezifische direkte F\&E-Förderungen inhaltlich auf nicht spezifizierte Aktivitäten. Die dritte Kategorie stellt die institutionelle Förderung dar, in der Institutionen direkt unterstützt werden.

Technologieförderung hat sich in mehrjähriger Betrachtung als quantitativ bedeutendstes Segment der direkten Wirtschaftsförderung des Bundes etabliert. Dabei dient die Mehrzahl der Fördermaßnahmen der Verbesserung der Verbindung zwischen Forschung und Innovation.

Wie Tabelle 5-2 zeigt, gibt die Unterteilung der direkten Technologieförderung in diese Kategorien ein unterschiedliches Bild, je nach dem ob die Zahl der Fördermaßnahmen oder die Förderbarwerte herangezogen werden.

Tabelle 6.2: Zahl der Maßnahmen nach Förderkategorie und Förderbarwerte ${ }^{8}, 2000$

\begin{tabular}{lcc}
\hline Förderungsart & $\begin{array}{c}\text { Anzahl der } \\
\text { Fördermaßnahmen }\end{array}$ & $\begin{array}{c}\text { Förderbarwerte im Jahr 2000 } \\
\text { (in Mio. ATS) }\end{array}$ \\
\hline Programmförderung & 18 & 642 \\
unspezifische direkte F\&E Förderung & 40 & 3.650 \\
institutionelle Förderung & 15 & 240 \\
\hline Gesamt & 73 & 4.532 \\
\hline
\end{tabular}

Quelle: Leo et al. (2002)

Mehr als die Hälfte der Maßnahmen sind der Kategorie unspezifische Technologieförderung zuzurechnen (40 Maßnahmen), durch die verschiedene Dimensionen des Innovationsprozesses unterstützt werden. Etwa gleich viele Programme gibt es bei der Programmförderung (18 Programme) und bei institutionellen Förderungen (15 Programme). Dieses Bild wird durch die Verteilung der Förderbarwerte bestätigt: rund $80 \%$ der Förderbarwerte werden über unspezifische direkte F\&E-Förderungen vergeben, rund 15\% über Programmförderungen und $5 \%$ über institutionelle Förderungen.

Einschränkend ist anzumerken, dass die erfassten Fördermaßnahmen nicht die Gesamtheit der direkten Technologiefördermaßnahmen des Bundes abbilden, da die Förderprogramme des Bundesministeriums für Verkehr, Innovation und Technologie (BMVIT) nur zum Teil enthalten sind. Fördermaßnahmen des BMVIT, die über Programmträger abgewickelt werden, sind erfasst; Aktivitäten, die das Ministerium selbst durchführt (insbesondere die Fördermaßnahmen MOVE, Impulsprogramm Biomedizinische Technik, Aeronautics Programme - take off und Pilotprogramm Donau) konnten jedoch nicht berücksichtigt werden.

8 Die Summe der Maßnahmen (60) von denen Förderbarwerte angegebenen werden können, unterscheidet sich aus erhebungstechnischen Gründen von der Gesamtzahl der erfassten Förderaktivitäten (73), vgl. Leo et al. 2002 


\section{Abbildung 5-3: Anzahl der Fördermaßnahmen und Förderbarwerte nach Trägern}

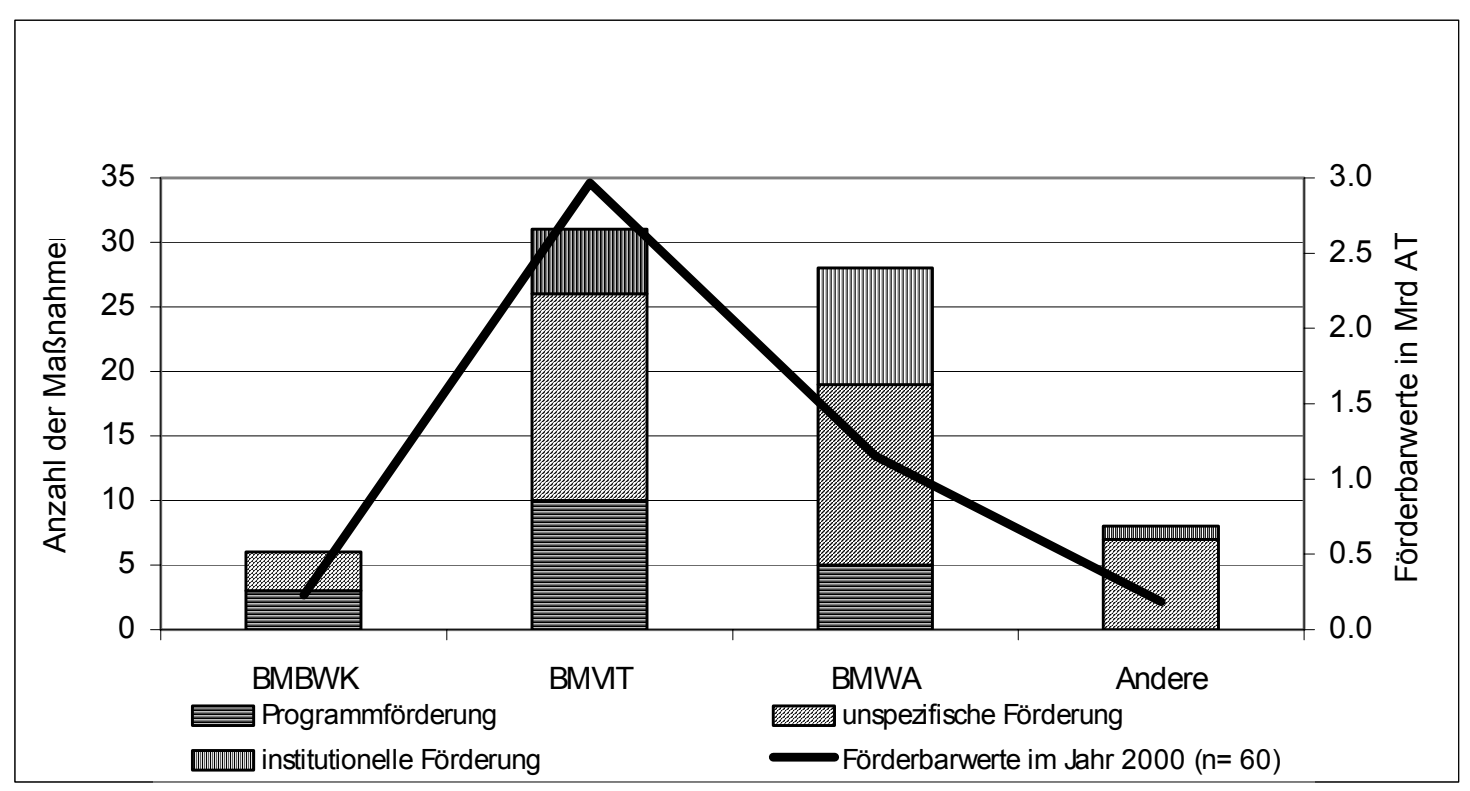

Quelle: Leo et al. (2002).

Die Verteilung der Mittel spiegelt zum einen die große Bedeutung einer breiten und kaum spezifizierten direkten Innovationsförderung wider. Zum anderen zeigt sich, dass die Versuche stärker Schwerpunkte zu bilden, vor allem in der Zahl der Programme und weniger im Umfang der bereitgestellten Mittel sichtbar werden.

Abbildung 5-3 zeigt die Förderbarwerte nach Ministerien in Relation zu der Anzahl der Fördermaßnahmen im Jahr 2000. Auffällig ist dabei die große Anzahl von Programmen im Verhältnis zum relativ geringen Förderbarwert beim BMWA, dem BMBWK und den anderen Trägerinstitutionen in der Technologieförderung. Die relativ geringen Förderbarwerte in Relation zur Anzahl der Förderungen bei diesen Institutionen stehen in starkem Kontrast zum BMVIT. Dieses weist sowohl eine große Anzahl von Initiativen als auch den höchsten Förderbarwert aus. Dies ist nicht zuletzt auf den Umstand zurückzuführen, dass sich im Einflussbereich des BMVIT die großen Förderungsfonds befinden.

Welche Schwerpunkte der direkten Technologieförderung in Österreich gemäß einer Einteilung des Europäischen Aktionsplanes für Innovation (vgl. Europäische Kommission 1997) verfolgt werden, zeigt Abbildung 5-4. Bei der Interpretation der zugeordneten Förderbarwerte muss berücksichtigt werden, dass diese je zur Hälfte zugerechnet wurden, wenn zwei Schwerpunkte angegeben wurden. 


\section{Abbildung 5-4: Förderaktivitäten nach Förderschwerpunkt}

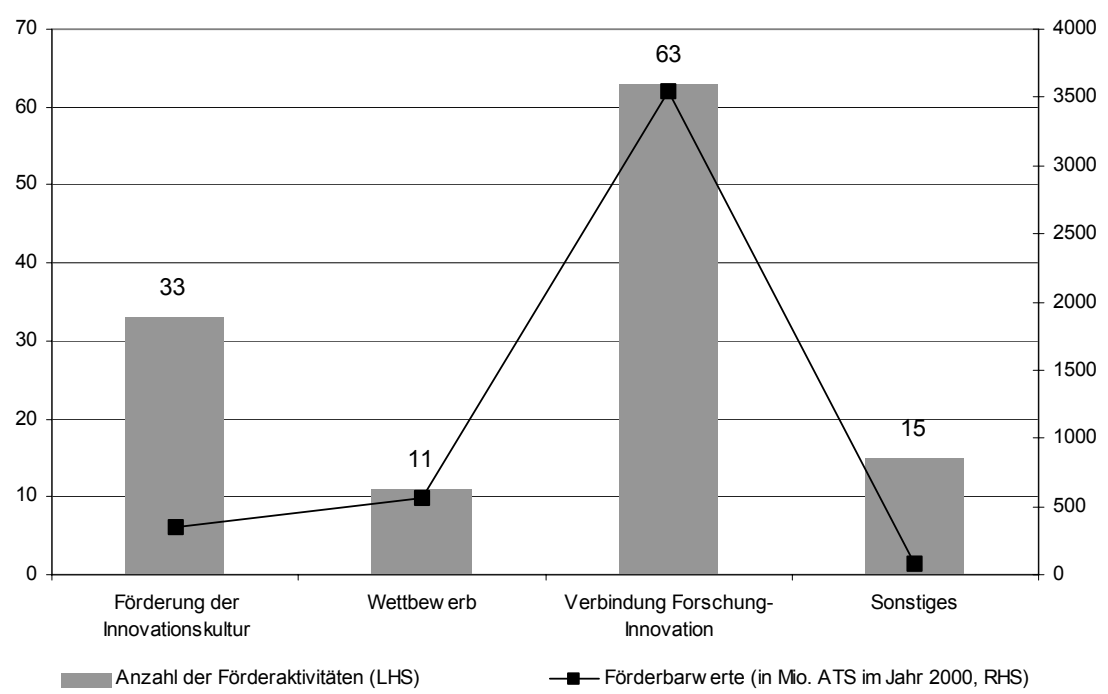

Quelle: Leo et al. (2002).

Die jeweiligen Aktionslinien lassen sich in drei Hauptgruppen unterteilen, die wiederum unterschiedliche Förderbereiche beinhalten:

- Die erste Aktionslinie Förderung der Innovationskultur umfasst Aus- und Weiterbildung, Mobilität von Studenten, Forschungs- und Lehrpersonal, Bewusstseinsbildung in der Öffentlichkeit und Einbindung von Betroffenen, Innovation und Unternehmensführung, Behörden, Förderung von Clusterbildung und Kooperationen zum Zwecke der Innovation.

- Die zweite Aktionslinie bezieht sich auf die Schaffung eines günstigen rechtlichen, ordnungspolitischen und finanziellen Umfelds und spricht Wettbewerbsaspekte und technologische Wettbewerbsfähigkeit an. Die Unterpunkte sind der Schutz geistigen Eigentums, die Vereinfachung von Verwaltungsverfahren, das gesetzliche und regulatorische Umfeld, die Finanzierung von Innovationsprojekten und steuerliche Aspekte.

- Die dritte Aktionslinie, die der besseren Abstimmung von Forschung und Innovation dient, umfasst Strategien zu Forschung und Entwicklung, Stärkung der Unternehmensforschung, Gründung von technologiebasierten Unternehmen, verstärkte Kooperation zwischen Forschung, Universitäten und Unternehmen, Stärkung der Technologie- und Know-howAufnahme von KMUs.

Abbildung 5-4 macht deutlich, dass der Fokus der Fördermaßnahmen vor allem auf die Verbesserung der Verbindung zwischen Forschung und Innovation liegt. Diese Aktionslinie wurde 63mal genannt. Die Förderung der Innovationskultur liegt mit 33 Nennungen an zweiter Stelle. Deutlich seltener sind Aspekte des Wettbewerbs explizites Ziel der Maßnahmen (11 
Nennungen). Sonstige Förderziele wurden in 15 Fällen genannt. ${ }^{9}$ Hinsichtlich der finanziellen Volumina zeigt sich ebenfalls eine deutliche Konzentration auf die Stärkung der Verbindung zwischen Forschung und Innovation. Trotz der häufigen Nennungen der Förderung der Innovationskultur als Ziel der Maßnahmen sind nur vergleichsweise geringe finanzielle Mittel dieser Aktionslinie gewidmet. Die Differenz zwischen häufiger Nennung und tatsächlicher finanzieller Förderung ergibt sich daraus, dass vornehmlich Maßnahmen mit geringen Fördermitteln diesem Ziel gewidmet sind.

Weitere Eigenheiten der direkten Technologieförderung in Österreich lassen sich wie folgt charakterisieren:

- Die meisten Fördermaßnahmen verfolgen mehrere Ziele gleichzeitig. Während etwas mehr als die Hälfte Fördermaßnahmen Produkt- und Prozessinnovationen unterstützt, wird Grundlagenforschung und vorwettbewerbliche Forschung von $48 \%$ bzw. $44 \%$ der Förderungen forciert. Technologietransfer ist ein weiterer Schwerpunkt der direkten Förderprogramme: 41\% der Förderungen sind diesem Ziel gewidmet.

- Hinsichtlich der geförderten Aktivitäten weisen die Institutionen unterschiedliche Förderungsprofile auf. Während einige nur sehr spezifische Aktivitäten fördern (z. B. fördert der FWF fast ausschließlich Grundlagenforschung), weisen andere eher breite Förderaktivitäten auf.

- Das klassische Instrument der „verlorenen Zuschüsse“ ist nach wie vor das wichtigste Mittel direkter Technologieförderung in Österreich. Insgesamt wurden in $62 \%$ der 76 Programme mittels Direktzuschüssen gefördert. Dieses Förderinstrument liegt damit in seiner Bedeutung klar vor anderen monetären Unterstützungen wie geförderte Kredite, Zinszuschüsse und Haftungsübernahmen. Die österreichische Technologieförderung weist allerdings hinsichtlich der Instrumente durchaus innovative Elemente auf. Dem in der Technologie- und Innovationsforschung immer wieder konstatierten Befund fehlender Information und Awareness über technologische Entwicklungen und Innovationspotentiale wird in unterschiedlichen Programmen entgegenzuwirken versucht. So verstehen sich $22 \%$ der Programme als Initiativen zur Informationsweitergabe/Öffentlichkeitsarbeit, in 11\% der Programme werden Beratungskosten übernommen.

- Die Kultur des begleitenden Monitorings ist in Österreich relativ stark ausgeprägt. $67 \%$ der Programme begleiten alle geförderten Projekte mittels Monitoring, rund $8 \%$ zum Teil. Bei einem Viertel der Programme ist kein Monitoring vorgesehen.

9 Die Summe der Nennungen übersteigt die Summe der Maßnahmen (73), weil zur Beantwortung der Frage bis maximal zwei Nennungen zulässig waren. Insgesamt wurden die möglichen Förderlinien 122 mal angegeben, wodurch es zu Doppelzählungen in den jeweiligen Aktionslinien kommt. Die angegebenen Werte können daher nur ungefähr Auskunft über die Schwerpunkte der direkten Technologieförderung geben. Für die Zahl der Förderbarwerte gilt Analoges wie in Fußnote 9. Die angegebenen Barwerte geben somit nur jene Förderaktivitäten wieder, über welche für das Jahr 2000 Werte verfügbar sind. Auch die Förderbarwerte beinhalten Doppelzählungen und geben damit nur ungefähre Auskunft über die finanziellen Förderungen in den jeweiligen Aktionslinien. 
- Deutlich weniger stark ausgeprägt ist die Evaluierungskultur der Förderprogramme: Nur etwa $25 \%$ der laufenden Programme wurden bisher einer Evaluierung unterzogen ${ }^{10}$.

- Etwas mehr als $60 \%$ der Fördermaßnahmen werden mittels Anträgen der Förderwerber (ohne vorhergehender Ausschreibung) abgewickelt. Die auch international immer bedeutsamer werdende Verfahrensart der Ausschreibungen/Wettbewerbe findet auch in Österreich relativ weite Verbreitung. Immerhin 31\% der Programme (z. B. alle Programme der TIG, Impulsprogramm Biotechnologie, Impulsprogramm Fachhochschulen-Wirtschaft) bedienen sich dieses Verfahrens.

- Trotz einer "groben" Aufgabenteilung der einzelnen Förderinstitutionen, kommt es in vielen Fällen zu Überschneidungen der Fördermaßnahmen, insbesondere dort, wo ähnliche Förderziele angesprochen werden. Beispielsweise werden Programme zur Verstärkung der Kooperation zwischen Forschung, Universitäten und Unternehmen von acht verschiedenen Förderorganisationen durchgeführt. Mögliche Überschneidungen bzw. die Bündelung der Förderprogramme wären zu untersuchen.

- Damit zusammenhängend ist das Förderprofil der einzelnen Institutionen sehr unterschiedlich ausgeprägt. Die große Anzahl von Maßnahmen macht die Förderlandschaft - insbesondere für Klein- und Mittelbetriebe - unübersichtlich. Zudem gibt es zwischen dem BMWA und dem BMVIT großflächige Überschneidungen bei der Förderung von vorwettbewerblicher Forschung und von Produkt- und Prozessinnovationen. Beide Bereiche zusammen stellen den Kernbereich von Innovationsaktivitäten im Unternehmenssektor dar.

\subsection{Steuerliche F\&E-Förderung ${ }^{11}$}

Die Mehrzahl der OECD-Länder unterstützt heute F\&E sowohl mittels direkter Förderungen als auch zunehmend durch steuerliche Anreize. Letztere wurden überwiegend erst seit Anfang der achtziger Jahre eingeführt. In jüngster Zeit hat ihre Verbreitung deutlich zugenommen. Nach einer von der OECD (2001d) zusammengestellten Übersicht verfügen bereits 17 OECD-Länder über steuerliche Anreize für F\&E. Die EU widmet steuerlichen Instrumenten der F\&E-Förderung in jüngster Zeit erhöhte Aufmerksamkeit. Dabei geht es um die Erhöhung der Effektivität und Kohärenz der steuerlichen Anreize für F\&E, insbesondere durch die Verbreitung von "Good Practices", sowie um deren koordinierten Einsatz zur Bewältigung gemeinsamer Herausforderungen im Bereich F\&E und Innovation ${ }^{12}$ ).

Eine effizient gestaltete steuerliche Förderung von F\&E kann die F\&E-Investitionen der heimischen Unternehmen budgetschonend stimulieren, und damit zur Erreichung des Zieles der Bundesregierung beitragen, die Ausgaben für Forschung und Entwicklung in Österreich bis zum Jahr 2005 auf 2,5\% des BIP zu heben.

10 Einschränkend ist jedoch anzumerken, dass bei einigen laufenden bisher nicht evaluierten Maßnahmen sehr wohl Zwischen- und/oder Endevaluierungen vorgesehen sind.

11 Nähere Details bei Hutschenreiter - Aiginger (2001) und Hutschenreiter (2002).

12 Detailliertere Informationen über die Praxis in den EU-Ländern bietet Europäische Kommission (1999, 1999a, 2002). 


\subsubsection{Der Forschungsfreibetrag}

Das weitaus bedeutendste Instrument der steuerlichen F\&E-Förderung in Österreich ist der Forschungsfreibetrag (FFB).

Dieser ist im Einkommensteuergesetz geregelt. Gemäß § 4 Abs. 4 EStG steht - zusätzlich zu der sofortigen Geltendmachung als Betriebsausgaben - für "Aufwendungen zur Entwicklung oder Verbesserung volkswirtschaftlich wertvoller Erfindungen" ein FFB zu. Davon ausgenommen sind "Verwaltungs- und Vertriebskosten sowie Aufwendungen für Wirtschaftsgüter des Anlagevermögens". Weiters heißt es im EStG: "Der volkswirtschaftliche Wert der angestrebten oder abgeschlossenen Erfindung ist durch eine Bescheinigung des Bundesministers für wirtschaftliche Angelegenheiten nachzuweisen. Die Bescheinigung ist nicht erforderlich, wenn die Erfindung bereits patentrechtlich geschützt ist."

Mit der Steuerreform 2000 wurde der FFB neu gestaltet. Dabei wurde u. a.

- der FFB auf generell 25\% der F\&E-Ausgaben angehoben und

- für "zusätzliche" (den Durchschnitt der letzten drei Jahre überschreitende) F\&E-Ausgaben ein FFB von $35 \%$ eingeführt.

Obwohl die steuerlichen Anreize für F\&E in Österreich vergleichsweise großzügig waren, wurden auch Schwachpunkte des FFB festgestellt (siehe Hutschenreiter - Aiginger, 2001).

- Keine besondere Begünstigung von KMUs. Im Unterschied zu anderen OECD-Ländern sieht Österreich mit dem FFB keine besondere Begünstigung von kleinen und mittleren Unternehmen vor.

- Gewinnabhängigkeit des Forschungsfreibetrags. Die Inanspruchnahme des FFB ist vom Vorhandensein hinreichend hoher Gewinne abhängig. Gewinnabhängige Anreize benachteiligen u. a. technologieorientierte Unternehmensneugründungen, die in der Startphase in der Regel keine Gewinne erzielen. Darüber hinaus wirkt der FFB in der Tendenz prozyklisch.

- Ineffektive Differenzierung des Freibetragssatzes. Aus der Einführung eines besonderen Anreizes für zusätzliche F\&E-Ausgaben (FFB von generell $25 \%$ und $35 \%$ für zusätzliche F\&E-Ausgaben) im Rahmen der Steuerreform 2000 war die Absicht erkennbar, neu F\&E betreibende Unternehmen wie etwa forschungsintensive Start-up-Unternehmen besonders zu begünstigen. Die Verwendung eines gleitenden Dreijahresdurchschnitts zur Ermittlung der "zusätzlichen" F\&E-Ausgaben bewirkt jedoch, dass die effektive Mehrbegünstigung dieser zusätzlichen F\&E-Ausgaben sehr gering ist.

- "Volkswirtschaftlich wertvolle Erfindungen". Eine im vorliegenden Zusammenhang sinnvolle, wohlfahrtsökonomische Definition des "volkswirtschaftlichen Werts" einer Erfindung ("soziale Erträge") eignet sich nicht als Konzept der Verwaltungspraxis. Eine Vorabbewertung des F\&E-Outputs übersteigt die Möglichkeiten der Administration bei weitem. Hingegen gibt es aus volkswirtschaftlicher Sicht gute Argumente dafür, dass unternehmerische F\&E-Ausgaben aufgrund der damit verbundenen positiven externen Effekte generell förderungswürdig sind. Es genügt daher zu überprüfen, ob es sich bei den geltend gemachten Ausgaben tatsächlich um F\&E-Ausgaben handelt. Die unterstellte 
Äquivalenz von "volkswirtschaftlichem Wert" und dem Vorliegen eines patentrechtlichen Schutzes ist aus heutiger Sicht ebenfalls anfechtbar.

Weiters wurde darauf hingewiesen, dass seit der Abschaffung des Investitionsfreibetrags unternehmerische Investitionen in die F\&E-Infrastruktur (wie andere Investitionen auch) schlechter gestellt sind als zuvor.

\subsubsection{Die Reform 2002}

Die im Rahmen des "Konjunkturgipfels" der Bundesregierung im Dezember 2001 vorgestellte Neugestaltung der steuerlichen F\&E-Förderung (Österreichische Bundesregierung, 2001) sieht eine Beseitigung einiger Schwächen der bisherigen steuerlichen F\&E-Förderung vor. Die mit der Verabschiedung des_Konjunkturbelebungsgesetzes (Bundesministerium für Finanzen, 2002a) im März 2002 verabschiedete Reform umfasst die folgenden Eckpunkte:

1. Der FFB für Aufwendungen für "volkswirtschaftlich wertvolle Erfindungen" (25\% bzw. $35 \%)$ bleibt voll erhalten.

2. Wahlweise zur bisherigen Regelung 1. kann der Unternehmer einen FFB von $10 \%$ für alle weiteren Forschungsausgaben laut OECD-Definition in Anspruch nehmen.

3. Wahlweise zu 1. und 2. wird eine Forschungsprämie von 3\% für Forschungsausgaben laut OECD-Definition eingeführt.

Der "neue FFB" gemäß Punkt 2. beruht auf einem - gegenüber der bisher vorwiegend am Erfindungsbegriff orientierten Abgrenzung - weiter gefassten Begriff von Forschungsaufwendungen auf Grundlage des "Frascati Manual" der OECD (1994). Gemäß den Erläuterungen (Bundesministerium für Finanzen, 2002, S. 3) kann der neue FFB auch 'parallel' zum bisherigen FFB in Anspruch genommen werden. Dabei soll die gleichzeitige (zusätzliche) Geltendmachung für ein und dieselbe Aufwendung ausgeschlossen sein. Es wird demnach die Möglichkeit bestehen, den Forschungsfreibetrag von Aufwendungen nach der (engeren) bisherigen Definition in Höhe von $25 \%$ bzw. $35 \%$ in Anspruch zu nehmen und zudem für die F\&E-Aufwendungen nach der neuen Definition, soweit sie darüber hinaus gehen, den $10 \%$ Forschungsfreibetrag zu beanspruchen.

Ähnlich wird ausgeschlossen, dass die Forschungsprämie für dieselben Aufwendungen geltend gemacht wird wie der bisherige FFB. Die gleichzeitige Inanspruchnahme des neuen FFB (10\%) und der Forschungsprämie hingegen ist strikt ausgeschlossen: "Wird in einem Jahr ein neuer Forschungsfreibetrag bei einem Forschungsprojekt in Anspruch genommen, ist die Forschungsprämie in diesem Jahr generell (also auch hinsichtlich anderer Forschungsprojekte) ausgeschlossen" (Bundesministerium für Finanzen, 2002, S. 6).

Im Licht der festgestellten Schwachpunkte des bisherigen FFB ist die Einführung einer Forschungsprämie, also eines gewinnunabhängigen Anreizes für F\&E, positiv zu bewerten. Es ist dies eine Maßnahme zur Verstetigung der F\&E-Aktivitäten und eine deutliche Verbesserung für Start-up-Unternehmen, die in der Anfangsphase oft keine Gewinne erzielen. Ebenfalls positiv zu bewerten ist der Übergang zu einer internationalen Standards entsprechenden Definition der F\&E-Ausgaben für den neuen FFB und für die Forschungsprämie. Aufgrund der 
Beibehaltung des bisherigen FFB kommen jetzt - international einzigartig - zwei Definitionen von F\&E-Ausgaben (Aufwendungen für "volkswirtschaftlich wertvolle Erfindungen", F\&EAusgaben laut OECD-Definition) zur Anwendung.

Gegenüber dem Zustand vor der Reform wird kein Unternehmen schlechter gestellt, es gibt also keine Verlierer. Dem steht gegenüber, dass das Instrumentarium von Reform zu Reform komplexer wird. Ein Mehr an Komplexität erhöht in der Tendenz die Kosten der Administration und die "Compliance"-Kosten für Unternehmen.

\subsubsection{Empirische Evidenz zum Forschungsfreibetrag}

Die tatsächliche Nutzung und Wirkung des FFB ist wenig transparent: Eine Evaluierung wurde bisher nicht vorgelegt. Regelmäßig veröffentlicht werden - im Rahmen der jährlichen Förderungsberichte des Bundesministeriums für Finanzen - Schätzungen des Ausfalls von Steuereinnahmen aufgrund $\S 4$ Abs. 4 EStG (FFB, Spendenbegünstigung für Wissenschaft und Forschung). Im Förderungsbericht 2000 werden die dadurch bedingten Mindereinnahmen mit insgesamt 1,2 Mrd. S (87 Mio. €) im Jahr 2000 angegeben. Der Bundeshaushalt war nach dieser Schätzung im Jahr 2000 mit 800 Mio. S (58 Mio. $€$ ) betroffen. Dabei ist davon auszugehen, dass die Effekte der Erhöhung des FFB im Rahmen der Steuerreform 2000 nicht schon im ersten Jahr wirksam wurden. Die zusätzlichen Steuerausfälle aus der Reform 2002 beziffert das Bundesministerium für Finanzen (2002, S. 2) mit 73 Mio. $€$ pro Jahr (ab 2003).

Zur Abbildung der Generosität steuerlicher Anreize für F\&E für ein repräsentatives, der Körperschaftsteuer unterliegendes Unternehmen verwendet die OECD den eigens für diesen Zweck konstruierten "B-Index"13). Der B-Index reflektiert den Barwert des Gewinns vor Steuern, den das Unternehmen erzielen muss, um die Kosten einer Investition in F\&E zu decken und die entsprechenden Gewinnsteuern zu zahlen. Der B-Index ist gleich 1, wenn für die F\&EAusgaben keine steuerlichen Begünstigungen bestehen, die über deren sofortige Geltendmachung hinausgingen. Je "generöser" der steuerliche Anreiz für F\&E, desto niedriger ist der B-Index, bzw. je niedriger der B-Index, desto größer ist der Anreiz für ein Unternehmen in F\&E zu investieren.

Nach der Steuerreform 2000 beträgt der B-Index für den FFB - unter der Annahme eines Diskontsatzes von $5 \%$ - je nach Anteil der zusätzlichen an den gesamten F\&E-Ausgaben des Unternehmens zwischen 0,867 und $0,871^{14}$ ). Damit schnitt Österreich bei den steuerlichen Anreize für F\&E - wie ein Länder-Ranking der OECD in bezug auf die Generosität der indirekten F\&E-Förderung (ergänzt um WIFO-Berechnungen; Tabelle 6.3) zeigt - bereits vor der Reform 2002 im internationalen Vergleich relativ gut ab. Der gute Rang bezüglich der Anreize für F\&E dient der Sicherung und Stärkung des Forschungsstandorts.

13 Zur Konstruktion des Index siehe OECD (2000); nähere Details bei Warda (2002).

14 In dem Umstand, dass dieses Intervall sehr klein ist, kommt zum Ausdruck, dass die Differenzierung des Fördersatzes (25\% generell gegenüber 35\% für zusätzliche F\&E-Ausgaben) nur eine geringfügige Differenzierung der faktischen Anreizwirkung zur Folge hat. Dies resultiert aus der Verwendung eines die Anreizwirkung verwässernden gleitenden Dreijahresdurchschnitts zur Ermittlung der zusätzlichen F\&E-Ausgaben. 
Tabelle 5-3: Ranking nach der Generosiät der steuerlichen Anreize für F\&E (B-Index), 1999-2000

\begin{tabular}{|c|c|c|c|c|}
\hline & \multicolumn{2}{|c|}{ Große Unternehmen } & \multicolumn{2}{|c|}{ Kleine Unternehmen } \\
\hline & B-Index & Rang & B-Index & Rang \\
\hline Spanien & 0.687 & 1 & 0.687 & 4 \\
\hline Kanada & 0.827 & 2 & 0.678 & 3 \\
\hline Portugal & 0.850 & 3 & 0.850 & 6 \\
\hline Österreich & $0.867-0.871$ & 4 & $0.867-0.871$ & 7 \\
\hline Dänemark*) & 0.871 & 5 & 0.871 & 8 \\
\hline Australien & 0.890 & 6 & 0.890 & 10 \\
\hline Niederlande & 0.904 & 7 & 0.642 & 2 \\
\hline Frankreich & 0.915 & 8 & 0.915 & 11 \\
\hline Korea & 0.918 & 9 & 0.837 & 5 \\
\hline USA & 0.934 & 10 & 0.934 & 12 \\
\hline Irland & 0.937 & 11 & 0.937 & 13 \\
\hline Mexiko & 0.969 & 12 & 0.969 & 15 \\
\hline Japan & 0.981 & 13 & 0.937 & 14 \\
\hline Großbritannien & 1.000 & 14 & 0.888 & 9 \\
\hline Finnland & 1.009 & 15 & 1.009 & 17 \\
\hline Schweiz & 1.011 & 16 & 1.011 & 18 \\
\hline Belgien & 1.012 & 17 & 1.008 & 16 \\
\hline Griechenland & 1.015 & 18 & 1.015 & 19 \\
\hline Schweden & 1.015 & 19 & 1.015 & 20 \\
\hline Norwegen & 1.018 & 20 & 1.018 & 21 \\
\hline Italien & 1.027 & 21 & 0.552 & 1 \\
\hline Island & 1.028 & 22 & 1.028 & 22 \\
\hline Deutschland & 1.041 & 23 & 1.041 & 23 \\
\hline Neuseeland & 1.131 & 24 & 1.131 & 24 \\
\hline
\end{tabular}

Quelle: Warda (2002); Österreich: WIFO. *) Grundlagenforschung

Über die Wirkung des FFB liegen keine Untersuchungen vor. International wurde eine Vielzahl von Studien über Instrumente der steuerlichen F\&E-Förderung durchgeführt. Für das am besten untersuchte Instrument, den "R\&D Tax Credit" in den USA, kommt die Mehrzahl der neueren ökonometrischen Untersuchungen zu dem Ergebnis, dass ein Steuerausfall von $1 €$ zusätzliche F\&E-Ausgaben zwischen 1,3€ und 2,0€ induziert (siehe Hall - van Reenen, 2000).

Die steuerliche Förderung von F\&E in Österreich hat einen auch im international Vergleich beachtlichen Umfang erreicht und reicht an die direkte Technologieförderung heran. Dadurch entsteht ein gestiegenes Erfordernis, die Transparenz der steuerlichen Instrumente zu erhöhen. Internationale Beispiele für Evaluierungen von Instrumenten der steuerlichen Förderung von F\&E liegen vor. 


\section{Zur technologischen Leistungsfähigkeit des Unternehmenssektors}

Die technologischen Stärken - aber auch Schwächen - des österreichischen Unternehmenssektors sind wohlbekannt und wurden bereits in früheren Ausgaben des Forschungs- und Technologieberichts beschrieben: Verglichen mit dem Durchschnitt der EU-Länder fällt auf, dass die österreichische Wirtschaftsstruktur einen deutlich höheren Anteil arbeitsintensiver Produktion aufweist. Dem steht ein geringerer Anteil technologiegestützter Industrien gegenüber (vgl. Forschungs- und Technologiebericht 2001). Begriffe wie „Technologielücke“ oder „Struktur-Performance-Paradoxon“ (vgl. Hutschenreiter und Peneder 1997, Peneder 2001) verweisen auf diese strukturelle Schwäche der österreichischen Volkswirtschaft. Ähnliche Befunde lassen sich auch dem technologischen Profil des österreichischen Außenhandels ablesen (vgl. ARCS/WIFO 1998).

Der erste Teil untersucht anhand von Daten des US-Patentamtes, ob die Befunde zur sektoralen Spezialisierung der österreichischen Wirtschaft auch in Hinblick auf ihre Patentaktivitäten gültig ist. Die Strukturschwächen der österreichischen Wirtschaft haben neben ihren Auswirkungen für Wettbewerbsfähigkeit und Beschäftigung auch eine technologiepolitische Dimension. Es existiert ein starker Zusammenhang zwischen Wirtschaftsstruktur und der Höhe der gesamtwirtschaftlichen Ausgaben für Forschung und Entwicklung, der im zweiten Teil des Kapitels erörtert wird.

\subsection{Die Patentspezialisierung des Unternehmenssektors}

Wenn von der wissenschaftlichen und technologischen Leistungsfähigkeit des Unternehmenssektors gesprochen wird, so sind damit weniger hervorragende wissenschaftliche Leistungen und Publikationen gemeint. Im Gegensatz zur akademischen Forschung ist im Unternehmenssektor vielmehr entscheidend, wie gut wissenschaftliches und technologisches Wissen in neue Produkte und Dienstleistungen umgesetzt werden kann. Ein möglicher Indikator für diese Fähigkeit sind die Patentanmeldungen österreichischer Firmen, die im Folgenden untersucht und verglichen werden.

Anteilswerte bestimmter Branchen, wie sie oft für die Charakterisierung verschiedener nationaler Innovationssysteme gebräuchlich sind, liefern ein gutes Bild der Wirtschaftsstruktur. Sie sagen jedoch nur wenig über die tatsächlichen technologischen Kompetenzen der Unternehmen aus. Vielfach sind die verwendeten Branchengliederungen zu grob und neue Branchen wie etwa Biotechnologien nur unzureichend in den herkömmlichen Klassifikationen erfasst. Andererseits decken sich Branchenklassifikationen nur selten mit Technologiefeldern, da Unternehmen für gewöhnlich auf ein breites Feld verschiedener Technologien zurückgreifen und gewisse (generische) Technologien wie Lasertechnik, Informations- und Kommunikationstechnologien oder neue Werkstoffe in einer Reihe von Branchen zum Einsatz kommen. In der empirischen Technologieforschung werden daher oft Patentanmeldungen bzw. -erteilungen zur Charakterisierung des Umfangs und der Richtung (i.S. von technologischer Spezialisierung) der Genese neuen, technischen (kodifizierten) Wissens herangezogen. Obwohl Indikatoren auf 
Basis von Patenten mit einigen Nachteilen (vgl. z.B. Basberg 1986, Pavitt 1988) verbunden sind, lässt sich mit Hilfe derartiger Datenquellen ein international einigermaßen vergleichbares Bild der Wissensgenerierung zeichnen, dies insbesondere dann wenn auf Daten von sogenannten „repräsentativen“ Patentämtern zurückgegriffen wird. Dadurch können auch allfällige nationale Besonderheiten der patentrechtlichen Systeme ausgeglichen werden. Im Rahmen des vorliegenden Berichts wurden Daten des US-Patentamtes (USPO) verwendet, dessen globale Bedeutung zum Schutz geistigen Eigentums (IPR) unumstritten ist und die daher auch von der empirischen Technologieforschung zunehmend als Referenz herangezogen wird. Zu beachten ist, dass für das USPO (im Gegensatz zur Europäischen Patentamt in München) nur Daten hinsichtlich Patenterteilungen vorliegen. ${ }^{15}$

Patentanmeldungen am USPO werden nach ihrem zugehörigen Technologiefeld klassifiziert. Die Patente Österreichs wurden dabei zu etwa $10 \%$ in die Kategorie "sonstige Sachgüter" eingeordnet, gefolgt von Chemikalien, Maschinenbau und Textilien. Die Unterschiede zwischen diesem Patentprofil mit jenem der USA und der EU-Staaten zeigen, in welchen Technologiefeldern österreichische Unternehmen eine abweichende Anmeldungsdichte und damit höhere technologische Kompetenzen oder Defizite aufweisen.

Abbildung 6-1: Patentspezialisierung Österreichs: Unterschiede zum Spezialisierungsmuster der USA+EU in Prozentpunkten, 2000

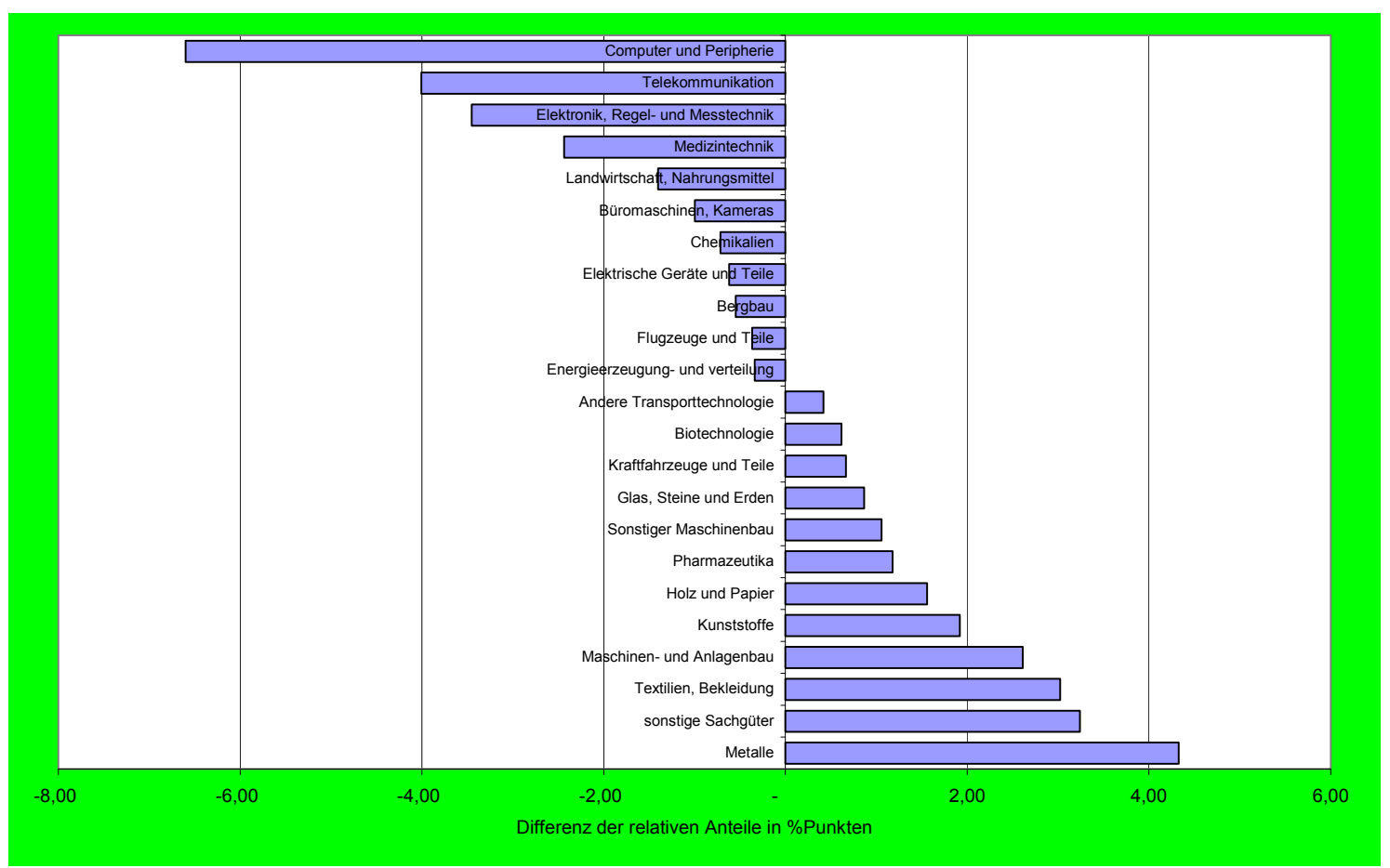

Quelle: CHI Research, eigene Berechnungen

15 Eine Darstellung der Position Österreichs anhand von Daten des Europäischen Patentamtes findet sich im Forschungs- und Technologiebericht 2001. 
Demnach sind österreichische Anmelder überproportional in Technologiefeldern wie Metallen, Textilien oder Maschinen- und Anlagenbau spezialisiert. Die größten Abstände zum Durchschnitt finden sich in der Zahl der Patentanmeldungen in den Bereichen Computer, Telekommunikation und Elektronik. Pharmazeutika und Biotechnologie sind die beiden einzigen Hochtechnologiebereiche, in denen die Patenthäufigkeit österreichischer Unternehmen etwa im Weltdurchschnitt liegt.

Wir finden hier also die Befunde zur technologischen Leistungsfähigkeit des österreichischen Unternehmenssektors, die auf Basis von Branchenanteilen (vgl. Peneder 2001) gemacht wurden, in groben Zügen bestätigt: Die österreichische Wirtschaft ist vor allem auf Produkte mit mittlerem Technologieniveau spezialisiert, während einige Hochtechnologiesektoren unterräpresentiert sind oder ganz fehlen.

Ein wesentlicher Vorteil der USPO-Daten liegt darin, dass hierfür auch bibliometrische Indikatoren (v.a. auf Basis von Zitationsanalysen, vgl. Narin, Olivastro 1988) vorliegen. Damit lässt sich die Position Österreichs nicht nur ausschließlich auf der Basis angemeldeter Patente, sondern auch in Bezug auf die wissenschaftlich-technologische Relevanz der Patentaktivitäten einschätzen. Dafür werden drei Indikatoren herangezogen:

- der „Current Impact Index“ gibt die normalisierte Zahl der Zitationen, die ein Patent erhält, an ( 1 = weltweiter Durchschnittswert). Patentschriften, die (überdurchschnittlich) oft zitiert werden, weisen also einen höheren „Impact“ auf, und es kann indirekt auf eine überdurchschnittliche Qualität bzw. technologische Bedeutung des Patents geschlossen werden.

- die „Technological Cycle Time“ gibt den Median des Alters der durch ein Patent zitierten anderen Patente an (d.h. je niedriger der Wert, desto jüngeren Datums sind die zitierten Patente). Bezieht sich ein Patent hauptsächlich auf Patente jüngeren Datums, wird gefolgert, dass das Patent auf neueste technologische Entwicklungen Bezug nimmt und somit auf dem neuesten Stand der Technologie entspricht.

- Die „Science Linkage“ bezeichnet die durchschnittliche Zahl an Zitationen wissenschaftlicher Literatur in einer Patentschrift. Patente mit einer intensiven Zitationsbeziehung zur wissenschaftlichen Literatur wird eine besondere Nähe zur wissenschaftlichen Forschung zugesprochen, was als Indikator für die gegenseitige Befruchtung bzw. Interdependenz von Wissenschaft und Technologie im betreffenden Feld angesehen werden kann.

In den 90er Jahren kam es zu einem deutlichen Anstieg der Patenterteilungen am USPO, wobei das Wachstum (in unterschiedlichem Ausmaß) von allen Ländern getragen wurde. ${ }^{16}$ Die Zahl der Patenterteilungen hat in der zweiten Hälfte der 90er Jahre gegenüber der ersten Hälfte (für

16 Aufgrund von OECD-Statistiken lässt sich seit geraumer Zeit ein Trend zur „Internationalisierung“ von intellectual property rights feststellen (die Zunahme der Patentanmeldungen/-erteilungen an nicht-nationalen Patentämtern nahm stärker zu als jene von Inländern an den jeweiligen nationalen Patentämtern). Im Fall des USPO hat sich aber gezeigt, dass auch die Patenterteilungen an amerikanischen Unternehmen/Erfindern im Zug der 90er Jahre deutlich gestiegen ist. Dies stellt insofern einen Trendbruch dar, als noch in den 80er Jahren eher eine Stagnation festzustellen war. 
die hier betrachteten Länder USA, EU-14 $4^{17}$, Schweiz) um mehr als ein Drittel zugenommen. Auch Österreich war an diesem Trend beteiligt: Mit ca. $35 \%$ Zunahme der Patenterteilungen am USPO erreichte Österreich annähernd die Wachstumsrate (36 \%) aller hier betrachteten Länder. Der Anteil österreichischer Patente an den gesamten Patentaktivitäten der EU-Länder am USPO betrug im Jahr 2000 2,3 \%, wobei dieser Anteil (von geringfügigen Schwankungen im Bereich von Zehntelprozentpunkten) im Zeitablauf konstant bleibt. Er liegt damit in etwa im Bereich des finnischen Anteils $(2,4 \%){ }^{18}$

Gleichzeitig lassen sich hinsichtlich der Entwicklung der genannten Patentindikatoren folgende Trends beobachten:

- Sowohl für Österreich als auch für die EU-14 lässt sich eine leicht sinkende Tendenz des „Current Impact Index" beobachten, während für die Patenterteilungen der USA die gegenläufige Entwicklung gilt. Das bedeutet, dass europäische und auch österreichische Patente tendenziell an Bedeutung als Quelle für technologisches Wissen in anderen Patenten verlieren. Auffällig ist hier das deutliche niedrigere Niveau des österreichischen Impact-Index gegenüber jenen der EU-14. Der große Abstand der EU gegenüber den USA wird vor allem durch die Stellung des USPO als nationales Patentamt der USA bedingt. ${ }^{19}$

- Der Indikator für den „technological life cycle“ weist in allen drei Vergleichsräumen eine leicht - sinkende Tendenz auf, d.h. die von Patenten zitierten Patentschriften werden im Durchschnitt immer jüngeren Datums. Österreichische Patente zitieren im Vergleich zu den USA bzw. zu den EU-14 tendenziell Patente, die im Durchschnitt ein bis zwei Jahre älter sind. Dies ist ein Hinweis darauf, dass Österreich auf vergleichsweise „ältere“ technologische Entwicklungen Bezug nimmt.

- Der „science linkage“ der Patente am USPO nahm in den 90er Jahren allgemein stark zu. Die Interdependenz zwischen Wissenschaft und Technologie hat sich somit im betrachteten Zeitraum deutlich erhöht. Österreich (wie auch die EU-14 Länder im Durchschnitt) liegen auch bezüglich dieses Indikators unter dem Niveau der USA.

17 EU-14: ungewichtetes arithmetisches Mittel der EU-Länder mit Ausnahme Luxemburgs.

18 Allerdings soll darauf hingewiesen werden, dass Finnland im Zuge der 90er Jahre eine außerordentlich dynamische Entwicklung hinsichtlich der Zahl der Patenterteilungen am USPO erzielen konnte. Der Anteil finnischer Patente an der Gesamtzahl der EU-14-Patente stieg dadurch von 1,8 \% im Jahr 1990 auf die erwähnten 2,4 \% im Jahr 2000.

19 Es konnte festgestellt werden, dass Patente von Anmeldern, die aus dem gleichen Land kommen, tendenziell häufiger zitiert werden. Teilweise konnte ein Einfluss der geographischen Nähe identifiziert werden (z.B. Jaffe et al. 1993). Da am USPO naturgemäß US-amerikanische Patentanmelder dominieren, ergibt sich gleichsam zwangsläufig ein höheres Niveau für US-Patente, was den Zitationsimpact betrifft. 
Abbildung 6-2: Entwicklung von Patentindikatoren am USPO
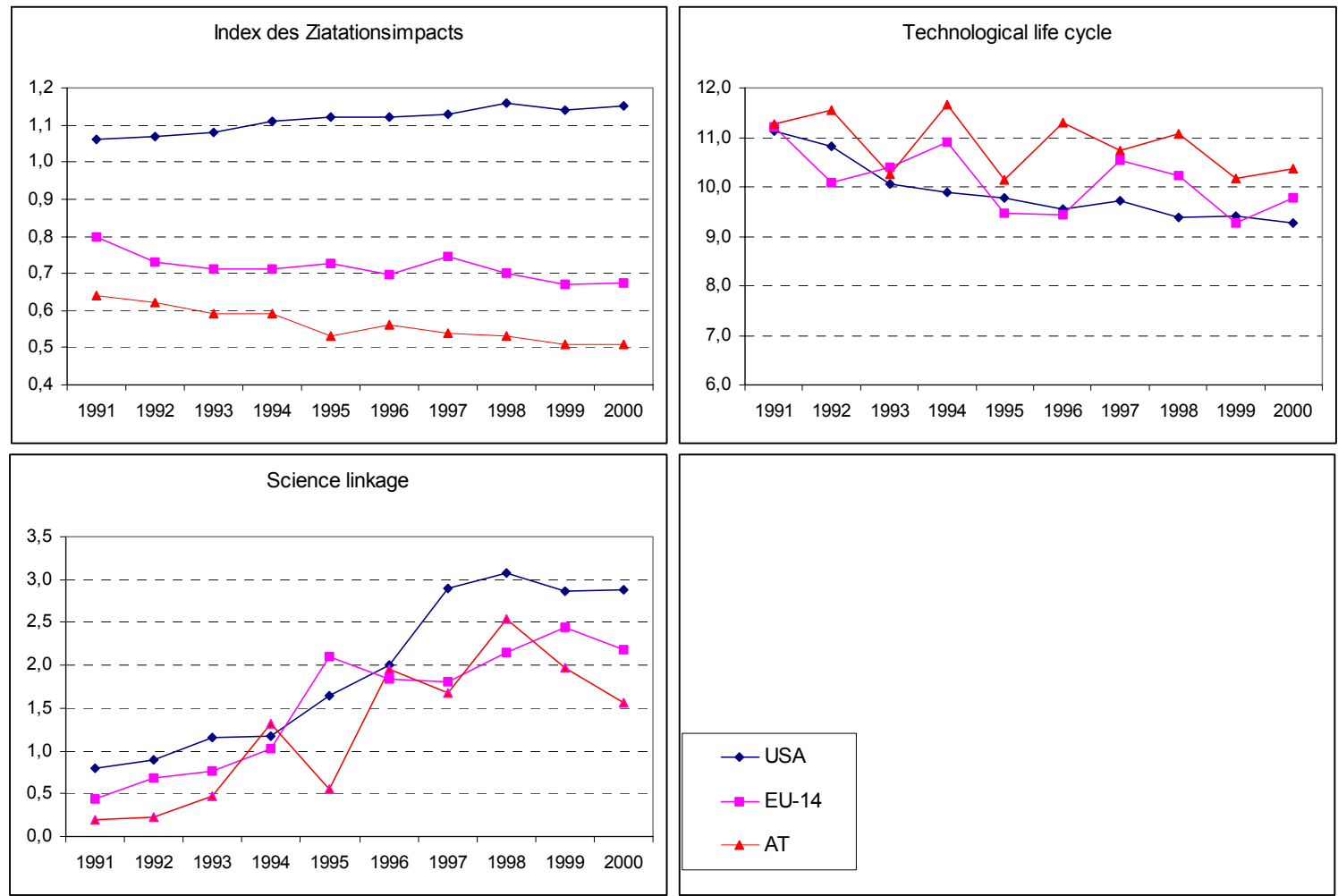

Quelle: CHI-Research, eigene Berechnungen

Das eher ungünstige Bild für Österreich zeigt sich auch in einem detaillierteren Ländervergleich:

- Der „Impact“ österreichischer Patenterteilungen ist sehr gering. Tatsächlich liegt Österreich, was diesen Indikator betrifft, an letzter Stelle aller hier erfassten Länder. Ein naheliegender Schluss ist hier, dass die österreichischen Patentierungen technologisch nicht für andere Anwender interessant sind. Das kann entweder die Folge einer übermäßigen Spezialisierung, aber auch die Folge eines geringeren technologischen Niveaus sein.

- Österreichische Patente zitieren geringfügig ältere Patente (hoher „technological life cycle“). Nur Dänemark und Portugal schneiden in Bezug auf diesen Indikator noch schlechter ab. Dies könnte ein Hinweis auf die Spezialisierung der österreichischen Patentinhaber auf eher traditionelle Sektoren sein, in denen die Geschwindigkeit des technologischen Wandels geringer ist.

- Die Verbindung zur Grundlagenforschung (bzw. zum Wissenschaftssystem) ist in Österreich nur mittelmäßig ausgeprägt. Auch hier liegt der Schluss nahe, dass darin ein Hinweis auf eine Spezialisierung in Sektoren zu sehen ist, in denen vergleichsweise wenig Impulse aus der aktuellen wissenschaftlichen Forschung kommen. 
Abbildung 6-3: Qualitätsindikatoren von Patenterteilungen am USPO im internationalen Vergleich
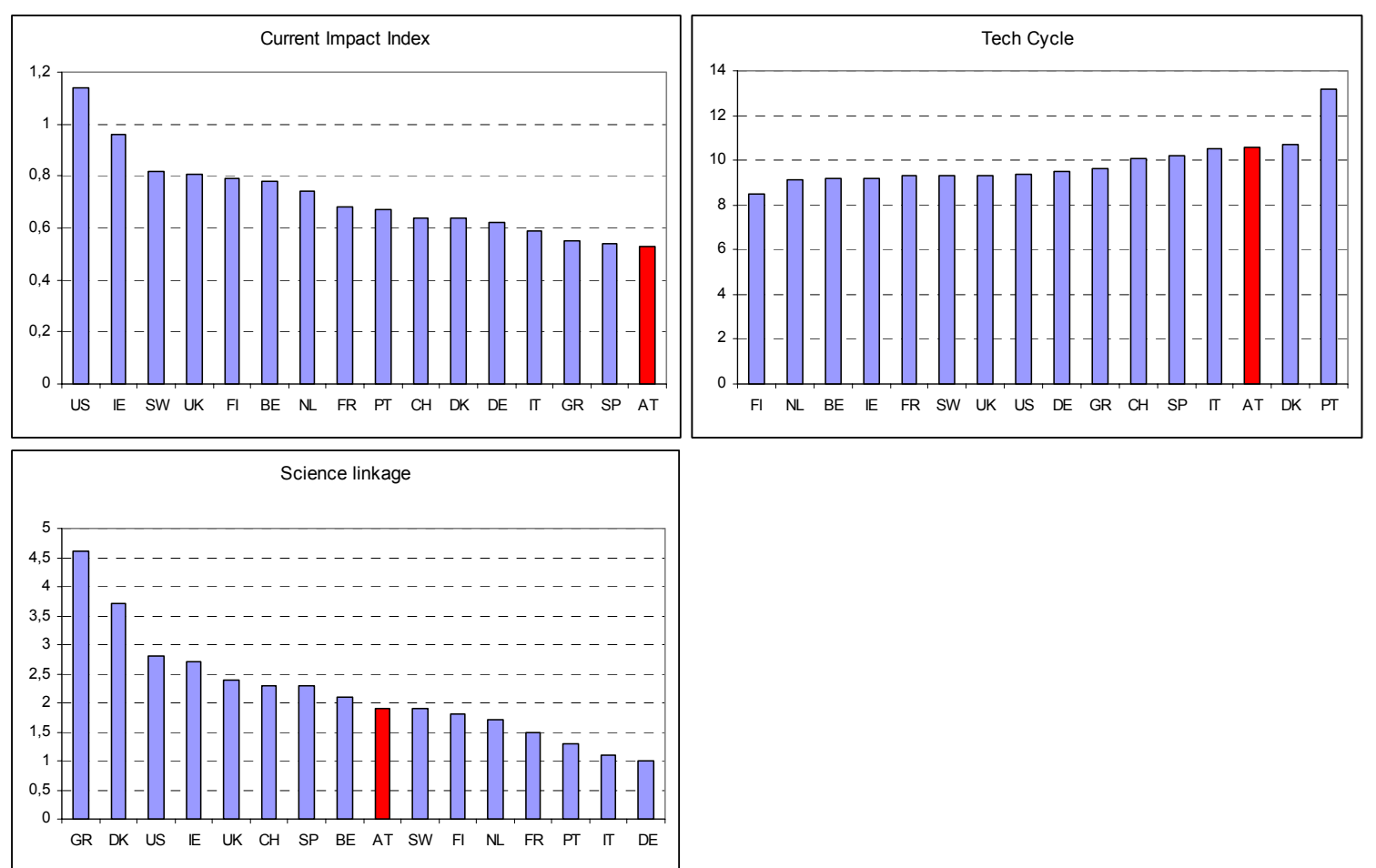

Quelle: CHI Research, eigene Berechnungen

Österreichische Patente werden nur selten zitiert, zitieren selbst vergleichsweise ältere Patente und beziehen sich deutlich weniger als die Patente anderer Länder auf wissenschaftliche Literatur.

In Abbildung 6-4 ist ein Portfolio der österreichischen Patentlandschaft am USPO dargestellt, das sowohl statische wie auch dynamische Elemente berücksichtigt. Das statische Element bezieht sich auf die relative Spezialisierung Österreichs in einem bestimmten Technologiefeld. Es wird auf der X-Achse durch einen Spezialisierungsindikator, den sogenannten RCA-Wert abgebildet. Formal ist der RCA-Wert folgendermaßen definiert:

$$
R C A_{i}=\frac{\frac{P_{i j}}{\sum_{j} P_{i j}}}{\sum_{i} P i j}
$$


wobei:

P Zahl der Patenterteilungen am USPO

i $\quad$ Land $(i=1, \ldots, 16)$

j Technologiefeld $(j=1, \ldots, 32)$

Ein RCA-Wert $>1$ bedeutet, dass ein Land in dem betreffenden Technologiefeld überproportional spezialisiert ist und vice versa.

Die dynamische Komponente wird mit der Wachstumsdifferenz der Entwicklung der Patenterteilungen Österreichs zu jener aller hier betrachteten Länder (USA plus EU-Länder) abgebildet:

$$
\Delta W_{j, A U T}=W_{j, A U T}-W_{j, \text { alleLänder }}
$$

wobei $\mathrm{W}_{\mathrm{j}}$ das prozentuelle Wachstum der Patenterteilungen zwischen den beiden Perioden 1991 - 1995 und 1996 - 2000 in einem Technologiefeld j bezeichnet. Positive Werte zeigen also ein überdurchschnittliches Wachstum der österreichischen Patenterteilungen am USPO in den betreffenden Technologiefeldern an und vice versa.

Zusätzlich zeigt die Größe der Kreise in Abbildung 6-4 auch noch die absolute Zahl an Patenterteilungen Österreichs am USPO in den Jahren 1996 bis 2000 an.

\section{Abbildung 6-4: Patentspezialisierungsportfolio Österreichs am USPO}

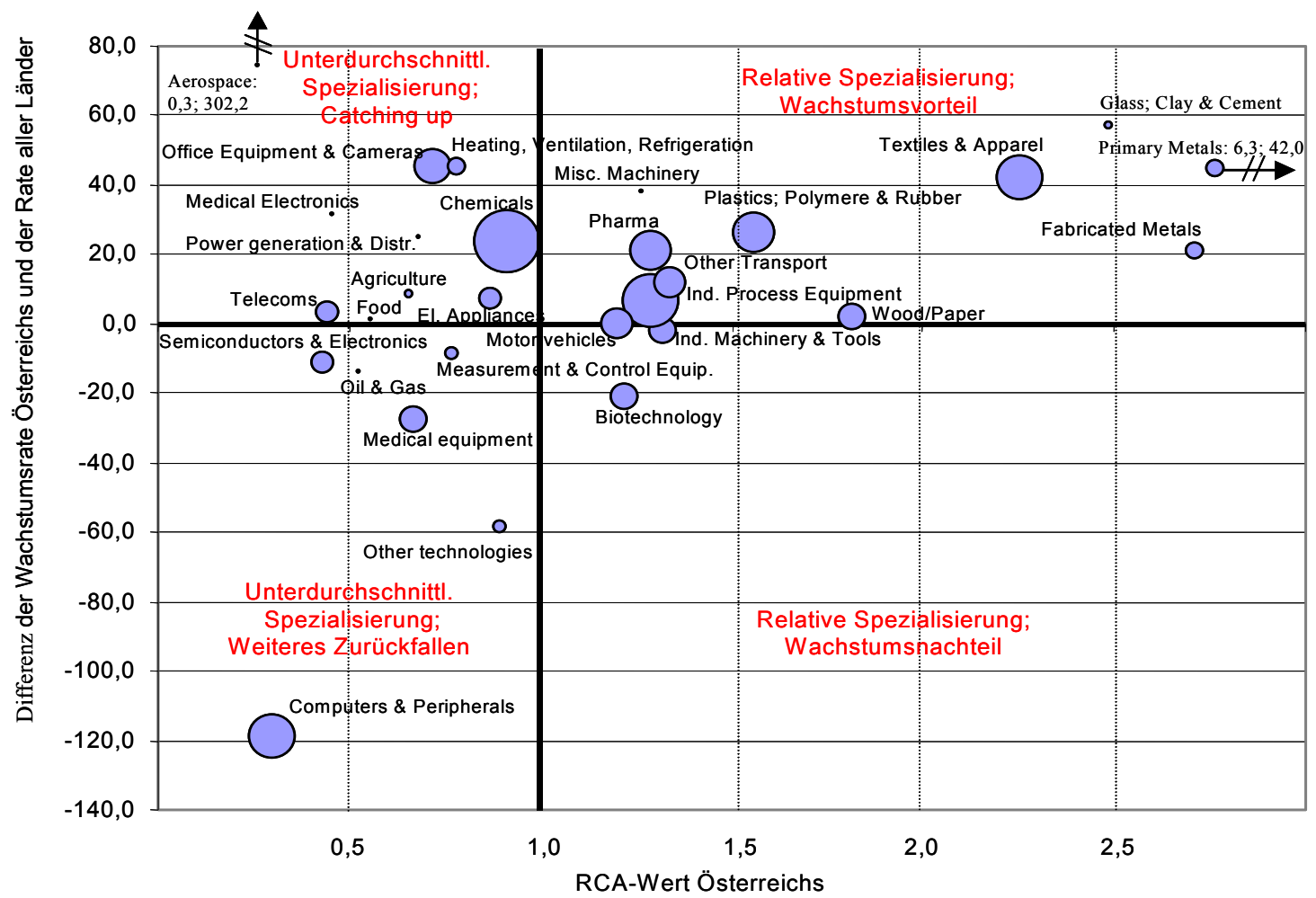

Quelle: CHI Research, eigene Berechnungen 
Daraus ergibt sich eine Struktur mit vier Quadranten: Bei Technologiefeldern, die im nordöstlichen Quadranten liegen, weist Österreich sowohl eine überdurchschnittlich hohe Spezialisierung (RCA-Wert > 1) und ein stärkeres Wachstum auf. D.h., dass es in diesen Technologiefeldern zu einer im Zeitablauf zunehmenden Spezialisierung Österreichs kommt (und zwar ausgehend von einem bereits existierenden Spezialisierungsvorteil). Umgekehrt gilt für den südöstlichen Quadranten, dass Österreich in diesen Technologiefeldern zwar einen Spezialisierungsvorteil aufweist, das Wachstum der Patenterteilungen Österreichs ist allerdings geringer als im Durchschnitt aller hier betrachteten Länder. Für den nordwestlichen Quadranten gilt, dass in diesen Technologiefeldern Österreich eine unterdurchschnittliche Spezialisierung aufweist, es allerdings zu einem Aufholprozess kommt, da das Wachstum der Patenterteilungen überdurchschnittlich ist. Hingegen ist Österreich in den Technologiefeldern des südwestlichen Quadranten sowohl unterdurchschnittlich spezialisiert und verliert auch aufgrund einer unterdurchschnittlichen Wachstumsrate weiter an Boden.

Die wesentlichsten Schlussfolgerungen aus dieser Portfolio-Betrachtung lassen sich wie folgt zusammenfassen:

- Österreich ist bei bestimmten, eher traditionellen Technologiefeldern wie Textilien, Kunststoffen, Metallen, Holz und Papier hochspezialisiert. Zu diesen Feldern gehören auch Pharmazeutika und industrielle Prozesstechnologie.

- In Technologiefeldern, in denen Österreich einen relativen Spezialisierungsvorteil aufweist, ist tendenziell auch die Wachstumsrate Österreichs überdurchschnittlich. Dies lässt auf eine zunehmende Spezialisierung auf bestimmte technologische Kernkompetenzen schließen. Dort, wo Österreich bereits Stärken aufweisen kann (im Sinne einer überdurchschnittlichen relativen Spezialisierung), werden diese Stärken weiter ausgebaut.

- Die (wenigen) Technologiefelder, die im südöstlichen Quadranten liegen (Biotechnology; Motor/Vehicles; Industrial Machinery \& Tools) weisen lediglich einen geringen Wachstumsrückstand auf. ${ }^{20}$

- Gleichzeitig finden sich eine Reihe von - quantitativ - bedeutsamen Technologiefeldern (z.B. Chemicals; Office Equipment), in denen Österreich zwar eine unterdurchschnittliche Spezialisierung aufweist, deren Wachstumsrate jedoch deutlich über dem Durchschnitt der Vergleichsländer liegt. Diese können somit als "catching-up Technologiefelder" eingestuft werden.

- Technologiefelder, in denen Österreich sowohl einen Wachstumsrückstand als auch eine unterdurchschnittliche Spezialisierung aufweist, sind zu einem Gutteil dem IKT-Sektor i.w.S. zuzurechnen: Computers \& Peripheral; Semiconductors \& Electronics sowie Medical Euqipment. ${ }^{21}$

20 Hinsichtlich der Einschätzung der negativen Wachstumsdifferenz Österreichs im Technologiefeld Biotechnologie von 20 Prozentpunkten ist zu berücksichtigen, dass dieses Technologiefeld mit etwas über $180 \%$ (bezogen auf alle hier berücksichtigen Länder) ein überaus starkes Wachstum aufweist. Die Patenterteilungen Österreichs nehmen hier daher ebenfalls sehr stark zu, jedoch in etwas geringeren Ausmaß als in den Vergleichsländern.

21 Die geringe Performance sowohl in Struktur als auch in Dynamik des Technologiefeldes Computers \& Peripherals ist Ausdruck des nur sehr kleinen Sektors hardwareerzeugender Unternehmen in Österreich (vgl. dazu auch den Forschungs- und Technologiebericht 2001, sowie Gassler, Rammer und Polt, 1999). 
Alles in allem ergibt sich ein Bild, das die These von der Pfadabhängigkeit technoökonomischer Spezialisierung bestätigt: Technologiefelder mit RCA-Werten Österreichs von $>1$ wachsen tendenziell im internationalen Vergleich überdurchschnittlich. Wachstumsrückstände weist Österreich fast ausschließlich in Technologiefeldern auf, die in Österreich auch relativ "schwach“ vertreten sind. Erfreulich ist die Tatsache, dass in einer Reihe von Technologiefeldern trotz RCA-Werten von < 1 ein überdurchschnittliches Wachstum zu verzeichnen ist. ${ }^{22}$ Positiv zu bewerten ist weiters die Tatsache, dass im südöstlichen Quadranten kaum Technologiefelder zu finden sind, was bedeutet, dass Österreich seine technologischen Stärken im Zeitablauf ausbauen konnte.

\subsection{Forschung, Technologie und Wirtschaftsstruktur}

Wie in Kapitel 3 gezeigt, weisen Länder mit hoher Forschungsquote auch überdurchschnittliche Finanzierungsanteile der Unternehmen am GERD auf. Der Grund dafür ist, dass Zuwächse bei den gesamtwirtschaftlichen F\&E-Aufwendungen im vergangenen Jahrzehnt fast ausschließlich durch Investitionen des Unternehmenssektors getragen wurden, während die Aufwendungen des staatlichen Sektors in vielen Ländern stagnierten oder zurückgingen.

Das Wachstum der F\&E-Ausgaben im Unternehmenssektor wird jedoch nicht von allen Branchen gleichmäßig getragen. Technologischer Wandel auf Unternehmensebene wird neben firmenspezifischen Faktoren auch stark durch branchenabhängige Einflüsse bestimmt. So unterscheiden sich Branchen stark in ihrem Marktwachstum, den technologischen Möglichkeiten und Aneignungsbedingungen sowie der kumulativen Natur der zugrundeliegenden technologischen Wissensbasis. Die Kombination dieser Faktoren bestimmen wesentlich die Anreize zu Forschung und Innovation und damit auch die F\&E-Intensität auf Branchenebene. Verschiedene Autoren (vgl. Pavitt 1984, Peneder 1999, Marsili 2001, OECD 2001) haben deshalb Branchentaxonomien erarbeitet, die diese unterschiedliche Forschungs- und Technologieintensiät abzubilden versuchen. So unterscheidet etwa die OECD zwischen vier Kategorien ${ }^{23}$ :

$22 \mathrm{Zu}$ beachten ist hier wiederum, dass das Wachstum (zwischen den Perioden 1989-94 und 1995-2000) der österreichischen Patenterteilungen am USPO mit ca. $35 \%$ beinahe identisch mit jenem der Summe der hier betrachteten Länder ist (ca. $36 \%$ ).

23 High-technology: Herstellung von Luft- und Raumfahrtzeugen, Herstellung von Pharmazeutika, Herstellung von Büromaschinen und Datenverarbeitungsgeräte, Herstellung von Rundfunk- Fernseh- und Nachrichtengeräten.

Medium-high-technology: Herstellung von Geräten zur Elektrizitätserzeugung und -verteilung, Herstellung von Kraftwagen, Herstellung von Schienenfahrzeugen, chemische Industrie ohne Pharmazeutika, Maschinenbau.

Medium-low-technology: Mineralölverarbeitung, Herstellung von Gummi- und Kunststoffwaren, Herstellung von Produkten aus Steinen und Erden, Schiffsbau, Metallerzeugung, Herstellung von Metallerzeugnissen.

Low -technology: Herstellung von Textilien, Lederwaren und Schuhen, Herstellung von Nahrungsmitteln und Getränken, Tabakverarbeitung, Be- und Verarbeitung von Holz, Herstellung und Bearbeitung von Papier und Pappe, Verlagswesen, Druckerei, Vervielfältigung, Herstellung von sonstigen Erzeugnissen

vgl. OECD 2001, Annex 
- High-technology-industries (HT)

- Medium-high-technology industries (MHT)

- Medium-low-technology-industries (MLT)

- Low-technology-industries (LT)

Ein Vergleich der Anteile dieser Branchengruppen an der gesamtwirtschaftlichen Wertschöpfung oder Beschäftigung lässt Rückschlüsse auf das technologische Profil des Landes zu. Die Position Österreichs in diesem Vergleich bestätigt einmal mehr die bekannten Strukturbefunde. Der Wertschöpfungsanteil von HT- und MHT-Industrien an der gesamten österreichischen Sachgüterproduktion liegt sowohl unter dem Durchschnitt der OECD als auch unter dem der EU-Staaten. Das Profil Österreichs ist am ehesten mit Italien, Frankreich oder Kanada vergleichbar.

Besonders auffällig ist, dass einige Länder mit bedeutend niedrigerer Wirtschaftleistung inzwischen einen wesentlich höheren Anteil an Hochtechnologiebranchen als Österreich aufweisen. Zu dieser Gruppe gehören vor allem Irland, aber auch einiger mittel- und osteuropäischer Nachbarländer Österreichs wie Ungarn oder die Tschechischen Republik. Vor allem am Beispiel Irlands zeigen sich hier die Vorteile von ,fast seconds', die durch eine gezielte Förderungs- und Ansiedelungspolitik einen raschen Strukturwandel durchmachen.

Abbildung 6-5: Anteil von Hochtechnologiebranchen in den OECD-Ländern, 1998

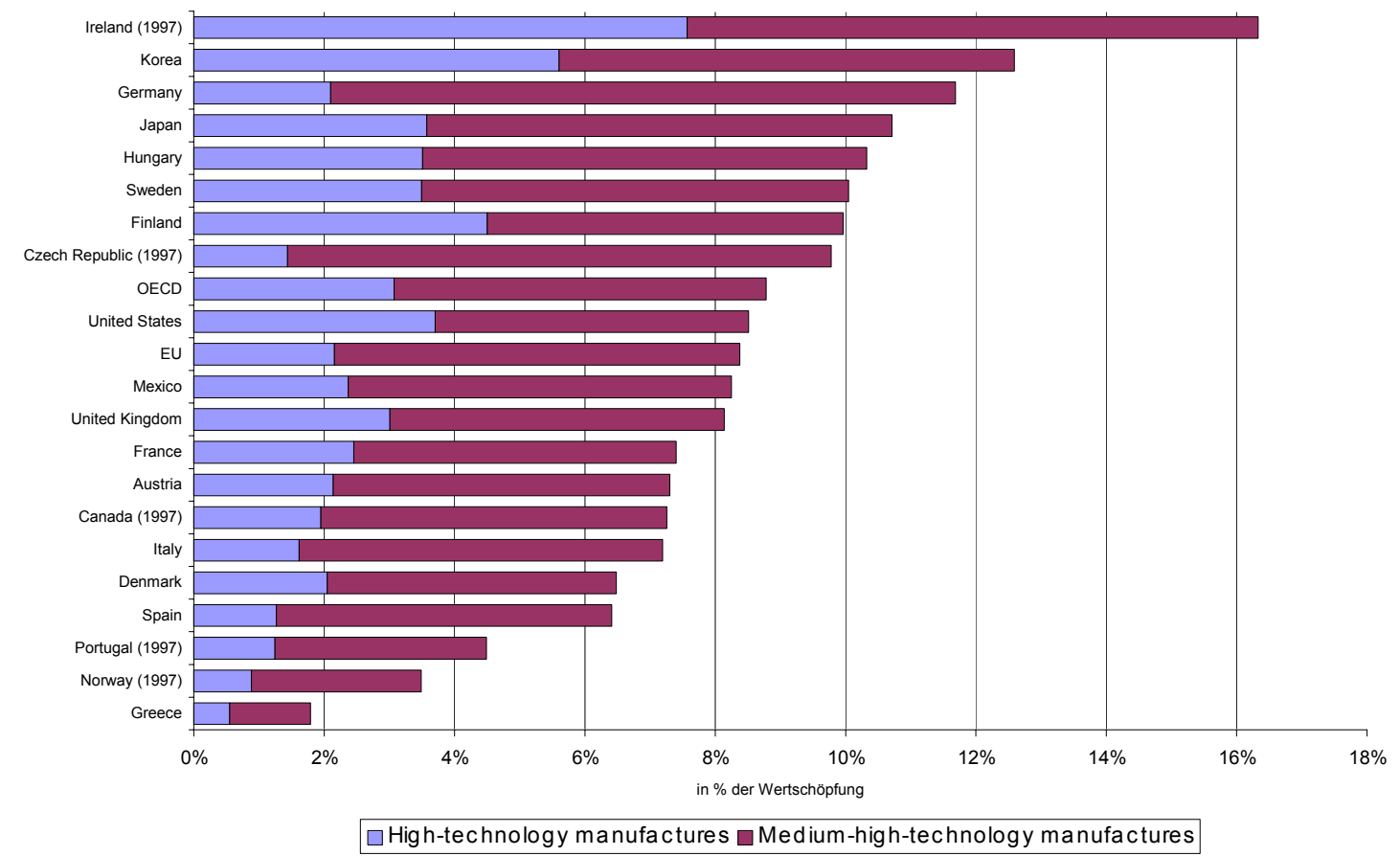

Quelle: OECD 2001, Statistik Austria 
Die Strukturunterschiede Österreichs im Vergleich mit dem EU-Durchschnitt bestehen vor allem in niedrigeren Anteilen der chemischen Industrie, des Kfz-Baus und der Herstellung von Elektrizitätsverteilungs- und -schalteinrichtungen. Wesentlich unterrepräsentiert ist außerdem der Luft- und Raumfahrzeugbau. Dagegen findet sich eine höhere Konzentration vor allem in grundstoffnahen Bereichen wie der Erzeugung und Verarbeitung von Metallen, Holz, Papier oder Pappe.

Übertragen auf die OECD- oder WIFO-Klassifikation (vgl. Peneder 1998) hat Österreich also Strukturnachteile in Hochtechnologiebranchen bzw. technologiegestützten Sektoren und Strukturvorteile in Mittel- und Niedrigtechnologiebranchen bzw. arbeits- und kapitalintensiven Sektoren. A priori bedeuten diese Strukturunterschiede aus wirtschaftspolitischer Perspektive noch keinen Vor- oder Nachteil, obwohl empirische Studien auf einen Zusammenhang zwischen Strukturvorteilen und Wachstum hinweisen (vgl. Peneder et al. 2001). Kompetitive Vorteile können auch auf einer gelungenen Spezialisierung in Nischen, einer günstigen Ressourcenausstattung oder anderen Standortvorteilen beruhen.

Aus forschungs- und technologiepolitischen Perspektive sind diese Strukturnachteile jedoch schwerwiegend, denn in den OECD-Ländern konzentrieren sich die F\&E-Aufwendungen zum größten Teil (88\%) auf HT- und MHT-Sektoren (vgl. OECD 2001). Auch in Österreich wird über 80\% der F\&E in diesen Bereich der Wirtschaft geleistet. Fehlen diese Branchen, so ist zu vermuten, dass auch die gesamtwirtschaftliche F\&E-Quote nicht an jene anderer Länder herankommen wird.

Die österreichische Wirtschaft hat Strukturnachteile in Hochtechnologiebranchen bzw. "technology-driven industries". Strukturvorteile hat Österreich vor allem in grundstoffnahen Bereichen wie der Erzeugung und Weiterverarbeitung von Metallen, Holz, Papier oder Pappe. Aus forschungs- und technologiepolitischer Perspektive sind diese Nachteile schwerwiegend, da in diesen Branchen ein Großteil der F\&E-Aufwendungen konzentriert sind.

Diese Vermutung soll anhand eines Strukturportfolios, das F\&E-Intensität und die relativen Anteile verschiedener Branchen der österreichischen Sachgüterproduktion mit den EUDurchschnittswerten vergleicht, überprüft werden. Auf der horizontalen Achse ist die Abweichung des Anteilswerts des jeweiligen Sektors an der Gesamtwirtschaft zwischen Österreich und EU, auf der vertikalen Achse die Abweichung der F\&E-Intensität des Sektors vom EU-Durchschnitt angegeben. Horizontale und vertikale Achse teilen die Zeichenfläche in vier Quadranten. Die Technologieniveaus nach OECD (2001) sind durch die Farben orange (HT, MHT), grün (MLT) und blau (LT) gekennzeichnet. Zusätzlich zeigt der Radius des Kreises die absolute Höhe der F\&E-Aufwendungen der jeweiligen Branche in der Europäischen Union.

Würde die österreichische Industrie in ihrer Zusammensetzung und ihren jeweiligen F\&EIntensitäten genau dem europäischen Durchschnitt entsprechen, so wären alle Branchen in der Mitte des Diagramms positioniert. Tatsächlich findet sich jedoch kaum eine in der Mitte wieder. Die österreichische Industrie hat in eine Reihe von Spezialisierungsvorteilen, was an der Position verschiedener Sektoren in den beiden oberen Quadranten abzulesen ist. Diese Vorteile finden sich jedoch meist in Sektoren mit mittleren (grün) und niedrigen (blau) 
Technologieniveaus. Es handelt sich dabei um die Erzeuger traditioneller Industriegüter wie Papier, Pappe, die holz- oder metallverarbeitende Industrie. Die betreffenden Branchen haben im Vergleich zum EU-Durchschnitt oftmals auch eine leicht überdurchschnittliche F\&EIntensität. Österreichische Firmen in diesen Bereichen können auf über lange Jahre aufgebaute technologische Kompetenz setzen und behaupteten sich auf ihren jeweiligen Märkten bisher gut (vgl. Peneder 2001).

\section{Abbildung 6-6: Strukturportfolio für die österreichische Sachgüterproduktion, 1998}

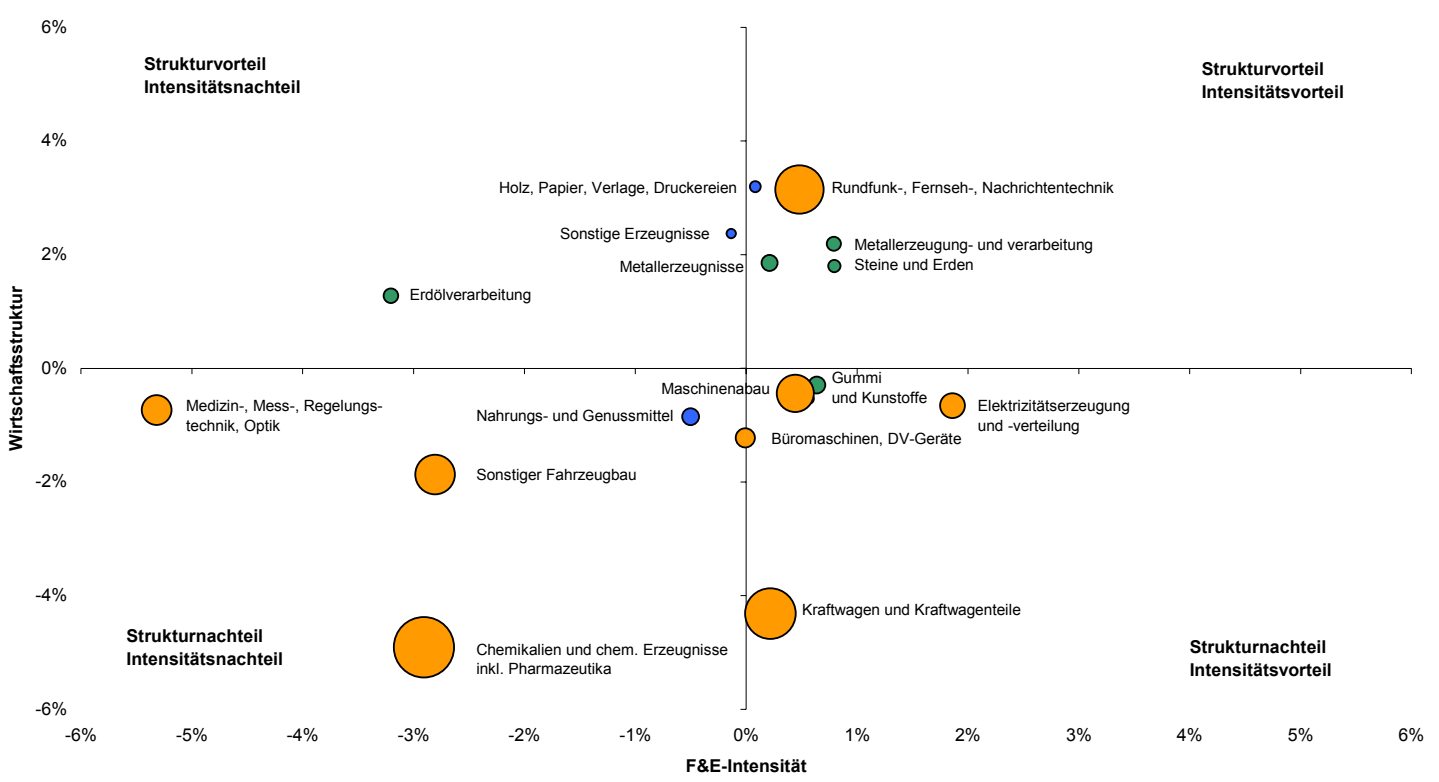

Quelle: EUROSTAT New Cronos Datenbank, OECD 2001, (EU-Werte 1997), Statistik Austria (2002), eigene Berechnungen

Im Gegensatz dazu weist Österreich in HT- oder MHT-Branchen (orange) dagegen häufig Strukturnachteile auf, wie aus ihrer Position in den beiden unteren Quadranten zu erkennen ist. $\mathrm{Zu}$ diesen unterrepräsentierten Branchen gehörten vor allem die chemische Industrie inkl. der Pharmaindustrie (NACE 24), Medizin-, Mess-, Regelungstechnik, Optik (NACE 34) und der sonstige Fahrzeugbau (NACE 35), der auch die besonders F\&E-intensive Luft- und Raumfahrtindustrie enthält. Die Dichotomie des Diagramms (HT- und MHT-Branchen in der oberen, MLT- und LT-Branchen in der unteren Hälfte) führt noch einmal die „Technologielücke“ Österreichs plastisch vor Augen. Nur ein Hochtechnologiesektor, Rundfunk-, Fernseh- und Nachrichtentechnik (NACE 32), liegt im oberen Teil der Grafik.

Die technologiepolitischen Schlussfolgerungen dieses Diagramms illustriert ein kleines Gedankenexperiment. Würde das Problem der niedrigen Technologieorientierung der heimischen Wirtschaft allein darauf beruhen, dass die einzelnen Unternehmen weniger als ihre EU-Konkurrenten forschen, wären alle Branchen knapp über oder unter der horizontalen Achse angeordnet. In diesem Fall könnte ein technologischer Nachteil der österreichischen Wirtschaft durch verstärkte F\&E-Förderung wettgemacht werden. Tatsächlich aber weichen einige 
Sektoren, vor allem die Hochtechnologiesektoren Chemie, Medizin- Mess- und Regeltechnik und sonstiger Fahrzeugbau weit von der Mittelachse nach unten ab. Zusätzlich verstärken sich die Strukturdefizite durch eine niedrigere F\&E-Intensität, sodass eine Reihe von wichtigen Hochtechnologiebranchen im oder nahe dem linken unteren Quadranten, der sowohl auf einen Struktur- als auch F\&E-Rückstand hinweist, zu finden sind. Der technologiepolitische Handlungsbedarf ist also durch ein Intensitäts- und ein Strukturproblem begründet.

In dieser Perspektive würde die niedrigere F\&E-Quote Österreichs weniger auf die mangelnde Innovationsneigung der heimischen Wirtschaft oder fehlende Anreize und Förderungen, sondern auf die Wirtschaftsstruktur zurückzuführen sein. Dieser Zusammenhang zwischen der nationalen F\&E-Quote und der Wirtschaftsstruktur wird zusätzlich durch den Umstand verstärkt, dass diese unterrepräsentierten Sektoren innerhalb der EU zu jenen mit den höchsten absoluten F\&E-Aufwendungen gehören, was an der Größe der Kreise zu erkennen ist. Darin zeigt sich der starke Zusammenhang von Forschungs- und Technologiepolitik zu allgemeineren wirtschaftspolitischen Fragen wie Strukturwandel und Gründungsdynamik. Dieser Zusammenhang ist im Übrigen nicht neu. Schon Schumpeter hat auf den engen Zusammenhang zwischen Innovation, wirtschaftliche Entwicklung und Strukturwandel hingewiesen.

Die im internationalen Vergleich niedrige F\&E-Quote Österreichs kann zu einem wesentlichen Teil durch die Struktur der heimischen Wirtschaft erklärt werden. Zwar liegen in einer Reihe von Branchen die F\&E-Intensitäten auf oder über dem europäischen Niveau. Es fehlen jedoch einige besonders forschungsintensive Hochtechnologiebereiche.

Eine aktive Struktur- und Gründungspolitik könnte in Abstimmung mit F\&E- und Innovationsförderung Anteilsveränderungen hin zu stärker technologieintensiven Branchen durch eine höhere Wachstumsdynamik in diesen Sektoren unterstützen. Ein solcher intrasektoraler Strukturwandel weg von traditionellen, grundstoffbasierten Industrien hin zu technologieintensiveren Branchen hat in der österreichischen Industrie in der Vergangenheit auch tatsächlich stattgefunden (vgl. Abbildung 6-7). Die historische Erfahrung des letzten Vierteljahrhunderts zeigte jedoch auch, dass der Prozess der „schöpferischen Zerstörung“ nur langsam abläuft. Überdies wurde der Strukturwandel der jüngeren Vergangenheit weniger durch das Wachstum technologieintensiver als vielmehr durch die Anteilsverluste arbeitsintensiver Branchen wie der Textilindustrie bestimmt.

Außerdem wurde der intrasektorale Strukturwandel der Sachgüterproduktion während der letzten 25 Jahre von einem bedeutenderen, intersektoralen Strukturwandel überdeckt: Primärproduktion und Sachgüterproduktion verloren sukzessive Beschäftigungs- und Wertschöpfungsanteile an den Dienstleistungssektor. Größte Gewinner dieser Tertiarisierung sind wissensbasierte Dienstleistungen. Die Anteilsgewinne dieser Gruppe sind wesentlich auf ihre Verwendung als Vorleistungen in Industrie und Dienstleistungssektor zurückzuführen (vgl. Peneder, Kaniovski, Dachs 2001). Da Dienstleistungen im Allgemeinen eine niedrigere F\&EIntensität als die Industrie aufweisen, lässt die Teritarisierung die F\&E-Quote tendenziell fallen. Trotzdem kann nicht a priori von einer sinkenden Technologieintensität durch die Tertiarisierung gesprochen werden. Im Unterschied zu Hochtechnologiebranchen in der Sachgüterindustrie 
sind viele wissensbasierte Dienste durch einen intensiven Einsatz von Informations- und Kommunikationstechnologien und einen hohen Anteil an Mitarbeitern mit tertiärer Ausbildung geprägt.

Abbildung 6-7: Strukturwandel in der österreichischen Wirtschaft: Veränderung der Anteile an der Wertschöpfung, 1976 - 2000, laufende Preise
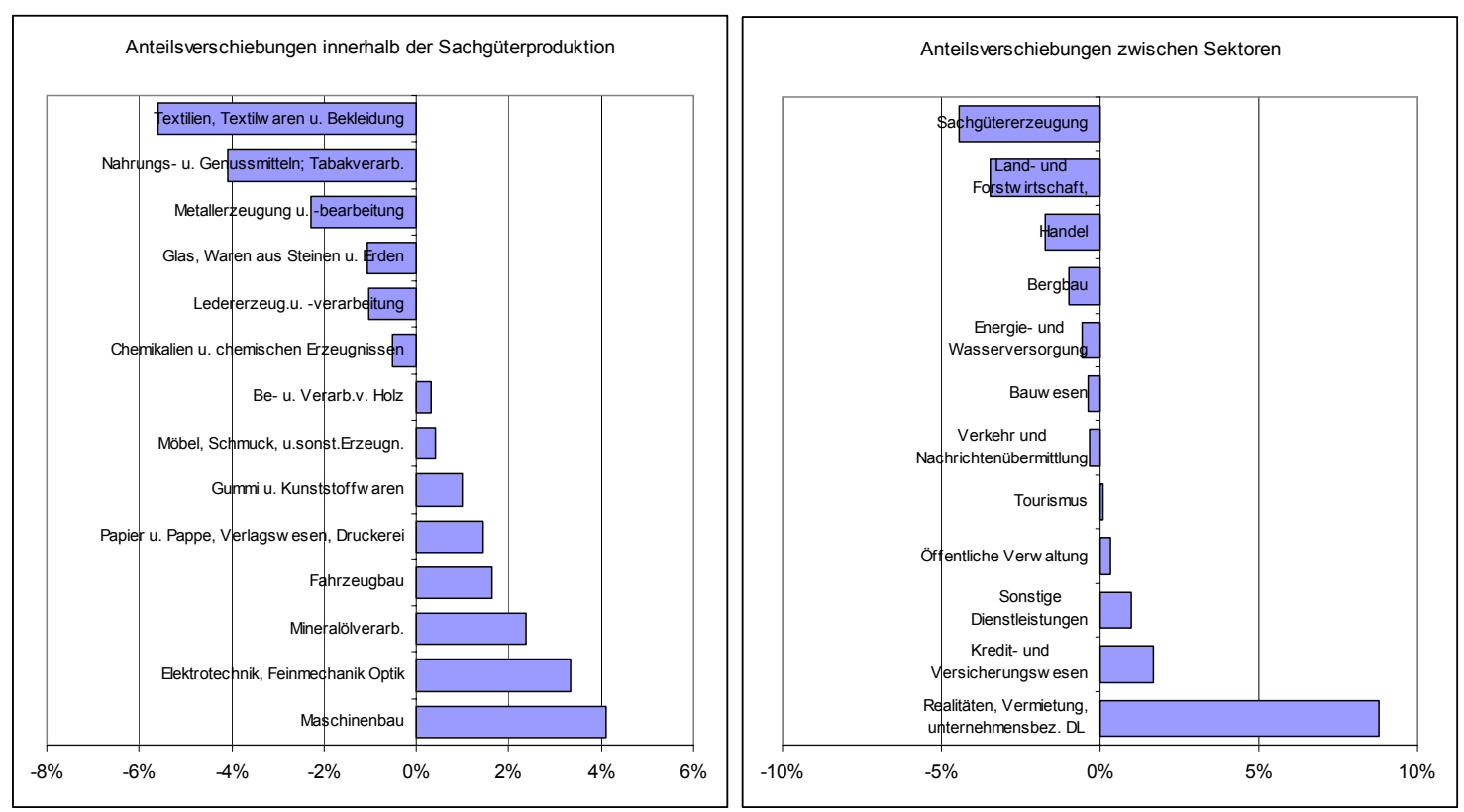

Quelle: Statistik Austria

Aus diesen Zusammenhängen ergeben sich zwei Schlüsse für die Technologiepolitik: Erstens muss sich Technologie- und Innovationspolitik der Bedeutung von strukturellen Einflussfaktoren bewusst sein. Hier stellt sich die grundsätzlichere Frage nach der Zweckmäßigkeit der einseitigen Fixierung auf die Ausgaben für Forschung und Entwicklung als zentralen Indikator für technologische Leistungsfähigkeit. Diese Haltung ist aus der Notwendigkeit der Politik, prägnant zu formulieren, verständlich; sie entspricht jedoch nicht dem derzeitigen Stand des innovationspolitischen Wissens. Besonders im Dienstleistungssektor, wo Innovationen im größeren Umfang auf externe Technologie, Humankapital und organisatorische Veränderungen basieren, sind eigene Ausgaben für Forschung und Entwicklung kein zentraler Input im Innovationsprozess.

Zweitens muss Technologie- und Innovationspolitik stärker als bisher den Konnex zu allgemeinen wirtschaftspolitischen Fragen, besonders den Themen Gründungsdynamik und Strukturwandel suchen. Am Beispiel Finnlands zeigt sich, dass eine hohe F\&E-Quote weniger Ursache als vielmehr Folge eines dynamischen Strukturwandels ist. Nokia allein investiert inzwischen etwa 30\% des finnischen GERD (vgl. Bellak 2002); Eine nachhaltige Steigerung der Technologieintensität der heimischen Wirtschaft lässt sich nur durch eine Veränderung der Branchenstruktur erreichen. Die Politik kann hier wichtige Impulse geben. 


\section{Informations- und Kommunikationstechnologien}

Zahlreiche seit Anfang der 90er Jahre entwickelte sozioökonomische Konzepte erklären den Strukturwandel in industrialisierten Volkswirtschaften mit Verweis auf die Bedeutung des Aufbaus und Austauschs von Information und Wissen. Vielfach rückt dabei der zunehmende Einsatz neuer und zu niedrigen Kosten verfügbarer Anwendungen von Informations- und Kommunikationstechnologien (IKT) in den Mittelpunkt des Interesses. Konzeptuelle Überlegungen wie z.B. zur Digitalen Ökonomie, Informationsgesellschaft oder Internetökonomie - heben die Bedeutung von IKT als in der Wirtschaft universell einsetzbare Querschnittstechnologien hervor. Das hohe, bislang nur teilweise erschlossene Innovationspotential beschränkt sich daher keineswegs auf die Entwicklung neuer Anwendungen und Dienste im IKT-Sektor. Vielmehr werden durch die konkrete Anwendung von IKT in de facto allen Wirtschaftsbereichen Innovationspotentiale freigelegt. Diffusion und Adoption von IKT finden konsequenterweise als wichtige Parameter zur Einschätzung der technologischen Leistungsfähigkeit von Volkswirtschaften Beachtung.

Das vorliegende Kapitel konzentriert sich deshalb auf aktuelle Trends der Diffusion von IKT im internationalen Vergleich sowie auf die Diffusion von business-to-business-Anwendungen, einer anwendungsorientierten Technologie mit besonderen kommerziellen Möglichkeiten.

\subsection{Diffusion und Adoption von IKT}

Für entwickelte Industrieländer lässt sich eine stark zunehmende Nutzung von IKT anhand zahlreicher Indikatoren feststellen. Auf aggregierter Ebene weist beispielsweise die IT-Intensität - gemessen an den IT-Ausgaben in Prozent des Bruttoinlandsprodukts - einen rapiden Anstieg während der zweiten Hälfte der 90er Jahre auf. Zumindest bis zum Jahre 2000 wurden selbst in fortgeschrittenen Ländern wie z.B. den USA keine Sättigungstendenzen erkennbar (vergleiche Abbildung 7-1). Der internationale Vergleich macht außerdem deutlich, dass die Mehrzahl der Europäischen Länder gegenüber den USA nach wie vor gravierende Rückstände aufweist; von einem erfolgreichen Aufholprozess kann somit lediglich in wenigen Ländern (insbesondere Portugal) gesprochen werden.

Gemessen an der IT-Intensität ist Europa durch ein regionales Nord-Süd-Gefälle gekennzeichnet. Neben den skandinavischen Ländern finden sich Großbritannien, die Schweiz und die Niederlande in der Spitzengruppe. Österreich liegt ähnlich wie die Bundesrepublik Deutschland, Belgien und Irland im Mittelfeld und somit leicht unter dem Vergleichswert für die gesamte Europäische Union. Die hohe Wachstumsdynamik ist in allen Ländern annähernd gleich stark ausgeprägt, sodass sich insbesondere zwischen 1997 und 2000 nur geringfügige Änderungen in der direkten Rangfolge ergeben haben. 
Abbildung 7-1: Entwicklung der IT-Intensität im internationalen Vergleich

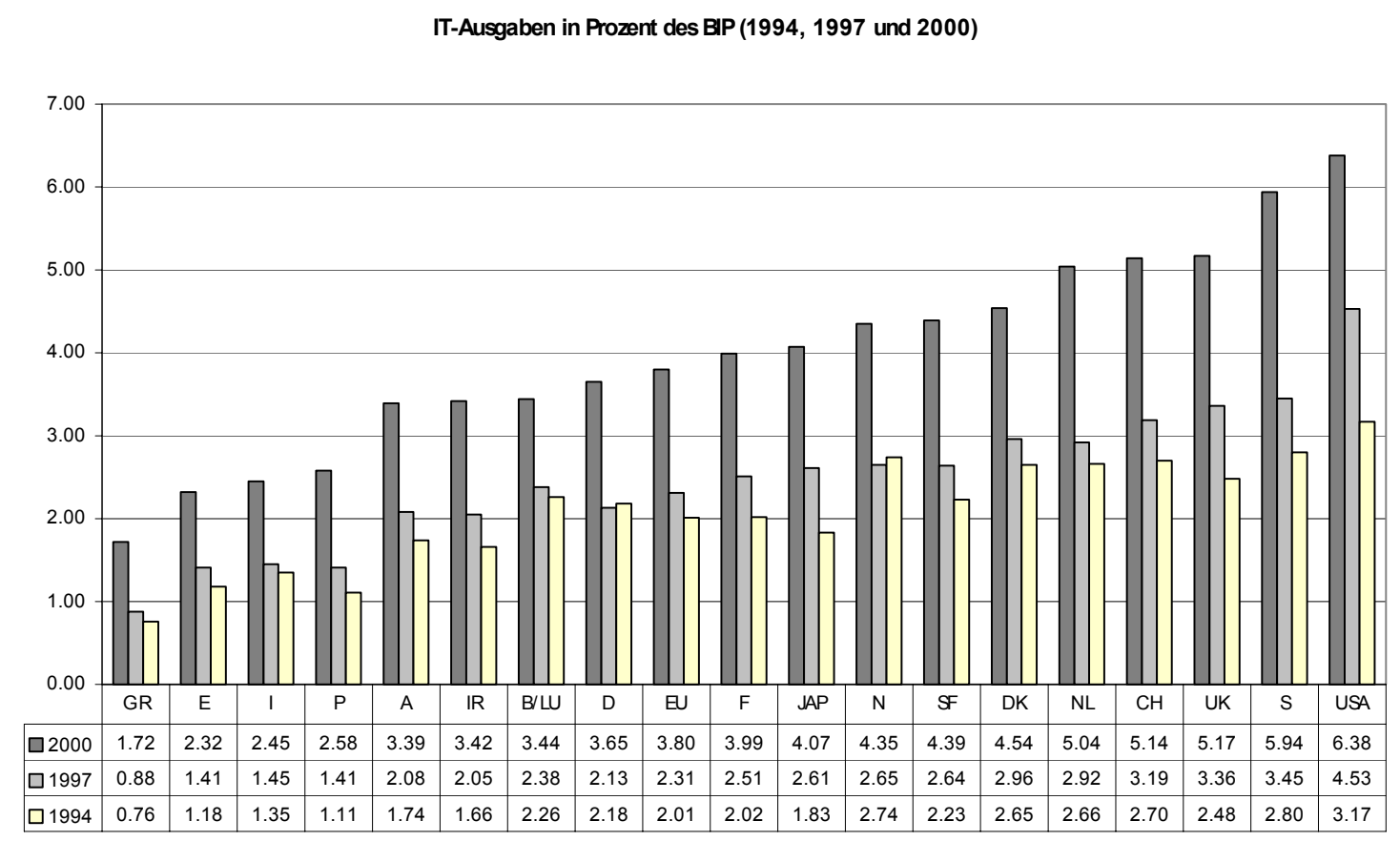

Quelle: EITO, eigene Berechnungen

Der Indikator IT-Intensität bildet lediglich einen ersten Anhaltspunkt für einen Vergleich der Diffusionsprozesse von IKT. Spezifika und Fortschritte der Länder lassen sich anhand der Nutzerdichte bei konkreten Anwendungen näher bestimmen. PC-Dichte (PCs pro 100 Einwohner), Internetnutzer-Dichte und Mobiltelefonnutzer-Dichte erlauben eine differenziertere Betrachtung. Alle drei Indikatoren haben im Zusammenhang mit Entwicklungen zur Informationsgesellschaft einen nicht vernachlässigbaren Stellenwert: Eine hohe Verbreitung von PCs bildet de facto eine grundlegende Voraussetzung für die Diffusion auch fortschrittlicher Anwendungen. Selbiges gilt mittlerweile auch für das Internet, das gegen Ende der 90er Jahre seinen Stellenwert als technologische Plattform für den Informationsaustausch deutlich erhöht hat. Schließlich hat auch die Mobilkommunikation als IKT-Anwendung an Bedeutung gewonnen, zumal sie einerseits in manchen Ländern bereits höhere Teilnehmerzahlen als das Festnetz aufweist, andererseits mobile Breitbandkommunikation (GPRS, UMTS) für die Zukunft wesentliche Verbesserungen der Telekommunikationsinfrastruktur erwarten lässt.

Bei Personalcomputern handelt es sich zwar um eine als weitgehend etabliert geltende Anwendung, erhebliche Zuwächse während der 90er Jahre sind dennoch festzustellen; zudem ist eine Sättigung auch in Ländern mit hohem Verbreitungsgrad nach wie vor nicht absehbar (vergleiche Abbildung 7-2). Ähnlich wie die IT-Intensität weist auch die Computer-Dichte für die meisten europäischen Länder erhebliche Rückstände gegenüber den USA nach. Trotz geringfügiger Verschiebungen im Länderranking wiederholt sich das Bild starker regionaler Disparitäten innerhalb Europas und eines Nord-Südgefälles. Österreich gehört zumindest bis zum Jahr 2000 zu jenen Ländern, die den Vergleichswert für die gesamte Europäische Union nicht erreichen. 
Abbildung 7-2: Computer-Dichte im internationalen Vergleich

PCs pro 100 Enwohner (1994, 1997 und 2000)

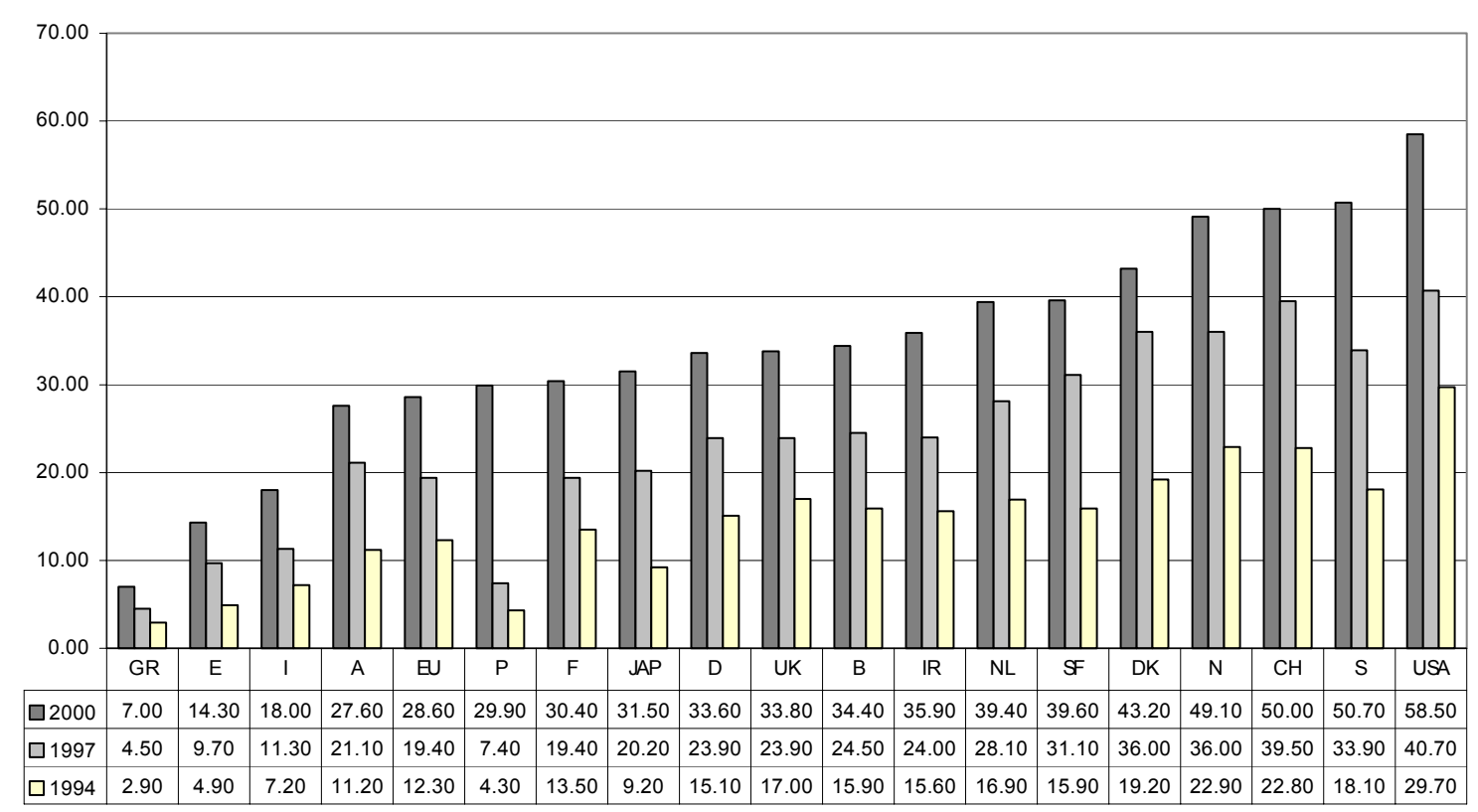

Quelle: ITU-Datenbank, eigene Berechnungen

Im Gegensatz zum PC, dessen Diffusion sich durch kontinuierliches Wachstum während der beiden letzten Dekaden auszeichnet, weist die Verbreitung des Internet eine weitaus höhere Mitte der 90er Jahre einsetzende - Dynamik aus (vergleiche Abbildung 7-3). Bei der Internetnutzer-Dichte (gemessen an Internetnutzern pro 100 Einwohnern) übertreffen die skandinavischen Länder selbst die USA. Das Nord-Süd-Gefälle innerhalb der Europäischen Union bleibt erhalten.

Österreich nimmt in der Internetnutzung eine erstaunlich gute Position über dem europäischen Vergleichswert ein. Neuere internationale Erhebungen zur Nutzung des Internet in privaten Haushalten (z.B. Flash Eurobarometer 112, November 2001) lassen sich als Indiz für gelungene Aufholprozesse während der beiden letzten Jahre interpretieren. Beispielsweise geben $48 \%$ der befragten Österreicher an, Zugang zum Internet zu haben (EU15: 38\%). Die Rückstände gegenüber den drei führenden Ländern Norwegen (64\%), Schweden (61\%) und Dänemark (59\%) sind nach wie vor erheblich; allerdings sind die Unterschiede zu den anderen Ländern der Spitzengruppe - Finnland (50\%), Großbritannien (49\%) und Irland (48\%) vernachlässigbar gering.

Als Indizien für insgesamt gute Voraussetzungen der IKT-Diffusion im privaten Bereich in Österreich sprechen auch andere Ergebnisse des Flash Eurobarometer 112:

- Die Internetzugänge österreichischer Haushalte sind überdurchschnittlich breitbandig: Überdurchschnittlich viele heimische Internetnutzer verwenden als Zugangstechnologie ISDN 
(24,4\%), ein Kabelmodem (22,1\%) und ADSL (7,1\%); die Vergleichswerte für die EU15 liegen bei $16,0 \%, 9,1 \%$ bzw. $6,3 \%$.

- Hoher Anteil von tatsächlichen Internet-Nutzern: Rund 62,1\% der befragten Österreicher nutzen das Internet auch tatsächlich (EU15: 47,9\%). Höhere Anteile an Internet-Nutzern finden sich lediglich in Dänemark (70,3\%), den Niederlanden $(68,6 \%)$, Schweden $(67,9 \%)$ und Finnland $(63,5 \%)$.

- Hoher Anteil von Vielnutzern: Innerhalb der Europäischen Union zeichnen sich die heimischen Internetnutzer mit rund $77 \%$ den höchsten Anteil von Vielnutzern - Nutzung erfolgt täglich oder zumindest mehrmals die Woche - aus.

- Hohe Verfügbarkeit und Nutzung von Internetzugängen am Arbeitsplatz: Die Nutzung des Internet von Zuhause liegt in Österreich mit $68,6 \%$ lediglich im europäischen Durchschnitt. Demgegenüber ist der Zugang zum Internet vom Arbeitsplatz mit 50,9\% nur in Schweden $(53,2 \%)$ und Finnland $(52,3 \%)$ höher, somit auch weit über dem europäischen Vergleichswert $(40,1 \%)$.

\section{Abbildung 7-3: Internetnutzer-Dichte im internationalen Vergleich}

Internetnutzer pro 100 Enwohner (1994, 1997 und 2000)

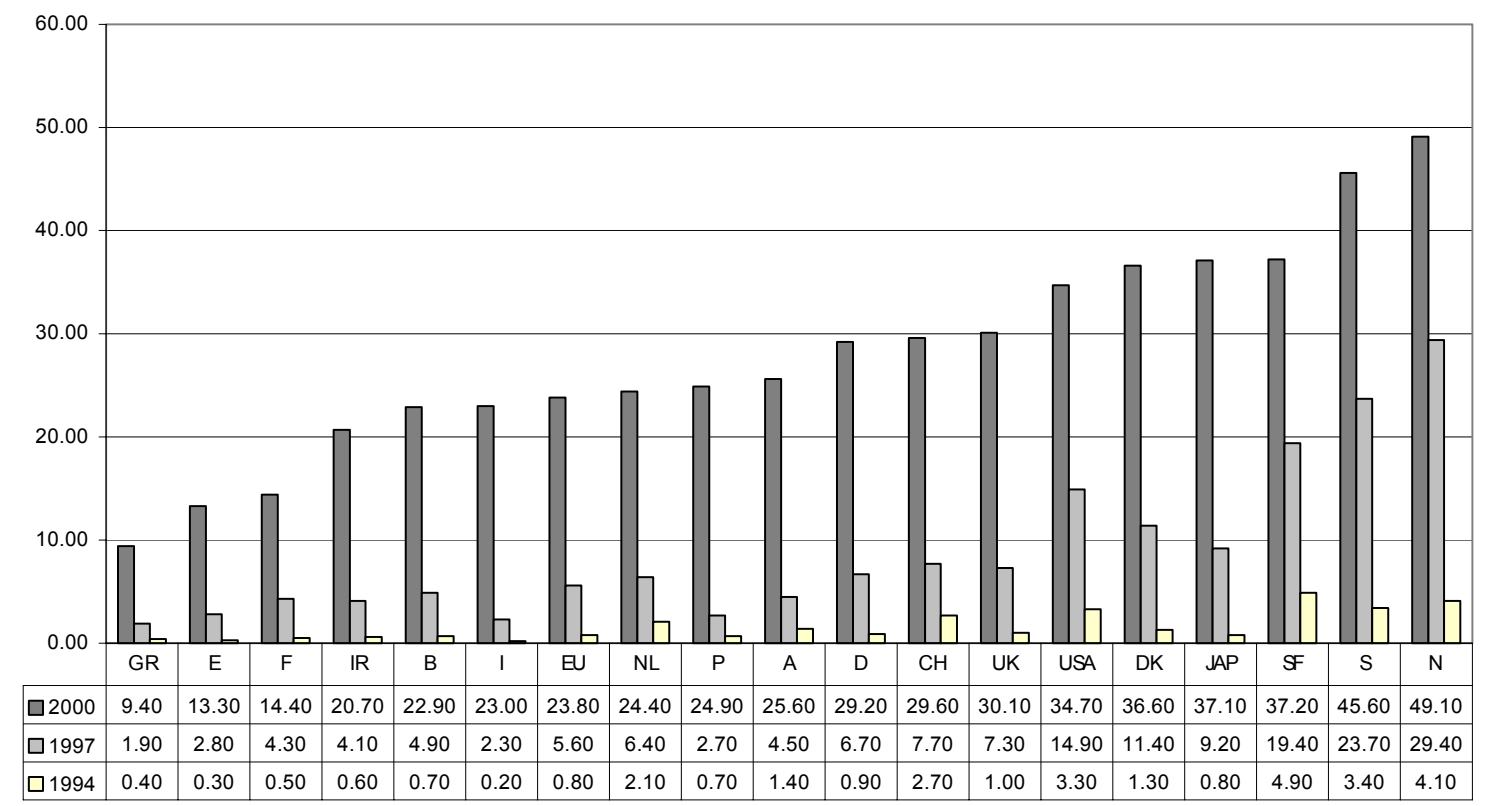

Quelle: ITU-Datenbank, eigene Berechnungen

Die Diffusion von IKT weist ein Nord-Süd-Gefälle und andere starke Unterschiede innerhalb Europas und zwischen Europa und den USA auf. Österreich hat seine Position in den letzten Jahren deutlich verbessert, gehört aber noch bei einigen Indikatoren zur Gruppe der Länder, die unter dem EU-Durchschnitt liegen. 
Die europäischen Umfragen des Eurobarometer zeigen einerseits, dass das Internet als eine der zentralen IKT-Anwendungen eine sehr dynamische Entwicklung aufweist. Andererseits lassen sich auch Rückschlüsse zu Aufholprozessen in Österreich ziehen. Die Position Österreichs innerhalb Europas hat sich während der letzten Jahre deutlich verbessert und ein Aufschließen zu den skandinavischen Ländern erscheint in Reichweite. Inwieweit Österreich im Trend zu breitbandigen Zugangstechnologien eine überdurchschnittliche Entwicklung beibehalten kann, bleibt offen, zumal Kabel-TV-Netze eine starke Nutzung erfahren, ADSL hingegen als Infrastruktur eine bislang untergeordnete Rolle spielt.

\section{Abbildung 7-4: Mobiltelefon-Dichte im internationalen Vergleich}

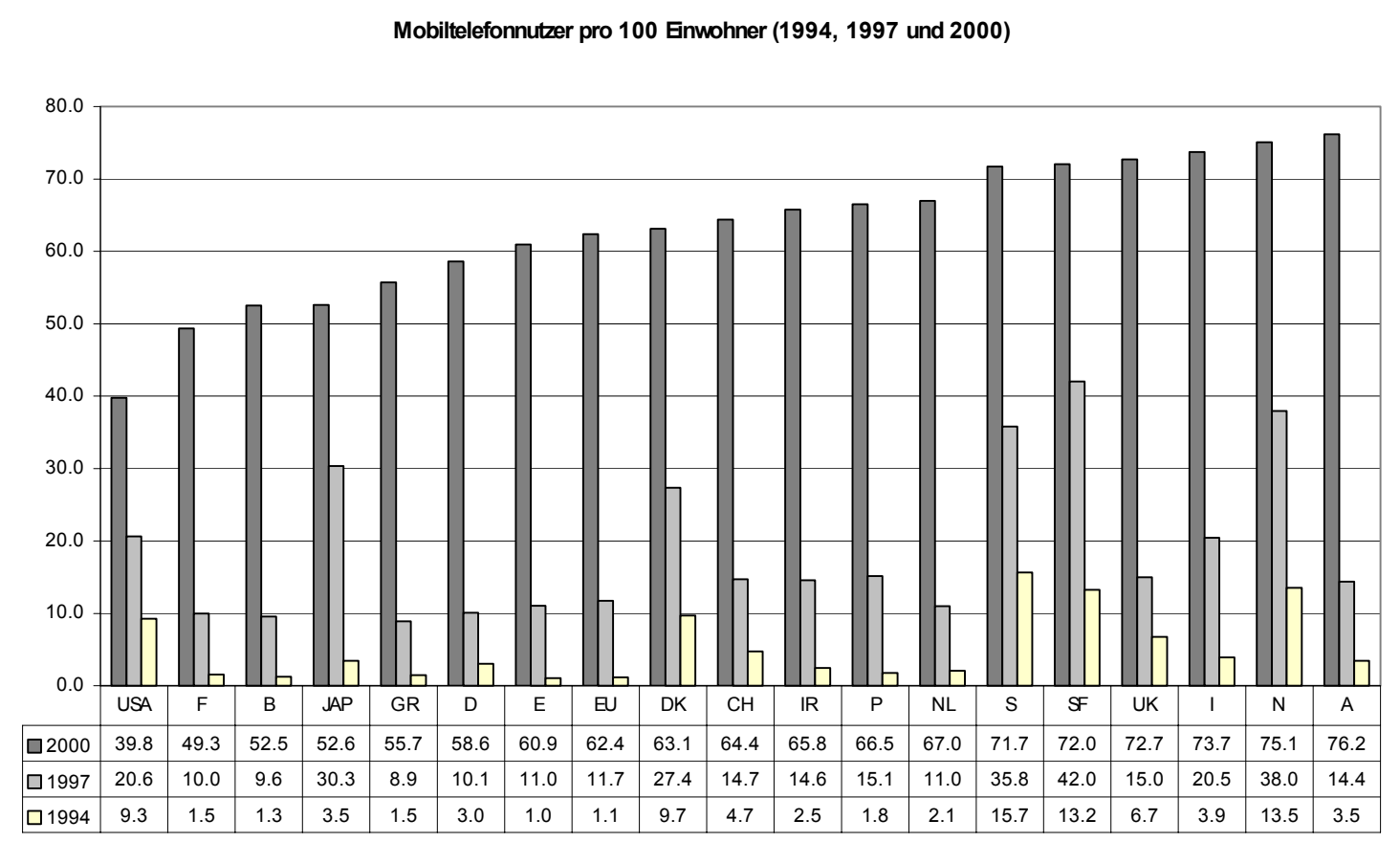

Quelle: ITU-Datenbank, eigene Berechnungen

Eine für Europa insgesamt positive Entwicklung zeigt sich auch im Bereich der Mobilkommunikation, die während der letzten Jahre einerseits als Anwendung von IKT, andererseits als Infrastruktur an Bedeutung gewonnen hat. In mehreren europäischen Ländern - so auch in Österreich - finden sich in mobilen Netzen bereits mehr Nutzer als im Festnetz. Darüber hinaus zeigt sich bereits vor der Verfügbarkeit von UMTS, dass mobile Netze zunehmend zur Datenkommunikation (beispielsweise als Internetzugang) Verwendung finden. Daten der International Telecommunications Union (ITU) zeigen außerdem, dass die europäischen Länder wesentlich stärkere Diffusionsraten verzeichnen als die USA (vergleiche Abbildung 7-4). Österreich hat in diesem Bereich eine besonders dynamische Entwicklung durchgemacht und erreichte im Jahr 2000 eine Spitzenposition. 


\subsection{Die Diffusion von IKT und e-Commerce im Unternehmenssektor}

Während eine unverminderte Diffusion von Internet und Mobiltelefonen zu beobachten ist, hat die "harte Landung" der New Economy über den IKT-produzierenden Sektor hinaus in zahlreichen Branchen Wirkungen gezeigt. Viele Unternehmen kürzten als eine der ersten Maßnahmen in der Rezession ihre Investitionen in Netzwerke, Server und Applikationen, zahlreiche Unternehmen wurden insolvent oder mussten sich nach dem Verfall ihrer Aktienkurse und dem Ausbleiben von neuem Kapital grundlegend umorientieren.

An der Einschätzung der positiven Effekte von IKT in langfristiger Perspektive hat sich hingegen wenig geändert. Überzogene Erwartungen wie etwa die Spekulationen um ein Verschwinden von Konjunkturzyklen sind hier einer realistischeren Sicht der New Economy „beyond the hype“ (vgl. OECD 2001a) gewichen. Besonders hoffnungsvoll scheint hier der Bereich des elektronischen Geschäftsverkehrs zwischen Unternehmen (b2b). Die größten Produktivitätsgewinne sind jedoch weniger in Unternehmen der "dot.com"-Branchen als vielmehr in bestehenden Wirtschaftsbereichen zu erwarten, wo e-commerce-Anwendungen erfolgreich in bestehende Geschäftsprozesse eingebaut wurden. So hat sich herausgestellt, dass traditionelle Versandhäuser gegenüber vielen neuen e-commerce-Unternehmen im Vorteil sind, da sie über eine entwickelte Logistik und Lagerhaltung verfügen. E-commerce ist für diese Unternehmen eine weitere Vertriebsschiene, die sich in die bestehenden Absatzkanäle integriert.

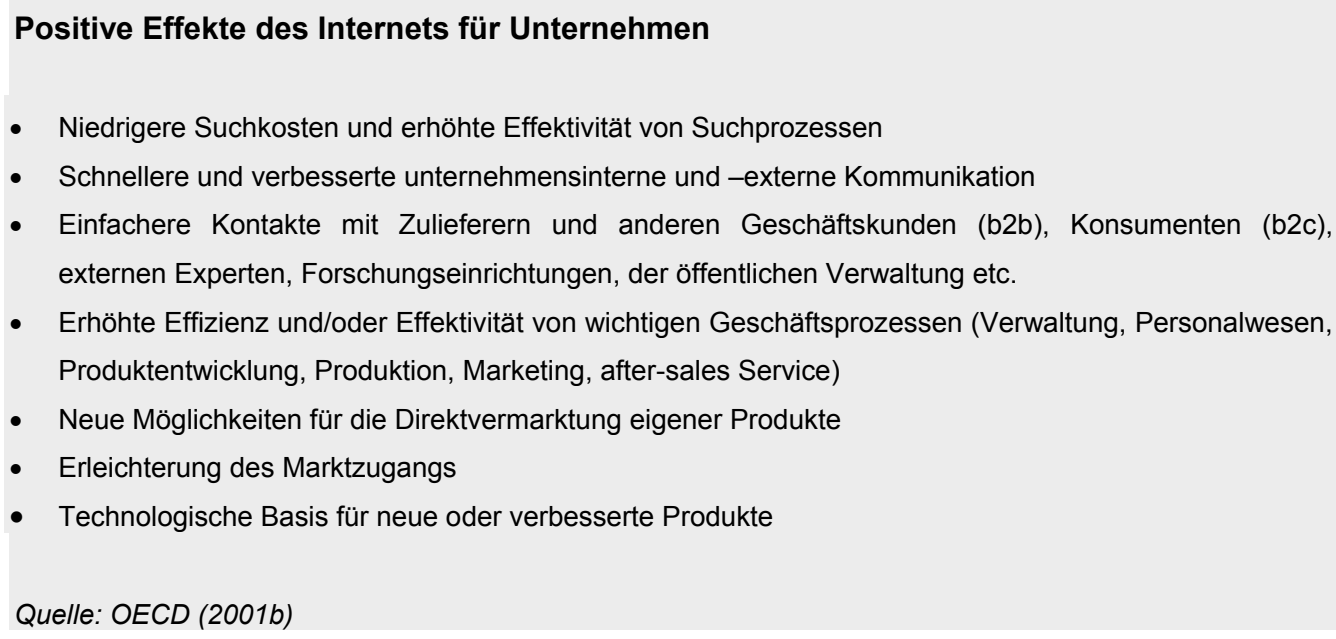

Voraussetzung für die Nutzung von b2b ist das Vorhandensein einer adäquaten betrieblichen Infrastruktur. Abbildung 7-5 zeigt die zeitliche Diffusionskurve für die PC-Nutzung und für das Vorhandensein einer eigenen Homepage. Eine eigene Homepage weist auf eine gewisse Vertrautheit mit dem Medium „Internet“ hin und kann somit als Indikator für die prinzipielle Bereitschaft für b2b-Techniken interpretiert werden kann. Differenziert wird dabei zwischen drei Unternehmensgrößenklassen: (i) kleine Unternehmen mit bis zu 25, (ii) mittlere Unternehmen mit 26 bis 100 und (iii) große Unternehmen mit über 100 Beschäftigten. 
Hinsichtlich der PC-Nutzung kann der Diffusionsprozess als abgeschlossen betrachtet werden, wobei bereits in den 80er Jahren die höchsten Wachstumsraten der PC-Nutzung erzielt wurden. Allerdings sagt dieser einfache Indikator noch nichts über die Qualität der Nutzung (i.S. der Ausschöpfung der technischen und organisatorischen Potenziale dieser Technik) aus. Diese Einschränkung trifft ebenfalls auf die PC-Dichte innerhalb der Unternehmen (z.B. Anteil der Beschäftigten mit PC-Zugang) zu. Zwischen den drei Beschäftigungsgrößenklassen zeigen die Diffusionskurven eine zeitliche Verschiebung der Durchdringungsquote von jeweils etwa zwei bis drei Jahre in den 80er Jahren, während es im Zuge der 90er Jahre zu einer deutlichen Konvergenz kam und die Unterschiede zwischen den Größenklassen mittlerweile gänzlich verschwunden sind.

Bei Internetauftritten kam es ab Mitte der 90er Jahre zu einem drastischen Wachstum. Binnen sechs Jahre erhöhte sich der Anteil der Unternehmen mit eigener Homepage von ca. $10 \%$ auf über $80 \%$ (Großunternehmen) bzw. über $70 \%$ (Mittelunternehmen) sowie etwas über $60 \%$ (Kleinunternehmen). Diese rasche Diffusion zeigt die Anpassungs- bzw. Adoptionsfähigkeit österreichischer Unternehmen. Insgesamt kann gefolgert werden, dass in einem Großteil der österreichischen Unternehmen (und zwar weitgehend unabhängig von der Größenklasse) wichtige technische und organisatorische Voraussetzungen für die prinzipielle Einsatzmöglichkeit von B2B-Techniken gegeben sind.

Abbildung 7-5: Diffusion von IKT-Technologien in österreichischen Unternehmen (in \%)
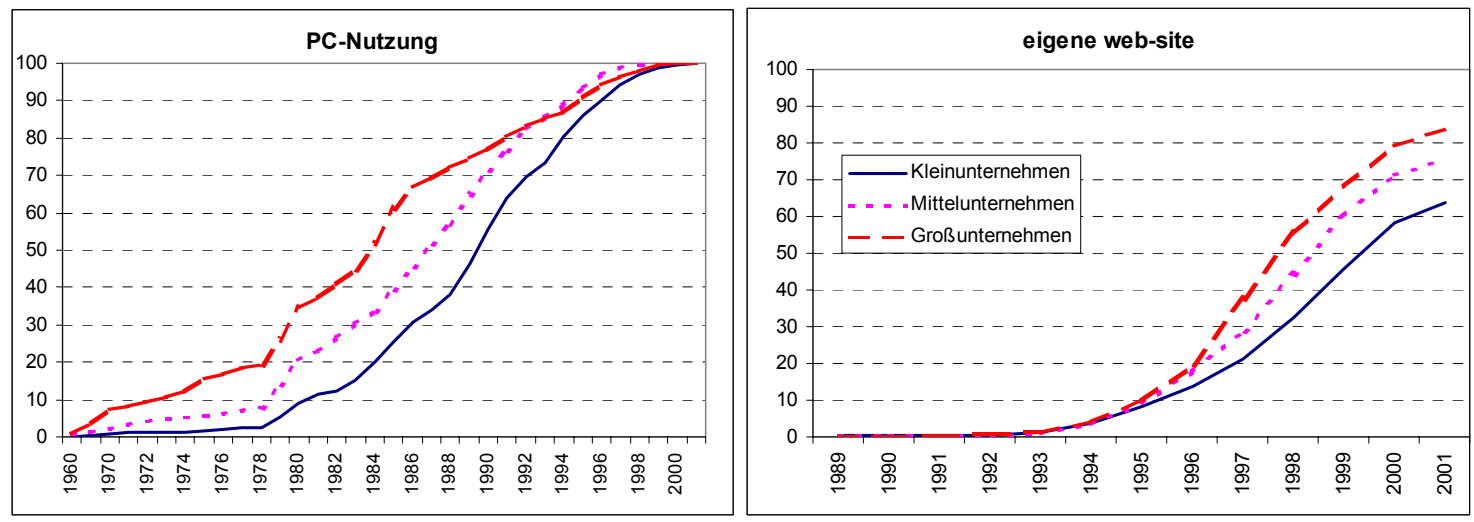

Quelle: Joanneum Research

Während also die Nutzungsdichte von PCs bereits de facto $100 \%$ erreicht hat, ist der Verbreitungsgrad von b2b-Anwendungen derzeit noch wesentlich geringer. Im Jänner 2001 haben nach Statistik Austria (2001) 15\% der Unternehmen Waren und Dienstleistungen über Netzwerke (Internet und EDI ${ }^{24}$ ) gekauft bzw. 12\% verkauft. Die Ergebnisse erlauben aufgrund der breiten Stichprobe erstmals eine Hochrechnung auf die gesamte österreichische Wirtschaft.

24 Electronic Data Interchange: Ein System zum Datenaustausch zwischen verschiedenen Geschäftspartnern. Ein Beispiel sind die Reservierungssysteme im Flugverkehr. Im Unterschied zu manchen Internet-Plattformen, die prinzipiell von jedem Unternehmen genutzt werden können, sind EDI-Systeme jedoch im Regelfall geschlossene, proprietäre Systeme mit einem begrenzten, fest vorgegebenen Benutzerkreis. 
Es ergeben sich für das Jahr 2000 e-commerce-Verkäufe im Wert von 8,21 Mrd. $€$ bzw. ecommerce-Einkäufe im Wert von 7,95 Mrd. €. Insgesamt betrug der Anteil der Umsätze, die über e-commerce abgewickelt wurden, im Jahre 2000 2,5\%. Der Anteil der Verkäufe an Endkonsumenten ist derzeit noch gering.

Nutzer von e-commerce sind im Dienstleistungssektor generell häufiger als in der Sachgüterproduktion zu finden. Besonders in der Datenverarbeitung, der Nachrichtenübermittlung und im Hotel- und Gastgewerbe sind einschlägige Anwendungen weit verbreitet. Dabei muss jedoch genau unterschieden werden, ob von Ver- oder Einkäufen gesprochen wird. Die Wahrscheinlichkeit, dass e-commerce verwendet wird, steigt sowohl auf Käufer- als auch auf Verkäuferseite mit der Unternehmensgröße stetig an (vgl. Abbildung 7-6).

\section{Abbildung 7-6: Einsatz von elektronischen Netzwerken für Ein- und Verkäufe von Unternehmen, Jänner 2001}
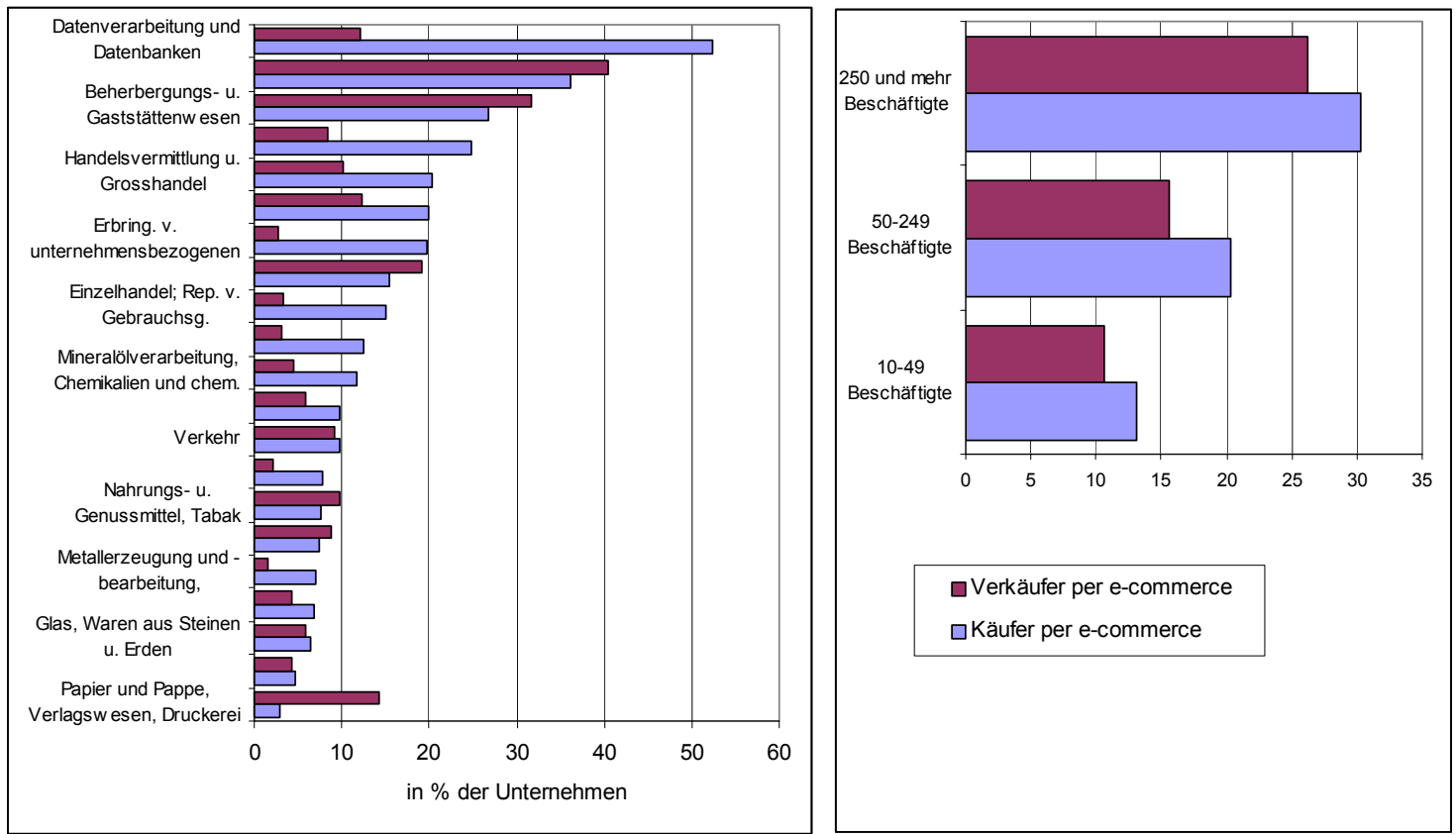

Quelle: Statistik Austria

Der Anteil der Unternehmen, die b2b-Technologien ${ }^{25}$ nutzen, ist also im Vergleich zur de facto flächendeckenden Verbreitung der dafür notwendigen technischen Infrastruktur vergleichsweise gering. Relativ häufig werden - vor allem von Kleinunternehmen - Internetshops im Zuge des Beschaffungswesens genutzt. Die Teilnahme an e-Marktplätzen (= Handelsplattformen im Internet) sowie die Teilnahme an elektronischen Ausschreibungen folgen an zweiter bzw. dritter Stelle, wobei sich diesbezüglich eine deutliche Abhängigkeit von der Unternehmensgröße zeigt.

Herkömmliche, bereits „klassische“ IKT-Technologien wie e-mail-Verkehr wurden ebenfalls abgefragt, werden hier aber nicht unter dem Sammelbegriff „B2B-Techniken“ subsummiert. Der Anteil der Nutzer von e-mail mit Geschäftspartnern beträgt für alle drei Größenklassen mehr als $80 \%$. 
Größere Unternehmen (jeweils ca. $25 \%$ ) nutzen diese Techniken eher als kleine Unternehmen (ca. $13 \%$ ). Von (noch) geringer Bedeutung sind Internet-Auktionen wie auch (geschlossene) elektronische Einkaufsverbünde. Bei der Nutzung von EDI zeigt sich, dass zumindest einige b2b-Techniken EDI, was die Nutzungshäufigkeit betrifft, abgelöst haben. Ausnahme hierzu sind allerdings vor allem im Vertriebswesen bei Großunternehmen zu beobachten, wo EDI noch deutlich vor den "neuen“ internet-gestützten b2b-Techniken genutzt wird. Der Einsatz in diesem Umfeld bringt es mit sich, dass EDI zwar von der Zahl der Nutzer inzwischen relativ unbedeutend ist, vom Volumen der Transaktionen jedoch noch immer zu den bedeutendsten Technologien zählt (vgl. Statistik Austria 2001).

\section{Abbildung 7-7: Nutzung von b2b-Technologien in österreichischen Unternehmen (in \%)}
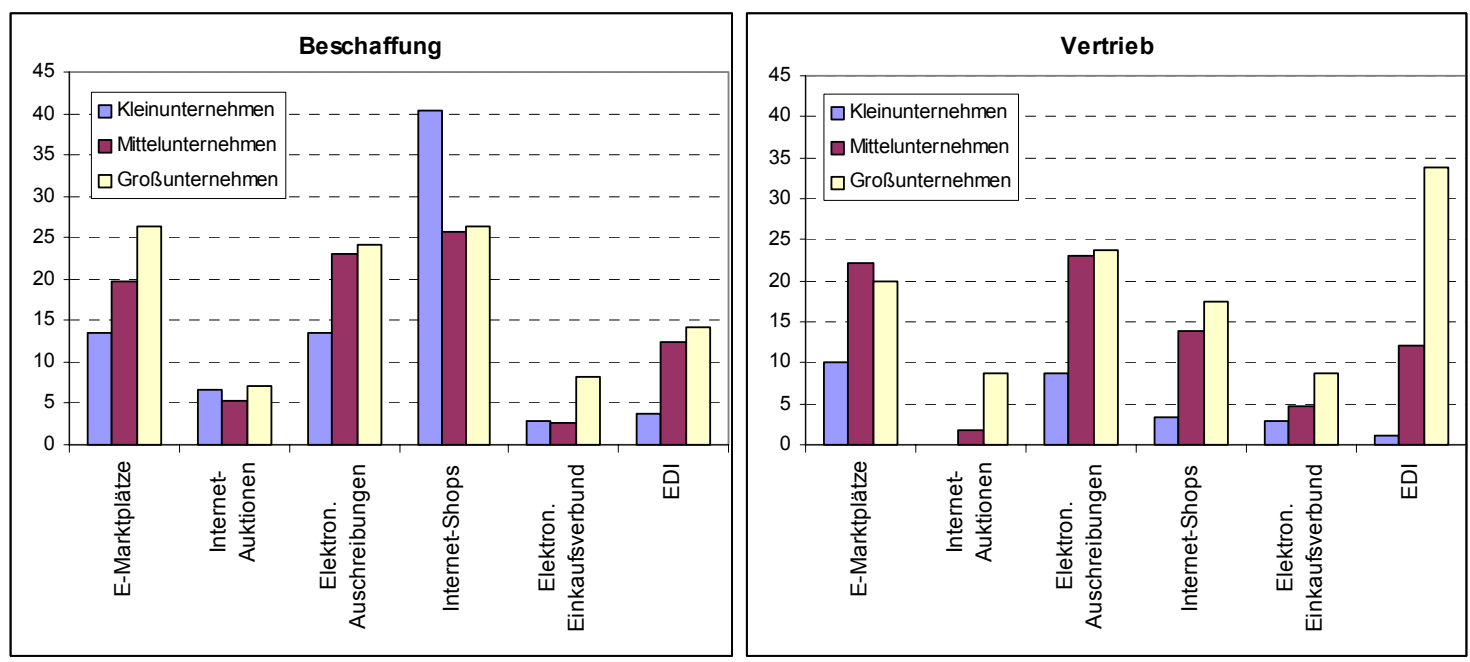

Quelle: Joanneum Research

Die Gründe für die starken Unterschiede in der Nutzung dieser Technologien zwischen verschiedenen Branchen und Größenklassen sind vielfältig. Wie bei jeder Innovation ist der Grad der Einführung stark von unterschiedlichen technologischen Möglichkeiten, Marktchancen und anderen branchenspezifischen Gegebenheiten geprägt. Nicht alle Güter eignen sich gleichermaßen für den Verkauf über das Internet. Ein hoher Grad an Standardisierung und die exakte Beschreibbarkeit des Gutes, wie etwa bei elektronischen Komponenten, Chemikalien oder Flugtickets, sind wichtige Vorbedingungen.

Obwohl bereits viele Unternehmen über die technologischen Voraussetzungen für b2bAnwendungen verfügen, ist der Anteil der Benutzer noch gering. Großunternehmen scheinen bei dieser Technologie Vorteile zu haben, da ihre Nutzungsintensität höher ist.

Auch kann in Branchen, die bereits jetzt besonders IKT-intensiv sind, mit einer höheren Bereitschaft zum Einsatz von e-commerce gerechnet werden, da sowohl Fachkräfte als auch eine leistungsfähige Infrastruktur verfügbar und bereits Erfahrungen mit dem Einsatz von IKT vorhanden sind. Beispiele dafür sind die Datenverarbeitung und die Nachrichtenübermittlung. 
Wenn bereits EDI-Anwendungen im Einsatz sind, wie etwa im Handel oder in der Automobilindustrie, könnte dies eine Erleichterung für die Beteiligung an b2b-Systemen sein, besonders wenn diese branchenweit ältere EDI-Systeme ersetzen (vgl. Boston Consulting Group 2000). Andererseits ist aber auch vorstellbar, dass ein vorhandenes, gut funktionierendes EDI-System auch ein Hindernis für die Einführung von b2b-Anwendungen sein kann und die Unternehmen damit in eine lock-in Situation geraten.

Ein eindeutiges Ergebnis vieler Untersuchungen ist, dass die Nutzungsrate von b2b in größeren Unternehmen höher ist. Dies lässt sich wohl damit begründen, dass große Unternehmen die Risken einer frühen Einführung leichter tragen können als Kleinunternehmen. Allerdings hat sich die Hypothese, dass größere Firmen Vorteile im Innovationsprozess haben, nicht immer als korrekt herausgestellt (vgl. Cohen 1995). Gerade unter den Kleinunternehmen ist eine Reihe von Firmen tätig, die ohne Internet gar nicht gegründet worden wären.

\section{Abbildung 7-8: Einschätzung der künftigen Entwicklung des b2b-Aufkommens}
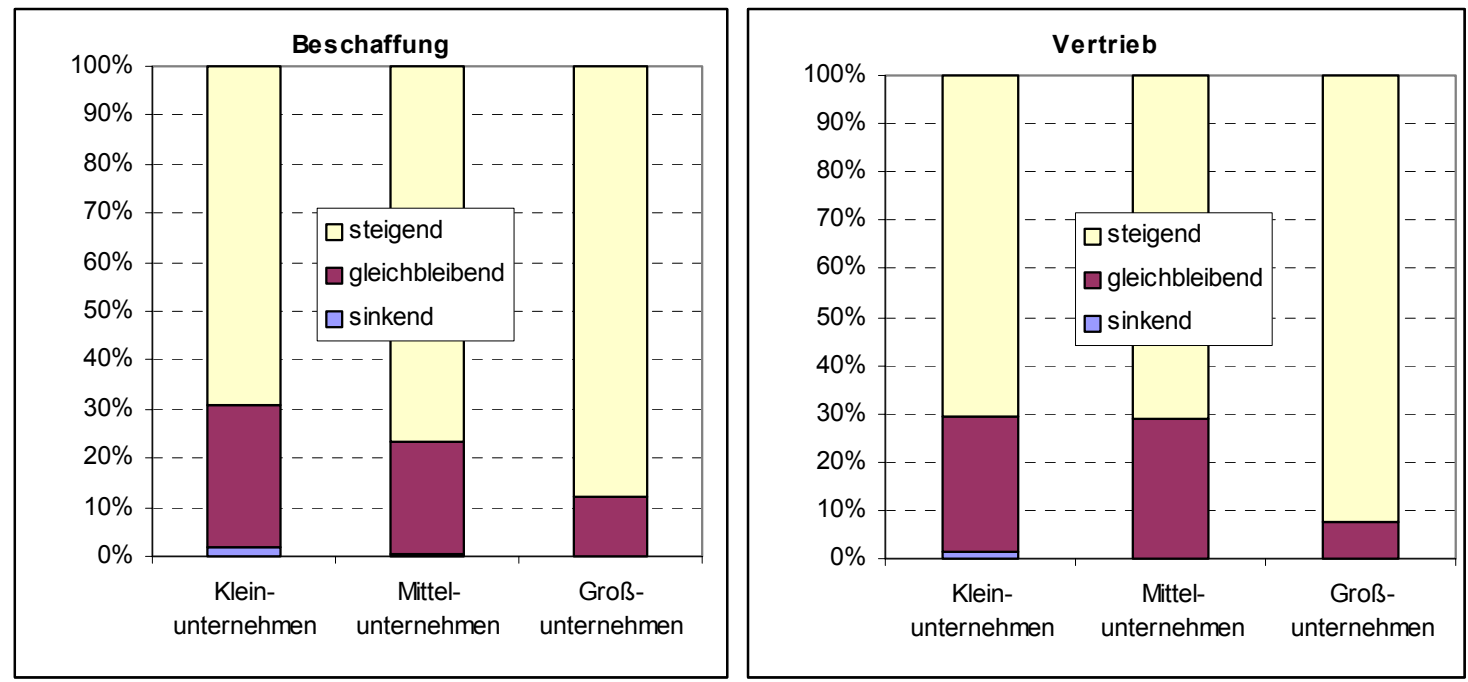

Quelle: Joanneum Research

Bertschek und Fryges (2002) weisen außerdem auf einen Netzwerk- oder „Bandwagon-Effekt“ in der Diffusion von b2b-e-commerce hin: Der Nutzen (und damit die Wahrscheinlichkeit der Einführung) einer bestimmten Innovation steigt für das innovierende Unternehmen mit der Zahl der übrigen Nutzer dieser Innovation. Daraus ergibt sich ein positiver Feedback- oder Netzwerkeffekt (vgl. Shapiro, Varian 1999, S. 173 f.). Etablierte Systeme mit vielen Benutzern, also vielen potentiellen Geschäftspartnern, sind attraktiver als neue Systeme, auch wenn diese in technologischer Hinsicht den etablierten Lösungen überlegen sind. Auch in Branchen, in denen bereits viele Benutzer b2b einsetzen, können solche Effekte entstehen, wobei aber auch Faktoren wie die Marktmacht großer Abnehmer, die ein einheitliches System für ihre Lieferanten durchsetzen, eine Rolle spielen können.

Die überwältigende Mehrheit der Unternehmen geht für die nächsten Jahre von einem steigenden Aufkommen elektronisch getätigten Geschäftsverkehrs aus. Die nicht unbe- 
trächtlichen Unterschiede in der Einschätzung zwischen den Größenklassen der Unternehmen deuten darauf hin, dass auch die Diffusion dieser Techniken ähnlich phasenverschoben ablaufen wird, wie oben für PC-Bestand bzw. für das Vorhandensein eines eigenen Internetauftritts gezeigt wurde.

Zusammenfassend kann gesagt werden, dass sich die österreichischen Unternehmen infrastrukturell (gemessen an den einfachen Indikatoren PC-Nutzung und Web-Seite) bereits durchaus an die technologische Entwicklung angepasst haben. Auch wenn internationale Vergleichsdaten fehlen, zeigen die Nutzungsanteile der diversen b2b-Technologien, dass hinsichtlich einer avancierteren Nutzung der Möglichkeiten der IKT - vor allem bei kleineren Unternehmen - noch eine gewisse „Reserviertheit" vorhanden ist. Angesichts eines funktionierenden Marktes für b2b-Lösungen und den beträchtlichen ökonomischen Anreizen, die für die Adaption dieser Technologie existieren, scheint hier jedoch kein unmittelbarer technologiepolitischer Handlungsbedarf gegeben. Informationsdefizite und mentale Reservationen gegenüber der neuen Technologie könnten im Rahmen bestehender Interessensvereinigungen, etwa des Automobilclusters, besser überwunden werden.

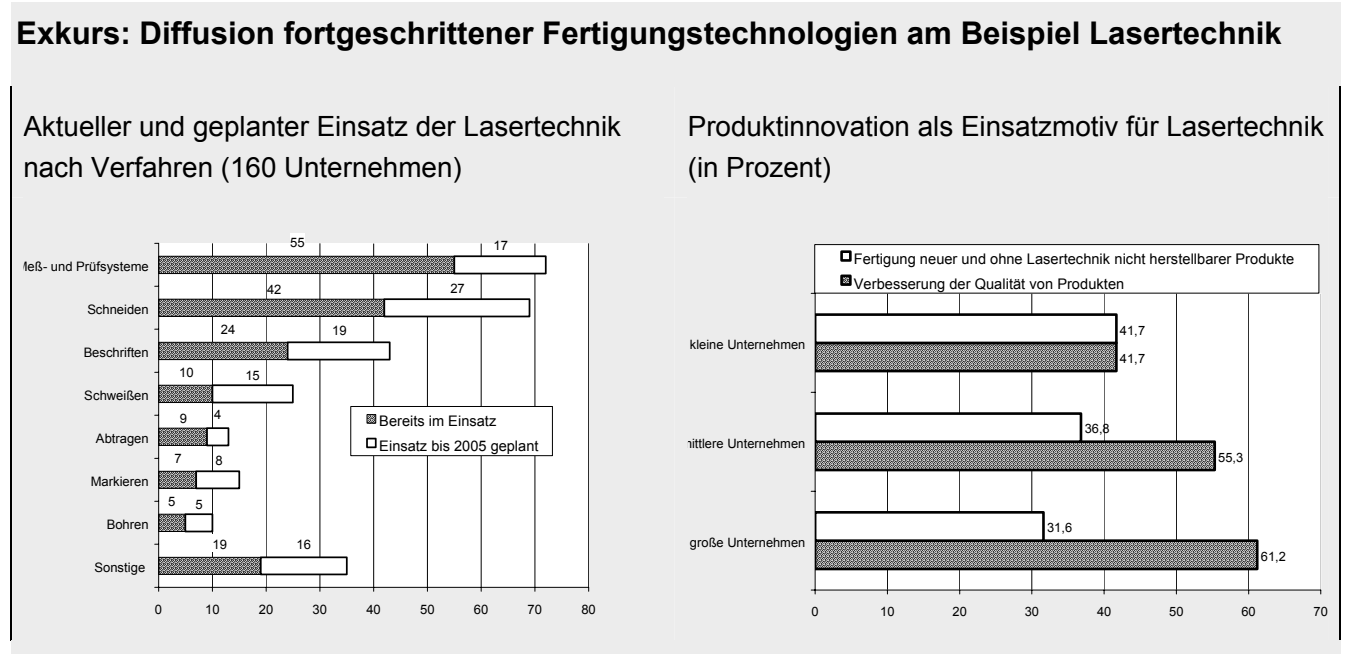

Quelle: Knoll 2001

Die Lasertechnik ist ähnlich wie das Internet eine Querschnittstechnologie, die sich durch hohes Entwicklungspotential, Anwendbarkeit in (fast) allen Wirtschaftsbereichen, breite Nutzungsvielfalt sowie ein komplementäres Verhältnis zu anderen Technologien auszeichnet. In den 90er Jahren haben Trends industrieller Produktionstechnik wie z.B. die Einführung flexibler Fertigungskonzepte und Miniaturisierung von Produkten eine rasche Diffusion lasertechnischer Verfahren der Messtechnik und der Materialbearbeitung begünstigt.

Die im Auftrag des BMVIT durchgeführte Unternehmensbefragung "LASER 2000+" (Knoll 2001) bestätigt einerseits die zunehmende Adoption laserbasierter Verfahren durch heimische Unternehmen während der letzten 20 Jahre; andererseits lassen sich Diffusionsunterschiede in Abhängigkeit von Branchenzugehörigkeit und Unternehmensgröße festmachen, die auch mittelfristig - d.h. bis 2005 - weitgehend bestehen bleiben: Bei zunehmender Vielfalt unter kommerziellen Bedingungen einsetzbarer Verfahren werden auch in naher Zukunft Vorreiterbranchen wie z.B. Elektronik, Metallbearbeitung, Fahrzeug- und Maschinenbau überdurchschnittliche Anwenderquoten aufweisen; eine starke Abhängigkeit der Entscheidung über den Lasertechnikeinsatz von der Unternehmensgröße bleibt ebenfalls erhalten. Darüber 
hinaus lässt sich neben einer wachsenden Zahl von Anwendern ein Trend zum gleichzeitigen Einsatz verschiedener Verfahren festmachen.

Dass der Lasertechnikeinsatz maßgeblich zur Erhöhung der Wirtschaftlichkeit für einzelne Fertigungsschritte beiträgt, ist vor allem unter bestehenden Anwendern weitgehend unbestritten. Bei den Einsatzmotiven stehen die Optimierung von Prozessparametern bzw. Besonderheiten der Verfahren - wie z.B. Präzision, Wiederholgenauigkeit und Automatisierbarkeit - im Vordergrund.

Das innovative Potential der Lasertechnik beschränkt sich allerdings nicht auf Prozessinnovationen. So stellen - insbesondere mit zunehmender Unternehmensgröße Qualitätsverbesserungen der Produkte, die mit Einschränkungen als inkrementelle Produktinnovationen zu werten sind, ein wichtiges Motiv für den Einsatz lasergestützter Fertigungsverfahren dar. Beachtlich ist aber auch, dass mehr als ein Drittel der befragten Anwender den Lasertechnik-Einsatz mit den Fertigungserfordernissen von Produktinnovationen begründet. Vor allem kleinere und mittlere Unternehmen halten den Einsatz lasertechnischer Verfahren oft für eine unumgängliche Voraussetzung zur Herstellung neuer Produkte.

\subsection{Die österreichische Politik zur Informationsgesellschaft}

Die österreichischen Unternehmen und Haushalte haben, wie gezeigt wurde, große Fortschritte auf dem Weg in die Informationsgesellschaft gemacht. Nun gilt es, sich auf die Entwicklung und Verbreitung von Anwendungen zu konzentrieren, die die kommunikativen Möglichkeiten des Internets voll nutzen. Die Vermittlung der notwendigen Kenntnisse im Umgang mit neuen Technologien bleibt eine weitere wichtige Aufgabe.

Die österreichischen Anstrengungen sind dabei eng mit der „eEurope“-Initiative ${ }^{26}$ der Europäischen Kommission verknüpft, die sich das Ziel gesetzt hat, die Vorteile von Informations- und Kommunikationstechnologien für Europa so weit als möglich zu nutzen. Die österreichische IKT-Politik auf Bundes- und Länderebene hat sich in den letzten Jahren neben e-government (siehe unten) im wesentlichen auf drei Bereiche konzentriert (vgl. Dachs, Wagner 2001):

- Angebotsseitig werden durch die Förderung der Entwicklung neuer IK-Technologien, etwa im Rahmen der Kompetenzzentrenprogramme oder durch den FFF, Impulse gesetzt. Neue Programme, wie etwa FIT-IT, ein Impulsprogramm des BMVIT, das sich zunächst auf den Themenbereich "embedded systems" konzentriert, oder eine IKT-Gründungsinitiative des BMWA, sind in Vorbereitung.

- Nachfrageseitige Politik konzentriert sich neben der öffentlichen Beschaffung vor allem auf die Vermittlung von Kenntnissen und Fertigkeiten im Umgang mit IKT. Voraussetzung war hier die Ausstattung von Schulen mit PCs und leistungsfähigen Internet-Zugängen, die inzwischen für mittlere und höhere Schulen weitestgehend abgeschlossen ist $^{27}$.

\footnotetext{
26 http://www.europa.eu.int/information_society/index_de.htm

${ }^{27} \mathrm{http}: / / \mathrm{www}$. bmbwk.gv.at/start.asp?bereich=3\&OID=5814\&/1=17\&/2=65\&/3=5816
} 
Schwerpunkt zukünftiger Aktivitäten wird die Lehrerfortbildung und Erwachsenenbildung sein.

- Ein weiteres wesentliches Element der österreichischen IKT-Politik ist die Gestaltung der Rahmenbedingungen für den Einsatz von IKT. Beispiele sind die Tätigkeit der Regulierungsbehörde RTR oder die Anpassung von Rechtsvorschriften an die Erfordernisse von e-commerce (z. B. im Verbraucherrecht, Signaturgesetz) und egovernment (z. B. Verwaltungsverfahren).

Trotz einer sichtbaren Verstärkung der Initiativen während der letzten Jahre bleiben im Vergleich mit anderen Ländern einige Schwachstellen festzustellen. So scheinen die Aktivitäten verschiedener Ministerien im Bereich IKT vielfach noch zu wenig koordiniert. Vorbilder in anderen Ländern zeigen jedoch (z. B. die Niederlande, vgl. Dachs, Knoll 2001), dass gerade ein Bereich wie IKT, der inzwischen die Tätigkeit aller Ministerien betrifft, erhöhten Abstimmungsbedarf hat. Durch die Schaffung des IKT-Boards, dem die IT-Verantwortlichen aller Ressorts angehören, und des Chief Information Office ${ }^{28}$ als zentrale Stabstelle des Bundes für die IKTStrategie wurde zunächst für den Einsatz von IKT in der Verwaltung ein erster Schritt für mehr Koordination gesetzt.

Zweitens fehlt noch immer eine strategische „Vision“ der IKT-Politik, die zwar angekündigt, aber noch nicht veröffentlicht wurde. Ein solches Dokument könnte einerseits die nationale Politik im Kontext der europäischen Initiativen festlegen, andererseits die Integration zwischen IKT-Politik und der allgemeinen Forschungs- und Technologiepolitik verstärken.

Während der letzen Jahre hat sich vor allem e-government, also der Kontakt zwischen Bürgerln und Verwaltung über elektronische Medien, als wichtiger Schwerpunkt der österreichischen IKT-Aktivitäten etabliert. Darunter fallen sowohl Informationsdienste (z. B. help@gv.at) als auch Anwendungen, die Transaktionen zwischen Verwaltung und Bürgern erlauben. Ein attraktives Angebot an öffentlichen Dienstleistungen im Internet - von der Gewerbeanmeldung über Steuerangelegenheiten bis zu Sozialverwaltung - kann zu einer wesentlichen Verwaltungsvereinfachung und damit höherer Servicequalität für Private und Unternehmen führen. Darüber hinaus können Bund, Länder und Gemeinden durch die Gestaltung ihrer Dienste aber auch wesentlichen Einfluss auf die Nutzungsintensität von IKT nehmen.

Während die infrastrukturellen Voraussetzungen wie Personal Computer und Netzwerke in beinahe allen österreichischen Unternehmen und vielen Haushalten vorhanden sind, befindet sich die Verbreitung der entsprechenden Verwaltungsanwendungen in Österreich und auch europaweit erst im Anfangsstadium. Die e-government-Strategie der österreichischen Bundesregierung setzt deshalb zum einen auf die Automatisierung und Standardisierung der internen Verwaltungsabläufe sowie auf die Einrichtung von Schnittstellen zwischen einzelnen Dienststellen. Ein wichtiges Projekt in diesem Zusammenhang ist die Entwicklung des elektronischen Aktes $^{29}$ (elak). Elak soll bis 2003 umgesetzt werden und den Papierakt als

\footnotetext{
${ }^{28} \mathrm{http}: / /$ www.cio.gv.at/

${ }^{29}$ http://www.cio.gv.at/
} 
Original ablösen. Auch der Verkehr zwischen Ressorts soll weitgehend auf elektronischem Wege stattfinden.

Eine zweite wichtige Voraussetzung für Transaktionsdienste ist die Fähigkeit des/der Bürger/ln, sich auf elektronischem Weg eindeutig identifizieren zu können, beispielsweise einen Antrag in einem Verwaltungsverfahren rechtsgültig über Internet zu signieren. Nur so können auch auf elektronischem Weg rechtsverbindliche Willensäußerungen gesetzt werden. Diese Anforderungen soll nach dem Plan der Bundesregierung eine Bürgerkarte ${ }^{30}$ erfüllen. Die Bürgerkarte wird mit einer elektronischen Signatur ausgestattet und mit entsprechenden Verzeichnisdiensten der Verwaltung kompatibel sein. Als Bürgerkarten soll sich jede Chipkarte eignen, die Anforderungen der elektronischen Signatur erfüllen kann. Als eine der ersten Karten soll die elektronische Sozialversicherungskarte ${ }^{31}$, die 2003 eingeführt wird, mit den Funktionalität der Bürgerkarte ausgestattet werden können.

\footnotetext{
${ }^{30} \mathrm{http}: / /$ www.buergerkarte.at

${ }^{31} \mathrm{http}: / / \mathrm{www}$.hauptverband.at/chipkarte/index.htm
} 


\section{Biotechnologie in Österreich}

\subsection{Einführung}

Die Biotechnologie ist eine Querschnittstechnologie, auf deren Basis sich eine rasch wachsende Zahl von Anwendungen mit hoher Wertschöpfung in verschiedenen Branchen entwickelt hat. Biotechnologie wird entsprechend ihrer Anwendungen in "rote" (Pharma-), "grüne“ (Agro-), und "graue" (Umwelt-) Biotechnologie eingeteilt. Während es bei der grünen Biotechnologie gerade in Europa noch Akzeptanzprobleme gibt, ist die rote Biotechnologie bereits fest etabliert. So ist das weltweite Marktvolumen für rekombinante Pharmazeutika in einem Jahrzehnt von 3 Mrd. \$ (1990) auf 42,5 Mrd. \$ (2000) gewachsen. Ihr Anteil am gesamten Pharmamarkt ist im entsprechenden Zeitraum von 2,5\% auf $17 \%$ gestiegen. (Informationssekretariat Biotechnologie 2001). Selbst in der Entwicklung von traditionellen, nicht auf gentechnologischem Weg hergestellten Medikamenten sind biotechnologische Verfahren nicht mehr wegzudenken. So können z.B. Zellrezeptoren gentechnisch erzeugt werden, mit denen "konventionelle" Substanzen auf deren Wirksamkeit untersucht werden können. Biotechnologie findet auch immer weitere Einsatzmöglichkeiten, etwa in der Chemie und Kunststoffindustrie, weshalb die Biotechnologie für viele Marktbeobachter als Schlüsselindustrie des 21. Jahrhunderts gilt. Schätzungen zufolge werden im Jahre 2010 in der Biotechnologie 1,2 Mio. Fachkräfte eine Wertschöpfung von über 400 Mrd. € erwirtschaften (BCG 2001).

In den USA sind die ersten Biotechnologiefirmen bereits Mitte der 70er Jahre entstanden. Anfang der 80er Jahre gab es dort die ersten Börsengänge von Biotechnologiefirmen (Genentech 1980, Amgen 1983). Gegenüber Europa ist der Vorsprung amerikanischer Biotechnologiefirmen beträchtlich, was sich auch an der Marktkapitalisierung zeigt. So sind alle europäischen, an der Börse gelisteten Unternehmen zusammen kaum mehr wert als das amerikanische Biotechnologieunternehmen Amgen alleine (Ernst \&Young 2001) ${ }^{32}$.

Europa scheint in letzter Zeit allerdings einen Teil des Rückstandes aufzuholen und überflügelt die USA seit einiger Zeit in den Wachstumsraten. Vor allem Deutschland hat mit Hilfe groß angelegter Programme in diesem Bereich eine dynamische Entwicklung eingeleitet und ist mit über 500 Biotechnologieunternehmen mittlerweile der bevorzugte Standort in Europa. Alleine im Großraum München haben sich inzwischen 117 Firmen angesiedelt. Österreich rangiert mit 11 Firmen dagegen auf einem der letzten Plätze in Europa (Tabelle 8-1). Selbst bei pro-KopfZahlen belegt Österreich mit Italien und Spanien die hintersten Plätze. Der Grund für die schlechte Position ist, dass sich Ansätze einer kommerziellen Biotechnologieszene in Österreich erst sehr spät entwickelten Österreich und einen Entwicklungsrückstand gegenüber den USA von ca. 20 Jahren hat.

32 Die Marktkapitalisierung von Amgen beträgt 63 Mrd. \$. (Kurs vom 14.3.02). 
Tabelle 8-1: Anzahl von Biotechnologiefirmen in Europa (Dezember 2000)

\begin{tabular}{lcc}
\hline Land & Biotechnologiefirmen & Firmen/ 1.000.000 Einwohner \\
\hline Deutschland & 504 & 5,88 \\
UK & 448 & 7,56 \\
Frankreich & 342 & 5,81 \\
Schweden & 235 & 25,96 \\
Schweiz & 93 & 12,61 \\
Niederlande & 79 & 4,97 \\
Italien & 64 & 1,11 \\
Belgien & 55 & 5,35 \\
Finnland & 53 & 10,36 \\
Dänemark & 51 & 9,59 \\
Irland & 39 & 11,17 \\
Norwegen & 37 & 8,29 \\
Spanien & 32 & 0,81 \\
\hline Österreich & 11 & 1,35 \\
\hline
\end{tabular}

Quelle: Allansdottir et al (2002).

Im Folgenden werden mögliche Einflussgrößen untersucht, die für eine prosperierende BiotechIndustrie verantwortlich sind.

\subsection{Wissenschafts- und Technologiebasis}

Ein wesentlicher Faktor für eine funktionierende Biotechnologieindustrie ist die wissenschaftliche Basis. Laut einer IHS-Studie (Baier, Griessler, Martinsen 2000) sind in Österreich 35 Forschungsinstitute tätig, davon sind 31 Universitätsinstitute. Die meisten Forschungsinstitute sind in Wien angesiedelt (17), es folgen Innsbruck (8), Graz (6), Niederösterreich (3) und Salzburg (1). Insgesamt arbeiten ca. 2.000 ForscherInnen in diesem Sektor, die meisten davon im Biopharmabereich (557).

Der Forschungsoutput kann durch die Anzahl von Fachpublikationen gemessen werden. Wie bereits in Kap. 4, Wissenschaftssystem, gezeigt wurde, nehmen die Publikationen in den Bereichen Lebenswissenschaften, also Immunologie und andere medizinische Fächer, Mikrobiologie oder Pharmakologie relativ zu anderen Wissenschaftsdisziplinen einen hohen Stellenwert ein. In absoluten Zahlen wurden in Österreich im Jahr 2001 ca. 3.000 wissenschaftliche Publikationen in den Lebenswissenschaften veröffentlicht. Dies entspricht einem Weltmarktanteil von $0,6 \%$ (Tabelle 8-2).

Gemessen an der Landesgröße ist der Wert durchaus beachtlich, andere Länder vergleichbarer Größe (Schweiz, Schweden, Dänemark, Finnland) liegen allerdings deutlich vor Österreich. Innerhalb Österreichs ist Wien ist bei weitem das größte Zentrum medizinischer und pharmazeutischer Forschung. 58\% aller Publikationen kommen von dort, dahinter folgen Innsbruck (21\%), Graz (16\%), und Salzburg (3\%). Wie in Kap. 4 ebenfalls gezeigt wurde, haben österreichische Publikationen in den Bereichen Pharmazie und Molekularbiologie außerdem einen überdurchschnittlichen Impact Factor. 
Tabelle 8-2: Veröffentlichungen von wissenschaftlichen Aufsätzen 2001 im medizinisch-pharmazeutischen Bereich

\begin{tabular}{|c|c|c|c|c|}
\hline Land & Publikationen & Weltmarktanteil & Einwohner & $\begin{array}{l}\text { Publikationen/ } \\
\text { 1.000 Einwohner }\end{array}$ \\
\hline USA & 139.081 & $28,99 \%$ & 276,6 & 0,50 \\
\hline Japan & 33.422 & $6,97 \%$ & 127,2 & 0,26 \\
\hline UK & 26.018 & $5,42 \%$ & 59,9 & 0,43 \\
\hline Deutschland & 24.252 & $5,05 \%$ & 82,1 & 0,30 \\
\hline Frankreich & 16.334 & $3,40 \%$ & 60,8 & 0,27 \\
\hline Italien & 14.830 & $3,09 \%$ & 57,7 & 0,26 \\
\hline Kanada & 13.531 & $2,82 \%$ & 30,5 & 0,44 \\
\hline Spanien & 8.894 & $1,85 \%$ & 39,5 & 0,23 \\
\hline Australien & 8.747 & $1,82 \%$ & 18,8 & 0,47 \\
\hline Niederlande & 8.435 & $1,76 \%$ & 16,0 & 0,53 \\
\hline China & 7.265 & $1,51 \%$ & 1.260 & 0,01 \\
\hline Schweden & 6.848 & $1,43 \%$ & 8,9 & 0,77 \\
\hline Schweiz & 4.979 & $1,04 \%$ & 7,2 & 0,69 \\
\hline Indien & 4.720 & $0,98 \%$ & 1.000 & 0,00 \\
\hline Korea & 4.389 & $0,91 \%$ & 46,5 & 0,09 \\
\hline Finnland & 3.365 & $0,7 \%$ & 5,2 & 0,64 \\
\hline Dänemark & 3.357 & $0,7 \%$ & 5,3 & 0,63 \\
\hline Österreich & 2.921 & $0,6 \%$ & 8,1 & 0,37 \\
\hline Welt & 479.788 & & 6.171 & 0,08 \\
\hline
\end{tabular}

Quelle: Public Medline, eigene Berechnungen

Die wissenschaftliche Basis ist eine notwendige, aber keine hinreichende Vorraussetzung für den Erfolg eines Clusters. Entscheidend ist die schnelle Umsetzung wissenschaftlicher Erkenntnisse in marktfähige Produkte. Ein beliebter Indikator für Produktinnovationen sind Patente. Hier stellt sich ein durchschnittliches Spezialisierungsmuster Österreichs bezüglich der Biotechnologie heraus. Der Anteil biotechnologischer Patente an allen österreichischen Patenten liegt mit 3,4\% deutlich vor Ländern wie Finnland und der Schweiz, überraschend auch vor den USA (Abbildung ). Dies ist ein Indiz dafür, dass Österreich im Biotechnologiebereich inzwischen seine wissenschaftlichen Stärken auch in kommerzielle Anwendungen übersetzen kann, und zwar wesentlich besser als in anderen Hochtechnologiesektoren wie der Mikroelektronik oder der Telekommunikation. Es liegt die Vermutung nahe, dass diese relativ günstige Position auch das Ergebnis der Forschungstätigkeit US-amerikanischer Konzerne in Österreich ist. 
Abbildung 8-1: Patentanmeldungen nach Technologiefeldern am US-Patentamt, 19952000

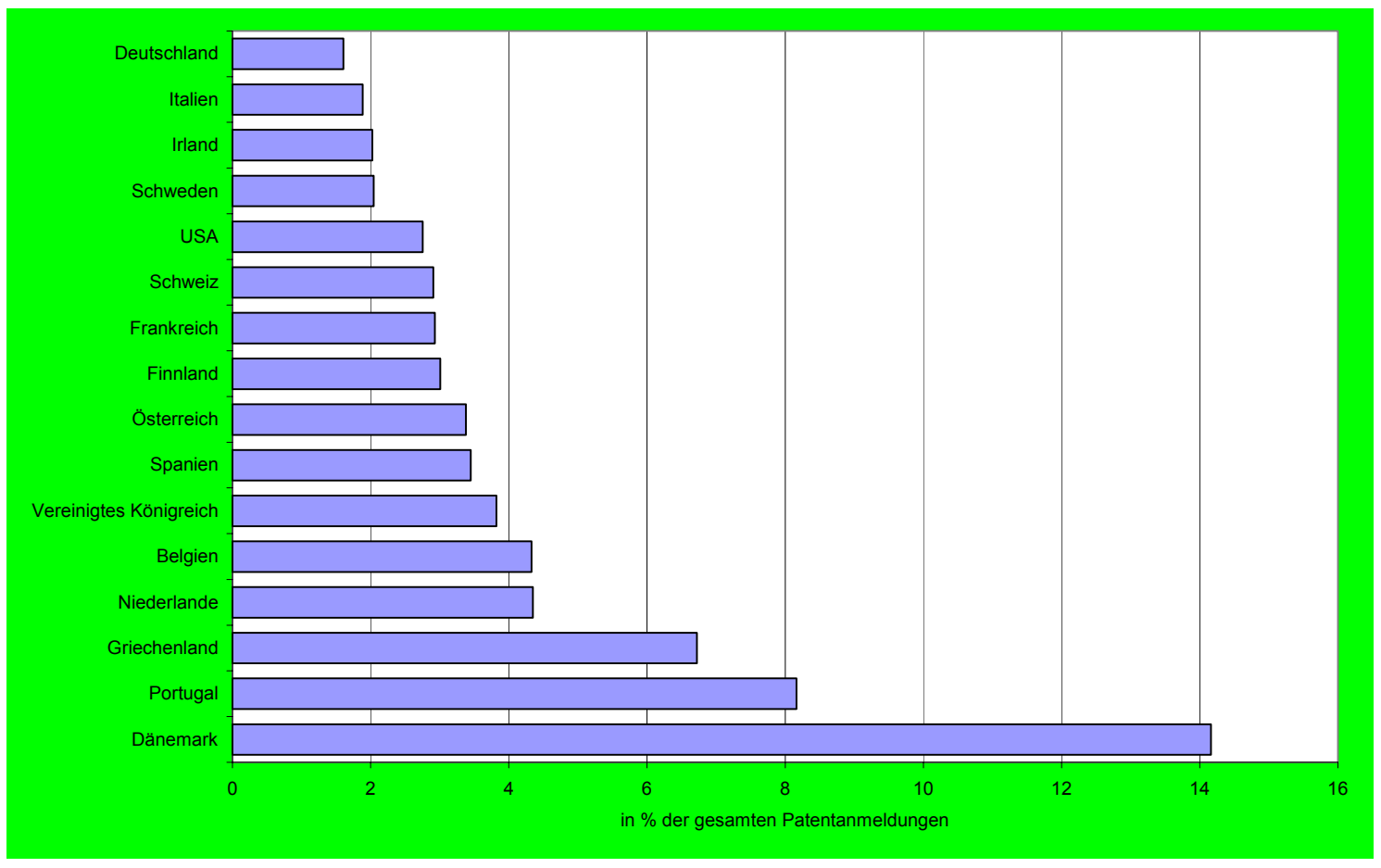

Quelle: CHI Research, eigene Berechnungen

Wird die Patenttätigkeit regional aufgeschlüsselt, so kann allein die Region Ostösterreich mit der Stadt Wien in der europäischen Spitze mithalten. Zwischen 1987 und 1996 kommt der Wiener Raum auf einen Marktanteil von 2,1 \% und liegt damit auf Platz 13 in Europa (Tabelle 8$3)$.

Tabelle 8-3: TOP 20 Biotechnologie Cluster in Europa (gemessen am Anteil europäischer Patente 1987-1996)

\begin{tabular}{llc|llc}
\hline Rang & Region & $\begin{array}{c}\text { Anteil } \\
\text { technologie in \% }\end{array}$ & $\begin{array}{c}\text { Bio- } \\
\text { teng }\end{array}$ & Region & $\begin{array}{c}\text { Anteil } \\
\text { technologie in \% }\end{array}$ \\
\hline 1 & Südost England (UK) & 8,6 & 11 & Niedersachsen (D) & 2,8 \\
2 & lle de France (F) & 7,3 & 12 & Vlaams Gewest (B) & 2,5 \\
3 & Bayern (D) & 5,6 & 13 & Ostösterreich (A) & 2,1 \\
4 & Hessen (D) & 5,4 & 14 & Rhone Alpes (F) & 2,9 \\
5 & West Holland (NL) & 4,6 & 15 & Berlin (D) & 1,8 \\
6 & Schweiz & 4,1 & 16 & Lombardei (I) & 1,8 \\
7 & Eastern (UK) & 3,9 & 17 & Uusima (SF) & 1,7 \\
8 & Nordrhein-Westfalen (D) & 3,4 & 18 & Elsass (F) & 1,6 \\
9 & Kopenhagen Amt (DK) & 3,3 & 19 & Stockholm (S) & 1,6 \\
10 & Baden-Württemberg (D) & 3,0 & 20 & Madrid (E) & 1,5 \\
\hline
\end{tabular}

Quelle: Europäische Kommission (2001) 


\subsection{Lokale Nachfrage}

Die relativ gute Position Ostösterreichs ist nicht zuletzt der Anwesenheit einiger multinationaler Konzerne zu verdanken, die Forschungslabors in Wien und Umgebung betreiben. Diese Forschungsinstitute haben dabei zwei Rollen: Einerseits sind sie 'Produzenten' von industrieller Forschung, und als solche die Quelle zahlreicher lokaler Interaktionen mit Universitäten und Unternehmen, die die Quelle für Wissenstransfer sind.

Andererseits treten sie aber auch als Nachfrager von F\&E-Dienstleistungen bzw. von Wissen der Start-up-Firmen auf und können dadurch wichtige Impulse für die Dynamisierung der Biotechnologieindustrie liefern. Dabei entstehen vielfältige Kooperationen zwischen jungen Biotechnologiefirmen und traditionellen ("big pharma") Pharmakonzernen (vgl. Pyka, Saviotti 2000). Biotechnologiefirmen übernehmen einerseits die Rolle von Zulieferern ( $z$. B. von biologischen Zielstrukturen, sogenannten "Targets") und bieten andererseits Plattformtechnologien an, die als Werkzeug in den Forschungsabteilungen der Großkonzerne verwendet werden (z. B. Bioinformatik). Nur wenige - vor allem amerikanische Biotechnologiefirmen der ersten Generation - sind stärker vertikal integriert und bieten die gesamte Pharmawertschöpfungskette von Forschung über Entwicklung hin zu Zulassung und Vermarktung von Medikamenten selber an (z. B. die 1980 gegründete Amgen).

Deshalb wäre für die Bildung eines Biotechnologieclusters in Österreich eine starke lokale Nachfrage durch Pharmafirmen ein großer Vorteil. In der Schweiz ist durch die Anwesenheit der Konzernzentralen großer Pharmakonzerne wie Novartis und Roche dieser Vorteil gegeben, was mit ein Grund ist, warum es dort ca. neunmal so viele Biotechnologiefirmen wie in Österreich gibt.

Die österreichische Pharmabranche ist dagegen im internationalen Vergleich unterräpresentiert. Sie wird von den Niederlassungen internationaler Konzerne dominiert, wobei die meisten Firmen lediglich Vertriebsgesellschaften betreiben. Einige wenige Großkonzerne betreiben allerdings auch eigene Forschung in Österreich. Zu nennen sind in erster Linie Boehringer Ingelheim aus Deutschland und Novartis aus der Schweiz, die beide Labors in Wien betreiben. Boehringer Ingelheim will z.B. bis 2002 die gesamte Krebsforschung in Wien konzentrieren und betreibt außerdem das stark grundlagenforschungs-orientierte Institut für Molekulare Pathologie (IMP) in Wien ${ }^{33}$.

Der Schweizer Pharmariese Novartis hat neben seinem auf Dermatologie spezialisierten Labor in Wien mit der Tiroler Tochtergesellschaft Biochemie Kundl noch ein weiteres Standbein in Österreich. Dort ist auch das weltweite Hauptquartier der Generika-Abteilung des Konzerns angesiedelt. Biochemie Kundl hat Ende 2001 ein eigenes Antibiotika-Forschungszentrum (ABRI) mit 50 Mitarbeitern in Wien eröffnet. Insgesamt investiert Novartis nach eigenen Angaben in Österreich jährlich 90 Mio. € in F\&E und beschäftigt rund 500 ForscherInnen (1999).

33 Das IMP wurde 1985 als JV mit Genentech gegründet und hat 1988 den Betrieb aufgenommen. Mittlerweile arbeiten am IMP über 700 WissenschaftlerInnen aus mehr als 40 Nationen. 
Das amerikanische Biotechnologieunternehmen Baxter ist durch die Übernahme der Firma Immuno zu einem weiteren Akteur in Österreich geworden. Eigenen Angaben zufolge beträgt das F\&E-Budget für die 400 Mitarbeiter der Forschungslabors in Wien und Orth an der Donau 52 Mio. $€$. Weiterhin betreibt der amerikanische Konzern Eli Lilly ein klinisches Forschungszentrum in Wien, in dem 70 Mitarbeiter einen jährliches F\&E-Etat von 20 Mio. \$ zur Verfügung haben.

Die Anwesenheit dieser Forschungsinstitute ist sehr wichtig für die Entwicklung einer österreichischen Biotechnologieszene, weswegen die Politik hier die richtigen Anreize liefern muss, damit die Großkonzerne zumindest einen kleinen Teil ihrer Forschungsanstrengungen in Österreich ansiedeln.

\subsection{Finanzierung}

Wissenschaftliche Exzellenz und lokale Nachfrage sind wichtige Bausteine für eine dynamische Biotechnologieszene. Ein weiteres Element ist die Existenz von innovativen Kleinunternehmen, die bei der Umsetzung wissenschaftlicher Erkenntnisse in kommerzielle Anwendungen eine wichtige Bindegliedfunktion einnehmen. Ein großes Problem ist dabei die Finanzierung von solchen "start-ups". Kleine Unternehmen im Hochtechnologiebereich haben fast ausschließlich intangibles Kapital, weshalb die klassische Finanzierungen über Banken, die ihre Kredite durch Sachwerte absichern möchten, kaum funktioniert. Im Hochtechnologiebereich hat sich daher Venture Capital als Finanzierungsform bewährt. Investoren beteiligen sich am Eigenkapital einer Firma und partizipieren dadurch an Wachstum und Wertsteigerung des Unternehmens.

Die eingangs gemachte Festgestellung, dass Österreich bei der Anzahl der Biotechnologieunternehmen an hinterer Position in Europa rangiert, liegt nicht zuletzt an der mangelnden österreichischen Venture-Kapital-Kultur. Wie aus Tabelle 8-4 hervorgeht, ist nicht nur Österreichs Anteil von Venture Capital am BIP vergleichsweise niedrig, es geht auch nur ein relativ geringer Anteil des Venture Capitals in die Biotechnologie.

Ein gutes Signal ist allerdings, dass die Venture-Capital-Szene in Österreich in den vergangenen Jahren im europäischen Vergleich überdurchschnittlich stark gewachsen ist und sich der Abstand zu anderen europäischen Ländern daher verringert hat. So hat sich zwischen 1996 und 2000 das Volumen in Österreich von 24 Mio. $€$ auf 235 Mio. $€$ erhöht, was fast eine Verzehnfachung bedeutet. In Europa hat sich im gleichen Zeitraum das Volumen von 8 Mrd. $€$ auf $48 \mathrm{Mrd}$. $€$ lediglich versechsfacht (EVCA 2001). Ein Erfolgsbeispiel ist die Firma Intercell, die sich aus einem Institut der Universität Wien gebildet hat. Im Jahr 2001 konnten von internationalen Investoren wie Nomura und Sal. Oppenheim 27 Mio. $€$ an Venture Capital eingesammelt werden, die für die Entwicklung neuer Impfstoffe ausgegeben werden sollen. 
Tabelle 8-4: Venture Capital (1995-1999)

\begin{tabular}{lccc}
\hline Land & VC (Biotechnologie) / BIP & VC (Gesamt) / BIP & VC (Biotechnologie) / VC (Gesamt) \\
\hline Kanada & 0,032 & 0,16 & 20,3 \\
USA & 0,032 & 0,21 & 15,5 \\
OECD-19 & 0,017 & 0,14 & 12,5 \\
Belgien & 0,010 & 0,10 & 9,6 \\
Niederlande & 0,009 & 0,16 & 6,0 \\
Schweden & 0,008 & 0,08 & 10,3 \\
UK & 0,008 & 0,12 & 6,6 \\
Finnland & 0,008 & 0,07 & 10,6 \\
Schweiz & 0,007 & 0,05 & 13,6 \\
Deutschland & 0,005 & 0,06 & 9,3 \\
EU & 0,005 & 0,07 & 6,8 \\
Frankreich & 0,004 & 0,06 & 7,5 \\
Norwegen & 0,003 & 0,10 & 3,0 \\
Dänemark & 0,003 & 0,02 & 10,5 \\
Irland & 0,002 & 0,06 & 4,0 \\
Griechenland & 0,002 & 0,03 & 7,3 \\
Spanien & 0,001 & 0,05 & 2,6 \\
Österreich & 0,001 & 0,01 & 5,6 \\
Portugal & 0,000 & 0,05 & 1,0 \\
Italien & 0,000 & 0,03 & 1,3 \\
\hline
\end{tabular}

Quelle: OECD (2001)

\subsection{Forschungsförderung}

Das schwache Abschneiden Österreichs in der kommerziellen Biotechnologie hängt nicht zuletzt auch mit dem relativ späten Start dezidierter Forschungs- und Gründerprogramme auf diesem Gebiet zusammen. Dementsprechend gering war $1997 \mathrm{im}$ internationalen Vergleich der Anteil der öffentlichen Forschungsgelder im Bereich Biotechnologie an den gesamten öffentlichen Forschungsausgaben (Tabelle 8-5).

Die meisten öffentlichen Fördergelder für Biotechnologie werden über die beiden Fonds FFF $(59 \%)$ und FWF $(13,7 \%)$ ausgeschüttet (vgl. Baier, Griessler und Martinsen 2000). Die Stadt Wien rangiert mit einem Anteil von $9,3 \%$ an dritter Stelle, danach kommen die Oesterreichische Nationalbank $(5,1 \%)$ und das Land Niederösterreich $(5,1 \%)$.

In letzter Zeit sind allerdings neue Programme im Biotechnologiebereich auf Schiene gesetzt worden. Eines davon ist das Genforschungsprogramm "GenAu" des Bundesministeriums für Bildung, Wissenschaft und Kultur (BMBWK). Es sieht vor, Verbundforschungsprojekte zu fördern, die sich mit der Analyse der Struktur und Funktion des menschlichen Genoms bzw. dem wissenschaftlich interessanter Pflanzen, Tiere und Mikroorganismen beschäftigen. Das Programm hat eine Laufzeit von neun Jahren und ist mit insgesamt 100 Mio. $€$ dotiert. Es 
werden dabei überprüfbare Ziele vorgegeben: Mindestens 100 Patente und 20 bis 30 neue Firmen sollen durch das Programm generiert werden.

Ein weiteres relevantes Programm ist das von der Innovationsagentur getragene Life Science Austria, ein Programm des Bundesministeriums für Wirtschaft und Arbeit sowie des Bundesministeriums für Bildung, Wissenschaft und Kultur. Es versteht sich als zentrale Beratungs- und Koordinationsstelle für Forscher und Unternehmen. Es bietet Unterstützung in Fragen der Patentierung bzw. Patentvermarktung, der Unternehmensgründung und der Finanzierung.

"Biotechnologie Impuls Programm", in dessen Rahmen potentielle Gründer mit Beratung und finanzieller Hilfe unterstützt werden. Die Innovationsagentur war auch Projektträger eines Businessplanwettbewerbes namens "Best of Biotech".

Tabelle 8-5: Biotechnologische Forschungsausgaben in Europa (1997)

\begin{tabular}{lccc}
\hline Land & $\begin{array}{c}\text { Öffentliche F\&E-Ausgaben } \\
\text { Biotechnologie* }\end{array}$ & $\begin{array}{c}\text { Öffentliche F\&E-Ausgaben* } \\
\text { Total (GBOARD) }\end{array}$ & Anteil Biotechnologie in \% \\
\hline Deutschland & $1.048,2$ & $15.595,7$ & 6,7 \\
UK & 705,1 & $9.055,7$ & 7,8 \\
Frankreich & 560,0 & $12.683,1$ & 4,4 \\
Kanada & 261,4 & $2.581,0$ & 10,1 \\
Belgien & 181,7 & $1.314,0$ & 13,8 \\
Finnland & 94,5 & $1.165,0$ & 8,1 \\
Niederlande & 78,0 & $3.069,9$ & 2,5 \\
Schweden & 65,6 & $1.795,2$ & 3,7 \\
Dänemark & 45,2 & 945,6 & 4,8 \\
Italien & 32,1 & $7.329,6$ & 0,4 \\
Portugal & 19,2 & 781,9 & 2,5 \\
Österreich & 16,8 & $1.146,5$ & 1,5 \\
Spanien & 15,5 & $3.202,6$ & 0,5 \\
Irland & 15,0 & 229,9 & 6,5 \\
Griechenland & 6,5 & 430,9 & 1,5 \\
Island & 0,9 & 68,5 & 1,3 \\
\hline
\end{tabular}

*in Mio. \$. Wechselkurs zu Kaufkraftparitäten.

Quelle: OECD (2001), Senker (2001)

Das im Januar 2000 gestartete Kplus Zentrum für "Biomolecular Therapeutics" (BMT) in Wien ist eine weitere erwähnenswerte Initiative. Mit einem Budget von 12,35 Mio. $€$ für die ersten vier Jahre (darunter 7,4 Mio. € Fördergelder) werden 55 ForscherInnen auf dem Gebiet der Immonologie beschäftigt. Wissenschaftliche Partner sind die Universität Wien und die BOKU Wien. Auf Unternehmensseite beteiligen sich die Firmen Baxter, Biomax Informatics, Nanosearch Membrane, Novartis, Polymun Scientific und Technoclone.

Die Programme werden der Biotechnologieszene neue Impulse verleihen, wobei besonders der Standort Wien mit dem IMP in der Bohrgasse, den Forschungszentren einiger internationaler Pharmafirmen und diversen Universitätsinstituten über großes Entwicklungspotential verfügt und durchaus zu einem relevanten Cluster in Europa entwickeln könnte. 


\begin{abstract}
Biotechnologie in Wien
Der unter dem Namen "Vienna Biotech Center" firmierende Biotechnologie Cluster in Wien hat sich rund um das IMP in der Bohrgasse entwickelt. Mittlerweile arbeiten dort über 700 Wissenschaftler aus mehr als 40 Nationen. Neben dem IMP sind dort seit 1992 auch relevante Institute der Universität Wien angesiedelt (Institut für Biochemie und molekulare Zellbiologie, Institut für Mikrobiologie und Genetik, Institut für medizinische Biochemie). Im Aufbau befindet sich das Institut für molekulare Strukturbiologie und Röntgenstrukturanalyse. Unter den dort angesiedelten Unternehmen befinden sich die Firmen Intercell, Axon Neurosciences, Bender MedSystems, VBC Genomics und Veterany Animal Diognostics. In den nächsten Jahren sollen am "Vienna Biotech Center" weitere $10.000 \mathrm{~m}^{2}$ an Laborflächen errichtet werden, um den wachsenden Raumbedarf der dortigen Institute zu decken. Durch eine gemeinsame Initiative der Österreichischen Akademie der Wissenschaften (ÖAW) und der Stadt Wien wurde der Wiener Biotech-Cluster im letzten Jahr um das Institut für Molekulare Bioinformatik (IMBA) bereichert. Die Einrichtung wurde als Tochterfirma der Österreichischen Akademie der Wissenschaften gegründet und wird in Kooperation mit dem Institut für Molekulare Pathologie (IMP) betrieben. Die Stadt Wien hat die für die Ansiedlung notwendigen Flächen bereitgestellt und weitere 14 Mio. $€$ an Fördergelder vergeben. Weiterhin wird sich eine neue "non-profit" Forschungseinrichtung, die Karl Landsteiner Foundation, am VBC niederlassen.
\end{abstract}

\title{
8.6 Fazit
}

Während Österreich bei der wissenschaftlichen Basis und der Transmission in kommerzielle Anwendungen im wesentlichen im internationalen Vergleich wettbewerbsfähig ist, gibt es deutliche Schwächen bei der Dynamik der Unternehmensneugründungen. Biotechnologie, die andernorts als die Schlüsselindustrie des kommenden Jahrhunderts gesehen wird, wurde in Österreich erst relativ spät ein wichtiges Thema für die Technologiepolitik. Erst in letzter Zeit wird versucht, durch neu aufgelegte Programme (GenAu) in dieser Hinsicht Akzente zu setzen. 


\section{Literatur}

Allansdottir, A., Bonnaccorsi, A., Gambardella, A., Mariani, M., Orsenigo, L., Pammolli, F., Riccaboni, M. (2002): Innovation and Competetiveness in European Biotechnology, Brüssel: Europäische Kommission.

Baier, B., Griessler, E., Martinsen, R. (2000): European Biotechnology Innovation System: National Case Study of Austria, Wien: IHS.

Basberg, B.L. (1987): Patents and the Measurement of Technological Change: A survey of the literature, Research Policy, vol. 16, pp. 131-141.

Bellak, C. (2002): Große Multis in kleinen Ländern - Fallstudien. in: Bundesministerium für Wirtschaft und Arbeit (Hrsg.): Österreichs Außenwirtschaft. Wien

Bertschek, I., Fryges, H. (2002): the Adoption of Business-to-Business E-Commerce: Empirical Evidence for German Companies. ZEW Discussion Paper No. 02-05. Mannheim.

Boston Consulting Group (2000): Rennsaison: B2B-E-Commerce in Deutschland. München.

Boston Consulting Group (2001): Biotechnologie in Österreich, Düsseldorf.

Bundesministerium für Finanzen (2002A), Konjunkturbelebungsgesetz 2002 - Gesetzestext, http://www.bmf.gv.at/steuern/neuegesetze/konjunkturrv/gesetzestext.pdf.

Bundesministerium für Finanzen (2002B), Konjunkturbelebungsgesetz 2002 - Erläuterungen, http://www.bmf.gv.at/steuern/neuegesetze/konjunkturrv/erlaeuterungen.pdf.

Callon, M. (1994): Is Science a Public Good? Science, Technology and Human Values, 19(4), 395-424.

Cohen, W. (1995): Empirical Studies of Innovative Activity, in Stoneman (ed): handbook of the Economics of Innovation and Technological Change, S. $182-264$.

Dachs, B., Knoll, N. (2001): Screening eEurope. Technologiepolitik für die Informationsgesellschaft. Studien im Rahmen des TIP, Wien.

Dachs, B., Wagner, P. (2001): IT Outlook 2002. Recent Developments in Austrian IT Policies. Report on behalf of the Federal Ministry of Transport, Innovation and Technology and the Federal Chancellery. OEFZS-S-0141

EITO: European Information Technology Observatory 2002. Frankfurt am Main.

Ernst \& Young (2001): Integration. Ernst \& Young's Eight Annual European Life Science Report 2000, London.

EUREKA (2001): Annual Report 2000/2001. http://www.eureka.be/ifs/files/ifs/jspbin/eureka/ifs/jsps/publicHome.jsp.

Europäische Kommission (1997): Erster Aktionsplan für Innovation in Europa, EUR-OP, Luxemburg

Europäische Kommission (1999): European Technology Assessment Network: International Compendium of Indirect Schemes \& Measures for Supporting RTD in Enterprises, Brüssel, 1999.

Europäische Kommission (1999A): European Technology Assessment Network: A Report on the Promotion in Research and Innovation Through Indirect Measures, Brüssel, 1999.

Europäische Kommission (2001): European Competitiveness Report. Brüssel.

Europäische Kommission (2001a): Galileo - Unverzichtbar für Europa. http://www.galileoaustria.at/pdf/DE.pdf

European Commission (2002): Corporation Tax and Innovation, DG Enterprise. Innovation Directorate, Innovation Papers, 2002, (19). 
Europäischer Rat (2002): Schlussfolgerungen des Vorsitzes, Europäischer Rat Barcelona 15. und 16. März 2002. http://ue.eu.int/de/Info/eurocouncil/index.htm

European Space Agency (2001): Annual Report 2000. http://esapub.esrin.esa.it/annuals/annuals.htm

European Space Agency (2002): ESA Facts and Figures. http://www.esa.int/export/esaCP/GGG4SXG3AEC index $0 . \mathrm{html}$

EVCA (2001): Annual Survey of Pan-European Private Equity and Venture Capital Activity, Zavantem.

Guellec, D., van Pottelsberghe, B. (2000): The Impact of Public R\&D Expenditure on Business R\&D, STI Working Papers, 2000, (4).

Hall, B., van Reenen, J. (2000): How Effective are Fiscal Incentives for R\&D? A Review of the Evidence, Research Policy, 29(3-5), S. 449-469.

Hutschenreiter, G. (2002): Steuerliche Förderung von Forschung und Entwicklung. WIFO-Monatsberichte 2002, 75(2), S. 121-131.

Hutschenreiter, G., Peneder, M.(1997): Österreichs Technologielücke im Außenhandel. WIFOMonatsberichte, 1997, 70 (2), S. 103-114.

Hutschenreiter, G., Aiginger, K. (2001): Steuerliche Anreize für Forschung und Entwicklung. Internationaler Vergleich und Reformvorschläge für Österreich, Studie des WIFO im Auftrag des Rates für Forschung und Technologieentwicklung, Wien. http://titan.wsr.ac.at:8880/wifosite/wifosite.get abstract type?p language=1\&pubid=20540.

Informationssekretariat Biotechnologie (2001): Zahlen zur roten Biotechnologie, http://www.i-s-b.org.

Jaffe, A.B., Trajtenberg, M., Henderson, R. (1993): Geographic Localization of Knowledge Spillovers as Evidenced by Patent Citations. Quarterly Journal of Economics 108, pp. 577-598.

Joanneum Research (2002): Erhebung der business-to-business-Aktivitäten österreichischer Unternehmen. Unveröffentlicht, InTeReg, Wien.

Knoll, N. (2001): Anwendung der Lasertechnik in der österreichischen Industrie. Ökonomische Potentiale des Lasertechnikeinsatzes. Studie im Auftrag des BMVIT. WIFO, Wien.

Lehrl, S. (1993): Die Forschungsqualität deutscher Mediziner: Normen und Bewertungen, Nürnberg.

Leo, H., Schwarz, G., Geider, M., Pohn-Weidinger, S., Polt, W. (2002): Die direkte Technologieförderung des Bundes, Studie im Auftrag des Bundesministeriums für Wirtschaft und Arbeit, Joanneum Research und WIFO, Graz - Wien

Narin, F. und Olivastro, D. (1988): Technology Indicators Based on Patents and Patent Citation. in Raan, van, A.F.J.(ed.) Handbook of Quantitative Studies of Science and Technology, pp. 465-507, Amsterdam: North Holland.

OECD (1994): Frascati Manual 1993. Proposed Standard Practice for Surveys of Research and Experimental Development, Paris, 1994. http://www.oecd.org/pdf/M00003000/M00003664.pdf

OECD (1998): Technology, Productivity and Job Creation. Best Policy Practices. Paris.

OECD (2000): Science, Technology and Industry Outlook 2000 Paris.

OECD (2001): OECD Science, Technology and Industry Scoreboard. Towards a Knowledge-based Economy, Paris. http://www1.oecd.org/publications/e-book/92-2001-03-1-2987/

OECD (2001a): The New Economy Beyond the Hype. OECD Growth Project Final Report. Paris. http://www.oecd.org/pdf/M00018000/M00018622.pdf

OECD (2001b): The Internet and Business Performance. Paris. http://www.oecd.org/pdf/M00023000/M00023758.pdf

OECD (2001c): Education at a Glance 2001. Paris. 
OECD (2001d): Public Funding of R\&D: Emerging Policy Issues, DSTI/STP(2001)2. Paris.

Österreichische Bundesregierung, Konjunkturgipfel der Bundesregierung, 5. Dezember 2001, http://www.bmf.gv.at/presse.

Pavitt, K. (1984): Sectoral Patterns of Technical Change: Towards a Taxonomy and a Theory, in: Research Policy 13 (1984), S. 343 - 373.

Pavitt, K. (1988): Uses and Abuses of Patent Statistics, in Raan, van, A.F.J.(ed.) Handbook of Quantitative Studies of Science and Technology, pp. 509-535. Amsterdam: North Holland.

Peneder, M. (1999): Intangible Investment and Human Resources. The New WIFO Taxonomy of Manufacturing Industries. WIFO Working Paper 114/1999, http://titan.wsr.ac.at/wifosite/wifosite.get abstract type?p language=1\&pubid=1585

Peneder, M. (2001): Eine Neubetrachtung des „Österreich-Paradoxon“, in WIFO-Monatsberichte 12/2001, S. 737-748.

Peneder, M., Aiginger, K., Hutschenreiter, G., Marterbauer, M. (2001): Structural Change and Economic Growth, study commissioned by the Federal Ministry for Economic Affairs and Labour, WIFO, Vienna

Peneder, M. Kaniovski, S., Dachs, B. (2001): External Services, Structural Change and Industrial Performance. DG Enterprise Working Paper No 3, http://europa.eu.int/comm/enterprise/library/enterprise-papers/paper3.htm

Pyka, A., Saviotti, P. P. (2000): Innovation Networks in Biotechnology. SEIN Project Report, Part III, http://www.uni-bielefeld.de/iwt/sein/biorepor.pdf

Salter, A. J., Martin, B. R. (2001): The Economic Benefits of Publicly Funded Basis Research: A Critical Review, Research Policy 30, pp. 509-532.

Schibany, A., Jörg, L., Gassler, H., Warta, K., Sturn, D., Polt, W., Streicher, G., Luukkonen, T., Arnold, E. (2001): Evaluierung der österreichischen Beteiligung am 4. Rahmenprogramm der EU für Forschung, technologische Entwicklung und Demonstration, Wien.

Senker, J. (2001): European Biotechnology Innovation System: Analysis of the Biopharmaceutical Sector, Brüssel: Europäische Kommission.

Shapiro, C., Varian, H. (1999): Information Rules. A Strategic Guide to the Network Economy. Harvard Business School Press, Boston.

Statistik Austria (2001): E-Commerce 2000/2001. Ergebnisse der Ersten Europäischen Piloterhebung. Wien.

Statistik Austria (2002): Statistisches Jahrbuch Österreichs 2002, Kapitel 5 Wissenschaft und Technologie. Wien

Stephan, P. (1996): The Economics of Science, Journal of Economic Literature, Vol. XXXIV (September 1996), pp 1199-1235.

Topolnik, M., Dinhobl, G., Wiesmüller, M., Unger, M., Hübner, M., Hartl, M., Gottmann, E. (2001): PROVISO-Managementinformation. 5. EU-Rahmenprogramm. Die österreichische Beteiligung im europäischen Kontext (Stand Oktober 2001); beschränkte Verbreitung; Wien, bmvit und bmbwk 2001.

Van den Besselaar, P. Heimeriks, G. (2000) Codification and Self-Organization of the European ScienceTechnology-Innovation System. The Self-Organization of the European Information Society. TSER. Final report of task 4, May 2000.

Warda, J. (2002): Measuring the Value of R\&D Tax Treatment in OECD Countries, in STI Review, 2002, (27), 185-211. 


\section{STATISTISCHER ANHANG}

(erstellt von Statistik Austria)

\subsection{F\&E-Ausgaben des Bundes 2002 (Tabellen 1 bis 8)}

1.2.1. Die Ausgaben des Bundes für in Österreich durchgeführte $F \& E$ werden 2002 rund 1457 Mio. € (20,0 Milliarden Schilling) erreichen, sie liegen damit um rund 7,3 Prozent über dem Vorjahresniveau, um 32,7 Prozent über dem Niveau von 1998, um 52,2 Prozent über dem Niveau von 1993 und um 135,8 Prozent über dem Niveau von 1989 (siehe Tabelle 1). Gemäß der zugrundeliegenden Methodik handelt es sich bei den in Tabelle 1 ausgewiesenen Ausgaben des Bundes für in Österreich durchgeführte F\&E für die Jahre 2001 und 2002 um Bundesvoranschlagswerte.

Im Bundesvoranschlag 2001 sind im VA-Ansatz 1/5182 unter dem Titel „Offensivprogramm F\&E“ insgesamt 508,7 Mio. € (7 Milliarden Schilling) veranschlagt. Die volle Berücksichtigung dieser Förderungsmittel im Jahre 2001 würde die Schätzung der Finanzierungsanteile des Bundes sowohl für die Jahre 2001 und 2002 als auch für die Folgejahre stark verzerren. Deshalb sind - abweichend von der Beilage T - in die Schätzung des Finanzierungsanteiles des Bundes durch Statistik Austria nur jene Mittel eingegangen, welche im Jahre 2001 tatsächlich ausgegeben wurden bzw. welche 2002 der Forschung zufließen sollen. So wurden entsprechend den bisher vorliegenden Informationen über erfolgte Auszahlungen im Jahr 2001 130,2 Mio. € (1,791 Mrd. Schilling) berücksichtigt. Für das Jahr 2002 wird seitens Statistik Austria von der Annahme ausgegangen, dass ca. 218 Mio. $€$ (3 Mrd. ATS) ausgegeben werden, die Inanspruchnahme der restlichen Mittel (ca. 160 Mio. € bzw. 2,2 Mrd. ATS) ist für die F\&E-Ausgaben 2003 vorgesehen.

1.2.2. Zusätzlich wird der Bund im Jahre 2002 Beitragszahlungen an internationale Organisationen, die Forschung und Forschungsförderung als Ziel haben, in Höhe von 44,307 Mio. € leisten (Beilage T des Amtsbehelfes zum Bundesfinanzgesetz 2002/Teil a).

1.2.3. Die Ausgaben des Bundes für Forschung und Forschungsförderung - also einschließlich der forschungswirksamen Anteile an den Beitragszahlungen an internationale Organisationen - sind in der Beilage T des Amtsbehelfes zum Bundesfinanzgesetz, Teil a und Teil b, zusammengefasst dargestellt (siehe Tabelle $3 \mathrm{im}$ Tabellenteil). Die Werte aus dem Bundesrechnungsabschluss 2000 enthalten auch die im Jahre 2000 eingesetzten forschungswirksamen Gelder aus den „Technologie- und Exportoffensiven 1998 und 1999“ (26,762 Mio. €).

Wie aus Tabelle $2 \mathrm{im}$ Tabellenteil ersichtlich, werden die Ausgaben des Bundes für Forschung und Forschungsförderung 2002 rund 1,3 Milliarden $€$ erreichen.

1.2.3.1. Von diesen Ausgaben des Bundes für Forschung und Forschungsförderung entfallen im Jahre 2002 (siehe Tabelle 2)

76,0 Prozent auf das BMBWK (einschließlich anteiliger Bauausgaben)

14,7 Prozent auf das BMVIT

3,9 Prozent auf das BMF (einschließlich der im Budgetkapitel 54 veranschlagten Mittel

für Mietenzahlungen an die BIG in der Höhe von 17,308 Mio €)

3,4 Prozent auf das BMLFUW (einschließlich anteiliger Bauausgaben)

0,8 Prozent auf das BMSG

0,6 Prozent auf das BMWA

0,4 Prozent auf das BKA

0,2 Prozent auf das BMAA 
1.2.3.2. Für die Bundesrechnungsabschlussdaten 2000 und die Voranschlagsdaten für die Jahre 2001 und 2002 liegen auch die funktionelle Aufgliederung der Ausgaben des Bundes für Forschung und Forschungsförderung (einschließlich der forschungswirksamen Anteile an den Beitragszahlungen an internationale Organisationen) nach sozio-ökonomischen Zielsetzungen vor (siehe Tabellen 4 - 8).

In der Auswertung der Voranschlagsdaten 2001 nach sozio-ökonomischen Zielsetzungen wurden von den Mitteln der „Forschungs- und Technologieoffensive“, welche im Jahre 2001 im Budgetkapitel 51 rücklagefähig mit insgesamt 508,710 Mio. € (7 Mrd. Schilling) veranschlagt waren („Offensivprogramm F\&E“; siehe Tabelle 3) nur jener Anteil in der Höhe von 130,2 Mio. $€(1,791$ Mrd. Schilling) berücksichtigt, welcher nach den derzeit vorliegenden Informationen im Jahre 2001 tatsächlich zur Auszahlung gelangte.

2002 kommen folgenden sozio-ökonomischen Zielsetzungen die stärksten Anteile an den Gesamtausgaben des Bundes für Forschung und Forschungsförderung zu (siehe Tabelle 8):

Förderung der allgemeinen Erweiterung des Wissens: 31,5 Prozent

Förderung des Gesundheitswesens: 23,0 Prozent

Förderung von Handel, Gewerbe und Industrie: 15,2 Prozent

Förderung der sozialen und sozio-ökonomischen Entwicklung: 7,0 Prozent

Förderung der Erforschung der Erde, der Meere, der Atmosphäre und des Weltraumes: 6,6 Prozent

Förderung der Land- und Forstwirtschaft: 6,0 Prozent

Förderung des Transport-, Verkehrs- und Nachrichtenwesens: 2,7 Prozent:

\subsection{F\&E-Ausgaben der Bundesländer 2002}

Die F\&E-Ausgaben der Bundesländer unter Einschluss der F\&E-Ausgaben der Landeskrankenanstalten werden von Statistik Austria - unter Heranziehung der Meldungen der Ämter der Landesregierungen - für 2002 mit 267,8 Mio. € (3,685 Mrd. Schilling) geschätzt. Sie liegen damit um rund 3 Prozent über dem Niveau von 2001, um 88,1 Prozent über dem Ergebnis der F\&E-Erhebung 1998 und um 106,5 Prozent über dem Erhebungsergebnis von 1993 (siehe Tabelle 1).

\section{Auswertungen der Faktendokumentation 2000 (Tabellen 9 bis 14)}

Für das Jahr 2000 liegen folgende Auswertungen vor:

a) nach Förderungsempfängern bzw. Auftragnehmern,

b) nach sozio-ökonomischen Zielsetzungen sowie

c) nach Wissenschaftszweigen.

Wie für die vergangenen Jahre wurden auch für 2000 jeweils Auswertungen unter Einschluss der "großen" Globalförderungen (d.h. der Globalförderungen an den Fonds zur Förderung der wissenschaftlichen Forschung, den Forschungsförderungsfonds für die gewerbliche Wirtschaft, die Ludwig Boltzmann-Gesellschaft, die Österreichische Akademie der Wissenschaften und das Österreichische Forschungszentrum Seibersdorf) und solche ohne dieselben erstellt.

Die in der Faktendokumentation 2000 nicht vollständig ausgewiesenen Förderungsmittel seitens des BMBWK an den FWF sowie seitens des BMVIT an den FFF wurden auf der 
Basis des Bundesrechnungsabschlusses 2000 ermittelt und in die entsprechenden Auswertungen einbezogen.

Unter Berücksichtigung dieser Mittel entfielen 76,5 Prozent der Summe der ausbezahlten Beträge auf die erwähnten "großen" Globalförderungen.

Die Tabellen sind jeweils nach vergebenden Ressorts gegliedert. Von den gesamten erfassten Mitteln (d.h. unter Einschluss der "großen" Globalförderungen) wurden 61,7 Prozent vom BMVIT, 35,7 Prozent vom BMBWK sowie der Rest (2,6 Prozent) von den anderen Ressorts ausbezahlt.

Werden die "großen" Globalförderungen aus der Betrachtung ausgeklammert, ergibt sich, dass 49,2 Prozent der Mittel vom BMBWK, 39,6 Prozent vom BMVIT, 7,5 Prozent vom BMLFUW, 1,4 Prozent vom BMSG, 1,4 Prozent vom BMWA sowie der Rest (0,9 Prozent) von den anderen Ressorts vergeben wurden.

Die Auswertung nach Förderungsempfängern bzw. Auftragnehmern unter Einschluss der "großen" Globalförderungen (siehe Tabelle 9) ergab, dass - abgesehen von den über die beiden Forschungsförderungsfonds vergebenen Förderungen (FFF: 22,4 Prozent; FWF: 21,5 Prozent) 19,4 Prozent der Teilbeträge 2000 an Förderungsempfänger bzw. Auftragnehmer im Hochschulsektor, 17,8 Prozent an solche im Unternehmenssektor, und 15,8 Prozent an Einrichtungen im Sektor Staat vergeben wurden. Unter Einbeziehung der über den FFF vergebenen Förderungen gingen demnach 40,2 Prozent der im Jahre 2000 ausbezahlten Beträge an den Unternehmenssektor.

Lässt man die "großen" Globalförderungen außer Betracht (siehe Tabelle 10), so wurden 37,8 Prozent der Förderungsbeträge 2000 an Förderungsempfänger im Sektor Staat, 25,4 Prozent an solche im Hochschulsektor, 20,5 Prozent an Auftragnehmer im Unternehmenssektor und 12,1 Prozent an den privaten gemeinnützigen Sektor vergeben.

Bei der Auswertung nach sozio-ökonomischen Zielsetzungen unter Einschluss der "großen" Globalförderungen (siehe Tabelle 11) ergab sich, dass 2000 der "Förderung der allgemeinen Erweiterung des Wissens" mit 31,8 Prozent das größte Gewicht zukam. 27,6 Prozent der Förderungsmittel waren der Kategorie "Förderung von Handel, Gewerbe und Industrie" zurechenbar. Werden die "großen" Globalförderungen in die Berechnungen nicht einbezogen (siehe Tabelle 12), so entfallen auf die "Förderung der allgemeinen Erweiterung des Wissens" 37,9 Prozent der gesamten Förderungsmittel, auf die "Förderung der sozialen und sozio-ökonomischen Entwicklung" 18,0 Prozent und auf die "Förderung von Handel, Gewerbe und Industrie" sowie die "Förderung des Umweltschutzes" 9,1 Prozent.

Die Auswertung nach Wissenschaftszweigen unter Einschluss der "großen" Globalförderungen (siehe Tabelle 13) ergab, dass 2000 die größten Anteile an den Förderungsbeträgen auf die Technischen Wissenschaften (36,6 Prozent) und die Naturwissenschaften (32,0 Prozent) entfielen. Unter Ausklammerung der "großen" Globalförderungen (siehe Tabelle 14) entfielen auf die Sozialwissenschaften 27,5 Prozent, auf die Technischen Wissenschaften 26,5 Prozent und auf die Naturwissenschaften 21,6 Prozent der im Jahre 2000 ausbezahlten Förderungsbeträge.

\section{Universitätsjahresrechnung 2002 (Tabelle 15)}

\section{Bundespersonal an Instituten und Kliniken nach Wissenschaftszweigen und Beschäftigtenkategorien (Tabelle 15)}

Von Statistik Austria (Bundesanstalt Statistik Österreich) wurden für den universitären Bereich auf der Grundlage des nach Instituten (bzw. Kliniken) untergliederten Stellenplans für das wissenschaftliche und nichtwissenschaftliche Personal zum Stand Jänner 2002 unter Heranziehung der aus der F\&E-Erhebung 1998 stammenden Koeffizienten 
(Arbeitszeitverteilung) Vollzeitäquivalentwerte für F\&E, für Lehre und Ausbildung sowie für sonstige Tätigkeiten nach Wissenschaftszweigen (für das Bundespersonal) ermittelt.

Planstellen an den Universitäten der Künste sind in dieser Auswertung nicht berücksichtigt. Demnach waren zum Stichtag 1. 1. 2002 an Österreichs Universitäten (ohne Universitäten der Künste) insgesamt 6 805,1 Vollzeitäquivalente für F\&E eingesetzt, welche sich wie folgt auf die Wissenschaftszweige verteilten:

Wissenschaftszweige

Naturwissenschaften

Technische Wissenschaften

Humanmedizin (einschl. Kliniken)

Land- und Forstwirtschaft,

Veterinärmedizin

Sozialwissenschaften

Geisteswissenschaften

INSGESAMT
VZÄ für F\&E

1743,2

945,6

1920,7

322,1

1134,0

739,5

6805,1 in Prozent

25,6

13,9

28,2

4,7

16,7

10,9

100,0

Es sei darauf hingewiesen, dass die zugrundeliegenden Planstellenwerte, die natürlich auch unbesetzte Planstellen einschließen, das Instituts- bzw. Klinikpersonal umfassen, während das Personal der zentralen Universitätsverwaltungen ausgeschlossen ist und auch refundiertes Personal bzw. an den Instituten (Kliniken) tätiges Nicht-Bundespersonal gleichfalls nicht einbezogen ist.

\section{Erhebungen von Statistik Austria über F\&E 1998 (Tabellen 16 bis 33):}

Statistik Austria (die Bundesanstalt Statistik Österreich) hat über das Berichtsjahr 1998 statistische Erhebungen über Forschung und experimentelle Entwicklung bei den F\&E betreibenden Institutionen durchgeführt. Von diesen auf der Basis der internationalen Standards und Richtlinien durchgeführten, sehr detaillierten (Voll-)Erhebungen wurden folgende Erhebungsbereiche erfasst: Universitätsinstitute, Universitätskliniken, F\&E betreibende Einrichtungen der Universitäten der Künste, Österreichische Akademie der Wissenschaften, Versuchsanstalten an den HTL, Sektor Staat (dieser umfasst: sonstige Forschungseinrichtungen des Bundes, Forschungseinrichtungen der Länder, der Gemeinden, der Kammern, der Sozialversicherungsträger, sowie - seit der Revision 1993 des Frascati-Manuals - auch Institute und Forschungsstellen der Ludwig BoltzmannGesellschaft und private Institutionen, welche überwiegend öffentlich finanziert und/oder kontrolliert werden), privater gemeinnütziger Bereich und Unternehmenssektor (dieser umfasst neben dem großen Bereich der firmeneigenen Forschung auch den kooperativen Bereich, welcher das Österreichische Forschungszentrum Seibersdorf einschließt, sowie die Bereiche der Ziviltechniker(büros) und Kraftwerksgesellschaften).

Zum Verständnis der folgenden Darstellung sei noch eine Erläuterung zur Klassifizierung der forschungsstatistischen Daten vorausgeschickt. Nach den Frascati-Handbuch-Richtlinien werden vier Durchführungssektoren ("sectors of performance") unterschieden, d. s. weitgehend auf der Basis des Systems Volkswirtschaftlicher Gesamtrechnungen der Vereinten Nationen (SNA) definierte volkswirtschaftliche Sektoren, welchen die F\&E durchführenden Institutionen angehören: Hochschulsektor, Sektor Staat, privater gemeinnütziger Sektor, Unternehmenssektor.

Diese setzen sich in Österreich wie folgt zusammen:

- Der Hochschulsektor umfasst die Universitäten (Institute und Kliniken), die Universitäten der Künste, die Österreichische Akademie der Wissenschaften und die Versuchsanstalten an den Höheren Technischen Bundeslehranstalten. 
- Der Sektor Staat umfasst Bundesinstitutionen (unter Ausklammerung der im Hochschulsektor zusammengefassten -), Landes-, Gemeinde-, Kammer-Institutionen sowie Einrichtungen von Sozialversicherungsträgern; die Landeskrankenanstalten zählen ebenfalls zu diesem Sektor. Seit der Revision 1993 des Frascati-Manuals werden SNA-konform dem Sektor Staat auch die Institute und Forschungsstellen der Ludwig Boltzmann-Gesellschaft sowie private Institutionen, welche vorwiegend öffentlich finanziert und/oder kontrolliert sind, zugerechnet.

- Der private gemeinnützige Sektor umfasst alle privaten gemeinnützigen Institutionen ohne Erwerbscharakter (d. s. alle jene F\&E-Einrichtungen, deren Status ein vorwiegend privater oder privatrechtlicher, konfessioneller oder sonstiger nicht öffentlicher ist), ausgenommen jene, welche vornehmlich Dienstleistungen für Unternehmen erbringen (und daher jenem Bereich zugeordnet werden).

- Der Unternehmenssektor umfasst den kooperativen Bereich und die Bereiche der Ziviltechniker und der Kraftwerksgesellschaften sowie den großen Bereich der firmeneigenen Forschung (Firmeneigener Bereich). Im kooperativen Bereich sind vornehmlich kooperative Forschungsinstitute (Branchenforschungsinstitute) sowie die Forschungsgesellschaft Joanneum, die AVL List GmbH und das Österreichische Forschungszentrum Seibersdorf zusammengefasst. Im Bereich der firmeneigenen Forschung sind private und öffentliche Industrieunternehmen zusammengefasst.

Die forschungsstatistischen Daten der von Statistik Austria erhobenen Bereiche werden gemäß internationaler und supranationaler Empfehlungen (Frascati-Handbuch) standardmäßig nach folgenden Klassifikationen zugeordnet bzw. aggregiert:

- Wissenschaftszweige (anwendbar auf alle Erhebungsbereiche mit Ausnahme des Subsektors „Firmeneigene Forschung“ des Unternehmenssektors) siehe hiezu auch die Tabellen 16 bis $27 \mathrm{im}$ Tabellenteil

- sozio-ökonomische Zielsetzungen (anwendbar auf alle Erhebungsbereiche mit Ausnahme des Subsektors „Firmeneigene Forschung“ des Unternehmenssektors) siehe hiezu auch die Tabellen 31 bis 33 im Tabellenteil

- Wirtschaftszweige (ÖNACE bzw. NACE Rev.1), anwendbar nur auf die Daten aus dem Unternehmenssektor (alle Subsektoren)

siehe hiezu auch die Tabelle $27 \mathrm{im}$ Tabellenteil des Forschungs- und Technologieberichts 2001

Die Hauptergebnisse der Erhebungen 1998 betreffend die Beschäftigten sind bereits im Forschungs- und Technologiebericht 2001 enthalten.

In Ergänzung hiezu werden nunmehr die endgültigen Ergebnisse betreffend die Ausgaben für F\&E sowie deren Aufgliederung nach der Herkunft der Mittel nachgereicht (siehe Tabellen 16 bis 30 ).

\footnotetext{
Ausgaben für F\&E (Tabelle 28)

Die Zusammenführung der Ergebnisse der Erhebungen von Statistik Austria im öffentlichen Bereich (unter Einbeziehung bzw. Hinzurechnung der Landeskrankenanstalten-Schätzung: 1025 Mio. ATS) mit jener im privaten Bereich zeigt, dass 1998 in sämtlichen Durchführungssektoren 46,783 Mrd. ATS für F\&E ausgegeben wurden, davon 29,7\% im Hochschulsektor, 6,4\% im Sektor Staat, 0,3\% im privaten gemeinnützigen Sektor, 5,5\% im kooperativen Bereich und im Bereich der Ziviltechniker sowie 58,0\% im Bereich der firmeneigenen Forschung (unter Einschluss der Kraftwerksgesellschaften).
} 


\section{Finanzierung der Ausgaben für F\&E}

Von der Gesamtsumme der Bruttoinlandsausgaben für F\&E wurden 1998 41,7\% vom Unternehmenssektor, 37,8\% vom öffentlichen Sektor, 20,1\% vom Ausland (unter Einschluss der EU) und 0,4\% vom privaten gemeinnützigen Sektor finanziert (Tabelle 29).

\section{F\&E-Ausgaben nach Bundesländern}

Tabelle $30 \mathrm{im}$ Tabellenteil schließt die F\&E-Ausgaben nach Bundesländern und Ausgabenarten auf. Demnach entfallen auf Wien 48,5 Prozent, auf die Steiermark 17,6 Prozent, auf Oberösterreich 11,6 Prozent, auf Tirol 7,6 Prozent, gefolgt von den Bundesländern, welche mit Ausnahme von Kärnten und Salzburg keine Universitäten beheimaten (Niederösterreich 5,8 Prozent, Kärnten 3,6 Prozent, Salzburg 2,7 Prozent, Vorarlberg 2,2 Prozent und Burgenland 0,2 Prozent).

Sozio-ökonomische Zielsetzungen (Tabellen 31 bis 33)

Die Gliederung der forschungsstatistischen Daten aus allen Erhebungsbereichen mit Ausnahme des Bereichs der firmeneigenen Forschung erfolgt auch nach einer zweiten Standardklassifikation, den sozio-ökonomischen Zielsetzungen (zu deren Verwirklichung die F\&E-Aktivitäten dienen). Die von der Statistik Austria verwendete ÖSTAT-Systematik (13 Kategorien) wurde schon seinerzeit so konzipiert, dass die nach ihr gegliederten österreichischen Daten durch Umsteigeschlüssel auch entsprechend den von der OECD und der UNESCO eingesetzten Systematiken der sozio-ökonomischen Zielsetzungen klassifiziert werden können.

\section{F\&E-Ausgaben 1999 im internationalen Vergleich (Tabelle 34)}

Die Übersichtstabelle (siehe Tabelle 34 im Tabellenteil) zeigt anhand der wichtigsten F\&Erelevanten Kennzahlen die Position Österreichs im Vergleich zu den anderen Mitgliedstaaten der Europäischen Union bzw. der OECD. 
TABELLENÜBERSICHT

\begin{tabular}{|c|c|}
\hline Tabelle & Bezeichnung \\
\hline 1 & $\begin{array}{l}\text { Globalschätzung 2002: Bruttoinlandsausgaben für F\&E. } \\
\text { Finanzierung der in Österreich durchgeführten Forschung und experimentellen Entwicklung } 1981-2002 \text { in } \\
\text { ATS und in EURO }\end{array}$ \\
\hline $1 \mathrm{a}$ & $\begin{array}{l}\text { Globalschätzung 2002: Bruttoinlandsausgaben für F\&E. } \\
\text { Finanzierung der in Österreich durchgeführten Forschung und experimentellen Entwicklung } 1981 \text { - } 2002 \text { in } \\
\text { Prozent des BIP }\end{array}$ \\
\hline 2 & $\begin{array}{l}\text { Ausgaben des Bundes für Forschung und Forschungsförderung } 1999 \text { bis } 2002 \text { nach Ressorts. Aufgliederung } \\
\text { der Beilage T der Amtsbehelfe zu den Bundesfinanzgesetzen } 2001 \text { und } 2002 \text { (Teil a und Teil b) }\end{array}$ \\
\hline 3 & $\begin{array}{l}\text { Forschungswirksame Ausgaben des Bundes } 2000 \text { bis } 2002 \text { nach Ressorts. Beilage T des Amtsbehelfes zum } \\
\text { Bundesfinanzgesetz } 2002 \text { (Teil a und b) }\end{array}$ \\
\hline 4 & $\begin{array}{l}\text { Ausgaben des Bundes } 1993 \text { bis } 2002 \text { für Forschung und Forschungsförderung nach sozio-ökonomischen } \\
\text { Zielsetzungen (ÖSTAT-Systematik) und Berichtsjahren (in EURO) }\end{array}$ \\
\hline 5 & $\begin{array}{l}\text { Ausgaben des Bundes } 1993 \text { bis } 2002 \text { für Forschung und Forschungsförderung nach sozio-ökonomischen } \\
\text { Zielsetzungen (ÖSTAT-Systematik) und Berichtsjahren (in ATS) }\end{array}$ \\
\hline 6 & $\begin{array}{l}\text { Ausgaben des Bundes } 2000 \text { für Forschung und Forschungsförderung nach sozio-ökonomischen } \\
\text { Zielsetzungen (ÖSTAT-Systematik) und Ressorts (in EURO) }\end{array}$ \\
\hline 7 & $\begin{array}{l}\text { Ausgaben des Bundes } 2001 \text { für Forschung und Forschungsförderung nach sozio-ökonomischen } \\
\text { Zielsetzungen (ÖSTAT-Systematik) und Ressorts (in EURO) }\end{array}$ \\
\hline 8 & $\begin{array}{l}\text { Ausgaben des Bundes } 2002 \text { für Forschung und Forschungsförderung nach sozio-ökonomischen } \\
\text { Zielsetzungen (ÖSTAT-Systematik) und Ressorts (in EURO) }\end{array}$ \\
\hline 9 & $\begin{array}{l}\text { Forschungsförderungen und Forschungsaufträge des Bundes } 2000 \text { (einschließlich "große" Globalförderungen) } \\
\text { nach Förderungsempfängern bzw. Auftragnehmern (gegliedert nach volkswirtschaftlichen Sektoren/Bereichen) } \\
\text { und vergebenden Ressorts }\end{array}$ \\
\hline 10 & $\begin{array}{l}\text { Forschungsförderungen und Forschungsaufträge des Bundes } 2000 \text { (ohne "große" Globalförderungen) nach } \\
\text { Förderungsempfängern bzw. Auftragnehmern (gegliedert nach volkswirtschaftlichen Sektoren/Bereichen) und } \\
\text { vergebenden Ressorts }\end{array}$ \\
\hline 11 & $\begin{array}{l}\text { Forschungsförderungen und Forschungsaufträge des Bundes } 2000 \text { (einschließlich "große" Globalförderungen) } \\
\text { nach sozio-ökonomischen Zielsetzungen und vergebenden Ressorts (in ATS) }\end{array}$ \\
\hline 12 & $\begin{array}{l}\text { Forschungsförderungen und Forschungsaufträge des Bundes } 2000 \text { (ohne "große" Globalförderungen) nach } \\
\text { sozio-ökonomischen Zielsetzungen und vergebenden Ressorts (in ATS) }\end{array}$ \\
\hline 13 & $\begin{array}{l}\text { Forschungsförderungen und Forschungsaufträge des Bundes } 2000 \text { (einschließlich "große" Globalförderungen) } \\
\text { nach Wissenschaftszweigen und vergebenden Ressorts (in ATS) }\end{array}$ \\
\hline 14 & $\begin{array}{l}\text { Forschungsförderungen und Forschungsaufträge des Bundes } 2000 \text { (ohne "große" Globalförderungen) nach } \\
\text { Wissenschaftszweigen und vergebenden Ressorts (in ATS) }\end{array}$ \\
\hline 15 & $\begin{array}{l}\text { Universitäts-Jahresrechnung 2002: Bundespersonal an Instituten und Kliniken gegliedert nach } \\
\text { Wissenschaftszweigen und Beschäftigtenkategorien (VZÄ-Schätzung auf der Basis des Stellenplanes 1. 1. } \\
\text { 2002) }\end{array}$ \\
\hline
\end{tabular}




\begin{tabular}{|c|c|}
\hline 16 & $\begin{array}{l}\text { Hochschulsektor: Ausgaben für Forschung und experimentelle Entwicklung im Jahre } 1998 \text { (in ATS) gegliedert } \\
\text { nach Wissenschaftszweigen und Ausgabenarten }\end{array}$ \\
\hline 17 & $\begin{array}{l}\text { Hochschulsektor: Finanzierung der Ausgaben für Forschung und experimentelle Entwicklung im Jahre } 1998 \text { (in } \\
\text { ATS) gegliedert nach Wissenschaftszweigen und Finanzierungsbereichen }\end{array}$ \\
\hline 18 & $\begin{array}{l}\text { Sektor Staat: Ausgaben für Forschung und experimentelle Entwicklung im Jahre } 1998 \text { (in ATS) gegliedert nach } \\
\text { Wissenschaftszweigen und Ausgabenarten }\end{array}$ \\
\hline 19 & $\begin{array}{l}\text { Sektor Staat: Finanzierung der Ausgaben für Forschung und experimentelle Entwicklung im Jahre } 1998 \text { (in } \\
\text { ATS) gegliedert nach Wissenschaftszweigen und Finanzierungsbereichen }\end{array}$ \\
\hline 20 & $\begin{array}{l}\text { Sektor Staat: Ausgaben für Forschung und experimentelle Entwicklung im Jahre } 1998 \text { (in ATS) gegliedert nach } \\
\text { Rechtsträgern und Ausgabenarten }\end{array}$ \\
\hline 21 & $\begin{array}{l}\text { Sektor Staat: Finanzierung der Ausgaben für Forschung und experimentelle Entwicklung im Jahre } 1998 \text { (in } \\
\text { ATS) gegliedert nach Rechtsträgern und Finanzierungsbereichen }\end{array}$ \\
\hline 22 & $\begin{array}{l}\text { Privater gemeinnütziger Sektor: Ausgaben für Forschung und experimentelle Entwicklung im Jahre } 1998 \text { (in } \\
\text { ATS) gegliedert nach Wissenschaftszweigen und Ausgabenarten }\end{array}$ \\
\hline 23 & $\begin{array}{l}\text { Privater gemeinnütziger Sektor: Finanzierung der Ausgaben für Forschung und experimentelle Entwicklung im } \\
\text { Jahre } 1998 \text { (in ATS) gegliedert nach Wissenschaftszweigen und Finanzierungsbereichen }\end{array}$ \\
\hline 24 & $\begin{array}{l}\text { Unternehmenssektor ohne Firmeneigener Bereich: Ausgaben für Forschung und experimentelle Entwicklung } \\
\text { im Jahre } 1998 \text { (in ATS) gegliedert nach Wissenschaftszweigen und Ausgabenarten }\end{array}$ \\
\hline 25 & $\begin{array}{l}\text { Unternehmenssektor ohne Firmeneigener Bereich: Finanzierung der Ausgaben für Forschung und } \\
\text { experimentelle Entwicklung im Jahre } 1998 \text { (in ATS) gegliedert nach Wissenschaftszweigen und } \\
\text { Finanzierungsbereichen }\end{array}$ \\
\hline 26 & $\begin{array}{l}\text { Forschung und experimentelle Entwicklung in sämtlichen Durchführungssektoren (ohne Firmeneigener } \\
\text { Bereich): Ausgaben für F\&E im Jahre } 1998 \text { (in ATS) gegliedert nach Wissenschaftszweigen und } \\
\text { Ausgabenarten }\end{array}$ \\
\hline 27 & $\begin{array}{l}\text { Forschung und experimentelle Entwicklung in sämtlichen Durchführungssektoren (ohne Firmeneigener } \\
\text { Bereich): Finanzierung der Ausgaben für F\&E im Jahre } 1998 \text { (in ATS) gegliedert nach Wissenschaftszweigen } \\
\text { und Finanzierungsbereichen }\end{array}$ \\
\hline 28 & $\begin{array}{l}\text { Forschung und experimentelle Entwicklung in sämtlichen Durchführungssektoren (einschl. Firmeneigener } \\
\text { Bereich): Ausgaben für F\&E im Jahre } 1998 \text { (in ATS) nach Durchführungssektoren/Erhebungsbereichen und } \\
\text { Ausgabenarten }\end{array}$ \\
\hline 29 & $\begin{array}{l}\text { Forschung und experimentelle Entwicklung in sämtlichen Durchführungssektoren (einschl. Firmeneigener } \\
\text { Bereich): Finanzierung der Ausgaben für F\&E im Jahre } 1998 \text { (in ATS) gegliedert nach } \\
\text { Durchführungssektoren/Erhebungsbereichen und Finanzierungsbereichen }\end{array}$ \\
\hline 30 & $\begin{array}{l}\text { Forschung und experimentelle Entwicklung in sämtlichen Durchführungssektoren einschl. Firmeneigener } \\
\text { Bereich): Ausgaben für F\&E im Jahre } 1998 \text { (in ATS) gegliedert nach Bundesländern und Ausgabenarten }\end{array}$ \\
\hline 31 & $\begin{array}{l}\text { Forschung und experimentelle Entwicklung in sämtlichen Durchführungssektoren (ohne Firmeneigener } \\
\text { Bereich): Beschäftigte in F\&E (in Kopfzahlen) im Jahre } 1998 \text { nach } \\
\text { Durchführungssektoren/Erhebungsbereichen und sozio-ökonomischen Zielsetzungen }\end{array}$ \\
\hline 32 & $\begin{array}{l}\text { Forschung und experimentelle Entwicklung in sämtlichen Durchführungssektoren (ohne Firmeneigener } \\
\text { Bereich): Beschäftigte in F\&E (in Vollzeitäquivalenten) im Jahre } 1998 \text { nach } \\
\text { Durchführungssektoren/Erhebungsbereichen und sozio-ökonomischen Zielsetzungen }\end{array}$ \\
\hline 33 & $\begin{array}{l}\text { Forschung und experimentelle Entwicklung in sämtlichen Durchführungssektoren (ohne Firmeneigener } \\
\text { Bereich): Ausgaben für F\&E im Jahre } 1998 \text { nach Durchführungssektoren/Erhebungsbereichen und sozio- } \\
\text { ökonomischen Zielsetzungen }\end{array}$ \\
\hline
\end{tabular}




\begin{tabular}{|c|l|}
\hline 34 & Forschung und experimentelle Entwicklung (F\&E) 1999 im internationalen Vergleich \\
\hline 35 & FWF: Bewilligungen nach Forschungsstätten 2001(Mio. €) \\
\hline 36 & FWF: Bewilligungen nach Forschungsstätten 2001: Zahl der Neubewilligungen \\
\hline 37 & FWF: Förderungskategorien 2001: Neu- und Zusatzbewilligungen in Mio. € \\
\hline 38 & FWF: Bewilligungen nach Wissenschaftsdisziplinen 1999 - 2001 (Mio. €) \\
\hline 39 & FFF: Förderungsübersicht 2001 nach Systematik der Wirtschaftstätigkeit \\
\hline 40 & FFF: Förderungsübersicht 2001 nach Sonderbereichen der Forschung \\
\hline 41 & FFF: Förderungsübersicht 2001 nach Bundesländern (Projektstandort) \\
\hline
\end{tabular}


Globalschätzung 2002: Bruttoinlandsausgaben für F\&E

Finanzierung der in Österreich durchgeführten Forschung und experimentellen Entwicklung 1981 - 2002

\begin{tabular}{|c|c|c|c|c|c|c|c|c|c|c|c|c|c|c|c|c|c|}
\hline & Finanzierungssektoren & 1981 & 1985 & 1989 & 1990 & 1991 & 1992 & 1993 & 1994 & 1995 & 1996 & 1997 & 1998 & 1999 & 2000 & 2001 & 2002 \\
\hline \multirow[t]{7}{*}{1.} & Bruttoinlandsausgaben für & & & & & & n Milli & onen $\mathrm{E}$ & U RO & & & & & & & & \\
\hline & $\begin{array}{l}\text { F\&E } \\
\text { Davon finanziert durch: }\end{array}$ & 896,14 & $1.248,68$ & $1.669,07$ & $1.857,58$ & $2.104,78$ & $2.203,55$ & $2.303,31$ & $2.550,73$ & $2.700,67$ & $2.884,05$ & $3.121,60$ & $3.399,83$ & $3.655,96$ & $3.805,69$ & $4.030,89$ & $4.217,27$ \\
\hline & A. Bund ${ }^{1}$ ) & 362,40 & 518,17 & 617,84 & 695,33 & 836,04 & 893,50 & 957,12 & $1.075,14$ & $1.092,28$ & $1.066,46$ & $1.077,59$ & $1.097,51$ & $1.200,82$ & $1.225,42$ & $1.356,92$ & $1.456,64$ \\
\hline & B. Bundesländer ${ }^{2}$ ) & 47,86 & 71,20 & 89,38 & 108,66 & 123,68 & 133,57 & 129,67 & 158,69 & 153,89 & 159,06 & 167,35 & 142,41 & 205,25 & 244,13 & 260,04 & 267,80 \\
\hline & C. Unternehmenssektor ${ }^{3}$ ) & 450,20 & 613,35 & 885,35 & 967,79 & $1.057,61$ & $1.086,69$ & $1.128,40$ & $1.179,42$ & $1.233,49$ & $1.290,76$ & $1.352,58$ & $1.418,43$ & $1.472,33$ & $1.529,75$ & $1.587,88$ & $1.645,05$ \\
\hline & D. Ausland ${ }^{4}$ ) & 22,17 & 30,90 & 53,87 & 58,02 & 62,14 & 65,94 & 59,69 & 106,52 & 189,10 & 335,50 & 476,61 & 684,63 & 718,53 & 745,16 & 762,91 & 782,87 \\
\hline & E. Sonstige ${ }^{5}$ ) & 13,51 & 15,07 & 22,63 & 27,79 & 25,31 & 23,85 & 28,42 & 30,96 & 31,91 & 32,27 & 47,47 & 56,86 & 59,03 & 61,23 & 63,14 & 64,91 \\
\hline \multirow[t]{2}{*}{2.} & BIP nominell ${ }^{6}$ ) (in Mrd. & & & & & & & & & & & & & & & & \\
\hline & EURO) & 79,62 & 100,77 & 123,48 & 133,60 & 143,23 & 151,83 & 156,94 & 165,41 & 172,29 & 178,05 & 182,49 & 189,94 & 196,66 & 204,84 & 210,70 & 215,74 \\
\hline
\end{tabular}

1. Bruttoinlandsausgaben für F\&E

\section{in Millionen ATS}

Davon finanziert durch:
A. Bund ${ }^{1}$ )
B. Bundesländer ${ }^{2}$ )
C. Unternehmenssektor ${ }^{3}$ )
D. Ausland ${ }^{4}$ )

E. Sonstige ${ }^{5}$ )

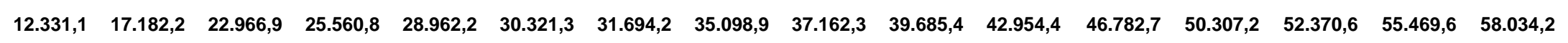

2

BIP nominell ${ }^{6}$ ) (in Mrd. ATS)

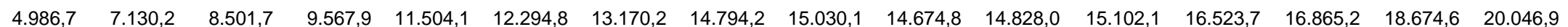

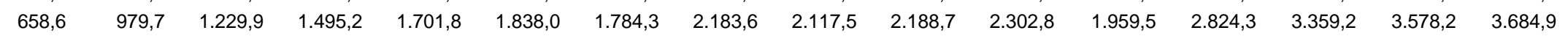

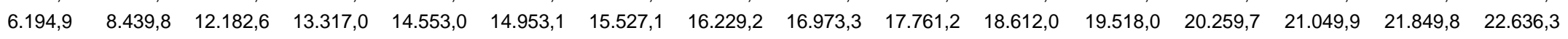

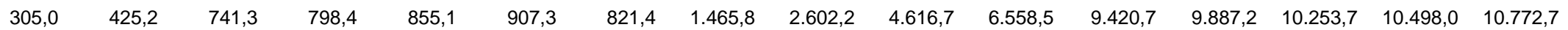

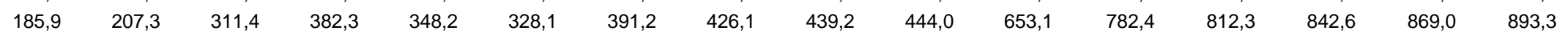

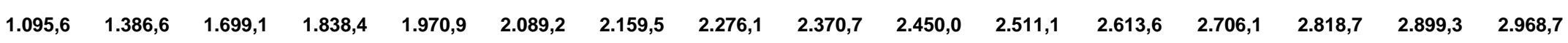

3. Bruttoinlandsausgaben für F\&E

1,13

1,24

1,35

1,39

1,47

1,45

1,47

1,54

1,57

1,62

1,71

1,79

1,86

Quelle: Statistik Austria (Bundesanstalt Statistik Österreich)

Stand: April 2002

Fußnoten ${ }^{1}$ ) bis ${ }^{6}$ ) siehe umseitig, Tabelle 1a! 
Globalschätzung 2002: Bruttoinlandsausgaben für F\&E

Finanzierung der in Österreich durchgeführten Forschung und experimentellen Entwicklung 1981 - 2002 (in Prozent des BIP)

\begin{tabular}{|c|c|c|c|c|c|c|c|c|c|c|c|c|c|c|c|c|c|}
\hline & Finanzierungssektoren & 1981 & 1985 & 1989 & 1990 & 1991 & 1992 & 1993 & 1994 & 1995 & 1996 & 1997 & 1998 & 1999 & 2000 & 2001 & 2002 \\
\hline \multirow[t]{8}{*}{1.} & Bruttoinlandsausgaben für & & & & & & & & & & & & & & & & \\
\hline & F\&E & 1,13 & 1,24 & 1,35 & 1,39 & 1,47 & 1,45 & 1,47 & 1,54 & 1,57 & 1,62 & 1,71 & 1,79 & 1,86 & 1,86 & 1,91 & 1,95 \\
\hline & Davon finanziert durch: & & & & & & & & & & & & & & & & \\
\hline & A. Bund ${ }^{1}$ ) & 0,46 & 0,51 & 0,50 & 0,52 & 0,58 & 0,59 & 0,61 & 0,65 & 0,63 & 0,60 & 0,59 & 0,58 & 0,61 & 0,60 & 0,64 & 0,68 \\
\hline & B. Bundesländer ${ }^{2}$ ) & 0,06 & 0,07 & 0,07 & 0,08 & 0,09 & 0,09 & 0,08 & 0,10 & 0,09 & 0,09 & 0,09 & 0,07 & 0,10 & 0,12 & 0,12 & 0,12 \\
\hline & C. Unternehmenssektor ${ }^{3}$ ) & 0,57 & 0,61 & 0,72 & 0,72 & 0,74 & 0,72 & 0,72 & 0,71 & 0,72 & 0,72 & 0,74 & 0,75 & 0,75 & 0,75 & 0,75 & 0,76 \\
\hline & D. Ausland ${ }^{4}$ ) & 0,03 & 0,03 & 0,04 & 0,04 & 0,04 & 0,04 & 0,04 & 0,06 & 0,11 & 0,19 & 0,26 & 0,36 & 0,37 & 0,36 & 0,36 & 0,36 \\
\hline & E. Sonstige ${ }^{5}$ ) & 0,02 & 0,01 & 0,02 & 0,02 & 0,02 & 0,02 & 0,02 & 0,02 & 0,02 & 0,02 & 0,03 & 0,03 & 0,03 & 0,03 & 0,03 & 0,03 \\
\hline \multirow[t]{2}{*}{2.} & BIP nominell ${ }^{6}$ ) & & & & & & & & & & & & & & & & \\
\hline & (in Mrd. EURO) & 79,62 & 100,77 & 123,48 & 133,60 & 143,23 & 151,83 & 156,94 & 165,41 & 172,29 & 178,05 & 182,49 & 189,94 & 196,66 & 204,84 & 210,70 & 215,74 \\
\hline & BIP nominell ${ }^{6}$ ) & & & & & & & & & & & & & & & & \\
\hline & (In Mird & $1.095,6$ & $1.386,6$ & $1.699,1$ & $1.838,4$ & $1.970,9$ & $2.089,2$ & $2.159,5$ & $2.276,1$ & $2.370,7$ & $2.450,0$ & $2.511,1$ & $2.613,6$ & $2.706,1$ & $2.818,7$ & $2.899,3$ & $2.968,7$ \\
\hline
\end{tabular}

1) 1981, 1985, 1989, 1993 und 1998: Erhebungsergebnisse (Bund einschließlich Mittel der zwei Forschungsförderungsfonds sowie ab 1989 auch einschließlich des ITF).

1990 - 1992, 1994 - 1997 und 1999 - 2002: Beilage T/Teil b (Bundesbudget-Forschung); 1990 zusätzlich Sonderaktion zur Förderung außenhandelsorientierter F\&E-Vorhaben (50,0 Mio.ATS).

999 und 2000: Einschließlich jener Rücklagenreste aus den Technologiemilliarden 1997 und 1998, die in den Jahren 1999 und 2000 für Forschungszwecke in Anspruch genommen wurden.

2001 und 2002: Im Bundesbudget Forschung sind für 2001 unter dem VA-Ansatz 1/5182 12 für das F\&E-Offensivprogramm 508,7 Mio EURO (7 Mrd. ATS) veranschlagt. Aufgrund der bisher vorliegenden Informationen wurden von diesen 508,7 Mio EURO (7 Mrd. ATS) im Jahre 2001 ca. 130,2 Mio. EURO (1,791 Mrd. ATS) ausgegeben. Für 2002 wird von der Annahme ausgegangen, daß ca. 218 Mio. EURO (3,0 Mrd. ATS) ausgegeben werden, die Inanspruchnahme der restlichen Mittel (ca. 160 Mio. EURO bzW. 2,2 Mrd. ATS) ist für F\&E-Ausgaben des Jahres 2003 vorgesehen. In die F\&E-Ausgaben-Schätzung von Statistik Austria sind daher - abweichend von der Beilage T/Teil b - nur jene Mittel, welche im Jahre 2001 ausgegeben wurden bzw. 2002 der Forschung zufließen sollen, eingegangen.

2) 1981, 1985, 1989, 1993 und 1998: Erhebungsergebnisse (einschließlich Schätzung der F\&E-Ausgaben der Landeskrankenanstalten durch Statistik Austria: 1981: 27,3 Mio. EURO bzw. 375,9 Mio. ATS; 1985: 37,1 Mio. EURO bzw. 510,9 Mio. ATS; 1989: 46,3 Mio EURO bzw. 637,7 Mio. ATS; 1993: 65,6 Mio EURO bzw. 903,1 Mio. ATS, 1998: 74,5 Mio EURO bzw. 1.025,4 Mio. ATS).

1990 - 1992, 1994 bis 1997 und 1999 - 2002: Schätzung durch Statistik Austria unter Heranziehung der F\&E-Ausgaben-Schätzungen der Ämter der Landesregierungen.

${ }^{3}$ ) Umfaßt Finanzierung durch die Wirtschaft (einschl. Jubiläumsfonds der Oesterreichischen Nationalbank).

981, 1985, 1989, 1993 und 1998: Erhebungsergebnisse.

1990 - 1992, 1994 - 1997 und 1999 - 2002: Schätzung durch Statistik Austria auf der Basis der Ergebnisse der F\&E-Erhebungen der Wirtschaftskammer Österreich (1989, 1991 und 1993$)$ und von Statistik Austria (1989, 1993 und 1998 Ergebnisse der F\&E-Erhebung von Statistik Austria im firmeneigenen Bereich).

4) 1981, 1985, 1989, 1993 und 1998: Erhebungsergebnisse. 1990 - 1992, 1994 - 1997 und 1999 - 2002: Schätzung durch Statistik Austria unter Berücksichtigung der Ergebnisse der F\&E-Erhebung 1998 von Statistik Austria. 1995 - 2000 unter Einschluß der Rückflüsse aus dem 4. EU-Rahmenprogramm für Forschung, technologische Entwicklung und Demonstration sowie ab 1999 bis 2002 unter Einschluß der Rückflüsse aus dem 5. EU-Rahmenprogramm für Forschung, technologische Entwicklung und Demonstration (Stand: April 2002).

5) Umfaßt Finanzierung durch Gemeinden (ohne Wien), durch Kammern, durch Sozialversicherungsträger sowie allfällige sonstige öffentliche Finanzierung (schließt auch von 1989 bis einschließlich 1998 im Wege der ASFINAG, sowie 1993 bis einschließlich 2000 durch die BIG außerbudgetär finanzierte Bauvorhaben im Hochschulsektor mit ein) und durch den privaten gemeinnützigen Sektor.

1981, 1985, 1989, 1993 und 1998: Erhebungsergebnisse. 1990 - 1992, 1994 - 1997 und 1999 - 2002: Schätzung durch Statistik Austria.

6) 1981 - 2000: Statistik Austria; 2001, 2002: WIFO-Prognose (April 2002). 
Tabelle 2: Ausgaben des Bundes für Forschung und Forschungsförderung 1999 bis 2002 nach Ressorts

Aufgliederung der Beilage T der Amtsbehelfe zu den Bundesfinanzgesetzen 2001 und 2002 (Teil a und Teil b)

\begin{tabular}{|c|c|c|c|c|c|c|c|c|}
\hline \multirow{3}{*}{ Ressorts ${ }^{1}$ ) } & \multicolumn{4}{|c|}{ Erfolg } & \multicolumn{4}{|c|}{ Bundesvoranschlag } \\
\hline & $\left.1999^{2}\right)$ & & $\left.2000^{3}\right)$ & & $\left.\left.2001^{3}\right)^{5}\right)$ & & $\left.2002^{3}\right)$ & \\
\hline & Mio. $€$ & $\%$ & Mio. $€$ & $\%$ & Mio. $€$ & $\%$ & Mio. $€$ & $\%$ \\
\hline Bundeskanzleramt & 7,875 & 0,6 & 5,246 & 0,4 & 5,126 & 0,3 & 4,880 & 0,4 \\
\hline Bundesministerium für Inneres & 0,162 & 0,0 & 0,146 & 0,0 & 0,145 & 0,0 & 0,091 & 0,0 \\
\hline Bundesministerium für Unterricht und kulturelle Angelegenheiten ${ }^{4}$ ) & 37,237 & 2,9 & . & . & . & & . & \\
\hline Bundesministerium für Wissenschaft und Verkehr ${ }^{4}$ ) & 939,525 & 73,4 & . & . & . & & $\cdot$ & \\
\hline Bundesministerium für Bildung, Wissenschaft und Kultur ${ }^{4}$ ) & & . & 982,312 & 76,3 & 975,384 & 54,8 & 974,117 & 76,0 \\
\hline Bundesministerium für Arbeit, Gesundheit und Soziales & 148,382 & 11,6 & . & . & . & & . & \\
\hline Bundesministerium für soziale Sicherheit und Generationen & . & . & 35,811 & 2,8 & 10,603 & 0,6 & 10,685 & 0,8 \\
\hline Bundesministerium für Umwelt, Jugend und Familie & 9,935 & 0,8 & . & . & . & & . & \\
\hline Bundesministerium für auswärtige Angelegenheiten & 1,822 & 0,1 & 2,063 & 0,2 & 2,004 & 0,1 & 1,993 & 0,2 \\
\hline Bundesministerium für Justiz & 0,073 & 0,0 & 0,062 & 0,0 & 0,062 & 0,0 & 0,073 & 0,0 \\
\hline Bundesministerium für Landesverteidigung & 0,190 & 0,0 & 0,263 & 0,0 & 0,258 & 0,0 & 0,267 & 0,0 \\
\hline Bundesministerium für Finanzen ${ }^{5}$ ) & 30,210 & 2,4 & 32,185 & 2,5 & 542,237 & 30,5 & 49,937 & 3,9 \\
\hline Bundesministerium für Land- und Forstwirtschaft ${ }^{4}$ ) & 37,753 & 2,9 & . & . & . & & . & \\
\hline Bundesministerium für Land- und Forstwirtschaft, Umwelt und Wasserwirtschaft $\left.{ }^{4}\right)^{6}$ ) & & . & 47,546 & 3,7 & 45,857 & 2,6 & 44,080 & 3,4 \\
\hline Bundesministerium für wirtschaftliche Angelegenheiten ${ }^{7}$ ) & 68,334 & 5,3 & . & . & . & & $\cdot$ & \\
\hline Bundesministerium für Wirtschaft und Arbeit $^{7}$ ) & . & . & 16,704 & 1,3 & 7,538 & 0,4 & 7,799 & 0,6 \\
\hline Bundesministerium für Verkehr, Innovation und Technologie & . & . & 164,988 & 12,8 & 190,067 & 10,7 & 189,011 & 14,7 \\
\hline Bundesministerium für öffentliche Leistung und Sport & . & . & - & - & - & - & - & \\
\hline
\end{tabular}

\section{INSGESAMT}

$1281,498 \quad 100,0$

$1287,326 \quad 100,0$

$1779,281 \quad 100,0$

$1282,933 \quad 100,0$

Stand: April 2002

Quelle: Statistik Austria (Bundesanstalt Statistik Österreich)

1) Entsprechend der im jeweiligen Jahr gültigen Fassung des Bundesministeriengesetzes 1986 (1999: BGBI. I Nr. 21/1997; 2000, 2001, 2002: BGBI. I Nr. 16/2000).

2) Amtsbehelf zum Bundesfinanzgesetz 2001. Revidierte Daten.

3) Amtsbehelf zum Bundesfinanzgesetz 2002.

4) Einschließlich der VA-Ansätze aus Kapitel 64 für Bauausgaben für dem Bundesministerium unterstehende F\&E ausführende Bundeseinrichtungen.

5) Bundesvoranschlag 2001: Einschließlich der gesamten im BFG 2001 rücklagefähig veranschlagten Mittel der Forschungs- und Technologieoffensive (508,7 Mio. $€$ ).

$\left.{ }^{6}\right)$ Erfolg 2000: Einschließlich der im Budgetkapitel 18 veranschlagten Ausgaben für Forschung und Forschungsförderung im Bereich Umwelt.

7) Ohne die VA-Ansätze aus Kapitel 64 für Bauausgaben für einem anderen Bundesministerium unterstehende F\&E ausführende Bundeseinrichtungen. 


\section{Tabelle 3}

\section{BEILAGE T}

\section{des Amtsbehelfes zum Bundesfinanzgesetz 2002}

\section{Forschungswirksame Ausgaben des Bundes von 2000 bis 2002 nach Ressorts}

Die nachfolgenden Übersichten für die Jahre 2000 bis 2002 sind aufgegliedert nach

1. Beitragszahlungen aus Bundesmitteln an internationale Organisationen, die Forschung und Forschungsförderung (mit) als Ziel haben (Teil a)

2. sonstigen Ausgaben des Bundes für Forschung und Forschungsförderung (Teil b, Bundesbudget Forschung)

Für die Aufstellung dieser Ausgaben ist in erster Linie der Gesichtspunkt der Forschungswirksamkeit maßgebend, der inhaltlich über den Aufgabenbereich 12 „Forschung und Wissenschaft" hinausgeht und auf dem Forschungsbegriff des FrascatiHandbuches der OECD beruht, wie er im Rahmen der forschungsstatistischen Erhebungen von STATISTIK AUSTRIA zur Anwendung gelangt.

Forschungswirksame Anteile bei den Bundesausgaben finden sich daher nicht nur bei den Ausgaben des Aufgabenbereiches 12 „Forschung und Wissenschaft", sondern auch in zahlreichen anderen Aufgabenbereichen (z. B. 11/Erziehung und Unterricht, 13/Kunst, 34/Land- und Forstwirtschaft, 36/Industrie und Gewerbe, 43/Übrige Hoheitsverwaltung), bei denen die Zielsetzungen des betreffenden Aufgabenbereiches im Vordergrund stehen.

Zur Beachtung: Die Anmerkungen zu den nachfolgenden Übersichten finden sich im Anhang zur Beilage $T$. 
Beilage $T$

Forschungswirksame Ausgaben des Bundes $\left({ }^{*}\right)$ (Beträge in Millionen Euro)

a) Beitragszahlungen aus Bundesmitteln an internationale Organisationen, die Forschung und Forschungsförderung (mit) als Ziel haben

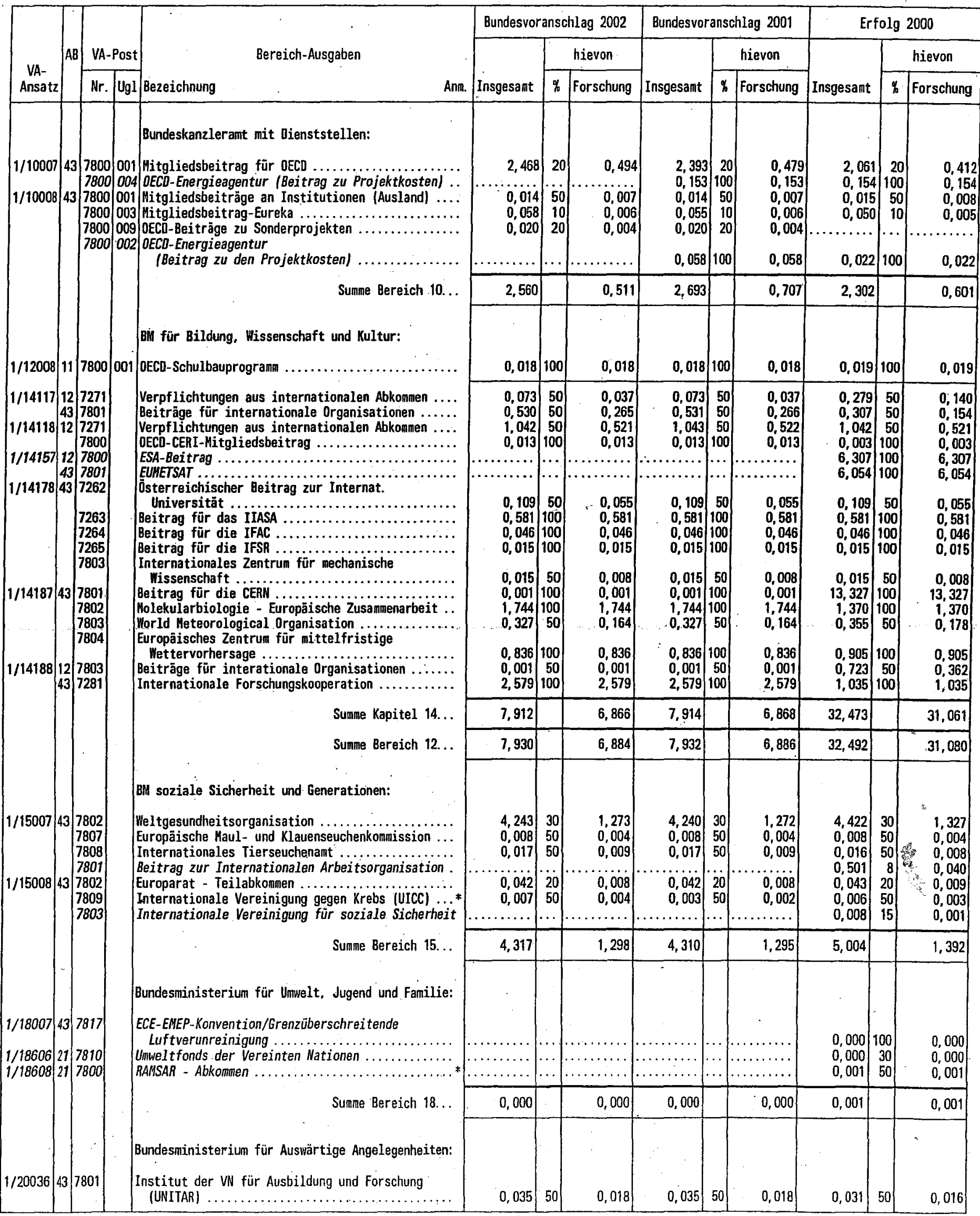


a) Beitragszahlungen aus Bundesmitteln an internationale Organisationen, die Forschung und Forschungsförderung (mit) als Ziel haben

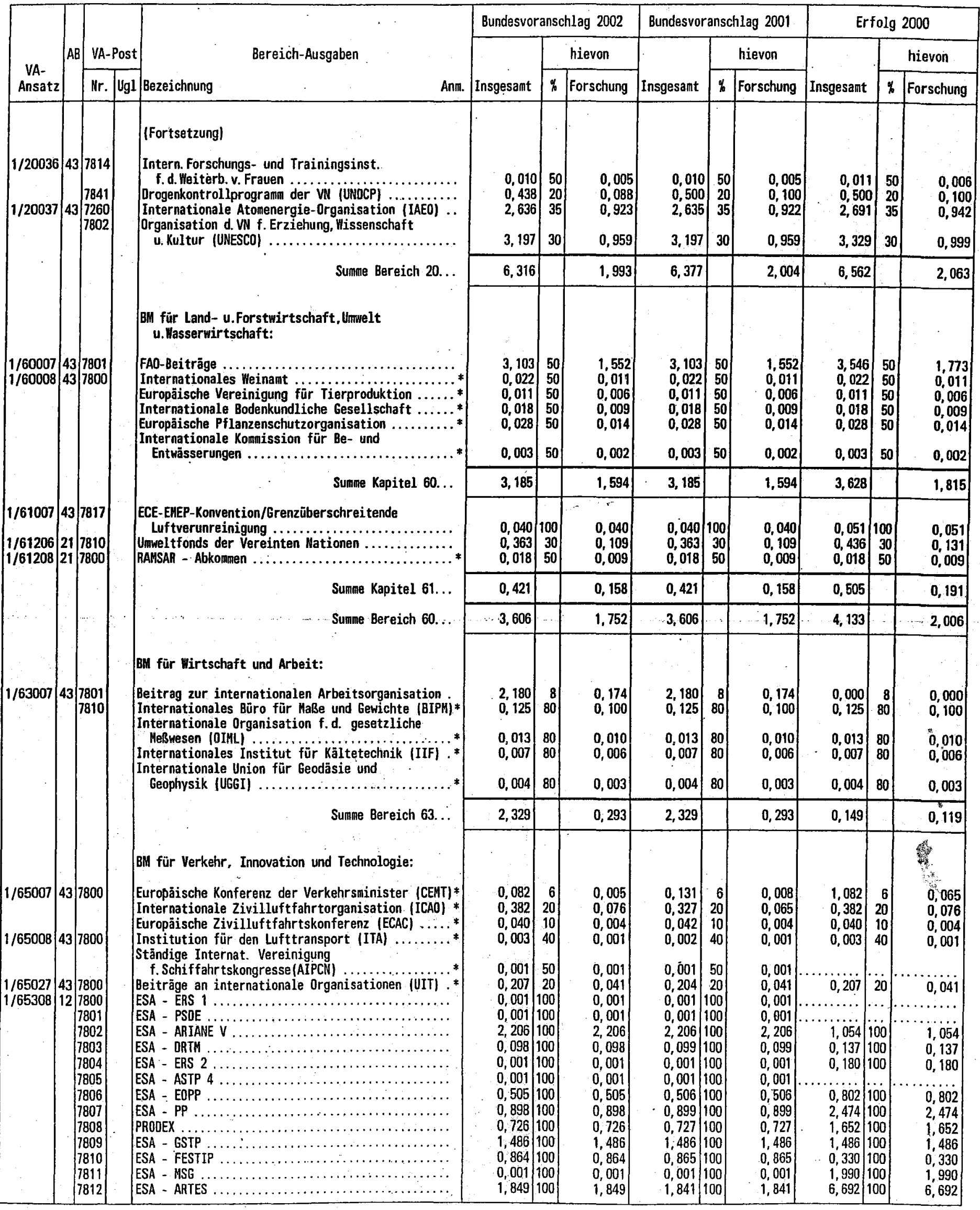


Forschungswirksame Ausgaben des Bundes $(*)$ (Beträge in Millionen Euro)

a) Beitragszahlungen aus Bundesmitteln an internationale Organisationen, die Forschung und Forschungsförderung (mit) als Ziel haben

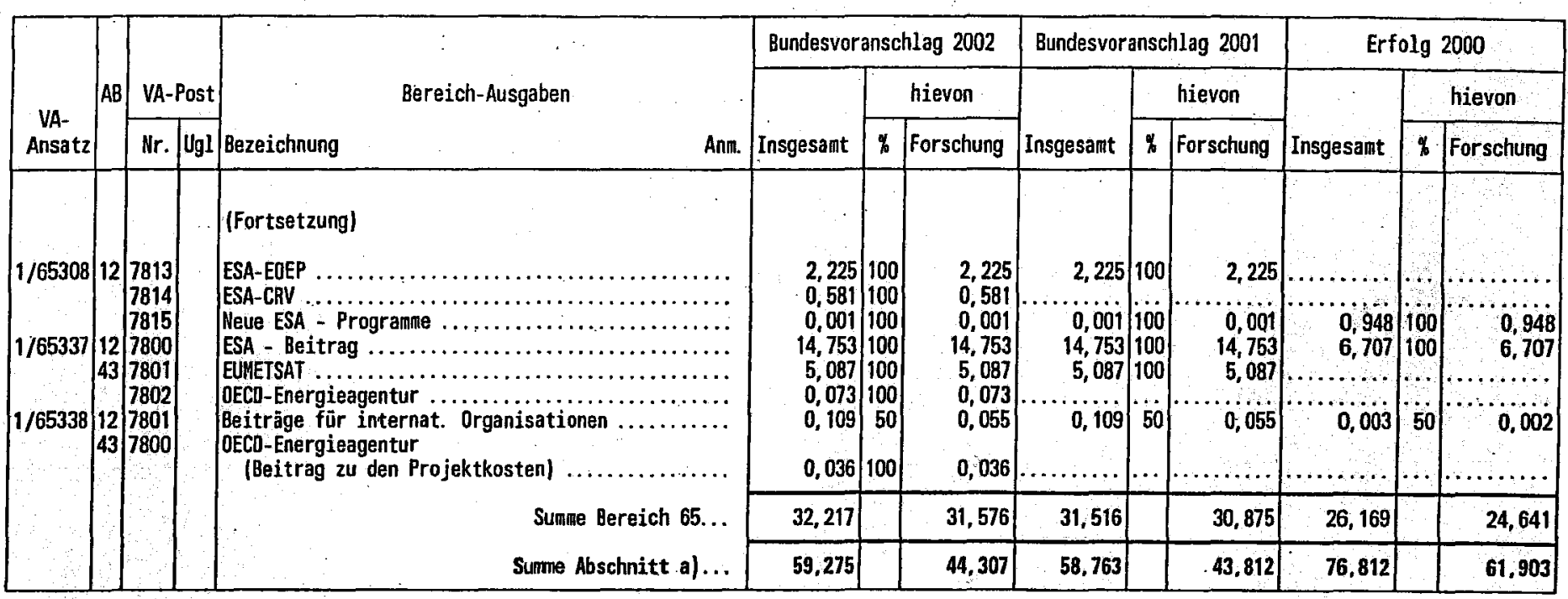


b) Ausgaben des Bundes (ausgen. die bereits im Abschnitt a) ausgewiesen sind) für Forschung und Forschungsförderung (Bundesbudget-Forschung)

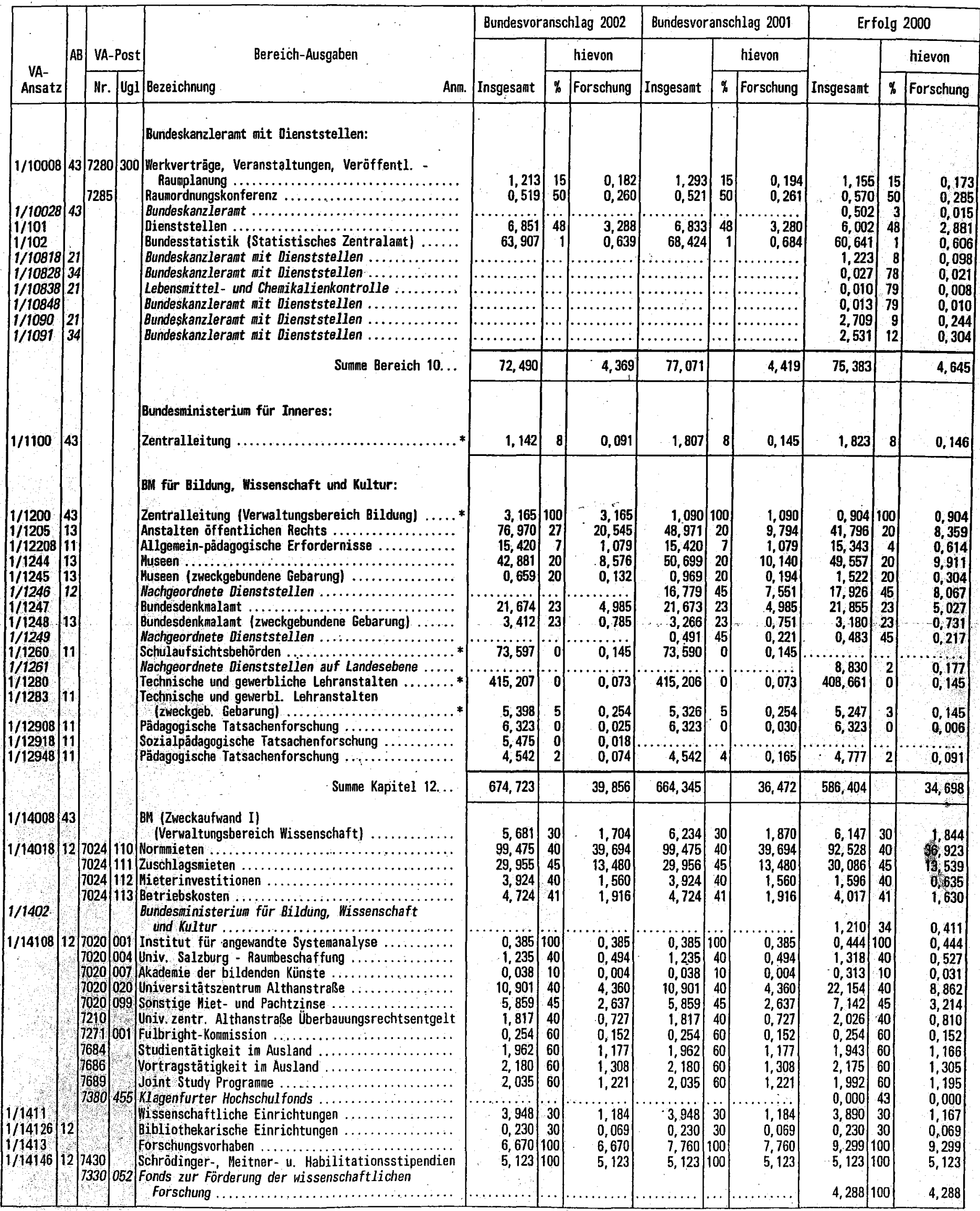


b) Ausgaben des Bundes (ausgen. die bereits im Abschnitt a) ausgewiesen sind) für Forschung und Forschungsförderung (Bundesbudget-Forschung)

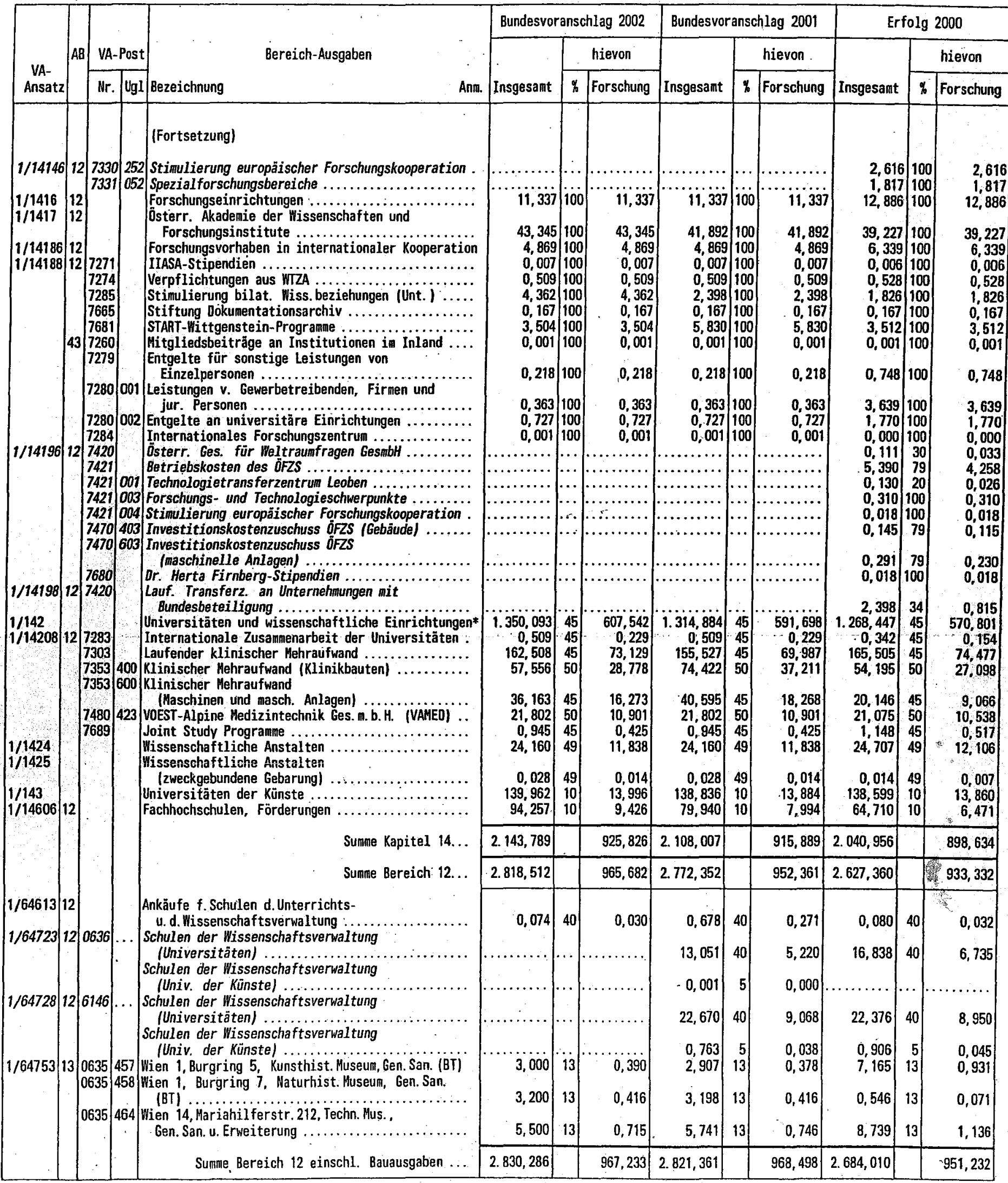


b) Ausgaben des Bundes (ausgen. die bereits in Abschnitt a) ausgewiesen sind) für Forschung und Forschungsförderung (Bundesbudget-Forschung)

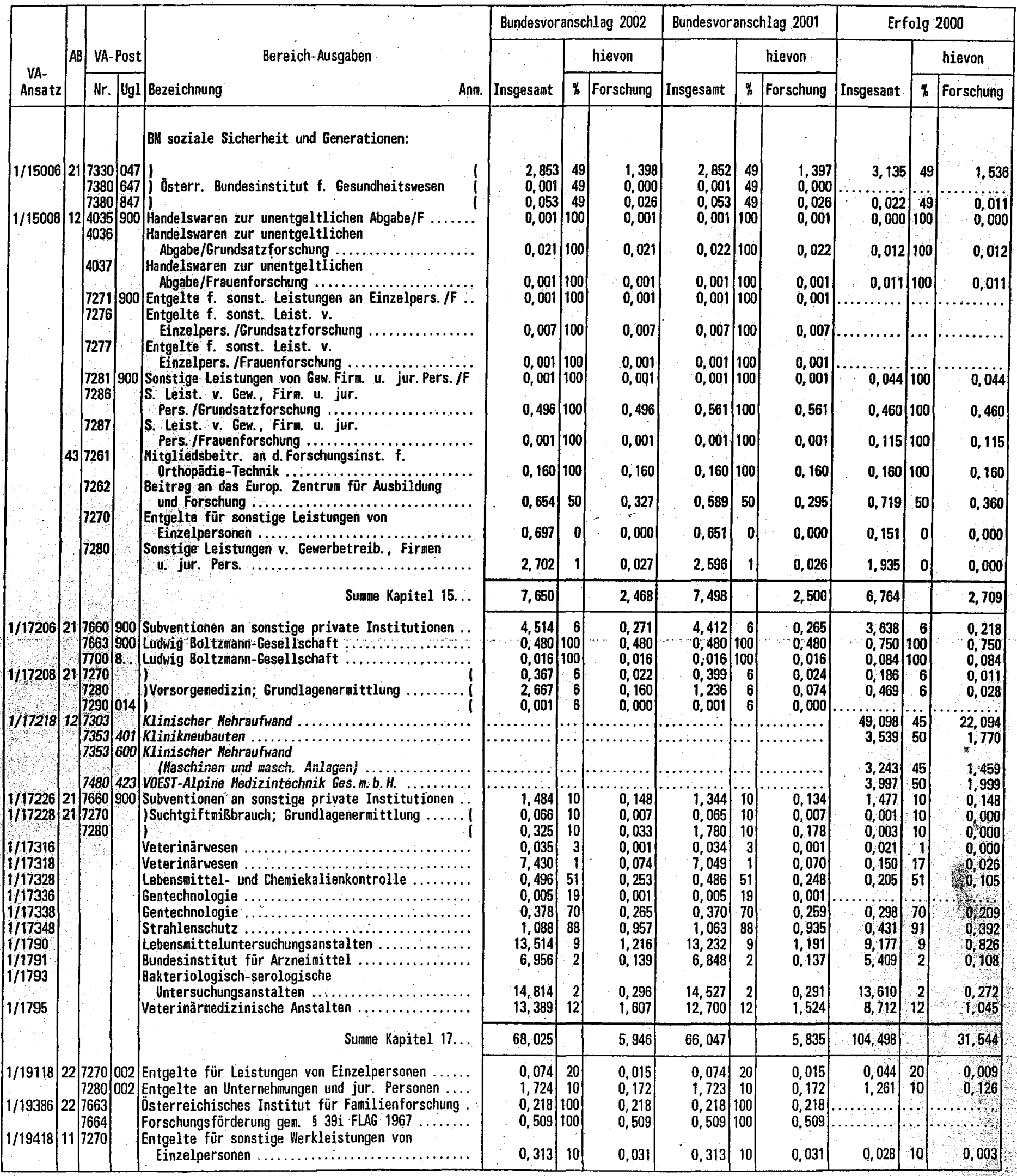


b) Ausgaben des Bundes (ausgen. die bereits im Abschnitt a) ausgewiesen sind) für Forschung und Forschungsfördẹrung (Bundesbudget-Forschung)

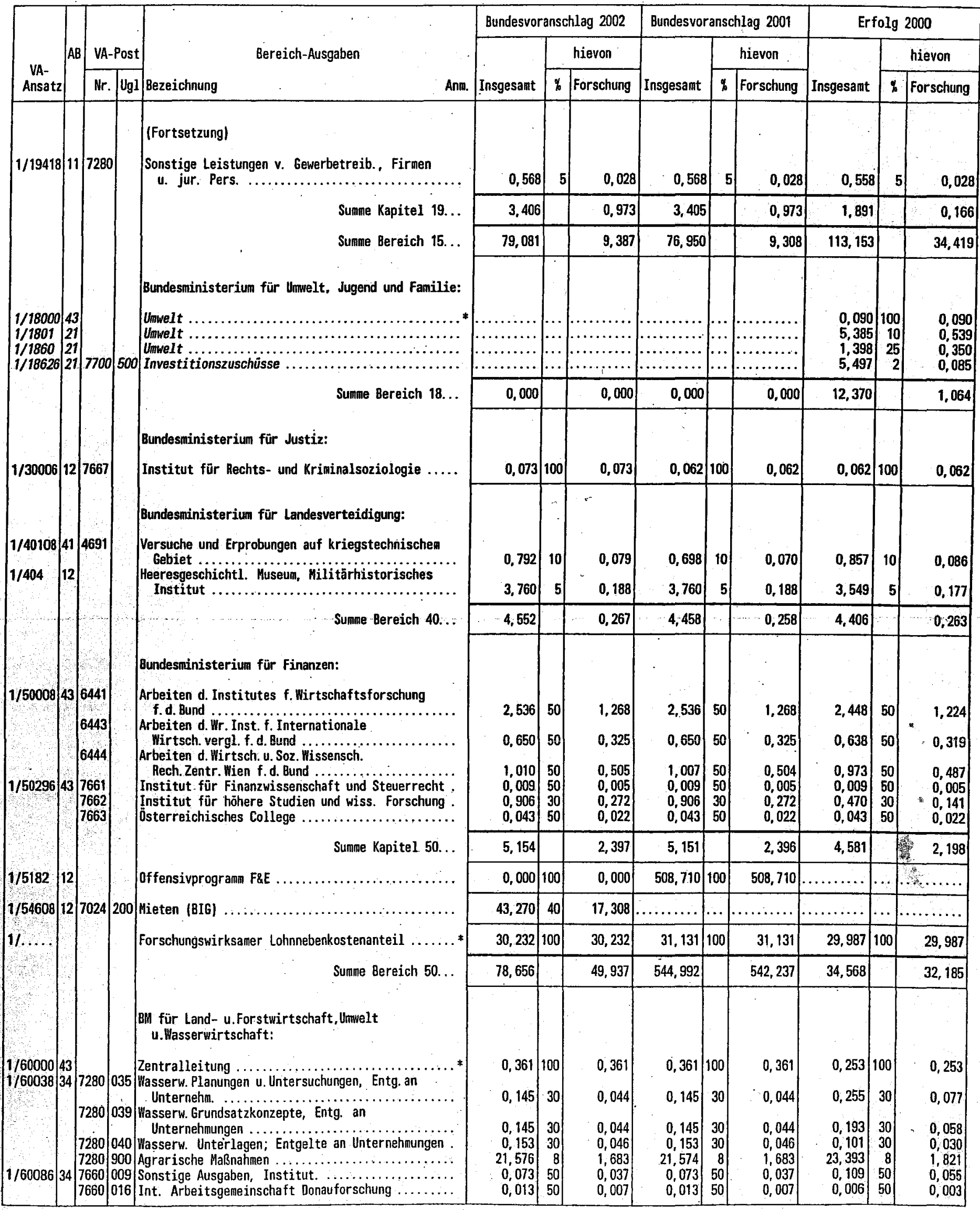


b) Ausgaben des Bundes (ausgen. die bereits im Abschnitt a) ausgewiesen sind) für Forschung und Forschungsförderung (Bundesbudget-Forschung)

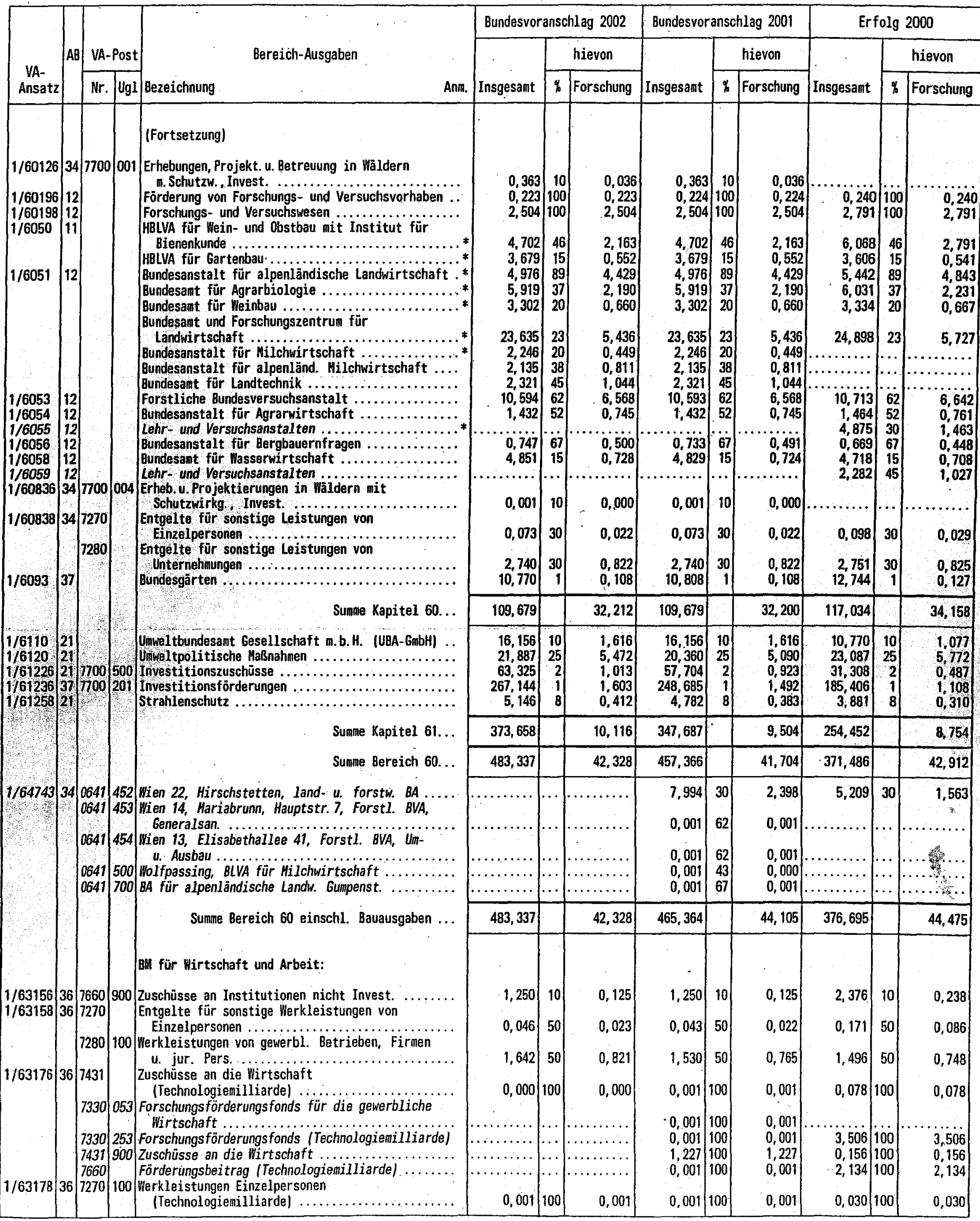


b) Ausgaben des Bundes (ausgen. die bereits im Abschnitt a) ausgewiesen sind) für Forschung und Forschungsförderung (Bundesbudget-Forschung)

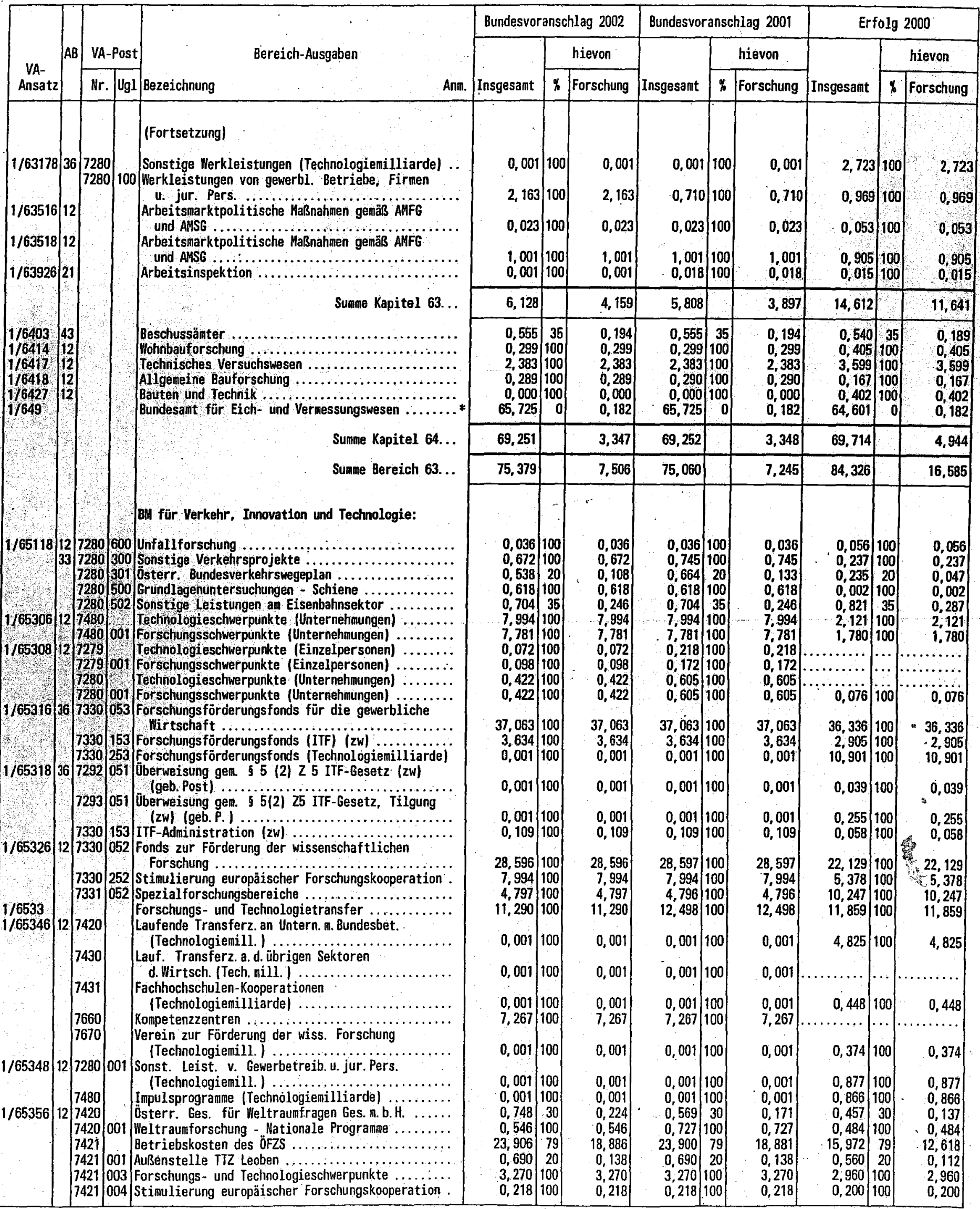


b) Ausgaben des Bundes (ausgen. die bereits im Abschnitt a) ausgewiesen sind) für Forschung und Forschungsförderung (Bundesbudget-Forschung)

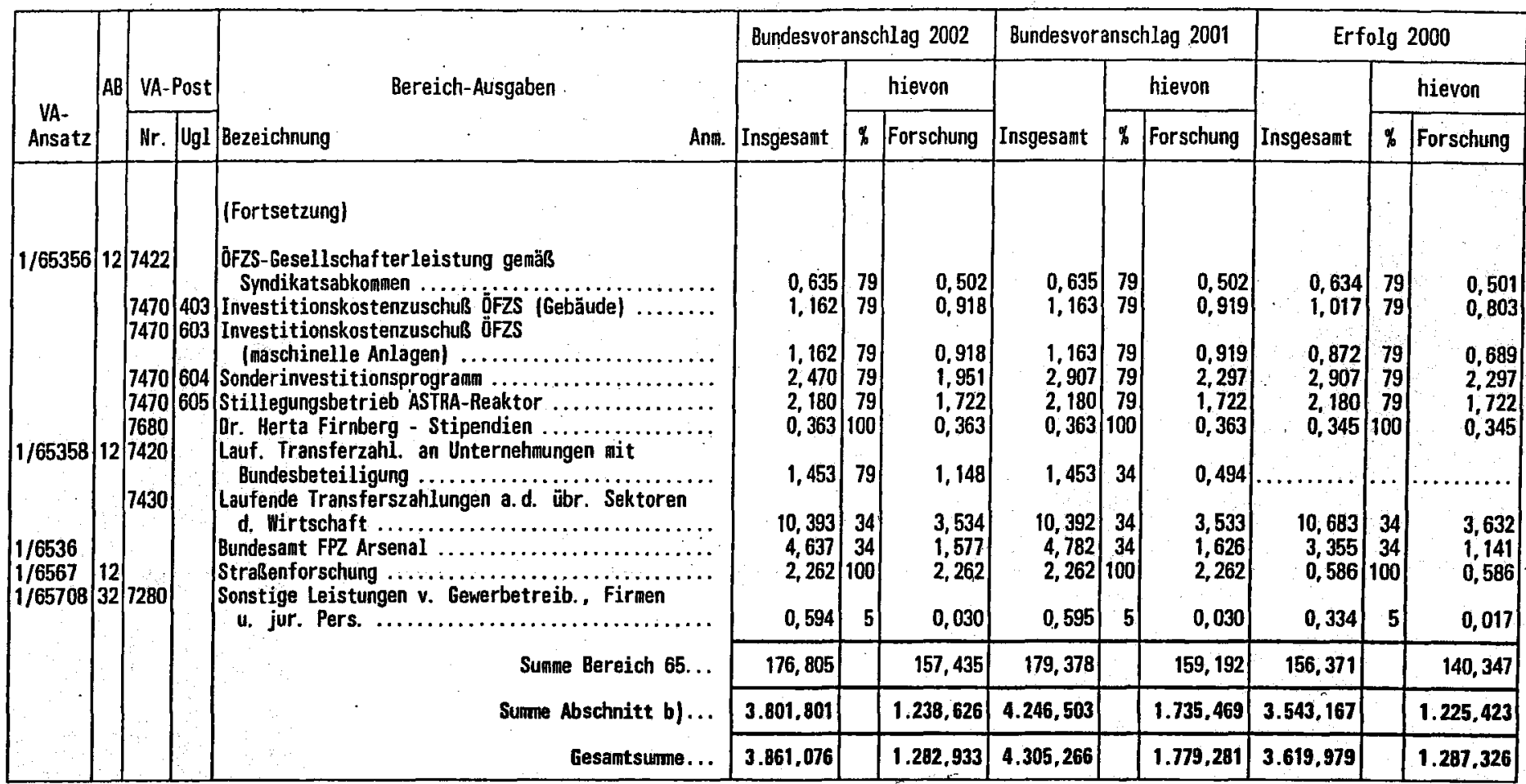


*) F \& E Koeffizienten geschätzt

Die Beilage $T$ ist aufgegliedert nach:

a) Beitragszahlungen aus Bundesmitteln an internationale Organisationen, die Forschung und Forschungsförderung (mit) als Ziel haben,

b) sonstigen Ausgaben des Bundes für Forschung und Forschungsförderung (Bundesbudget-Forschung)

Für die Aufstellung dieser Ausgaben ist in erster Linie der Gesichtspunkt der Forschungswirksamkeit maßgebend, der inhaltlich über den Aufgabenbereich 12 'Forschung und Wissenschaft' hinausgeht und auf dem Forschungsbegriff des Frascati-Handbuches der OECD beruht, wie er im Rahmen der forschungsstatistischen Erhebungen des OSTAT zur Anwendung gelangt.

Forschungswirksame Anteile bei den Bundesausgaben finden sich daher nicht nur bei den Ausgaben des Aufgabenbereiches 12 'Forschung und thissenschaft' sondern auch in zahlreichen anderen Aufgabenbereichen (z. B. 11/Erziehung und Unterricht, 13/Kunst, 34/Land und Forstwirtschaft, 36/Industrie und Gewerbe, 43/Übrige Hoheitsverwaltung), bei denen die Zielsetzungen des betreffenden Aufgabenbereiches in Vordergrund stehen.

VA- $\rightarrow$ VA-Post

Ansatz AB Mr. Ugl A n n e r k u g

$1 / 110043$

$1 / 120043$

$1 / 1260$

$1 / 1280$

$1 / 128311$

$1 / 142$

$1 / 15008437809$

$1 / 1800043$

$1 / 18608217800$

$1 / 6000043$

$1 / 60008437800$

$1 / 6050 \quad 11$

$1 / 6051 \quad 12$

$1 / 6055 \quad 12$

$1 / 61208217800$

$1 / 649$

$1 / 65007437800$

$1 / 65008437800$

$1 / 65027437800$

$1 / \ldots$
Teilbetráge verschiedener VA-Posten beim VA-Ansatz 1/11000 und 1/11003 sowie VA-Posten 4006/001, 4552, 4572, 4592, 6182 und 7252 beim VA-Ansatz $1 / 11008$.

Forschungsanteil . Pauschalbetrag

Forschungsanteil: Pauschalbetrag

Forschungsanteil: Pauschalbetrag.

Forschungsanteil. Pauschalbetrag

Ohne Ausgaben der unterhalb angeführten VA-Posten $1 / 142 . \ldots \ldots \ldots$

Teilbetrag der VA-Post.

Teilbetrag des VA-Ansatzes.

Teilbetrag der VA-Post.

Teilbetrag des VA-Ansatzes.

Teilbetrag der VA-Post.

Von den übrigen landwirtschaftlichen Bundeslehranstalten werden Forschungs- und Versuchsaufgaben derzeit nicht durchgeführt.

Teilbeträge verschiedener VA-Posten.

Ohne Ausgaben der VA-Posten 7150,7151 des VA-Ansatzes 1/60557 und der VA-Fosten 4030, 4251 des VA-Ansatzes 1 160558 .

Ohne Aus

Teilbetrag der VA-Post.

Forschungsanteil: Pauschalbetrag.

Teilbetrag der. VA-Post.

Teilbetrag der VA-Post.

Teilbetrag der VA-Post.

F\&E-Anteil an den Lohnnebenkosten der in Forschungseinrichtungen tätigen Bundesbeamten. Imputation nach OECD-Aichtlinien. 
Tabelle 4: $\quad$ Ausgaben des Bundes 1993 bis 2002 für Forschung und Forschungsförderung nach sozio-ökonomischen Zielsetzungen (ÖSTAT-Systematik)

Aufgliederung der Beilage T des Amtsbehelfes zum jeweiligen Bundesfinanzgesetz (Teil a und Teil b)

\begin{tabular}{|c|c|c|c|c|c|c|c|c|c|c|c|c|c|c|c|}
\hline \multirow{2}{*}{\multicolumn{2}{|c|}{ Berichtsjahre }} & \multirow[b]{2}{*}{$\begin{array}{c}\text { Ausgaben } \\
\text { für F\&E } \\
\text { insgesamt }\end{array}$} & \multicolumn{13}{|c|}{ davon für } \\
\hline & & & \begin{tabular}{|c|} 
Förderung der \\
Erfor- \\
schung der \\
Erde, der \\
Meere, der \\
Atmos- \\
phäre und \\
des Welt- \\
raumes
\end{tabular} & $\begin{array}{l}\text { Förderung } \\
\text { der Land- } \\
\text { und Forst- } \\
\text { wirtschaft }\end{array}$ & $\begin{array}{c}\text { Förderung } \\
\text { von Handel, } \\
\text { Gewerbe } \\
\text { und } \\
\text { Industrie }\end{array}$ & $\begin{array}{c}\text { Förderung der } \\
\text { Erzeugung, } \\
\text { Speiche- } \\
\text { rung und } \\
\text { Verteilung } \\
\text { von Energie }\end{array}$ & $\begin{array}{c}\text { Förderung } \\
\text { des Transport-, } \\
\text { Verkehrs- } \\
\text { und Nach- } \\
\text { richten- } \\
\text { wesens }\end{array}$ & $\begin{array}{c}\text { Förderung des } \\
\text { Unterrichts- } \\
\text { und Bildungs- } \\
\text { wesens }\end{array}$ & $\begin{array}{c}\text { Förderung des } \\
\text { Gesund- } \\
\text { heits- } \\
\text { wesens }\end{array}$ & $\begin{array}{c}\text { Förderung der } \\
\text { sozialen } \\
\text { und sozio- } \\
\text { ökonomi- } \\
\text { schen Ent- } \\
\text { wicklung }\end{array}$ & $\begin{array}{c}\text { Förderung des } \\
\text { Umwelt- } \\
\text { schutzes }\end{array}$ & \begin{tabular}{|} 
Förderung der \\
Stadt- \\
und \\
Raum- \\
planung
\end{tabular} & $\begin{array}{l}\text { Förderung } \\
\text { der } \\
\text { Landes- } \\
\text { vertei- } \\
\text { digung }\end{array}$ & $\begin{array}{c}\text { Förderung } \\
\text { anderer } \\
\text { Zielset- } \\
\text { zungen }\end{array}$ & 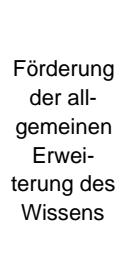 \\
\hline $\left.1993^{1}\right)$ & $\begin{array}{l}\text { in } 1000 € \\
\text { in } \%\end{array}$ & $\begin{array}{r}1063049 \\
100,0\end{array}$ & $\begin{array}{r}48743 \\
4,6\end{array}$ & $\begin{array}{r}48585 \\
4,6\end{array}$ & $\begin{array}{r}153961 \\
14,5\end{array}$ & $\begin{array}{r}18381 \\
1,7\end{array}$ & $\begin{array}{r}27194 \\
2,6\end{array}$ & $\begin{array}{r}14308 \\
1,3\end{array}$ & $\begin{array}{r}262368 \\
24,7\end{array}$ & $\begin{array}{r}69792 \\
6,6\end{array}$ & $\begin{array}{r}51015 \\
4,8\end{array}$ & $\begin{array}{r}6080 \\
0,6\end{array}$ & $\begin{array}{r}20 \\
0,0\end{array}$ & $\begin{array}{r}9353 \\
0,9\end{array}$ & $\begin{array}{r}353250 \\
33,1\end{array}$ \\
\hline $\left.1994^{2}\right)$ & $\begin{array}{l}\text { in } 1000 € \\
\text { in } \%\end{array}$ & $\begin{array}{r}1151932 \\
100,0\end{array}$ & $\begin{array}{r}50916 \\
4,4\end{array}$ & $\begin{array}{r}49590 \\
4,3\end{array}$ & $\begin{array}{r}177759 \\
15,4\end{array}$ & $\begin{array}{r}21797 \\
1,9\end{array}$ & $\begin{array}{r}36287 \\
3,2\end{array}$ & $\begin{array}{r}14997 \\
1,3\end{array}$ & $\begin{array}{r}273868 \\
23,8\end{array}$ & $\begin{array}{r}78242 \\
6,8\end{array}$ & $\begin{array}{r}52342 \\
4,5\end{array}$ & $\begin{array}{r}5747 \\
0,5\end{array}$ & $\begin{array}{l}137 \\
0,0\end{array}$ & $\begin{array}{r}10767 \\
0,9\end{array}$ & $\begin{array}{r}379484 \\
33,0\end{array}$ \\
\hline $\left.1995{ }^{3}\right)$ & $\begin{array}{l}\text { in } 1000 € \\
\text { in } \%\end{array}$ & $\begin{array}{r}1150418 \\
100,0\end{array}$ & $\begin{array}{r}55288 \\
4,8\end{array}$ & $\begin{array}{r}49073 \\
4,3\end{array}$ & $\begin{array}{r}169867 \\
14,8\end{array}$ & $\begin{array}{r}16869 \\
1,5\end{array}$ & $\begin{array}{r}32760 \\
2,8\end{array}$ & $\begin{array}{r}15350 \\
1,3\end{array}$ & $\begin{array}{r}270121 \\
23,5\end{array}$ & $\begin{array}{r}75571 \\
6,6\end{array}$ & $\begin{array}{r}47665 \\
4,1\end{array}$ & $\begin{array}{r}6531 \\
0,6\end{array}$ & $\begin{array}{r}82 \\
0,0\end{array}$ & $\begin{array}{r}11037 \\
1,0\end{array}$ & $\begin{array}{r}400206 \\
34,7\end{array}$ \\
\hline $\left.1996{ }^{4}\right)$ & $\begin{array}{l}\text { in } 1000 € \\
\text { in } \%\end{array}$ & $\begin{array}{r}1123669 \\
100,0\end{array}$ & $\begin{array}{r}54154 \\
4,8\end{array}$ & $\begin{array}{r}47560 \\
4,2\end{array}$ & $\begin{array}{r}163642 \\
14,6\end{array}$ & $\begin{array}{r}17052 \\
1,5\end{array}$ & $\begin{array}{r}28159 \\
2,5\end{array}$ & $\begin{array}{r}15488 \\
1,4\end{array}$ & $\begin{array}{r}248314 \\
22,1\end{array}$ & $\begin{array}{r}79359 \\
7,1\end{array}$ & 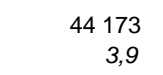 & $\begin{array}{r}6188 \\
0,6\end{array}$ & $\begin{array}{r}73 \\
0,0\end{array}$ & $\begin{array}{r}10856 \\
1,0\end{array}$ & $\begin{array}{r}408653 \\
36,3\end{array}$ \\
\hline $\left.19977^{5}\right)$ & $\begin{array}{l}\text { in } 1000 € \\
\text { in } \%\end{array}$ & $\begin{array}{r}1132901 \\
100,0\end{array}$ & $\begin{array}{r}54939 \\
4,8\end{array}$ & $\begin{array}{r}49177 \\
4,3\end{array}$ & $\begin{array}{r}155087 \\
13,7\end{array}$ & $\begin{array}{r}21884 \\
1,9\end{array}$ & $\begin{array}{r}30385 \\
2,7\end{array}$ & $\begin{array}{r}15713 \\
1,4\end{array}$ & $\begin{array}{r}265641 \\
23,4\end{array}$ & $\begin{array}{r}79076 \\
7,0\end{array}$ & $\begin{array}{r}43121 \\
3,8\end{array}$ & $\begin{array}{r}6433 \\
0,6\end{array}$ & $\begin{array}{r}31 \\
0,0\end{array}$ & $\begin{array}{r}11178 \\
1,0\end{array}$ & $\begin{array}{r}400236 \\
35,4\end{array}$ \\
\hline $1998{ }^{6}$ ) & $\begin{array}{l}\text { in } 1000 € \\
\text { in } \%\end{array}$ & $\begin{array}{r}1207908 \\
100,0\end{array}$ & $\begin{array}{r}85538 \\
7,1\end{array}$ & $\begin{array}{r}69262 \\
5,7\end{array}$ & $\begin{array}{r}173102 \\
14,3\end{array}$ & $\begin{array}{r}22694 \\
1,9\end{array}$ & $\begin{array}{r}34064 \\
2,8\end{array}$ & $\begin{array}{r}14514 \\
1,2\end{array}$ & $\begin{array}{r}270452 \\
22,4\end{array}$ & $\begin{array}{r}86414 \\
7,2\end{array}$ & $\begin{array}{r}41747 \\
3,5\end{array}$ & $\begin{array}{r}10090 \\
0,8\end{array}$ & $\begin{array}{r}57 \\
0,0\end{array}$ & $\begin{array}{r}11549 \\
1,0\end{array}$ & $\begin{array}{r}388424 \\
32,1\end{array}$ \\
\hline $\left.1999^{7}\right)$ & $\begin{array}{l}\text { in } 1000 € \\
\text { in } \%\end{array}$ & $\begin{array}{r}1281498 \\
100,0\end{array}$ & $\begin{array}{r}91387 \\
7,1\end{array}$ & $\begin{array}{r}75421 \\
5,9\end{array}$ & $\begin{array}{r}188151 \\
14,7\end{array}$ & $\begin{array}{r}25314 \\
2,0\end{array}$ & $\begin{array}{r}32337 \\
2,5\end{array}$ & $\begin{array}{r}15552 \\
1,2\end{array}$ & $\begin{array}{r}280577 \\
21,9\end{array}$ & $\begin{array}{r}91162 \\
7,1\end{array}$ & $\begin{array}{r}42771 \\
3,3\end{array}$ & $\begin{array}{r}10136 \\
0,8\end{array}$ & $\begin{array}{l}12 \\
0,0\end{array}$ & $\begin{array}{r}11348 \\
0,9\end{array}$ & $\begin{array}{r}417329 \\
32,6\end{array}$ \\
\hline $2000^{8}$ ) & $\begin{array}{l}\text { in } 1000 € \\
\text { in } \%\end{array}$ & $\begin{array}{r}1287326 \\
100,0\end{array}$ & $\begin{array}{r}86343 \\
6,7\end{array}$ & $\begin{array}{r}79177 \\
6,2\end{array}$ & $\begin{array}{r}194247 \\
15,1\end{array}$ & $\begin{array}{r}21365 \\
1,7\end{array}$ & $\begin{array}{r}29644 \\
2,3\end{array}$ & $\begin{array}{r}14299 \\
1,1\end{array}$ & $\begin{array}{r}291038 \\
22,6\end{array}$ & $\begin{array}{r}89881 \\
7,0\end{array}$ & $\begin{array}{r}43301 \\
3,4\end{array}$ & $\begin{array}{r}10006 \\
0,8\end{array}$ & $\begin{array}{l}336 \\
0,0\end{array}$ & $\begin{array}{r}11502 \\
0,9\end{array}$ & $\begin{array}{r}416187 \\
32,2\end{array}$ \\
\hline $\left.2001^{9}\right)^{10}$ ) & $\begin{array}{l}\text { in } 1000 € \\
\text { in } \%\end{array}$ & $\begin{array}{r}1400728 \\
100,0\end{array}$ & $\begin{array}{r}88344 \\
6,3\end{array}$ & $\begin{array}{r}79009 \\
5,6\end{array}$ & $\begin{array}{r}222224 \\
15,9\end{array}$ & $\begin{array}{r}22970 \\
1,6\end{array}$ & $\begin{array}{r}35034 \\
2,5\end{array}$ & $\begin{array}{r}14937 \\
1,1\end{array}$ & $\begin{array}{r}308653 \\
22,0\end{array}$ & $\begin{array}{r}91008 \\
6,5\end{array}$ & $\begin{array}{r}44273 \\
3,2\end{array}$ & $\begin{array}{r}10140 \\
0,7\end{array}$ & $\begin{array}{r}340 \\
0,0\end{array}$ & $\begin{array}{r}48855 \\
3,5\end{array}$ & $\begin{array}{r}434941 \\
31,1\end{array}$ \\
\hline $2002^{9}$ ) & $\begin{array}{l}\text { in } 1000 € \\
\text { in } \%\end{array}$ & $\begin{array}{r}1282933 \\
100,0\end{array}$ & $\begin{array}{r}85175 \\
6,6\end{array}$ & $\begin{array}{r}76410 \\
6,0\end{array}$ & $\begin{array}{r}195449 \\
15,2\end{array}$ & $\begin{array}{r}20327 \\
1,6\end{array}$ & $\begin{array}{r}34753 \\
2,7\end{array}$ & $\begin{array}{r}14975 \\
1,2\end{array}$ & $\begin{array}{r}295510 \\
23,0\end{array}$ & $\begin{array}{r}89751 \\
7,0\end{array}$ & $\begin{array}{r}44644 \\
3,5\end{array}$ & $\begin{array}{r}10141 \\
0,8\end{array}$ & $\begin{array}{l}357 \\
0,0\end{array}$ & $\begin{array}{r}11722 \\
0,9\end{array}$ & $\begin{array}{r}403719 \\
31,5\end{array}$ \\
\hline
\end{tabular}

\section{Stand: April 2002}

1) Beilage T des Amtsbehelfes zum BFG 1995, Erfolg. Revidierte Daten. 2. $)$ Beilage T des Amtsbehelfes zum BFG 1996, Erfolg. - ${ }^{3}$ ) Beilage T des Amtsbehelfes zum BFG 1997, Erfolg.

$\left.{ }^{4}\right)$ Beilage T des Amtsbehelfes zum BFG 1998, Erfolg. - ${ }^{5}$ ) Beilage T des Amtsbehelfes zum BFG 1999, Erfolg. . ${ }^{6}$ ) Beilage T des Amtsbehelfes zum BFG 2000, Erfolg. Revidierte Daten.

7) Beilage T des Amtsbehelfes zum BFG 2001, Erfolg. Revidierte Daten. ${ }^{-{ }^{8}}$ ) Beilage T des Amtsbehelfes zum BFG 2002, Erfolg. $-{ }^{9}$ ) Beilage T des Amtsbehelfes zum BFG 2002, Voranschlag.

Im Bundesbudget Forschung sind für 2001 unter dem VA-Ansatz $1 / 518212$ fur das F \& E-Offensivprogramm 508,7 Mio. $€$ (7 Mrd. ATS) veranschlagt. Aufgrund der bisher vorliegenden Informationen wird von der Annahme ausgegangen, dass von diesel 508,7 Mio. $€$ (7 Mrd. ATS) im Jahre 2001 nur ca. 130,2 Mio. $€$ (1,791 Mrd. ATS) ausgegeben wurden, die Inanspruchnahme der restlichen 378,553 Mio. $€(5,209$ Mrd. ATS) ist für F \& E-Ausgaben der Folgejahre vorgesehen. In die Auswertungen vo STATISTIK AUSTRIA sind daher - abweichend von der Beilage T/Teil b - nur jene 130,2 Mio. $€(1,791$ Mrd. ATS) eingegangen.

Rundungsdifferenzen 
Tabelle 5: Ausgaben des Bundes 1993 bis 2002 für Forschung und Forschungsförderung nach sozio-ökonomischen Zielsetzungen (ÖSTAT-Systematik) Aufgliederung der Beilage T des Amtsbehelfes zum jeweiligen Bundesfinanzgesetz (Teil a und Teil b)

\begin{tabular}{|c|c|c|c|c|c|c|c|c|c|c|c|c|c|c|c|}
\hline \multirow{2}{*}{\multicolumn{2}{|c|}{ Berichtsjahre }} & \multirow[b]{2}{*}{$\begin{array}{l}\text { Ausgaben } \\
\text { für F\&E } \\
\text { insgesamt }\end{array}$} & \multicolumn{13}{|c|}{ davon für } \\
\hline & & & \begin{tabular}{|c|} 
Förderung der \\
Erfor- \\
schung der \\
Erde, der \\
Meere, der \\
Atmos- \\
phäre und \\
des Welt- \\
raumes
\end{tabular} & $\begin{array}{l}\text { Förderung } \\
\text { der Land- } \\
\text { und Forst- } \\
\text { wirtschaft }\end{array}$ & $\begin{array}{c}\text { Förderung } \\
\text { von Handel, } \\
\text { Gewerbe } \\
\text { und } \\
\text { Industrie }\end{array}$ & \begin{tabular}{|c|} 
Förderung der \\
Erzeugung, \\
Speiche- \\
rung und \\
Verteilung \\
von Energie
\end{tabular} & $\begin{array}{c}\text { Förderung } \\
\text { des Transport } \\
, \\
\text { Verkehrs- } \\
\text { und Nach- } \\
\text { richten- } \\
\text { wesens }\end{array}$ & \begin{tabular}{|} 
Förderung des \\
Unterrichts- \\
und Bildungs- \\
wesens
\end{tabular} & $\begin{array}{c}\text { Förderung des } \\
\text { Gesund- } \\
\text { heits- } \\
\text { wesens }\end{array}$ & $\begin{array}{c}\text { Förderung der } \\
\text { sozialen } \\
\text { und sozio- } \\
\text { ökonomi- } \\
\text { schen Ent- } \\
\text { wicklung }\end{array}$ & $\begin{array}{c}\text { Förderung des } \\
\text { Umwelt- } \\
\text { schutzes }\end{array}$ & \begin{tabular}{|c|} 
Förderung der \\
Stadt- \\
und \\
Raum- \\
planung
\end{tabular} & $\begin{array}{l}\text { Förderung } \\
\text { der } \\
\text { Landes- } \\
\text { vertei- } \\
\text { digung }\end{array}$ & $\begin{array}{l}\text { Förderung } \\
\text { anderer } \\
\text { Zielset- } \\
\text { zungen }\end{array}$ & $\begin{array}{l}\text { Förderung } \\
\text { der all- } \\
\text { gemeinen } \\
\text { Erwei- } \\
\text { terung des } \\
\text { Wissens }\end{array}$ \\
\hline$\left(993^{1}\right)$ & $\begin{array}{l}\text { in } 1000 \mathrm{~S} \\
\text { in } \%\end{array}$ & $\begin{array}{r}14627873 \\
100,0\end{array}$ & $\begin{array}{r}670722 \\
4,6\end{array}$ & $\begin{array}{r}668543 \\
4,6\end{array}$ & $\begin{array}{r}2118552 \\
14,5\end{array}$ & $\begin{array}{r}252926 \\
1,7\end{array}$ & $\begin{array}{r}374198 \\
2,6\end{array}$ & $\begin{array}{r}196888 \\
1,3\end{array}$ & $\begin{array}{r}3610258 \\
24,7\end{array}$ & $\begin{array}{r}960353 \\
6,6\end{array}$ & $\begin{array}{r}701983 \\
4,8\end{array}$ & $\begin{array}{r}83656 \\
0,6\end{array}$ & $\begin{array}{r}270 \\
0,0\end{array}$ & $\begin{array}{r}128700 \\
0,9\end{array}$ & $\begin{array}{r}4860824 \\
33,1\end{array}$ \\
\hline $\left.1994{ }^{2}\right)$ & $\begin{array}{l}\text { in } 1000 \mathrm{~S} \\
\text { in } \%\end{array}$ & $\begin{array}{r}15850932 \\
100,0\end{array}$ & $\begin{array}{r}700616 \\
4,4\end{array}$ & $\begin{array}{r}682372 \\
4,3\end{array}$ & $\begin{array}{r}2446014 \\
15,4\end{array}$ & $\begin{array}{r}299935 \\
1,9\end{array}$ & $\begin{array}{r}499317 \\
3,2\end{array}$ & $\begin{array}{r}206366 \\
1,3\end{array}$ & $\begin{array}{r}3768506 \\
23,8\end{array}$ & $\begin{array}{r}1076635 \\
6,8\end{array}$ & $\begin{array}{r}720236 \\
4,5\end{array}$ & $\begin{array}{r}79083 \\
0,5\end{array}$ & $\begin{array}{r}1879 \\
0,0\end{array}$ & $\begin{array}{r}148155 \\
0,9\end{array}$ & $\begin{array}{r}5221818 \\
33,0\end{array}$ \\
\hline $\left.1995^{3}\right)$ & $\begin{array}{l}\text { in } 1000 \mathrm{~S} \\
\text { in } \%\end{array}$ & $\begin{array}{r}15830102 \\
100,0\end{array}$ & $\begin{array}{r}760773 \\
4,8\end{array}$ & $\begin{array}{r}675253 \\
4,3\end{array}$ & $\begin{array}{r}2337426 \\
14,8\end{array}$ & $\begin{array}{r}232126 \\
1,5\end{array}$ & $\begin{array}{r}450786 \\
2,8\end{array}$ & $\begin{array}{r}211215 \\
1,3\end{array}$ & $\begin{array}{r}3716947 \\
23,5\end{array}$ & $\begin{array}{r}1039879 \\
6,6\end{array}$ & $\begin{array}{r}655883 \\
4,1\end{array}$ & $\begin{array}{r}89867 \\
0,6\end{array}$ & $\begin{array}{r}1125 \\
0,0\end{array}$ & $\begin{array}{r}151871 \\
1,0\end{array}$ & $\begin{array}{r}5506951 \\
34,7\end{array}$ \\
\hline $\left.19966^{4}\right)$ & $\begin{array}{l}\text { in } 1000 \mathrm{~S} \\
\text { in } \%\end{array}$ & $\begin{array}{r}15462023 \\
100,0\end{array}$ & $\begin{array}{r}745171 \\
4,8\end{array}$ & $\begin{array}{r}654434 \\
4,2\end{array}$ & $\begin{array}{r}2251758 \\
14,6\end{array}$ & $\begin{array}{r}234639 \\
1,5\end{array}$ & $\begin{array}{r}387481 \\
2,5\end{array}$ & $\begin{array}{r}213113 \\
1,4\end{array}$ & $\begin{array}{r}3416878 \\
22,1\end{array}$ & $\begin{array}{r}1091998 \\
7,1\end{array}$ & $\begin{array}{r}607834 \\
3,9\end{array}$ & $\begin{array}{r}85150 \\
0,6\end{array}$ & $\begin{array}{r}999 \\
0,0\end{array}$ & $\begin{array}{r}149378 \\
1,0\end{array}$ & $\begin{array}{r}5623190 \\
36,3\end{array}$ \\
\hline $\left.1997^{5}\right)$ & $\begin{array}{l}\text { in } 1000 \mathrm{~S} \\
\text { in } \%\end{array}$ & $\begin{array}{r}15589061 \\
100,0\end{array}$ & $\begin{array}{r}755980 \\
4,8\end{array}$ & $\begin{array}{r}676693 \\
4,3\end{array}$ & $\begin{array}{r}2134047 \\
13,7\end{array}$ & $\begin{array}{r}301124 \\
1,9\end{array}$ & $\begin{array}{r}418113 \\
2,7\end{array}$ & $\begin{array}{r}216222 \\
1,4\end{array}$ & $\begin{array}{r}3655297 \\
23,4\end{array}$ & $\begin{array}{r}1088107 \\
7,0\end{array}$ & $\begin{array}{r}593357 \\
3,8\end{array}$ & $\begin{array}{r}88521 \\
0,6\end{array}$ & $\begin{array}{r}423 \\
0,0\end{array}$ & $\begin{array}{r}153814 \\
1,0\end{array}$ & $\begin{array}{r}5507363 \\
35,4\end{array}$ \\
\hline $\left.1998{ }^{6}\right)$ & $\begin{array}{l}\text { in } 1000 \mathrm{~S} \\
\text { in } \%\end{array}$ & $\begin{array}{r}16621182 \\
100,0\end{array}$ & $\begin{array}{r}1177034 \\
7,1\end{array}$ & $\begin{array}{r}953070 \\
5,7\end{array}$ & $\begin{array}{r}2381932 \\
14,3\end{array}$ & $\begin{array}{r}312272 \\
1,9\end{array}$ & $\begin{array}{r}468724 \\
2,8\end{array}$ & $\begin{array}{r}199723 \\
1,2\end{array}$ & $\begin{array}{r}3721501 \\
22,4\end{array}$ & $\begin{array}{r}1189081 \\
7,2\end{array}$ & $\begin{array}{r}574451 \\
3,5\end{array}$ & $\begin{array}{r}138847 \\
0,8\end{array}$ & $\begin{array}{r}790 \\
0,0\end{array}$ & $\begin{array}{r}158924 \\
1,0\end{array}$ & $\begin{array}{r}5344833 \\
32,1\end{array}$ \\
\hline $\left.1999^{7}\right)$ & $\begin{array}{l}\text { in } 1000 \mathrm{~S} \\
\text { in } \%\end{array}$ & $\begin{array}{r}17633796 \\
100,0\end{array}$ & $\begin{array}{r}1257516 \\
7,1\end{array}$ & $\begin{array}{r}1037822 \\
5,9\end{array}$ & $\begin{array}{r}2589020 \\
14,7\end{array}$ & $\begin{array}{r}348328 \\
2,0\end{array}$ & $\begin{array}{r}444966 \\
2,5\end{array}$ & $\begin{array}{r}214004 \\
1,2\end{array}$ & $\begin{array}{r}3860819 \\
21,9\end{array}$ & $\begin{array}{r}1254417 \\
7,1\end{array}$ & $\begin{array}{r}588538 \\
3,3\end{array}$ & $\begin{array}{r}139472 \\
0,8\end{array}$ & $\begin{array}{l}161 \\
0,0\end{array}$ & $\begin{array}{r}156156 \\
0,9\end{array}$ & $\begin{array}{r}5742577 \\
32,6\end{array}$ \\
\hline $2000^{8}$ ) & $\begin{array}{l}\text { in } 1000 \mathrm{~S} \\
\text { in } \%\end{array}$ & $\begin{array}{r}17713992 \\
100,0\end{array}$ & $\begin{array}{r}1188106 \\
6,7\end{array}$ & $\begin{array}{r}1089499 \\
6,2\end{array}$ & $\begin{array}{r}2672897 \\
15,1\end{array}$ & $\begin{array}{r}293989 \\
1,7\end{array}$ & $\begin{array}{r}407910 \\
2,3\end{array}$ & $\begin{array}{r}196759 \\
1,1\end{array}$ & $\begin{array}{r}4004770 \\
22,6\end{array}$ & $\begin{array}{r}1236790 \\
7,0\end{array}$ & $\begin{array}{r}595835 \\
3,4\end{array}$ & $\begin{array}{r}137686 \\
0,8\end{array}$ & $\begin{array}{r}4623 \\
0,0\end{array}$ & $\begin{array}{r}158271 \\
0,9\end{array}$ & $\begin{array}{r}5726858 \\
32,2\end{array}$ \\
\hline $\left.2001{ }^{9}\right)^{10}$ ) & $\begin{array}{l}\text { in } 1000 \mathrm{~S} \\
\text { in } \%\end{array}$ & $\begin{array}{r}19274437 \\
100,0\end{array}$ & $\begin{array}{r}1215640 \\
6,3\end{array}$ & $\begin{array}{r}1087188 \\
5,6\end{array}$ & $\begin{array}{r}3057869 \\
15,9\end{array}$ & $\begin{array}{r}316074 \\
1,6\end{array}$ & $\begin{array}{r}482078 \\
2,5\end{array}$ & $\begin{array}{r}205538 \\
1,1\end{array}$ & $\begin{array}{r}4247158 \\
22,0\end{array}$ & $\begin{array}{r}1252297 \\
6,5\end{array}$ & $\begin{array}{r}609210 \\
3,2\end{array}$ & $\begin{array}{r}139529 \\
0,7\end{array}$ & $\begin{array}{r}4679 \\
0,0\end{array}$ & $\begin{array}{r}672259 \\
3,5\end{array}$ & $\begin{array}{r}5984919 \\
31,1\end{array}$ \\
\hline $\left.2002^{9}\right)$ & $\begin{array}{l}\text { in } 1000 \mathrm{~S} \\
\text { in } \%\end{array}$ & $\begin{array}{r}17653543 \\
100,0\end{array}$ & $\begin{array}{r}1172034 \\
6,6\end{array}$ & $\begin{array}{r}1051425 \\
6,0\end{array}$ & $\begin{array}{r}2689437 \\
15,2\end{array}$ & $\begin{array}{r}279706 \\
1,6\end{array}$ & $\begin{array}{r}478212 \\
2,7\end{array}$ & $\begin{array}{r}206060 \\
1,2\end{array}$ & $\begin{array}{r}4066306 \\
23,0\end{array}$ & $\begin{array}{r}1235001 \\
7,0\end{array}$ & $\begin{array}{r}614315 \\
3,5\end{array}$ & $\begin{array}{r}139543 \\
0,8\end{array}$ & $\begin{array}{r}4912 \\
0,0\end{array}$ & $\begin{array}{r}161298 \\
0,9\end{array}$ & $\begin{array}{r}5555295 \\
31,5\end{array}$ \\
\hline
\end{tabular}

1) Beilage T des Amtsbehelfes zum BFG 1995, Erfolg. Revidierte Daten. - ${ }^{2}$ ) Beilage T des Amtsbehelfes zum BFG 1996, Erfolg. ${ }^{3}$ ) Beilage T des Amtsbehelfes zum BFG 1997, Erfolg. $\left.{ }^{4}\right)$ Beilage T des Amtsbehelfes zum BFG 1998, Erfolg. - ${ }^{5}$ ) Beilage T des Amtsbehelfes zum BFG 1999, Erfolg. - ${ }^{6}$ ) Beilage T des Amtsbehelfes zum BFG 2000, Erfolg. Revidierte Daten.

7) Beilage T des Amtsbehelfes zum BFG 2001, Erfolg. Revidierte Daten. ${ }^{8}$ ) Beilage T des Amtsbehelfes zum BFG 2002, Erfolg. ${ }^{-9}$ ) Beilage T des Amtsbehelfes zum BFG 2002, Voranschlag.

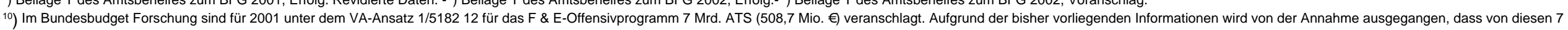
Mrd. ATS (508,7 Mio. €) im Jahre 2001 nur ca. 1,791 Mrd. ATS (130,2 Mio. €) ausgegeben wurden, die Inanspruchnahme der restlichen 5,209 Mrd. ATS (378,553 Mio. €) ist für F \& E-Ausgaben der Folgejahre vorgesehen. In die Auswertungen von STATISTIK AUSTRIA sind daher - abweichend von der Beilage T/Teil b - nur jene 1,791 Mrd. ATS (130,2 Mio. €) eingegangen.

Rundungsdifferenzen. 


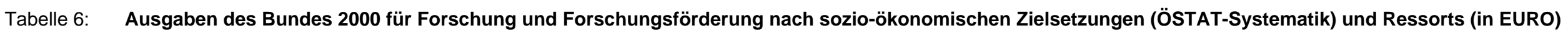
Aufgliederung der Jahreswerte $2000^{1}$ ) aus der Beilage T des Amtsbehelfes zum Bundesfinanzgesetz 2002 (Teil a und Teil b)

\begin{tabular}{|c|c|c|c|c|c|c|c|c|c|c|c|c|c|c|c|}
\hline \multirow{2}{*}{\multicolumn{2}{|c|}{ RESSORTS }} & \multirow[b]{2}{*}{$\begin{array}{c}\text { Ausgaben } \\
\text { des Bundes } \\
\text { für F\&E } \\
\text { insgesamt }\end{array}$} & \multicolumn{13}{|c|}{ davon für } \\
\hline & & & $\begin{array}{c}\text { Förderung } \\
\text { der Erfor- } \\
\text { schung der } \\
\text { Erde, der } \\
\text { Meere, der } \\
\text { Atmos- } \\
\text { phäre und } \\
\text { des Welt- } \\
\text { raumes }\end{array}$ & $\begin{array}{l}\text { Förderung } \\
\text { der Land- } \\
\text { und Forst- } \\
\text { wirtschaft }\end{array}$ & $\begin{array}{c}\text { Förderung } \\
\text { von Handel, } \\
\text { Gewerbe } \\
\text { und } \\
\text { Industrie }\end{array}$ & \begin{tabular}{|c|} 
Förderung \\
der \\
Erzeugung, \\
Speiche- \\
rung und \\
Verteilung \\
von Energie
\end{tabular} & $\begin{array}{c}\text { Förderung } \\
\text { des } \\
\text { Transport-, } \\
\text { Verkehrs- } \\
\text { und Nach- } \\
\text { richten- } \\
\text { wesens }\end{array}$ & \begin{tabular}{|c|} 
Förderung \\
des \\
Unterrichts- \\
und Bildungs \\
wesens
\end{tabular} & $\begin{array}{l}\text { Förderung } \\
\text { des } \\
\text { Gesund- } \\
\text { heits- } \\
\text { wesens }\end{array}$ & $\begin{array}{l}\text { Förderung } \\
\text { der } \\
\text { sozialen } \\
\text { und sozio- } \\
\text { ökonomi- } \\
\text { schen Ent- } \\
\text { wicklung }\end{array}$ & $\begin{array}{c}\text { Förderung } \\
\text { des } \\
\text { Umwelt- } \\
\text { schutzes }\end{array}$ & $\begin{array}{c}\text { Förderung } \\
\text { der Stadt- } \\
\text { und } \\
\text { Raum- } \\
\text { planung }\end{array}$ & $\begin{array}{c}\text { Förderung } \\
\text { der } \\
\text { Landes- } \\
\text { vertei- } \\
\text { digung }\end{array}$ & $\begin{array}{c}\text { Förderung } \\
\text { anderer } \\
\text { Zielset- } \\
\text { zungen }\end{array}$ & $\begin{array}{l}\text { Förderung } \\
\text { der all- } \\
\text { gemeinen } \\
\text { Erwei- } \\
\text { terung des } \\
\text { Wissens }\end{array}$ \\
\hline BKA & $\begin{array}{l}\text { in } 1000 € \\
\text { in } \%\end{array}$ & $\begin{array}{l}5246 \\
100,0\end{array}$ & - & - & $\begin{array}{r}5 \\
0,1\end{array}$ & $\begin{array}{r}176 \\
3,4\end{array}$ & - & - & $\begin{array}{r}262 \\
5,0\end{array}$ & $\begin{array}{r}1041 \\
19,8\end{array}$ & $\begin{array}{r}98 \\
1,9\end{array}$ & $\begin{array}{r}458 \\
8,7\end{array}$ & - & - & $\begin{array}{r}3206 \\
61,1\end{array}$ \\
\hline BMI & $\begin{array}{l}\text { in } 1000 € \\
\text { in } \%\end{array}$ & $\begin{array}{r}146 \\
100,0\end{array}$ & - & - & - & - & - & - & - & $\begin{array}{r}146 \\
100,0\end{array}$ & - & - & - & - & - \\
\hline BMBWK & $\begin{array}{l}\text { in } 1000 € \\
\text { in } \%\end{array}$ & $\begin{array}{r}982312 \\
100,0\end{array}$ & $\begin{array}{r}74676 \\
7,6\end{array}$ & $\begin{array}{r}42036 \\
4,3\end{array}$ & $\begin{array}{r}107423 \\
10,9\end{array}$ & $\begin{array}{r}14488 \\
1,5\end{array}$ & $\begin{array}{r}12340 \\
1,3\end{array}$ & $\begin{array}{r}13625 \\
1,4\end{array}$ & $\begin{array}{r}230571 \\
23,5\end{array}$ & $\begin{array}{r}77736 \\
7,9\end{array}$ & $\begin{array}{r}25265 \\
2,6\end{array}$ & $\begin{array}{r}8756 \\
0,9\end{array}$ & $\begin{array}{r}49 \\
0,0\end{array}$ & $\begin{array}{r}10815 \\
1,1\end{array}$ & $\begin{array}{r}364532 \\
37,0\end{array}$ \\
\hline BMSG & $\begin{array}{l}\text { in } 1000 € \\
\text { in } \%\end{array}$ & $\begin{array}{r}35811 \\
100,0\end{array}$ & - & $\begin{array}{r}12 \\
0,0\end{array}$ & - & - & $\begin{array}{l}- \\
-\end{array}$ & - & $\begin{array}{r}33118 \\
92,5\end{array}$ & $\begin{array}{r}1218 \\
3,4\end{array}$ & $\begin{array}{c}392 \\
1,1\end{array}$ & - & - & - & $\begin{array}{r}1071 \\
3,0\end{array}$ \\
\hline BMAA & $\begin{array}{l}\text { in } 1000 € \\
\text { in } \%\end{array}$ & $\begin{array}{l}2063 \\
100,0\end{array}$ & - & - & - & $\begin{array}{r}942 \\
45,7\end{array}$ & - & - & - & $\begin{array}{r}1105 \\
53,5\end{array}$ & - & - & - & - & $\begin{array}{r}16 \\
0,8\end{array}$ \\
\hline BMJ & $\begin{array}{l}\text { in } 1000 € \\
\text { in } \%\end{array}$ & $\begin{array}{r}62 \\
100,0\end{array}$ & - & - & - & - & - & - & - & $\begin{array}{r}62 \\
100,0\end{array}$ & - & - & - & - & - \\
\hline BML & $\begin{array}{l}\text { in } 1000 € \\
\text { in } \%\end{array}$ & $\begin{array}{r}263 \\
100,0\end{array}$ & - & - & - & - & - & - & - & - & - & - & $\begin{array}{r}86 \\
32,7\end{array}$ & - & $\begin{array}{r}177 \\
67,3\end{array}$ \\
\hline BMF & $\begin{array}{l}\text { in } 1000 € \\
\text { in } \%\end{array}$ & $\begin{array}{r}32185 \\
100,0\end{array}$ & $\begin{array}{r}2181 \\
6,8\end{array}$ & $\begin{array}{r}2216 \\
6,9\end{array}$ & $\begin{array}{r}3565 \\
11,1\end{array}$ & $\begin{array}{r}562 \\
1,7\end{array}$ & $\begin{array}{r}455 \\
1,4\end{array}$ & $\begin{array}{r}473 \\
1,5\end{array}$ & $\begin{array}{r}7211 \\
22,4\end{array}$ & $\begin{array}{r}4478 \\
13,9\end{array}$ & $\begin{array}{r}777 \\
2,4\end{array}$ & $\begin{array}{r}273 \\
0,8\end{array}$ & - & $\begin{array}{c}348 \\
1,1\end{array}$ & $\begin{array}{r}9646 \\
30,0\end{array}$ \\
\hline BMLFUW $^{2}$ ) & $\begin{array}{l}\text { in } 1000 € \\
\text { in } \%\end{array}$ & $\begin{array}{r}47546 \\
100,0\end{array}$ & $\begin{array}{r}565 \\
1,2\end{array}$ & $\begin{array}{r}33509 \\
70,5\end{array}$ & $\begin{array}{r}1530 \\
3,2\end{array}$ & - & - & - & - & $\begin{array}{r}1773 \\
3,7\end{array}$ & $\begin{array}{r}10093 \\
21,2\end{array}$ & - & - & - & $\begin{array}{r}76 \\
0,2\end{array}$ \\
\hline BMWA & $\begin{array}{l}\text { in } 1000 € \\
\text { in } \%\end{array}$ & $\begin{array}{r}16704 \\
100,0\end{array}$ & - & $\begin{array}{r}74 \\
0,4\end{array}$ & $\begin{array}{r}12229 \\
73,2\end{array}$ & $\begin{array}{l}473 \\
2,8\end{array}$ & $\begin{array}{r}1457 \\
8,7\end{array}$ & - & $\begin{array}{r}930 \\
5,6\end{array}$ & $\begin{array}{r}1054 \\
6,3\end{array}$ & $\begin{array}{r}79 \\
0,5\end{array}$ & $\begin{array}{r}81 \\
0,5\end{array}$ & - & - & $\begin{array}{l}327 \\
2,0\end{array}$ \\
\hline BMVIT & $\begin{array}{l}\text { in } 1000 € \\
\text { in } \%\end{array}$ & $\begin{array}{r}164988 \\
100,0\end{array}$ & $\begin{array}{r}8921 \\
5,4\end{array}$ & $\begin{array}{r}1330 \\
0,8\end{array}$ & $\begin{array}{r}69495 \\
42,1\end{array}$ & $\begin{array}{r}4724 \\
2,9\end{array}$ & $\begin{array}{r}15392 \\
9,3\end{array}$ & $\begin{array}{r}201 \\
0,1\end{array}$ & $\begin{array}{r}18946 \\
11,5\end{array}$ & $\begin{array}{r}1268 \\
0,8\end{array}$ & $\begin{array}{r}6597 \\
4,0\end{array}$ & $\begin{array}{r}438 \\
0,3\end{array}$ & $\begin{array}{l}201 \\
0,1\end{array}$ & $\begin{array}{r}339 \\
0,2\end{array}$ & $\begin{array}{r}37136 \\
22,5\end{array}$ \\
\hline BMÖLS & $\begin{array}{l}\text { in } 1000 € \\
\text { in } \%\end{array}$ & - & - & - & - & - & - & - & - & - & - & - & - & - & - \\
\hline INSGESAMT & $\begin{array}{l}\text { in } 1000 \\
\text { in \% }\end{array}$ & $\begin{array}{r}1287326 \\
100,0\end{array}$ & $\begin{array}{r}86343 \\
6,7\end{array}$ & $\begin{array}{r}79177 \\
6,2\end{array}$ & $\begin{array}{r}194247 \\
15,1\end{array}$ & $\begin{array}{r}21365 \\
1,7\end{array}$ & $\begin{array}{r}29644 \\
2,3\end{array}$ & $\begin{array}{r}14299 \\
1,1\end{array}$ & $\begin{array}{r}291038 \\
22,6\end{array}$ & $\begin{array}{r}89881 \\
7,0\end{array}$ & $\begin{array}{r}43301 \\
3,4\end{array}$ & $\begin{array}{r}10006 \\
0,8\end{array}$ & $\begin{array}{l}336 \\
0,0\end{array}$ & $\begin{array}{r}11502 \\
0,9\end{array}$ & $\begin{array}{r}416187 \\
32,2\end{array}$ \\
\hline Stand: April 20 & & & & & & & & & & & Qu & tistik & (Bunde & stalt Statist & Österreich) \\
\hline
\end{tabular}

$\left.{ }^{1}\right)$ Erfolg.

${ }^{2}$ ) Einschließlich der im Budgetkapitel 18 veranschlagten Ausgaben für Forschung und Forschungsförderung im Bereich Umwelt. 
Aufgliederung der Jahreswerte $2001^{1}$ ) aus der Beilage T des Amtsbehelfes zum Bundesfinanzgesetz 2002 (Teil a und Teil b)

\begin{tabular}{|c|c|c|c|c|c|c|c|c|c|c|c|c|c|c|c|}
\hline \multirow{2}{*}{\multicolumn{2}{|c|}{ RESSORTS }} & \multirow[b]{2}{*}{$\begin{array}{l}\text { Ausgaben } \\
\text { des Bundes } \\
\text { für F\&E } \\
\text { insgesamt }\end{array}$} & \multicolumn{13}{|c|}{ davon für } \\
\hline & & & $\begin{array}{c}\text { Förderung } \\
\text { der Erfor- } \\
\text { schung der } \\
\text { Erde, der } \\
\text { Meere, der } \\
\text { Atmos- } \\
\text { phäre und } \\
\text { des Welt- } \\
\text { raumes }\end{array}$ & $\begin{array}{l}\text { Förderung } \\
\text { der Land- } \\
\text { und Forst- } \\
\text { wirtschaft }\end{array}$ & $\begin{array}{c}\text { Förderung } \\
\text { von Handel, } \\
\text { Gewerbe } \\
\text { und } \\
\text { Industrie }\end{array}$ & $\begin{array}{c}\text { Förderung } \\
\text { der } \\
\text { Erzeugung, } \\
\text { Speiche- } \\
\text { rung und } \\
\text { Verteilung } \\
\text { von Energie }\end{array}$ & $\begin{array}{c}\text { Förderung } \\
\text { des } \\
\text { Transport-, } \\
\text { Verkehrs- } \\
\text { und Nach- } \\
\text { richten- } \\
\text { wesens }\end{array}$ & \begin{tabular}{|c|} 
Förderung \\
des \\
Unterrichts- \\
und Bildungs \\
wesens
\end{tabular} & $\begin{array}{c}\text { Förderung } \\
\text { des } \\
\text { Gesund- } \\
\text { heits- } \\
\text { wesens }\end{array}$ & $\begin{array}{c}\text { Förderung } \\
\text { der } \\
\text { sozialen } \\
\text { und sozio- } \\
\text { ökonomi- } \\
\text { schen Ent- } \\
\text { wicklung }\end{array}$ & $\begin{array}{c}\text { Förderung } \\
\text { des } \\
\text { Umwelt- } \\
\text { schutzes }\end{array}$ & $\begin{array}{c}\text { Förderung } \\
\text { der Stadt- } \\
\text { und } \\
\text { Raum- } \\
\text { planung }\end{array}$ & $\begin{array}{c}\text { Förderung } \\
\text { der } \\
\text { Landes- } \\
\text { vertei- } \\
\text { digung }\end{array}$ & $\begin{array}{c}\text { Förderung } \\
\text { anderer } \\
\text { Zielset- } \\
\text { zungen }\end{array}$ & $\begin{array}{l}\text { Förderung } \\
\text { der all- } \\
\text { gemeinen } \\
\text { Erwei- } \\
\text { terung des } \\
\text { Wissens }\end{array}$ \\
\hline BKA & $\begin{array}{l}\text { in } 1000 € \\
\text { in } \%\end{array}$ & $\begin{array}{l}5126 \\
100,0\end{array}$ & - & - & $\begin{array}{r}6 \\
0,1\end{array}$ & $\begin{array}{l}211 \\
4,1\end{array}$ & - & - & - & $\begin{array}{r}1170 \\
22,8\end{array}$ & - & $\begin{array}{r}455 \\
8,9\end{array}$ & - & - & $\begin{array}{r}3284 \\
64,1\end{array}$ \\
\hline BMI & $\begin{array}{l}\text { in } 1000 € \\
\text { in } \%\end{array}$ & $\begin{array}{r}145 \\
100,0\end{array}$ & - & - & - & - & - & - & - & $\begin{array}{r}145 \\
100,0\end{array}$ & - & - & - & - & - \\
\hline BMBWK & $\begin{array}{l}\text { in } 1000 € \\
\text { in } \%\end{array}$ & $\begin{array}{r}975384 \\
100,0\end{array}$ & $\begin{array}{r}66945 \\
6,9\end{array}$ & $\begin{array}{r}42216 \\
4,3\end{array}$ & $\begin{array}{r}102865 \\
10,5\end{array}$ & $\begin{array}{r}14159 \\
1,5\end{array}$ & $\begin{array}{r}11742 \\
1,2\end{array}$ & $\begin{array}{r}14041 \\
1,4\end{array}$ & $\begin{array}{r}264766 \\
27,1\end{array}$ & $\begin{array}{r}75884 \\
7,8\end{array}$ & $\begin{array}{r}23950 \\
2,5\end{array}$ & $\begin{array}{r}8575 \\
0,9\end{array}$ & - & $\begin{array}{r}10730 \\
1,1\end{array}$ & $\begin{array}{r}339511 \\
34,8\end{array}$ \\
\hline BMSG & $\begin{array}{l}\text { in } 1000 € \\
\text { in } \%\end{array}$ & $\begin{array}{c}10603 \\
100,0\end{array}$ & - & $\begin{array}{r}13 \\
0,1\end{array}$ & - & - & - & - & $\begin{array}{r}6162 \\
58,2\end{array}$ & $\begin{array}{r}1898 \\
17,9\end{array}$ & $\begin{array}{r}935 \\
8,8\end{array}$ & - & - & - & $\begin{array}{r}1595 \\
15,0\end{array}$ \\
\hline BMAA & $\begin{array}{l}\text { in } 1000 € \\
\text { in } \%\end{array}$ & $\begin{array}{l}2004 \\
100,0\end{array}$ & - & - & - & $\begin{array}{r}922 \\
46,0\end{array}$ & - & - & - & $\begin{array}{r}1064 \\
53,1\end{array}$ & - & - & - & - & $\begin{array}{r}18 \\
0,9\end{array}$ \\
\hline BMJ & $\begin{array}{l}\text { in } 1000 € \\
\text { in } \%\end{array}$ & $\begin{array}{r}62 \\
100,0\end{array}$ & - & - & - & - & - & - & - & $\begin{array}{r}62 \\
100,0\end{array}$ & - & - & - & - & - \\
\hline BML & $\begin{array}{l}\text { in } 1000 € \\
\text { in } \%\end{array}$ & $\begin{array}{r}258 \\
100,0\end{array}$ & - & - & - & - & - & - & - & - & - & - & $\begin{array}{r}70 \\
27,1\end{array}$ & - & $\begin{array}{r}188 \\
72,9\end{array}$ \\
\hline BMF & $\begin{array}{l}\text { in } 1000 € \\
\text { in } \%\end{array}$ & $\begin{array}{r}163684 \\
100,0\end{array}$ & $\begin{array}{r}7647 \\
4,7\end{array}$ & $\begin{array}{r}2771 \\
1,7\end{array}$ & $\begin{array}{r}35574 \\
21,7\end{array}$ & $\begin{array}{r}3335 \\
2,0\end{array}$ & $\begin{array}{r}585 \\
0,4\end{array}$ & $\begin{array}{l}626 \\
0,4\end{array}$ & $\begin{array}{r}18660 \\
11,4\end{array}$ & $\begin{array}{r}6465 \\
3,9\end{array}$ & $\begin{array}{r}1701 \\
1,0\end{array}$ & $\begin{array}{r}469 \\
0,3\end{array}$ & - & $\begin{array}{r}37753 \\
23,1\end{array}$ & $\begin{array}{r}48098 \\
29,4\end{array}$ \\
\hline BMLFUW & $\begin{array}{l}\text { in } 1000 € \\
\text { in } \%\end{array}$ & $\begin{array}{r}45857 \\
100,0\end{array}$ & $\begin{array}{l}578 \\
1,3\end{array}$ & $\begin{array}{r}32592 \\
71,0\end{array}$ & $\begin{array}{r}1329 \\
2,9\end{array}$ & - & - & - & - & $\begin{array}{r}1552 \\
3,4\end{array}$ & $\begin{array}{r}9729 \\
21,2\end{array}$ & - & - & - & $\begin{array}{r}77 \\
0,2\end{array}$ \\
\hline BMWA & $\begin{array}{l}\text { in } 1000 € \\
\text { in } \%\end{array}$ & $\begin{array}{l}7538 \\
100,0\end{array}$ & - & - & $\begin{array}{r}5892 \\
78,1\end{array}$ & $\begin{array}{r}60 \\
0,8\end{array}$ & $\begin{array}{r}27 \\
0,4\end{array}$ & - & - & $\begin{array}{r}1276 \\
16,9\end{array}$ & - & $\begin{array}{r}60 \\
0,8\end{array}$ & - & - & $\begin{array}{r}223 \\
3,0\end{array}$ \\
\hline BMVIT & $\begin{array}{l}\text { in } 1000 € \\
\text { in } \%\end{array}$ & $\begin{array}{r}190067 \\
100,0\end{array}$ & $\begin{array}{r}13174 \\
6,9\end{array}$ & $\begin{array}{r}1417 \\
0,7\end{array}$ & $\begin{array}{r}76558 \\
40,4\end{array}$ & $\begin{array}{r}4283 \\
2,3\end{array}$ & $\begin{array}{r}22680 \\
11,9\end{array}$ & $\begin{array}{r}270 \\
0,1\end{array}$ & $\begin{array}{r}19065 \\
10,0\end{array}$ & $\begin{array}{r}1492 \\
0,8\end{array}$ & $\begin{array}{r}7958 \\
4,2\end{array}$ & $\begin{array}{r}581 \\
0,3\end{array}$ & $\begin{array}{r}270 \\
0,1\end{array}$ & $\begin{array}{l}372 \\
0,2\end{array}$ & $\begin{array}{r}41947 \\
22,1\end{array}$ \\
\hline BMÖLS & $\begin{array}{l}\text { in } 1000 € \\
\text { in } \%\end{array}$ & - & - & $\begin{array}{l}- \\
-\end{array}$ & - & $\begin{array}{l}- \\
-\end{array}$ & - & $\begin{array}{l}- \\
-\end{array}$ & - & - & - & - & - & $\begin{array}{l}- \\
-\end{array}$ & $\begin{array}{l}- \\
-\end{array}$ \\
\hline INSGESAMT & in 1000 & 1400728 & 88344 & 79009 & 222224 & 22970 & 35034 & 14937 & 308653 & 91008 & 44273 & 10140 & 340 & 48855 & 434941 \\
\hline & in \% & 100,0 & 6,3 & 5,6 & 15,9 & 1,6 & 2,5 & 1,1 & 22,0 & 6,5 & 3,2 & 0,7 & 0,0 & 3,5 & 31,1 \\
\hline
\end{tabular}

1) Bundesvoranschlag.

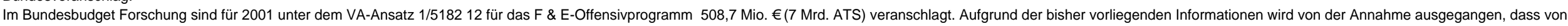

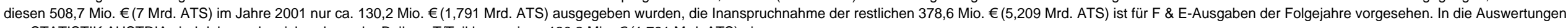
von STATISTIK AUSTRIA sind daher - abweichend von der Beilage T/Teil b - nur jene 130,2 Mio. $€$ (1,791 Mrd. ATS) eingegangen. 


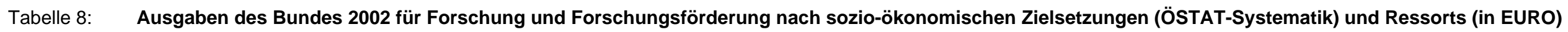
Aufgliederung der Jahreswerte $2002{ }^{1}$ ) aus der Beilage T des Amtsbehelfes zum Bundesfinanzgesetz 2002 (Teil a und Teil b)

\begin{tabular}{|c|c|c|c|c|c|c|c|c|c|c|c|c|c|c|c|}
\hline \multirow{2}{*}{\multicolumn{2}{|c|}{ RESSORTS }} & \multirow[b]{2}{*}{$\begin{array}{l}\text { Ausgaben } \\
\text { des Bundes } \\
\text { für F\&E } \\
\text { insgesamt }\end{array}$} & \multicolumn{13}{|c|}{ davon für } \\
\hline & & & $\begin{array}{c}\text { Förderung der } \\
\text { Erfor- } \\
\text { schung der } \\
\text { Erde, der } \\
\text { Meere, der } \\
\text { Atmos- } \\
\text { phäre und } \\
\text { des Welt- } \\
\text { raumes }\end{array}$ & $\begin{array}{l}\text { Förderung } \\
\text { der Land- } \\
\text { und Forst- } \\
\text { wirtschaft }\end{array}$ & $\begin{array}{c}\text { Förderung } \\
\text { von Handel, } \\
\text { Gewerbe } \\
\text { und } \\
\text { Industrie }\end{array}$ & \begin{tabular}{|c|} 
Förderung der \\
Erzeugung, \\
Speiche- \\
rung und \\
Verteilung \\
von Energie
\end{tabular} & \begin{tabular}{c|} 
Förderung \\
des Transport-, \\
Verkehrs- \\
und Nach- \\
richten- \\
wesens
\end{tabular} & \begin{tabular}{|c|} 
Förderung \\
des \\
Unterrichts- \\
und Bildungs- \\
wesens
\end{tabular} & $\begin{array}{l}\text { Förderung } \\
\text { des } \\
\text { Gesund- } \\
\text { heits- } \\
\text { wesens }\end{array}$ & $\begin{array}{c}\text { Förderung der } \\
\text { sozialen } \\
\text { und sozio- } \\
\text { ökonomi- } \\
\text { schen Ent- } \\
\text { wicklung }\end{array}$ & $\begin{array}{l}\text { Förderung } \\
\text { des } \\
\text { Umwelt- } \\
\text { schutzes }\end{array}$ & \begin{tabular}{|} 
Förderung der \\
Stadt- \\
und \\
Raum- \\
planung
\end{tabular} & $\begin{array}{l}\text { Förderung } \\
\text { der } \\
\text { Landes- } \\
\text { vertei- } \\
\text { digung }\end{array}$ & $\begin{array}{l}\text { Förderung } \\
\text { anderer } \\
\text { Zielset- } \\
\text { zungen }\end{array}$ & $\begin{array}{l}\text { Förderung } \\
\text { der all- } \\
\text { gemeinen } \\
\text { Erwei- } \\
\text { terung des } \\
\text { Wissens }\end{array}$ \\
\hline BKA & $\begin{array}{l}\text { in } 1000 € \\
\text { in } \%\end{array}$ & $\begin{array}{l}4880 \\
100,0\end{array}$ & - & - & $\begin{array}{r}6 \\
0,1\end{array}$ & - & - & - & - & $\begin{array}{r}1140 \\
23,4\end{array}$ & - & $\begin{array}{r}442 \\
9,1\end{array}$ & - & - & $\begin{array}{r}3292 \\
67,4\end{array}$ \\
\hline BMI & $\begin{array}{l}\text { in } 1000 € \\
\text { in } \%\end{array}$ & $\begin{array}{r}91 \\
100,0\end{array}$ & - & - & - & - & - & - & - & $\begin{array}{r}91 \\
100,0\end{array}$ & - & - & - & - & - \\
\hline BMBWK & $\begin{array}{l}\text { in } 1000 € \\
\text { in } \%\end{array}$ & $\begin{array}{r}974117 \\
100,0\end{array}$ & $\begin{array}{r}67424 \\
6,9\end{array}$ & $\begin{array}{r}42280 \\
4,3\end{array}$ & $\begin{array}{r}106132 \\
10,9\end{array}$ & $\begin{array}{r}14094 \\
1,4\end{array}$ & $\begin{array}{r}11697 \\
1,2\end{array}$ & $\begin{array}{r}13898 \\
1,4\end{array}$ & $\begin{array}{r}260362 \\
26,7\end{array}$ & $\begin{array}{r}74901 \\
7,7\end{array}$ & $\begin{array}{r}23903 \\
2,5\end{array}$ & $\begin{array}{r}8551 \\
0,9\end{array}$ & - & $\begin{array}{r}10713 \\
1,1\end{array}$ & $\begin{array}{r}340162 \\
35,0\end{array}$ \\
\hline BMSG & $\begin{array}{l}\text { in } 1000 € \\
\text { in } \%\end{array}$ & $\begin{array}{r}10685 \\
100,0\end{array}$ & - & $\begin{array}{l}13 \\
0,1\end{array}$ & - & - & - & - & $\begin{array}{r}6168 \\
57,7\end{array}$ & $\begin{array}{r}1865 \\
17,5\end{array}$ & $\begin{array}{r}957 \\
9,0\end{array}$ & - & - & - & $\begin{array}{r}1682 \\
15,7\end{array}$ \\
\hline BMAA & $\begin{array}{l}\text { in } 1000 € \\
\text { in } \%\end{array}$ & $\begin{array}{l}1993 \\
100,0\end{array}$ & - & - & - & $\begin{array}{l}923 \\
46,3\end{array}$ & - & - & - & $\begin{array}{r}1052 \\
52,8\end{array}$ & - & - & - & - & $\begin{array}{l}18 \\
0,9\end{array}$ \\
\hline BMJ & $\begin{array}{l}\text { in } 1000 € \\
\text { in } \%\end{array}$ & $\begin{array}{r}73 \\
100,0\end{array}$ & - & - & - & - & - & - & - & $\begin{array}{r}73 \\
100,0\end{array}$ & - & - & - & - & - \\
\hline BML & $\begin{array}{l}\text { in } 1000 € \\
\text { in } \%\end{array}$ & $\begin{array}{r}267 \\
100,0\end{array}$ & - & - & - & - & - & - & - & - & - & - & $\begin{array}{r}79 \\
29,6\end{array}$ & - & $\begin{array}{l}188 \\
70,4\end{array}$ \\
\hline BMF & $\begin{array}{l}\text { in } 1000 € \\
\text { in } \%\end{array}$ & $\begin{array}{r}49937 \\
100,0\end{array}$ & $\begin{array}{r}3537 \\
7,1\end{array}$ & $\begin{array}{r}2476 \\
5,0\end{array}$ & $\begin{array}{r}6180 \\
12,4\end{array}$ & $\begin{array}{r}996 \\
2,0\end{array}$ & $\begin{array}{r}817 \\
1,6\end{array}$ & $\begin{array}{r}799 \\
1,6\end{array}$ & $\begin{array}{r}9857 \\
19,7\end{array}$ & $\begin{array}{r}6310 \\
12,6\end{array}$ & $\begin{array}{r}1391 \\
2,8\end{array}$ & $\begin{array}{c}491 \\
1,0\end{array}$ & - & $\begin{array}{r}637 \\
1,3\end{array}$ & $\begin{array}{r}16446 \\
32,9\end{array}$ \\
\hline BMLFUW & $\begin{array}{l}\text { in } 1000 € \\
\text { in } \%\end{array}$ & $\begin{array}{r}44080 \\
100,0\end{array}$ & $\begin{array}{c}581 \\
1,3\end{array}$ & $\begin{array}{r}30199 \\
68,5\end{array}$ & $\begin{array}{r}1329 \\
3,0\end{array}$ & - & - & - & $\begin{array}{l}- \\
-\end{array}$ & $\begin{array}{r}1552 \\
3,5\end{array}$ & $\begin{array}{r}10341 \\
23,5\end{array}$ & - & - & - & $\begin{array}{l}78 \\
0,2\end{array}$ \\
\hline BMWA & $\begin{array}{l}\text { in } 1000 € \\
\text { in } \%\end{array}$ & $\begin{array}{l}7799 \\
100,0\end{array}$ & - & - & $\begin{array}{r}6170 \\
79,1\end{array}$ & $\begin{array}{l}60 \\
0,8\end{array}$ & $\begin{array}{l}27 \\
0,3\end{array}$ & - & - & $\begin{array}{r}1259 \\
16,1\end{array}$ & - & $\begin{array}{l}60 \\
0,8\end{array}$ & - & - & $\begin{array}{r}223 \\
2,9\end{array}$ \\
\hline BMVIT & $\begin{array}{l}\text { in } 1000 € \\
\text { in } \%\end{array}$ & $\begin{array}{r}189011 \\
100,0\end{array}$ & $\begin{array}{r}13633 \\
7,2\end{array}$ & $\begin{array}{r}1442 \\
0,8\end{array}$ & $\begin{array}{r}75632 \\
40,0\end{array}$ & $\begin{array}{r}4254 \\
2,3\end{array}$ & $\begin{array}{r}22212 \\
11,8\end{array}$ & $\begin{array}{r}278 \\
0,1\end{array}$ & $\begin{array}{r}19123 \\
10,1\end{array}$ & $\begin{array}{r}1508 \\
0,8\end{array}$ & $\begin{array}{r}8052 \\
4,3\end{array}$ & $\begin{array}{c}597 \\
0,3\end{array}$ & $\begin{array}{r}278 \\
0,1\end{array}$ & $\begin{array}{r}372 \\
0,2\end{array}$ & $\begin{array}{r}41630 \\
22,0\end{array}$ \\
\hline BMÖLS & $\begin{array}{l}\text { in } 1000 € \\
\text { in } \%\end{array}$ & - & - & - & - & - & - & - & - & - & - & - & - & - & - \\
\hline INSGESAMT & $\begin{array}{l}\text { in } 1000 \\
\text { in } \%\end{array}$ & $\begin{array}{r}1282933 \\
100,0\end{array}$ & $\begin{array}{r}85175 \\
6.6\end{array}$ & $\begin{array}{r}76410 \\
6.0\end{array}$ & $\begin{array}{r}195449 \\
15,2\end{array}$ & 20327 & $\begin{array}{r}34753 \\
2.7\end{array}$ & $\begin{array}{r}14975 \\
1.2\end{array}$ & $\begin{array}{r}295510 \\
23,0\end{array}$ & $\begin{array}{r}89751 \\
7.0\end{array}$ & $\begin{array}{r}44644 \\
3.5\end{array}$ & $\begin{array}{r}10141 \\
0.8\end{array}$ & $\begin{array}{r}357 \\
0.0\end{array}$ & $\begin{array}{r}11722 \\
0,9\end{array}$ & $\begin{array}{r}403719 \\
31.5\end{array}$ \\
\hline
\end{tabular}



Sektoren/ Bereichen) und vergebenden Ressorts (in ATS)

Auswertung der Faktendokumentation der Bundesdienststellen für 2000 (Stand: Juli 2001) einschließ lich "große" Globalförderungen ${ }^{1}$ )

\begin{tabular}{|c|c|c|c|c|c|c|c|c|c|c|c|c|c|c|c|c|c|c|c|c|c|c|c|c|c|c|}
\hline \multirow{4}{*}{ RESSORTS } & \multirow[b]{3}{*}{$\begin{array}{l}\text { Teilbeträge } \\
2000\end{array}$} & \multicolumn{25}{|c|}{ davon vergeben an } \\
\hline & & \multicolumn{5}{|c|}{ HOCHSCHULSEKTOR } & \multicolumn{8}{|c|}{ SEKTOR STAAT } & \multicolumn{3}{|c|}{$\begin{array}{c}\text { PRIVATER } \\
\text { GEMEINNÜTZIGER } \\
\text { SEKTOR }\end{array}$} & \multicolumn{6}{|c|}{ UNTERNEHMENSSEKTOR } & \multirow[b]{2}{*}{ 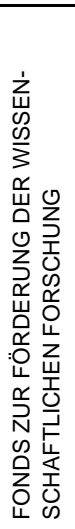 } & \multirow[b]{2}{*}{ 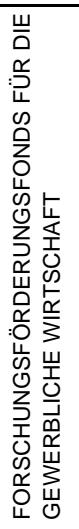 } & \multirow[b]{2}{*}{ 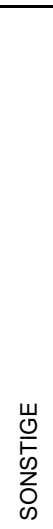 } \\
\hline & & 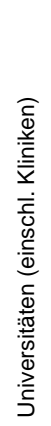 & 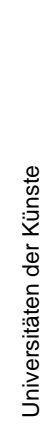 & 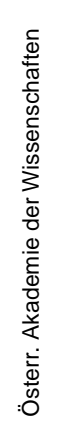 & 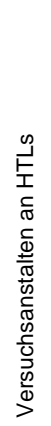 & 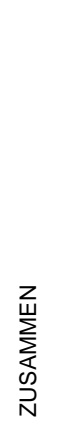 & 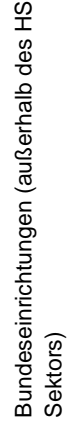 & 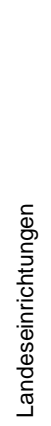 & 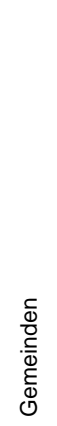 & 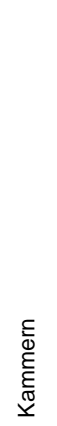 & 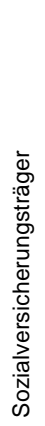 & 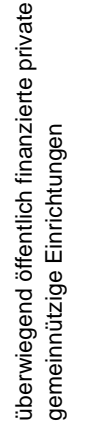 & 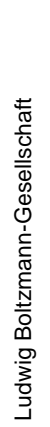 & 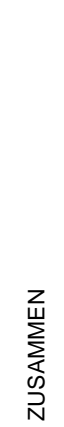 & 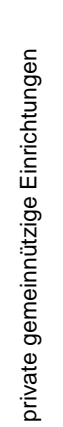 & 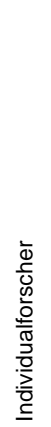 & 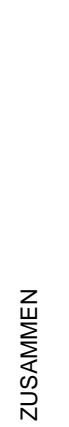 & 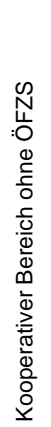 & 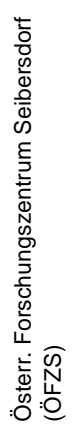 & 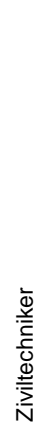 & 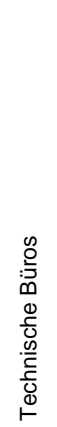 & 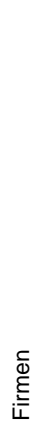 & 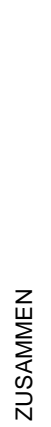 & & & \\
\hline & in Schilling & & & & & & & & & & & & in & & & & & & & & & & & & & \\
\hline
\end{tabular}

\section{BKA}

BMI

BMBWK

BMVIT

BMSG

BMAA

BMJ

BML

BMF

BMLFUW

BMWA

INSGESAMT
1197548208

$2064405863 \quad 1,4$

$11148269 \quad 12,7$

$\begin{array}{cc}- & - \\ 0,0 & 42,2\end{array}$

$0,0 \quad 49,2$

$0,7 \quad 0,1$

$\begin{array}{lll}1,4 & 0,0 & 0,0\end{array}$

850000

$5929830 \quad 43,7$

- 12,5

-

- 56,2

$\begin{array}{llllllll}58837324 & 41,1 & - & 3,5 & - & 44,6 & 17,8 & 0,1\end{array}$

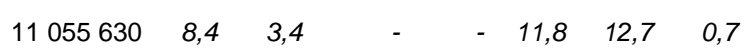

$\begin{array}{llllllll}3350166900 & 4,3 & 0,0 & 15,1 & 0,0 & 19,4 & 0,6 & 0,1\end{array}$

Stand: April 2002

$$
\begin{array}{rrrrrrrrrrrrrrrrr}
100,0 & - & 100,0 & - & - & - & - & - & - & - & - & - & - & - & - \\
16,2 & 4,4 & 21,4 & 4,0 & 0,5 & 4,5 & 0,7 & 7,5 & 0,1 & 0,2 & 0,3 & 8,8 & 15,9 & - & 0,2 \\
11,8 & 0,1 & 11,9 & 1,2 & 0,2 & 1,4 & 2,7 & 19,1 & 0,1 & 0,1 & 0,8 & 22,8 & 25,7 & 36,7 & 0,1 \\
9,3 & 1,3 & 10,6 & 50,8 & 0,4 & 51,2 & - & 25,5 & - & - & - & 25,5 & - & - & - \\
- & - & - & - & - & - & - & - & - & - & - & - & - & - & - \\
100,0 & - & 100,0 & - & - & - & - & - & - & - & - & - & - & - & - \\
12,7 & - & 12,7 & - & 7,1 & 7,1 & 9,9 & 9,1 & - & - & 5,0 & 24,0 & - & - & - \\
- & - & - & - & - & - & - & - & - & - & - & - & - & - & - \\
8,8 & 0,9 & 27,6 & 6,3 & 1,0 & 7,3 & 1,3 & 9,4 & 0,9 & 2,2 & 2,8 & 16,6 & - & - & 3,9 \\
14,1 & - & 28,0 & 1,8 & - & 1,8 & 32,9 & - & - & 0,9 & 24,6 & 58,4 & - & - & - \\
13,4 & \mathbf{1 , 7} & \mathbf{1 5 , 8} & \mathbf{2 , 5} & \mathbf{0 , 4} & \mathbf{2 , 9} & \mathbf{2 , 0} & \mathbf{1 4 , 7} & \mathbf{0 , 1} & \mathbf{0 , 2} & \mathbf{0 , 8} & \mathbf{1 7 , 8} & \mathbf{2 1 , 5} & \mathbf{2 2 , 4} & \mathbf{0 , 2}
\end{array}
$$

Quelle: Statistik Austria (Bundesanstalt Statistik Österreich)

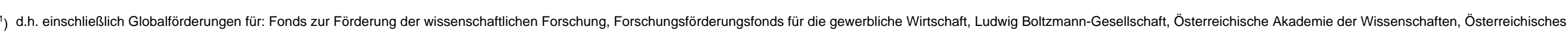
Forschungszentrum Seibersdorf (insgesamt 2562100673 Schilling). Die in der Faktendokumentation 2000 nicht ausgewiesenen Förderungsmittel seitens des BMBWK an den FWF sowie seitens des BMVIT an den FFF wurden aus dem
Bundesrechnungsabschluss 2000 ermittelt und entsprechend berücksichtigt. 
Sektoren/ Bereichen) und vergebenden Ressorts (in ATS)

Auswertung der Faktendokumentation der Bundesdienststellen für 2000 (Stand: Juli 2001) ohne "große" Globalförderungen ${ }^{1}$ )

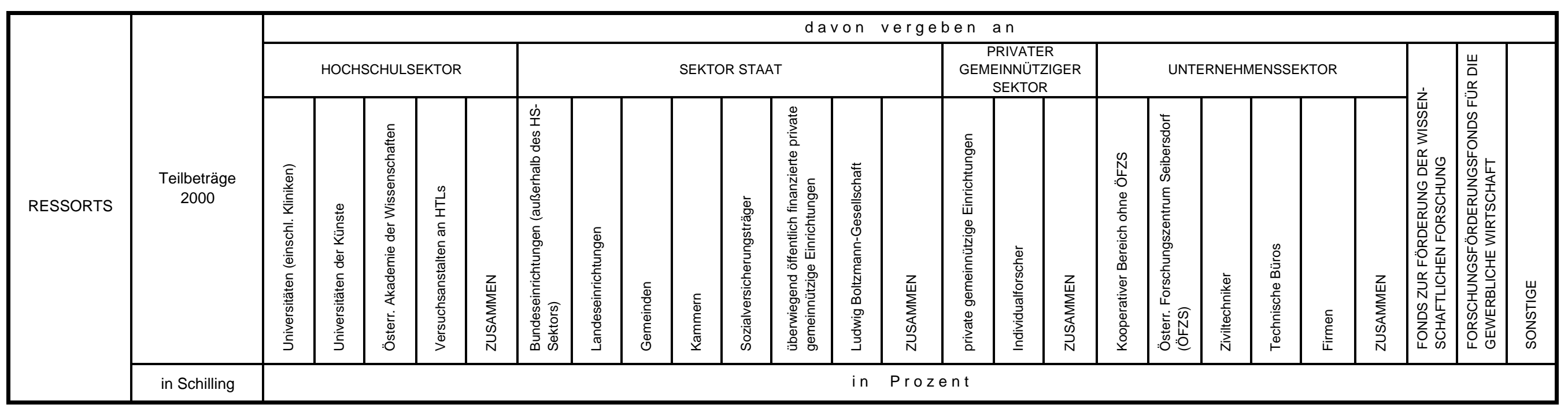

\section{BKA}

BMI

BMBWK

BMVIT

BMSG

BMAA

BMJ

BML

BMF

BMLFUW

BMWA

INSGESAMT
391776

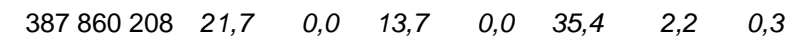

$\begin{array}{llllllll}311993190 & 9,5 & - & 0,1 & - & 9,6 & 0,3 & 0,2\end{array}$

850000

$5929830 \quad 43,7$

$58837324 \quad 41,1$

$11055630 \quad 8,4 \quad 3,4$

$788066227 \quad 18,2$

0,1
$-\quad-12,7$

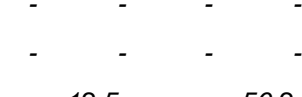

$$
\text { - } \quad \text { - } \quad \text { - }
$$

$11148269 \quad 12,7$

Stand: April 2002

$$
\begin{array}{rrrrrrrrrrrrrrrrr}
100,0 & - & 100,0 & - & - & - & - & - & - & - & - & - & - & - & - \\
41,6 & 0,8 & 44,9 & 12,4 & 1,7 & 14,1 & 2,0 & 0,9 & 0,3 & 0,7 & 1,0 & 4,9 & - & - & 0,7 \\
31,2 & 0,9 & 32,6 & 8,2 & 1,3 & 9,5 & 17,3 & 15,3 & 0,6 & 0,5 & 5,5 & 39,2 & 3,5 & 5,1 & 0,5 \\
9,3 & 1,3 & 10,6 & 50,8 & 0,4 & 51,2 & - & 25,5 & - & - & - & 25,5 & - & - & - \\
- & - & - & - & - & - & - & - & - & - & - & - & - & - & - \\
100,0 & - & 100,0 & - & - & - & - & - & - & - & - & - & - & - & - \\
12,7 & - & 12,7 & - & 7,1 & 7,1 & 9,9 & 9,1 & - & - & 5,0 & 24,0 & - & - & - \\
- & - & - & - & - & - & - & - & - & - & - & - & - & - & - \\
8,8 & 0,9 & 27,6 & 6,3 & 1,0 & 7,3 & 1,3 & 9,4 & 0,9 & 2,2 & 2,8 & 16,6 & - & - & 3,9 \\
14,1 & - & 28,0 & 1,8 & - & 1,8 & 32,9 & - & - & 0,9 & 24,6 & 58,4 & - & - & - \\
\mathbf{3 4 , 1} & \mathbf{0 , 8} & \mathbf{3 7 , 8} & \mathbf{1 0 , 6} & \mathbf{1 , 5} & \mathbf{1 2 , 1} & \mathbf{8 , 5} & \mathbf{7 , 6} & \mathbf{0 , 4} & \mathbf{0 , 7} & \mathbf{3 , 3} & \mathbf{2 0 , 5} & \mathbf{1 , 4} & \mathbf{2 , 0} & \mathbf{0 , 8}
\end{array}
$$

Quelle: Statistik Austria (Bundesanstalt Statistik Österreich)

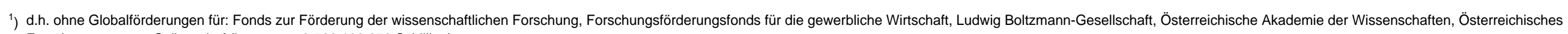
Forschungszentrum Seibersdorf (insgesamt 2562100673 Schilling). 
Tabelle 11: Forschungsförderungen und Forschungsaufträge des Bundes 2000 nach sozio-ökonomischen Zielsetzungen und vergebenden Ressorts (in ATS)

Auswertung der Faktendokumentation der Bundesdienststellen für 2000 (Stand: Juli 2001) nach sozio-ökonomischen Zielsetzungen (ÖSTAT-Klassifikation),einschließ lich"große" Globalförderungen ${ }^{\text {) }}$

\begin{tabular}{|c|c|c|c|c|c|c|c|c|c|c|c|c|c|c|c|}
\hline \multirow{2}{*}{\multicolumn{2}{|c|}{ RESSORTS }} & \multirow[b]{2}{*}{$\begin{array}{l}\text { Teilbeträge } \\
2000\end{array}$} & \multicolumn{13}{|c|}{ davon für } \\
\hline & & & $\begin{array}{c}\text { Förderung der } \\
\text { Erforschung } \\
\text { der Erde, } \\
\text { der Meere, } \\
\text { der Atmosphäre } \\
\text { und des } \\
\text { Weltraumes }\end{array}$ & $\begin{array}{l}\text { Förderung der } \\
\text { Land- und } \\
\text { Forstwirtschaft }\end{array}$ & $\begin{array}{c}\text { Förderung von } \\
\text { Handel, } \\
\text { Gewerbe und } \\
\text { Industrie }\end{array}$ & $\begin{array}{l}\text { Förderung der } \\
\text { Erzeugung, } \\
\text { Speicherung } \\
\text { und Verteilung } \\
\text { von Energie }\end{array}$ & $\begin{array}{l}\text { Förderung des } \\
\text { Transport-, } \\
\text { Verkehrs- und } \\
\text { Nachrichten- } \\
\text { wesens }\end{array}$ & $\begin{array}{c}\text { Förderung des } \\
\text { Unterrichts- } \\
\text { und Bildungs- } \\
\text { wesens }\end{array}$ & $\begin{array}{c}\text { Förderung des } \\
\text { Gesund- } \\
\text { hitswesesens }\end{array}$ & $\begin{array}{l}\text { Förderung der } \\
\text { sozialen und } \\
\text { sozio-ökonomi- } \\
\text { schen Ent- } \\
\text { wicklung }\end{array}$ & $\begin{array}{l}\text { Förderung des } \\
\text { Umwelt- } \\
\text { schutzes }\end{array}$ & $\begin{array}{l}\text { Förderung der } \\
\text { Stadt- und } \\
\text { Raum- } \\
\text { planung }\end{array}$ & $\begin{array}{l}\text { Förderung der } \\
\text { Landesverteidi- } \\
\quad \text { gung }\end{array}$ & $\begin{array}{l}\text { Förderung } \\
\text { anderer Ziel- } \\
\text { setzungen }\end{array}$ & $\begin{array}{l}\text { Förderung der } \\
\text { allgemeinen } \\
\text { Erweiterung } \\
\text { des Wissens }\end{array}$ \\
\hline BKA & $\begin{array}{l}\text { in Schilling } \\
\text { in \% }\end{array}$ & - & - & - & - & - & - & - & - & - & - & - & - & - & \\
\hline BMI & $\begin{array}{l}\text { in Schilling } \\
\text { in \% }\end{array}$ & $\begin{array}{r}391776 \\
100,0\end{array}$ & - & - & - & - & $\begin{array}{r}391776 \\
100,0\end{array}$ & - & - & - & - & - & - & - & \\
\hline BMBWK & $\begin{array}{l}\text { in Schilling } \\
\text { in \% }\end{array}$ & $\begin{array}{r}1197548208 \\
100,0\end{array}$ & $\begin{array}{r}87286580 \\
7,3\end{array}$ & $\begin{array}{r}11782176 \\
1,0\end{array}$ & 87262166 & $\begin{array}{r}13035802 \\
1,1\end{array}$ & $\begin{array}{r}18619424 \\
1,6\end{array}$ & $\begin{array}{r}7976403 \\
0,7\end{array}$ & $\begin{array}{r}167347510 \\
13,9\end{array}$ & $\begin{array}{r}145026811 \\
12,1\end{array}$ & $\begin{array}{r}44541816 \\
3,7\end{array}$ & $\begin{array}{r}12916981 \\
1,1\end{array}$ & $\begin{array}{r}864810 \\
0,1\end{array}$ & $\begin{array}{r}9119500 \\
0,8\end{array}$ & $\begin{array}{r}591768229 \\
49,3\end{array}$ \\
\hline BMVIT & $\begin{array}{l}\text { in Schilling } \\
\text { in \% }\end{array}$ & $\begin{array}{r}2064405863 \\
100,0\end{array}$ & $\begin{array}{r}48048140 \\
2,3\end{array}$ & $\begin{array}{r}21345742 \\
1,0\end{array}$ & $\begin{array}{r}826171893 \\
40,0\end{array}$ & $\begin{array}{r}100894552 \\
4,9\end{array}$ & $\begin{array}{r}85231301 \\
4,1\end{array}$ & $\begin{array}{r}19867340 \\
1,0\end{array}$ & $\begin{array}{r}287172123 \\
13,9\end{array}$ & $\begin{array}{r}50981293 \\
2,5\end{array}$ & $\begin{array}{r}144791065 \\
7,0\end{array}$ & $\begin{array}{r}8396072 \\
0,4\end{array}$ & $\begin{array}{r}3456940 \\
0,2\end{array}$ & $\begin{array}{r}12257853 \\
0,6\end{array}$ & $\begin{array}{r}455791549 \\
22,1\end{array}$ \\
\hline BMSG & $\begin{array}{l}\text { in Schilling } \\
\text { in \% }\end{array}$ & $\begin{array}{r}11148269 \\
100,0\end{array}$ & - & $\begin{array}{r}300000 \\
2,7\end{array}$ & - & - & - & - & $\begin{array}{r}1006585 \\
9,0\end{array}$ & $\begin{array}{r}6509498 \\
58,4\end{array}$ & $\begin{array}{r}959079 \\
8,6\end{array}$ & - & - & - & $\begin{array}{r}2373107 \\
21,3\end{array}$ \\
\hline BMAA & $\begin{array}{l}\text { in Schilling } \\
\text { in \% }\end{array}$ & & - & - & - & - & - & - & - & & - & - & - & - & \\
\hline BMJ & $\begin{array}{l}\text { in Schilling } \\
\text { in \% }\end{array}$ & $\begin{array}{r}850000 \\
100,0\end{array}$ & - & - & - & - & - & - & - & $\begin{array}{r}850000 \\
100,0\end{array}$ & - & - & - & - & - \\
\hline BML & $\begin{array}{l}\text { in Schilling } \\
\text { in \% }\end{array}$ & $\begin{array}{r}5929830 \\
100,0\end{array}$ & - & - & - & - & - & - & $\begin{array}{r}1243330 \\
21,0\end{array}$ & $\begin{array}{r}818000 \\
13,8\end{array}$ & $\begin{array}{r}192500 \\
3,2\end{array}$ & - & $\begin{array}{r}2548000 \\
43,0\end{array}$ & - & $\begin{array}{r}1128000 \\
19,0\end{array}$ \\
\hline BMF & $\begin{array}{l}\text { in Schilling } \\
\text { in \% }\end{array}$ & - & - & - & - & - & - & - & - & - & - & - & - & - & - \\
\hline BMLFUW & $\begin{array}{l}\text { in Schilling } \\
\text { in \% }\end{array}$ & $\begin{array}{r}58837324 \\
100,0\end{array}$ & $\begin{array}{r}4275533 \\
7,3\end{array}$ & $\begin{array}{r}19862142 \\
33,8\end{array}$ & $\begin{array}{r}4360290 \\
7,4\end{array}$ & $\begin{array}{r}1209000 \\
2,1\end{array}$ & - & - & $\begin{array}{r}2612003 \\
4,4\end{array}$ & $\begin{array}{r}3167705 \\
5,4\end{array}$ & $\begin{array}{r}14302618 \\
24,3\end{array}$ & $\begin{array}{r}437500 \\
0,7\end{array}$ & - & - & $\begin{array}{r}8610533 \\
14,6\end{array}$ \\
\hline BMWA & $\begin{array}{l}\text { in Schilling } \\
\text { in \% }\end{array}$ & $\begin{array}{r}11055630 \\
100,0\end{array}$ & $\begin{array}{r}2701000 \\
24,4\end{array}$ & $\begin{array}{r}367000 \\
3,3\end{array}$ & $\begin{array}{r}2917600 \\
26,4\end{array}$ & - & $\begin{array}{r}57270 \\
0,5\end{array}$ & - & $\begin{array}{r}1120000 \\
10,1\end{array}$ & $\begin{array}{r}1145000 \\
10,4\end{array}$ & $\begin{array}{r}1500000 \\
13,6\end{array}$ & - & - & - & $\begin{array}{r}1247760 \\
11,3\end{array}$ \\
\hline INSGESAM? & $\begin{array}{l}\text { in Schilling } \\
\text { in \% }\end{array}$ & $\begin{array}{r}3350166900 \\
100,0\end{array}$ & $\begin{array}{r}142311253 \\
4,2\end{array}$ & $\begin{array}{r}53657060 \\
1,6\end{array}$ & $\begin{array}{r}920711949 \\
27,6\end{array}$ & $\begin{array}{r}115139354 \\
3,4\end{array}$ & $\begin{array}{r}104299771 \\
3,1\end{array}$ & $\begin{array}{r}27843743 \\
0,8\end{array}$ & $\begin{array}{r}460501551 \\
13,7\end{array}$ & $\begin{array}{r}208498307 \\
6,2\end{array}$ & $\begin{array}{r}206287078 \\
6,2\end{array}$ & $\begin{array}{r}21750553 \\
0,6\end{array}$ & $\begin{array}{r}6869750 \\
0,2\end{array}$ & $\begin{array}{r}21377353 \\
0,6\end{array}$ & $\begin{array}{r}1060919178 \\
31,8\end{array}$ \\
\hline
\end{tabular}




\section{Tabelle 12: Forschungsförderungen und Forschungsaufträge des Bundes 2000 nach sozio-ökonomischen Zielsetzungen und vergebenden Ressorts (in ATS)}

Auswertung der Faktendokumentation der Bundesdienststellen für 2000 (Stand: Juli 2001) nach sozio-ökonomischen Zielsetzungen (ÖSTAT-Klassifikation), ohne "große" Globalförderungen ${ }^{1}$ )

\begin{tabular}{|c|c|c|c|c|c|c|c|c|c|c|c|c|c|c|c|}
\hline \multirow{2}{*}{\multicolumn{2}{|c|}{ RESSORTS }} & \multirow[b]{2}{*}{$\begin{array}{l}\text { Teilbeträge } \\
2000\end{array}$} & \multicolumn{13}{|c|}{ davon für } \\
\hline & & & \begin{tabular}{|c} 
Förderung der \\
Erforschung \\
der Erde, \\
der Meere, \\
der Atmosphäre \\
und des \\
Weltraumes
\end{tabular} & $\begin{array}{l}\text { Förderung der } \\
\text { Land- und } \\
\text { Forstwirtschaft }\end{array}$ & $\begin{array}{c}\text { Förderung von } \\
\text { Handel, } \\
\text { Gewerbe und } \\
\text { Industrie }\end{array}$ & $\begin{array}{c}\text { Förderung der } \\
\text { Erzeugung, } \\
\text { Speicherung } \\
\text { und Verteilung } \\
\text { von Energie }\end{array}$ & $\begin{array}{l}\text { Förderung des } \\
\text { Transport-, } \\
\text { Verkehrs- und } \\
\text { Nachrichten- } \\
\text { wesens }\end{array}$ & $\begin{array}{l}\text { Förderung des } \\
\text { Unterrichts- } \\
\text { und Bildungs- } \\
\text { wesens }\end{array}$ & $\begin{array}{l}\text { Förderung des } \\
\text { Gesund- } \\
\text { heitswesens }\end{array}$ & $\begin{array}{l}\text { Förderung der } \\
\text { sozialen und } \\
\text { sozio-ökonomi- } \\
\text { schen Ent- } \\
\text { wicklung }\end{array}$ & $\begin{array}{l}\text { Förderung des } \\
\text { Umwelt- } \\
\text { schutzes }\end{array}$ & $\begin{array}{l}\text { Förderung der } \\
\text { Stadt- und } \\
\text { Raum- } \\
\text { planung }\end{array}$ & $\begin{array}{c}\text { Förderung der } \\
\text { Landesverteidi- } \\
\text { gung }\end{array}$ & $\begin{array}{l}\text { Förderung } \\
\text { anderer Ziel- } \\
\text { setzungen }\end{array}$ & $\begin{array}{l}\text { Förderung der } \\
\text { allgemeinen } \\
\text { Erweiterung } \\
\text { des Wissens }\end{array}$ \\
\hline BKA & $\begin{array}{l}\text { in Schilling } \\
\text { in \% }\end{array}$ & - & - & - & - & - & - & - & - & - & - & - & - & - & . \\
\hline BMI & $\begin{array}{l}\text { in Schilling } \\
\text { in \% }\end{array}$ & $\begin{array}{r}391776 \\
100,0\end{array}$ & - & - & - & - & $\begin{array}{r}391776 \\
100,0\end{array}$ & - & . & - & - & 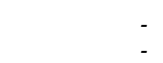 & - & - & . \\
\hline BMBWK & $\begin{array}{l}\text { in Schilling } \\
\text { in \% }\end{array}$ & $\begin{array}{r}387860208 \\
100,0\end{array}$ & $\begin{array}{r}20403645 \\
5,3\end{array}$ & $\begin{array}{r}6841950 \\
1,8\end{array}$ & $\begin{array}{r}8714499 \\
2,2\end{array}$ & $\begin{array}{r}3452302 \\
0,9\end{array}$ & $\begin{array}{r}5664374 \\
1,5\end{array}$ & $\begin{array}{r}6766591 \\
1,7\end{array}$ & $\begin{array}{r}30876433 \\
8,0\end{array}$ & $\begin{array}{r}96483035 \\
24,9\end{array}$ & $\begin{array}{r}18661645 \\
4,8\end{array}$ & $\begin{array}{r}2438862 \\
0,6\end{array}$ & - & $\begin{array}{r}7405000 \\
1,9\end{array}$ & $\begin{array}{r}180151872 \\
46,4\end{array}$ \\
\hline BMVIT & $\begin{array}{l}\text { in Schilling } \\
\text { in \% }\end{array}$ & $\begin{array}{r}311993190 \\
100,0\end{array}$ & $\begin{array}{r}18096700 \\
5,8\end{array}$ & $\begin{array}{r}328936 \\
0,1\end{array}$ & $\begin{array}{r}55987470 \\
17,9\end{array}$ & $\begin{array}{r}12232751 \\
3,9\end{array}$ & $\begin{array}{r}10608403 \\
3,4\end{array}$ & $\begin{array}{r}16410400 \\
5,3\end{array}$ & $\begin{array}{r}16596361 \\
5,3\end{array}$ & $\begin{array}{r}32118913 \\
10,3\end{array}$ & $\begin{array}{r}36458662 \\
11,7\end{array}$ & $\begin{array}{r}962692 \\
0,3\end{array}$ & - & $\begin{array}{r}7582353 \\
2,4\end{array}$ & $\begin{array}{r}104609549 \\
33,6\end{array}$ \\
\hline BMSG & $\begin{array}{l}\text { in Schilling } \\
\text { in \% }\end{array}$ & $\begin{array}{r}11148269 \\
100,0\end{array}$ & - & $\begin{array}{r}300000 \\
2,7\end{array}$ & - & - & - & - & $\begin{array}{r}1006585 \\
9,0\end{array}$ & $\begin{array}{r}6509498 \\
58,4\end{array}$ & $\begin{array}{r}959079 \\
8,6\end{array}$ & - & - & - & $\begin{array}{r}2373107 \\
21,3\end{array}$ \\
\hline BMAA & $\begin{array}{l}\text { in Schilling } \\
\text { in \% }\end{array}$ & & - & - & - & - & - & - & - & - & - & - & - & - & . \\
\hline BMJ & $\begin{array}{l}\text { in Schilling } \\
\text { in \% }\end{array}$ & $\begin{array}{r}850000 \\
100,0\end{array}$ & - & - & - & - & - & - & - & $\begin{array}{r}850000 \\
100,0\end{array}$ & - & - & - & - & . \\
\hline BML & $\begin{array}{l}\text { in Schilling } \\
\text { in \% }\end{array}$ & $\begin{array}{r}5929830 \\
100,0\end{array}$ & - & - & - & - & - & - & $\begin{array}{r}1243330 \\
21,0\end{array}$ & $\begin{array}{r}818000 \\
13,8\end{array}$ & $\begin{array}{r}192500 \\
3,2\end{array}$ & - & $\begin{array}{r}2548000 \\
43,0\end{array}$ & - & $\begin{array}{r}1128000 \\
19,0\end{array}$ \\
\hline BMF & $\begin{array}{l}\text { in Schilling } \\
\text { in } \%\end{array}$ & - & - & - & - & - & - & - & : & - & : & : & : & - & . \\
\hline BMLFUW & $\begin{array}{l}\text { in Schilling } \\
\text { in \% }\end{array}$ & $\begin{array}{r}58837324 \\
100,0\end{array}$ & $\begin{array}{r}4275533 \\
7,3\end{array}$ & $\begin{array}{r}19862142 \\
33,8\end{array}$ & $\begin{array}{r}4360290 \\
7,4\end{array}$ & $\begin{array}{r}1209000 \\
2,1\end{array}$ & - & - & $\begin{array}{r}2612003 \\
4,4\end{array}$ & $\begin{array}{r}3167705 \\
5,4\end{array}$ & $\begin{array}{r}14302618 \\
24,3\end{array}$ & $\begin{array}{r}437500 \\
0,7\end{array}$ & - & - & $\begin{array}{r}8610533 \\
14,6\end{array}$ \\
\hline BMWA & $\begin{array}{l}\text { in Schilling } \\
\text { in } \%\end{array}$ & $\begin{array}{r}11055630 \\
100,0\end{array}$ & $\begin{array}{r}2701000 \\
24,4\end{array}$ & $\begin{array}{r}367000 \\
3,3\end{array}$ & $\begin{array}{r}2917600 \\
26,4\end{array}$ & 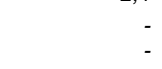 & $\begin{array}{r}57270 \\
0,5\end{array}$ & - & $\begin{array}{r}1120000 \\
10,1\end{array}$ & $\begin{array}{r}1145000 \\
10,4\end{array}$ & $\begin{array}{r}1500000 \\
13,6\end{array}$ & - & - & - & $\begin{array}{r}1247760 \\
11,3\end{array}$ \\
\hline INSGESAMT & $\begin{array}{l}\text { in Schilling } \\
\text { in } \%\end{array}$ & $\begin{array}{r}788066227 \\
100,0\end{array}$ & $\begin{array}{r}45476878 \\
5,8\end{array}$ & $\begin{array}{r}27700028 \\
3,5\end{array}$ & $\begin{array}{r}71979859 \\
9,1\end{array}$ & $\begin{array}{r}16894053 \\
2,1\end{array}$ & $\begin{array}{r}16721823 \\
2,1\end{array}$ & $\begin{array}{r}23176991 \\
2,9\end{array}$ & $\begin{array}{r}53454712 \\
6,8\end{array}$ & $\begin{array}{r}141092151 \\
18,0\end{array}$ & $\begin{array}{r}72074504 \\
9,1\end{array}$ & $\begin{array}{r}3839054 \\
0,5\end{array}$ & $\begin{array}{r}2548000 \\
0,3\end{array}$ & $\begin{array}{r}14987353 \\
1,9\end{array}$ & $\begin{array}{r}298120821 \\
37,9\end{array}$ \\
\hline
\end{tabular}


Auswertung der Faktendokumentation der Bundesdienststellen für 2000 (Stand: Juli 2001) einschließ lich "große" Globalförderungen ${ }^{1}$ )

\begin{tabular}{|c|c|c|c|c|c|c|c|}
\hline \multirow[b]{2}{*}{ RESSORTS } & \multirow[b]{2}{*}{$\begin{array}{l}\text { Teilbeträge } \\
2000\end{array}$} & \multicolumn{6}{|c|}{ davon für } \\
\hline & & $\begin{array}{c}1.0 \\
\text { Naturwissen- } \\
\text { schaften }\end{array}$ & $\begin{array}{c}2.0 \\
\text { Technische } \\
\text { Wissenschaften }\end{array}$ & $\begin{array}{c}3.0 \\
\text { Humanmedizin }\end{array}$ & $\begin{array}{l}4.0 \\
\text { Land- und } \\
\text { Forstwirtschaft, } \\
\text { Veterinärmedizin }\end{array}$ & $\begin{array}{c}5.0 \\
\text { Sozialwissen- } \\
\text { schaften }\end{array}$ & $\begin{array}{c}6.0 \\
\begin{array}{c}\text { Geisteswissen- } \\
\text { schaften }\end{array}\end{array}$ \\
\hline
\end{tabular}

\begin{tabular}{|c|c|}
\hline BKA & $\begin{array}{l}\text { in Schilling } \\
\text { in } \%\end{array}$ \\
\hline BMI & $\begin{array}{l}\text { in Schilling } \\
\text { in \% }\end{array}$ \\
\hline BMBWK & $\begin{array}{l}\text { in Schilling } \\
\text { in } \%\end{array}$ \\
\hline BMVIT & $\begin{array}{l}\text { in Schilling } \\
\text { in } \%\end{array}$ \\
\hline BMSG & $\begin{array}{l}\text { in Schilling } \\
\text { in } \%\end{array}$ \\
\hline BMAA & $\begin{array}{l}\text { in Schilling } \\
\text { in \% }\end{array}$ \\
\hline BMJ & $\begin{array}{l}\text { in Schilling } \\
\text { in } \%\end{array}$ \\
\hline BML & $\begin{array}{l}\text { in Schilling } \\
\text { in } \%\end{array}$ \\
\hline BMF & $\begin{array}{l}\text { in Schilling } \\
\text { in } \%\end{array}$ \\
\hline BMLFUW & $\begin{array}{l}\text { in Schilling } \\
\text { in } \%\end{array}$ \\
\hline BMWA & $\begin{array}{l}\text { in Schilling } \\
\text { in } \%\end{array}$ \\
\hline
\end{tabular}

\section{INSGESAMT in Schilling}

$$
\text { in \% }
$$

$$
\begin{array}{r}
391776 \\
100,0 \\
1197548208 \\
100,0 \\
2064405863 \\
100,0 \\
11148269 \\
100,0 \\
- \\
- \\
850000 \\
100,0 \\
5929830 \\
100,0 \\
- \\
- \\
58837324 \\
100,0 \\
11055630 \\
100,0
\end{array}
$$

3350166900

100,0

$$
\begin{array}{r}
496232590 \\
41,4 \\
547346361 \\
26,5 \\
2482324 \\
22,3 \\
- \\
- \\
- \\
- \\
1092500 \\
18,4 \\
- \\
- \\
21069277 \\
35,8 \\
5468200 \\
49,5
\end{array}
$$

1073691252
32,0
1225588221
36,6 $\begin{array}{r}100,0 \\ \hline\end{array}$

105116423

1108690912
53,7

53,7

$-$

$-$

2738000

46,2

5975680

10,2

2675430

24,2

138214769
11,5
164721150
8,0
1006585
9,0
-
-
-
-
743330
12,5
-
-
2612003
4,4
1020000
9,2

308317837

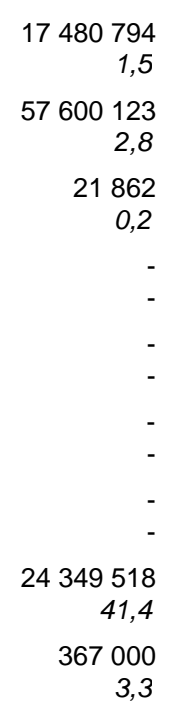

191289420

249214212

$$
105768224
$$

105768224

$$
7637498
$$$$
68,5
$$$$
850000
$$$$
100,0
$$$$
1356000
$$$$
22,9
$$

$$
\begin{array}{r}
3700541 \\
6,3 \\
1525000
\end{array}
$$$$
1525000
$$

80279093
3,9

1) d.h. einschließlich Globalförderungen für: Fonds zur Förderung der wissenschaftlichen Forschung, Forschungsförderungsfonds für die gewerbliche Wirtschaft, Ludwig Boltzmann-Gesellschaft, Österreichische Akademie der

Wissenschaften, Österreichisches Forschungszentrum Seibersdorf (insgesamt 2562100673 Schilling). Die in der Faktendokumentation 2000 nicht ausgewiesenen Förderungsmittel seitens des BMBWK an den FWF sowie seitens des BMVIT an den FFF wurden aus dem Bundesrechnungsabschluss 2000 ermittelt und entsprechend berücksichtigt. 
Auswertung der Faktendokumentation der Bundesdienststellen für 2000 (Stand: Juli 2001) ohne "große" Globalförderungen 1)

\begin{tabular}{|c|c|c|c|c|c|c|c|}
\hline \multirow[b]{2}{*}{ RESSORTS } & \multirow[b]{2}{*}{$\begin{array}{c}\text { Teilbeträge } \\
2000\end{array}$} & \multicolumn{6}{|c|}{ davon für } \\
\hline & & $\begin{array}{c}1.0 \\
\begin{array}{l}\text { Naturwissen- } \\
\text { schaften }\end{array}\end{array}$ & $\begin{array}{c}2.0 \\
\text { Technische } \\
\text { Wissenschaften }\end{array}$ & $\begin{array}{c}3.0 \\
\text { Humanmedizin }\end{array}$ & $\begin{array}{l}4.0 \\
\text { Land- und } \\
\text { Forstwirtschaft, } \\
\text { Veterinärmedizin }\end{array}$ & $\begin{array}{c}5.0 \\
\text { Sozialwissen- } \\
\text { schaften }\end{array}$ & $\begin{array}{c}6.0 \\
\text { Geisteswissen- } \\
\text { schaften }\end{array}$ \\
\hline
\end{tabular}

BKA

BMI

BMBWK

BMVIT

BMSG

BMAA

BMJ

BML

BMF

BMLFUW

BMWA in Schilling

in \%

in Schilling

$$
\text { in \% }
$$

in Schilling

$$
\text { in \% }
$$

in Schilling

$$
\text { in } \%
$$

in Schilling

$$
\text { in \% }
$$$$
\text { in Schilling }
$$$$
\text { in \% }
$$$$
\text { in Schilling }
$$$$
\text { in \% }
$$$$
\text { in Schilling }
$$

$$
\text { in \% }
$$$$
\text { in Schilling }
$$$$
\text { in \% }
$$$$
\text { in Schilling }
$$$$
\text { in } \%
$$$$
\text { in Schilling }
$$$$
\text { in } \%
$$

$$
\begin{array}{r}
391776 \\
100,0 \\
387860208 \\
100,0 \\
311993190 \\
100,0 \\
11148269 \\
100,0 \\
- \\
- \\
850000 \\
100,0 \\
5929830 \\
100,0 \\
- \\
- \\
58837324 \\
100,0 \\
11055630 \\
100,0
\end{array}
$$

788066227

100,0

Schilling

$$
\text { in } \%
$$

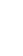

$\begin{array}{rr}- & 391776 \\ - & 100,0 \\ 82430504 & 29911613\end{array}$

28924608
7,5
8447550
2,7
1006585
9,0
-
-
-
-
743330
12,5
-
-
2612003
4,4
1020000
9,2

7596990
2,0
328936
0,1
21862
0,2
-
-
-
-
-
-
-
-
24349518
41,4
367000
3,3

129762789

109233704

57576847

2482324

167109860

53,5

22,3

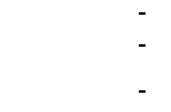

1092500

2738000

46,2
21069277
35,8
468200
49,5

5975680

10,2
675430

24,2

170119652
21,6

208802359

26,5

42754076

5,4

32664306

$\begin{array}{rr}4,1 & 216332232 \\ & 27,5\end{array}$

117393602

Quelle: Statistik Austria (Bundesanstalt Statistik Österreich)

1) d.h. ohne Globalförderungen für: Fonds zur Förderung der wissenschaftlichen Forschung, Forschungsförderungsfonds für die gewerbliche Wirtschaft, Ludwig Boltzmann-Gesellschaft, Österreichische Akademie der Wissenschaften, Österreichisches Forschungszentrum Seibersdorf (insgesamt 2562100673 Schilling). 
gegliedert nach Wissenschaftszweigen und Beschäftigtenkategorien

(VZÄ-Schätzung aufgrund des Stellenplanes 1. 1. 2002; 1 Planstelle = 1,0 VZÄ; auf der Basis der Koeffizienten aus der F\&E-Erhebung 1998)

Vollzeitäquivalente (VZ̈̈) für Lehre und Ausbildung, Forschung und experimentelle Entwicklung und Sonstige Tätigkeiten

\begin{tabular}{|c|c|c|c|c|c|c|c|c|c|c|c|c|c|c|c|c|c|c|c|c|c|c|c|c|}
\hline \multirow{3}{*}{ Wissenschaftszweige } & \multicolumn{4}{|c|}{ Professoren } & \multicolumn{4}{|c|}{$\begin{array}{c}\text { Assistenten, } \\
\text { Universitätsdozenten } \\
\end{array}$} & \multicolumn{4}{|c|}{$\begin{array}{c}\text { Sonstiges wissenschaftliches } \\
\text { Personal }\end{array}$} & \multicolumn{4}{|c|}{$\begin{array}{l}\text { Wissenschaftliches Personal } \\
\text { zusammen }\end{array}$} & \multicolumn{4}{|c|}{$\begin{array}{l}\text { Nichtwissenschaftliches } \\
\text { Personal } \\
\end{array}$} & \multicolumn{4}{|c|}{ INSGESAMT } \\
\hline & 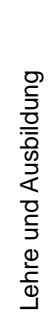 & 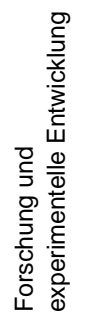 & 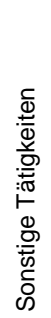 & 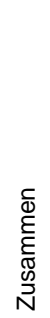 & 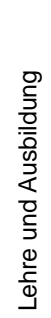 & 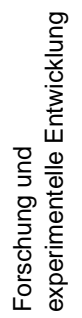 & 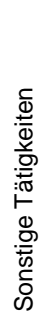 & 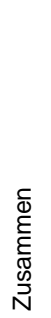 & 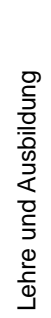 & 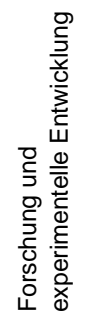 & 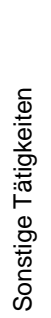 & 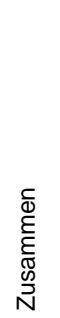 & 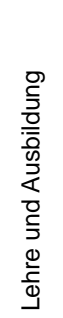 & 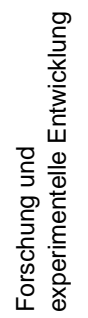 & 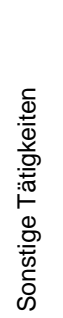 & 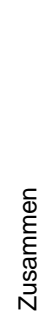 & 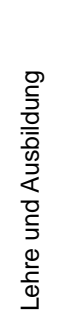 & 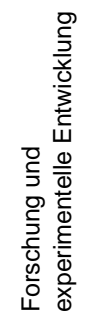 & 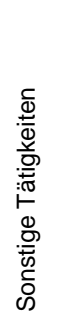 & 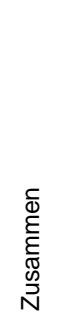 & 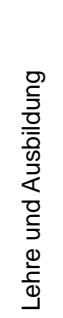 & 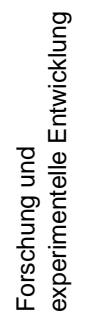 & 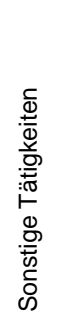 & 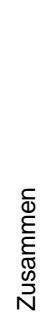 \\
\hline & & & & & & & & & & & & & $d \mathrm{i}$ & $n$ te & & & & & & & & & & \\
\hline
\end{tabular}

1.0 Naturwissenschaften

2.0 Technische Wissenschaften

3.0 Humanmedizin ohne Kliniken

Kliniken

einschließlich Kliniken

4.0 Land- und Forstwirtschaft,

Veterinärmedizin

1.0 bis $4.0 \quad$ Zwischensumme

ohne Kliniken einschließ lich Kliniken

5.0 Sozialwissenschaften

6.0 Geisteswissenschaften

5.0 und 6.0

Zwischensumme

$197,8 \quad 220,5$

$\begin{array}{llllll}43,7 & 462,0 & 611,3 & 778,9 & 109,8 & 1,500,0\end{array}$

$48,6 \quad 65,2$

$\begin{array}{ll}48,7 & 65,2 \\ 45,7\end{array}$

$\begin{array}{llllll}19,7 & 236,0 & 405,1 & -430,1 & 62,8 & 808,0\end{array}$

49,8

$\begin{array}{llllll}15,2 & 129,0 & 224,3 & 323,8 & 59,9 & 608,0\end{array}$

$\begin{array}{llllll}84,6 & 192,0 & 414,1 & 679,3 & 1.394,6 & 2.488,0\end{array}$

$\begin{array}{llllllll}94,3 & 126,9 & 99,8 & 321,0 & 638,4 & 1.003,1 & 1.454,5 & 3.096,0\end{array}$

$25,4 \quad 23,7$

8,9

$58,0 \quad 77,4$

$379,8 \quad 417,7$

$\begin{array}{rrrrrrr} & 87,5 & 885,0 & 1.318,1 & 1.632,4 & 278,5 & 3.229,0\end{array}$

$223,5 \quad 224,2$

$170,1 \quad 163,5$

$\begin{array}{llll}34,3 & 482,0 & 554,3 & 618,7\end{array}$

$97,0 \quad 1.270,0$

$27,9 \quad 361,5 \quad 309,1 \quad 329,7$

$63,3 \quad 702,1$

31,3

$7,4 \quad 20,4 \quad 7,8$

$13,3 \quad 36,1$

$5,4 \quad 11,9$

17,5

$\begin{array}{rrrrrrrrrrrrrr}7,8 & 35,6 & 280,3 & 409,4 & 82,9 & 772,6 & 189,8 & 433,8 & 136,3 & 759,9 & 470,1 & 843,2 & 219,2 & 1.532,5\end{array}$

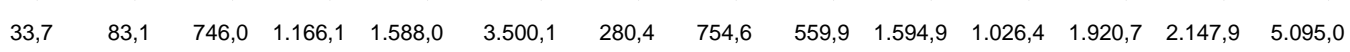

1.0 bis 6.0 INSGESAMT

ohne Kliniken einschließ lich Kliniken

$393,6 \quad 387,7$

$\begin{array}{llllll}3,4 & 20,7 & 108,2 & 135,2 & 58,3 & 301,7\end{array}$

$106,0 \quad 186,9$

$97,4 \quad 390,3 \quad 214,2 \quad 322,1 \quad 155,7 \quad 692,0$

$\begin{array}{llllllllll}773,4 & 805,4 & 149,7 & 1.728,5 & 2.181,5 & 2.580,8 & 438,8 & 5.201,1 & 375,7 & 290,3\end{array}$

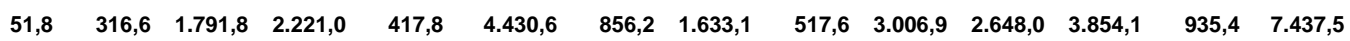

$\begin{array}{llllllllllllllll}99,8 & 186,6 & 77,7 & 364,1 & 2.257,5 & 2.977,7 & 1.922,9 & 7.158,1 & 946,8 & 1.953,9 & 941,2 & 3.841,9 & 3.204,3 & 4.931,6 & 2.864,1 & 11.000,0\end{array}$

$\begin{array}{llllllllllllllll}60,9 & 42,0 & 12,0 & 114,9 & 838,7 & 884,9 & 143,3 & 1.866,9 & 221,8 & 249,1 & 68,0 & 538,9 & 1.060,5 & 1.134,0 & 211,3 & 2.405,8\end{array}$

$32,7 \quad 433,9 \quad 1.538,8 \quad 1.455,5$

$255,2 \quad 3.249,5$

$165,2 \quad 168,9$

$\begin{array}{llllll}10,9 & 915,9 & 1.925,8 & 1.873,5 & 366,1 & 4.165,4\end{array}$

Stand: April 2002

uelle: Statistik Austria (Bundesanstalt Statistk

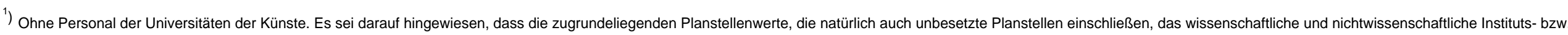

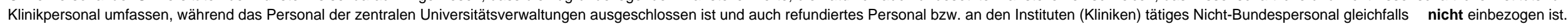


Tabelle 16: $\quad$ HOCHSCHULSEKTOR $^{1}$ )

AUSGABEN FÜR FORSCHUNG UND EXPERIMENTELLE ENTWICKLUNG IM J AHRE 1998 (in ATS)

(unter Einschluss der Gemeinkosten und Bauausgaben)

gegliedert nach Wissenschaftszweigen und Ausgabenarten

\begin{tabular}{|c|c|c|c|c|c|c|}
\hline \multirow[t]{2}{*}{ Wissenschaftszweige } & \multirow[t]{2}{*}{$\begin{array}{l}\text { F \& E be- } \\
\text { treibende } \\
\text { Erhe- } \\
\text { bungs- } \\
\text { einheiten }\end{array}$} & $\begin{array}{l}\text { Personal- } \\
\text { ausgaben }\end{array}$ & $\begin{array}{c}\text { Laufende } \\
\text { Sachausgaben }\end{array}$ & $\begin{array}{l}\text { Ausgaben für } \\
\text { Ausrüstungs- } \\
\text { investitionen }\end{array}$ & $\begin{array}{l}\text { Bauausgaben } \\
\text { und Ausgaben } \\
\text { für Liegen- } \\
\text { schaftsankäufe }\end{array}$ & Insgesamt \\
\hline & & \multicolumn{5}{|c|}{1000 ATS } \\
\hline
\end{tabular}

$\begin{array}{ll}1.0 & \text { Naturwissenschaften } \\ 2.0 & \text { Technische Wissenschaften } \\ 3.0 & \text { Humanmedizin } \\ 4.0 & \text { Land- und Forstwirtschaft, } \\ & \text { Veterinärmedizin }\end{array}$

1.0 bis $4.0 \quad$ Zwischensumme

$212 \quad 1661022$

$177 \quad 688520$

1925416

783525

353189

178178

1365130

1782910

172647

171752

4111379

152

229858

536362

51065

96784

1747007

47

3944530

5028213

755079

815378

87733

103327

191060

1490752

946139

6518965

5327538

Stand: April 2002

1015

621804

675374

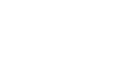

3913342

895178

10666906

\begin{tabular}{|c|c|c|c|}
\hline 5.0 & \multicolumn{2}{|c|}{ Sozialwissenschaften } & 198 \\
\hline 6.0 & \multicolumn{2}{|c|}{ Geisteswissenschaften } & 229 \\
\hline 5.0 & d 6.0 & Zwischensumme & 42 \\
\hline 1.0 & 6.0 & Insgesamt & 101 \\
\hline
\end{tabular}

1) Umfasst Universitäter

sitäten einschließlich Kliniken, Universitäten der Künste, Österreichisch

Quelle: STATISTIK AUSTRIA (Bundesanstalt Statistik Österreich)

1775966

1451187

3227153

13894059


Tabelle 17: $\quad$ HOCHSCHULSEKTOR ${ }^{1}$ )

FINANZIERUNG DER AUSGABEN FÜR FORSCHUNG UND EXPERIMENTELLE ENTWICKLUNG IM J AHRE 1998 (in ATS)

(unter Einschluss der Gemeinkosten und Bauausgaben)

gegliedert nach Wissenschaftszweigen und Finanzierungsbereichen

\begin{tabular}{|c|c|c|c|c|c|c|c|c|c|c|c|c|}
\hline & & & & & & & Finanzierur & sbereiche & & & & \\
\hline & & & & & & entlicher Sekt & & & & & & \\
\hline & Wissenschaftszweige & $\begin{array}{l}\text { treibende } \\
\text { Erhe- } \\
\text { bungs- } \\
\text { einheiten }\end{array}$ & $\begin{array}{l}\text { Unter- } \\
\text { nehmens- } \\
\text { sektor }\end{array}$ & Bund ${ }^{2}$ ) & Länder ${ }^{3}$ ) & $\begin{array}{c}\text { Gemein- } \\
\text { den }^{3} \text { ) }\end{array}$ & Sonstige $^{2}$ ) & $\begin{array}{c}\text { Zu- } \\
\text { sammen }\end{array}$ & $\begin{array}{l}\text { grivater } \\
\text { nütziger } \\
\text { Sektor }\end{array}$ & $\begin{array}{c}\text { interna- } \\
\text { tionaler } \\
\text { Organi- } \\
\text { sationen } \\
\text { (ohne EU) }\end{array}$ & EU & $\begin{array}{c}\text { Ins- } \\
\text { gesamt }\end{array}$ \\
\hline & & & & & & & 100 & & & & & \\
\hline 1.0 & Naturwissenschaften & 212 & 50538 & 3328388 & 53927 & - & 515498 & 3897813 & 8682 & 25933 & 128413 & 4111379 \\
\hline 2.0 & Technische Wissenschaften & 177 & 92494 & 1363941 & 28279 & - & 145059 & 1537279 & 11061 & 51028 & 55145 & 1747007 \\
\hline 3.0 & Humanmedizin & 152 & 66428 & 3429863 & 100609 & - & 236728 & 3767200 & 16389 & 38795 & 24530 & 3913342 \\
\hline 4.0 & Land- und Forstwirtschaft, & & & & & & & & & & & \\
\hline & Veterinärmedizin & 47 & 17604 & 716791 & 18948 & - & 99360 & 835099 & 15649 & 9159 & 17667 & 895178 \\
\hline $1.0 \mathrm{bis}$ & Zwischensumme & 588 & 227064 & 8838983 & 201763 & - & 996645 & 10037391 & 51781 & 124915 & 225755 & 10666906 \\
\hline 5.0 & Sozialwissenschaften & 198 & 11313 & 1693970 & 17610 & - & 37047 & 1748627 & 4749 & 3252 & 8025 & 1775966 \\
\hline 6.0 & Geisteswissenschaften & 229 & 4397 & 1319330 & 14789 & 335 & 99286 & 1433740 & 4808 & 3235 & 5007 & 1451187 \\
\hline 5.0 un & Zwischensumme & 427 & 15710 & 3013300 & 32399 & 335 & 136333 & 3182367 & 9557 & 6487 & 13032 & 3227153 \\
\hline $1.0 \mathrm{bis}$ & Insgesamt & 1015 & 242774 & 11852283 & 234162 & 335 & 1132978 & 13219758 & 61338 & 131402 & 238787 & 13894059 \\
\hline
\end{tabular}

Stand: April 2002

Quelle: STATISTIK AUSTRIA(Bundesanstalt Statistik Österreich)

1) Umfasst Universitäten einschließlich Kliniken, Universitäten der Künste, Österreichische Akademie der Wissenschaften und Versuchsanstalten an den Höheren Technischen Bundeslehranstalten.

${ }^{2}$ ) Die Mittel der Forschungsförderungsfonds und des ITF sind unter „Sonstige“ enthalten.

${ }^{3}$ ) Länder einschließlich Wien. Gemeinden ohne Wien. 
Tabelle 18: $\quad$ SEKTOR STAAT ${ }^{1}$ )

AUSGABEN FÜR FORSCHUNG UND EXPERIMENTELLE ENTWICKLUNG IM J AHRE 1998 (in ATS)

gegliedert nach Wissenschaftszweigen und Ausgabenarten

\begin{tabular}{|c|c|c|c|c|c|c|}
\hline \multirow[t]{2}{*}{ Wissenschaftszweige } & \multirow[t]{2}{*}{$\begin{array}{c}\text { F \& E be- } \\
\text { treibende } \\
\text { Erhe- } \\
\text { bungs- } \\
\text { einheiten }\end{array}$} & $\begin{array}{l}\text { Personal- } \\
\text { ausgaben }\end{array}$ & $\begin{array}{c}\text { Laufende } \\
\text { Sachausgaben }\end{array}$ & $\begin{array}{l}\text { Ausgaben für } \\
\text { Ausrüstungs- } \\
\text { investitionen }\end{array}$ & $\begin{array}{l}\text { Bauausgaben } \\
\text { und Ausgaben } \\
\text { für Liegen- } \\
\text { schaftsankäufe }\end{array}$ & Insgesamt \\
\hline & & \multicolumn{5}{|c|}{1000 ATS } \\
\hline
\end{tabular}

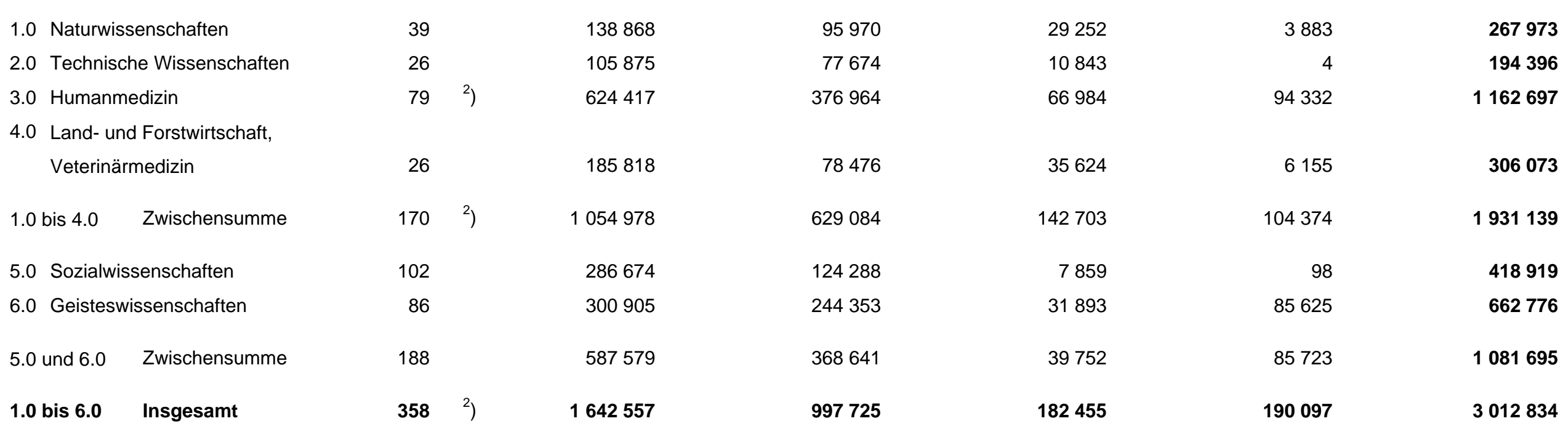

Stand: April 2002

Quelle: STATISTIK AUSTRIA (Bundesanstalt Statistik Österreich)

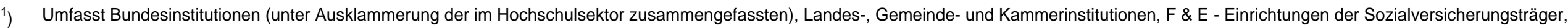

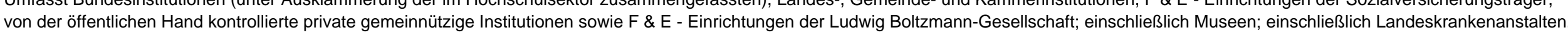

2) Anzahl der Erhebungseinheiten ohne Landeskrankenanstalten. 
Tabelle 19: $\quad$ SEKTOR STAAT ${ }^{1}$ )

FINANZIERUNG DER AUSGABEN FÜR FORSCHUNG UND EXPERIMENTELLE ENTWICKLUNG IM J AHRE 1998 (in ATS)

gegliedert nach Wissenschaftszweigen und Finanzierungsbereichen

\begin{tabular}{|c|c|c|c|c|c|c|c|c|c|c|c|}
\hline \multirow{4}{*}{ Wissenschaftszweige } & \multirow{4}{*}{$\begin{array}{c}\text { F \& E be- } \\
\text { treibende } \\
\text { Erhe- } \\
\text { bungs- } \\
\text { einheiten }\end{array}$} & \multicolumn{10}{|c|}{ Finanzierungsbereiche } \\
\hline & & \multirow[b]{2}{*}{$\begin{array}{l}\text { Unter- } \\
\text { nehmens- } \\
\text { sektor }\end{array}$} & \multicolumn{5}{|c|}{ Öffentlicher Sektor } & \multirow[b]{2}{*}{$\begin{array}{l}\text { Privater } \\
\text { gemein- } \\
\text { nütziger } \\
\text { Sektor }\end{array}$} & \multirow{2}{*}{$\begin{array}{c}\text { Ausland } \\
\text { einschl. } \\
\text { interna- } \\
\text { tionaler } \\
\text { Organi- } \\
\text { sationen } \\
\text { (ohne EU) }\end{array}$} & \multirow[b]{2}{*}{$\mathrm{EU}$} & \multirow[b]{2}{*}{$\begin{array}{c}\text { Ins- } \\
\text { gesamt }\end{array}$} \\
\hline & & & Bund $^{2}$ ) & Länder ${ }^{3}$ ) & $\begin{array}{c}\text { Gemein- } \\
\operatorname{den}^{3} \text { ) }\end{array}$ & Sonstige $^{2}$ ) & $\begin{array}{c}\text { Zu- } \\
\text { sammen }\end{array}$ & & & & \\
\hline & & \multicolumn{10}{|c|}{1000 ATS } \\
\hline
\end{tabular}

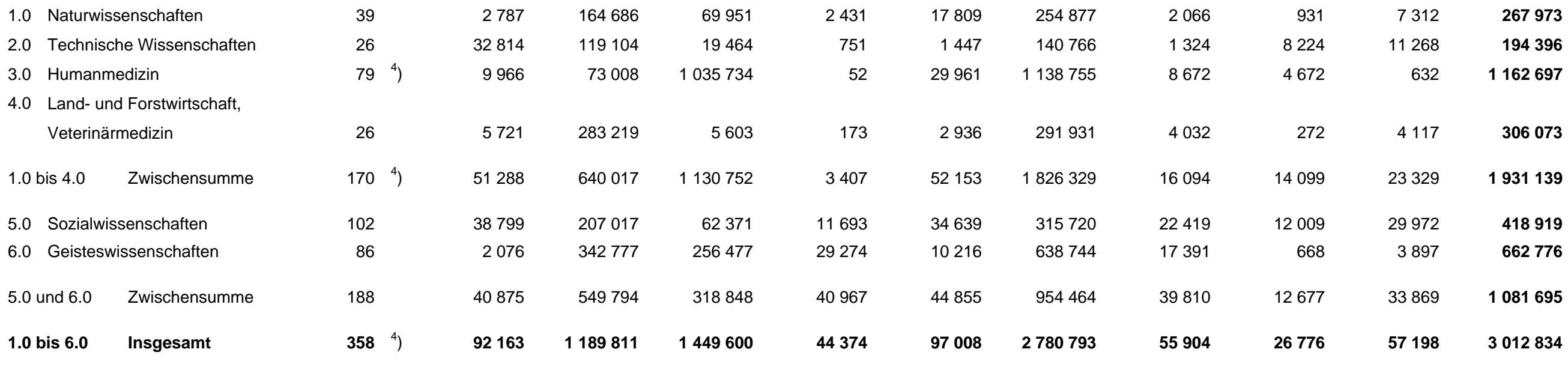

Stand: April 2002

Quelle: STATISTIK AUSTRIA (Bundesanstalt Statistik Österreich)

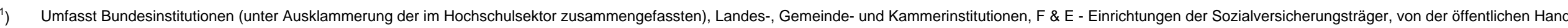
kontrollierte private gemeinnützige Institutionen sowie F \& E - Einrichtungen der Ludwig Boltzmann-Gesellschaft; einschließlich Museen; einschließlich Landeskrankenanstalten.

2) Die Mittel der Forschungsförderungsfonds und des ITF sind unter "Sonstige“ enthalten.

3) Länder einschließlich Wien. Gemeinden ohne Wien.

${ }^{4}$ ) Anzahl der Erhebungseinheiten ohne Landeskrankenanstalten. 
Tabelle 20: $\quad$ SEKTOR STAAT ${ }^{1}$ )

AUSGABEN FÜR FORSCHUNG UND EXPERIMENTELLE ENTWICKLUNG IM J AHRE 1998 (in ATS)

gegliedert nach Rechtsträgern und Ausgabenarten

\begin{tabular}{|c|c|c|c|c|c|c|}
\hline \multirow[t]{2}{*}{ Rechtsträger } & \multirow[t]{2}{*}{$\begin{array}{l}\text { F \& E be- } \\
\text { treibende } \\
\text { Erhe- } \\
\text { bungs- } \\
\text { einheiten }\end{array}$} & $\begin{array}{l}\text { Personal- } \\
\text { ausgaben }\end{array}$ & $\begin{array}{c}\text { Laufende } \\
\text { Sachausgaben }\end{array}$ & $\begin{array}{l}\text { Ausgaben für } \\
\text { Ausrüstungs- } \\
\text { investitionen }\end{array}$ & $\begin{array}{l}\text { Bauausgaben } \\
\text { und Ausgaben } \\
\text { für Liegen- } \\
\text { schaftsankäufe }\end{array}$ & Insgesamt \\
\hline & & \multicolumn{5}{|c|}{1000 ATS } \\
\hline
\end{tabular}

Bund

Länder (einschließlich Wien)

Gemeinden (ohne Wien)

Kammern

Sozialversicherungsträger

Ploe öffentlich ${ }^{4}$ )

Institute u. Forschungsstellen der LBG

Insgesamt
71

$42^{2}$ )

7

$6^{3}$ )

$\left.1^{3}\right)$

123

108

$\left.358^{2}\right)$
467175

708477

24795

11475

355332

75303

1642557

Stand: April 2002
253478

467675

15598

1401

230227

29346

997725
84394

64028

436

240

16483

16874

182455
31424

836471

139773

1379953

40829

13116

602157

140308

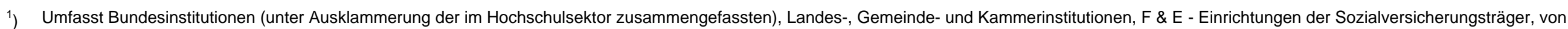
der öffentlichen Hand kontrollierte private gemeinnützige Institutionen sowie F \& E - Einrichtungen der Ludwig Boltzmann-Gesellschaft; einschließlich Museen; einschließlich Landeskrankenanstalten.

$\left.{ }^{2}\right)$ Anzahl der Erhebungseinheiten ohne Landeskrankenanstalten.

${ }^{3}$ ) Aus Geheimhaltungsgründen können die Daten nur gemeinsam ausgewiesen werden.

$\left.{ }^{4}\right)$ Private Institutionen ohne Erwerbscharakter, die hauptsächlich vom öffentlichen Sektor finanziert und/oder kontrolliert werden. 
Tabelle 21: SEKTOR STAAT $^{1}$ )

FINANZIERUNG DER AUSGABEN FÜR FORSCHUNG UND EXPERIMENTELLE ENTWICKLUNG IM J AHRE 1998 (in ATS)

(unter Einschluss der Gemeinkosten und Bauausgaben im Hochschulsektor)

gegliedert nach Rechtsträgern und Finanzierungsbereichen

\begin{tabular}{|c|c|c|c|c|c|c|c|c|c|c|c|}
\hline \multirow{4}{*}{ Rechtsträger } & \multirow{4}{*}{$\begin{array}{c}\text { F \& E be- } \\
\text { treibende } \\
\text { Erhe- } \\
\text { bungs- } \\
\text { einheiten }\end{array}$} & \multicolumn{10}{|c|}{ Finanzierungsbereiche } \\
\hline & & \multirow[b]{2}{*}{$\begin{array}{l}\text { Unter- } \\
\text { nehmens- } \\
\text { sektor }\end{array}$} & \multicolumn{5}{|c|}{ Öffentlicher Sektor } & \multirow[b]{2}{*}{$\begin{array}{l}\text { Privater } \\
\text { gemein- } \\
\text { nütziger } \\
\text { Sektor }\end{array}$} & \multirow{2}{*}{$\begin{array}{c}\text { Ausland } \\
\text { einschl. } \\
\text { interna- } \\
\text { tionaler } \\
\text { Organi- } \\
\text { sationen } \\
\text { (ohne EU) }\end{array}$} & \multirow[b]{2}{*}{ EU } & \multirow[b]{2}{*}{$\begin{array}{c}\text { Ins- } \\
\text { gesam }\end{array}$} \\
\hline & & & Bund $^{2}$ ) & Länder ${ }^{3}$ ) & $\begin{array}{c}\text { Gemein- } \\
\left.\operatorname{den}^{3}\right)\end{array}$ & Sonstige ${ }^{2}$ ) & $\begin{array}{c}\text { Zu- } \\
\text { sammen }\end{array}$ & & & & \\
\hline & & \multicolumn{10}{|c|}{1000 ATS } \\
\hline
\end{tabular}

Bund

71

5368

799856

234

238

818401

7839

627

4236

836471

Länder (einschließlich Wien)

$\left.42^{4}\right)$

94

1368097

131

253

1370400

4718

4141

600

1379953

Gemeinden (ohne Wien)

7

12

28810

40829

$-$

40829

Kammern

Sozialversicherungsträger

$6^{5}$ )

$1^{5}$ )

Ploe öffentlich ${ }^{6}$ )

Institute $u$. Forschungsstellen der LBG

123

73647

302130

61768

14812

13116

$-$

13116

108

92163

1189811

1449600

44374

38637

417347

43097

250

21163

46903

602157

$383 \quad 26929 \quad 120700$

$97008 \quad 2780793$

55904

26776

57198

Stand: April 2002

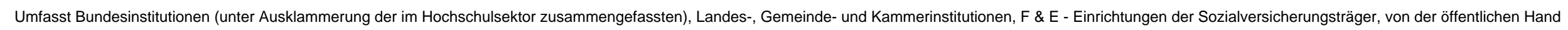
kontrollierte private gemeinnützige Institutionen sowie F \& E - Einrichtungen der Ludwig Boltzmann-Gesellschaft; einschließlich Museen; einschließlich Landeskrankenanstalten.

) Die Mittel der Forschungsförderungsfonds und des ITF sind unter "Sonstige" enthalten.

$\left.{ }^{3}\right)$ Länder einschließlich Wien. Gemeinden ohne Wien.

$\left.{ }^{4}\right)$ Anzahl der Erhebungseinheiten ohne Landeskrankenanstalten.

5) Aus Geheimhaltungsgründen können die Daten nur gemeinsam ausgewiesen werden.

${ }^{6}$ ) Private Institutionen ohne Erwerbscharakter, die hauptsächlich vom öffentlichen Sektor finanziert und/oder kontrolliert werden. 
Tabelle 22: PRIVATER GEMEINNÜTZIGER SEKTOR ${ }^{1}$ )

AUSGABEN FÜR FORSCHUNG UND EXPERIMENTELLE ENTWICKLUNG IM J AHRE 1998 (in ATS)

gegliedert nach Wissenschaftszweigen und Ausgabenarten

\begin{tabular}{|c|c|c|c|c|c|c|}
\hline \multirow[t]{2}{*}{ Wissenschaftszweige } & \multirow[t]{2}{*}{$\begin{array}{l}\text { F \& E be- } \\
\text { treibende } \\
\text { Erhe- } \\
\text { bungs- } \\
\text { einheiten }\end{array}$} & $\begin{array}{l}\text { Personal- } \\
\text { ausgaben }\end{array}$ & $\begin{array}{c}\text { Laufende } \\
\text { Sachausgaben }\end{array}$ & $\begin{array}{l}\text { Ausgaben für } \\
\text { Ausrüstungs- } \\
\text { investitionen }\end{array}$ & $\begin{array}{l}\text { Bauausgaben } \\
\text { und Ausgaben } \\
\text { für Liegen- } \\
\text { schaftsankäufe }\end{array}$ & Insgesamt \\
\hline & & \multicolumn{5}{|c|}{1000 ATS } \\
\hline
\end{tabular}
1.0 Naturwissenschaften
2.0 Technische Wissenschaften
3.0 Humanmedizin
4.0 Land- und Forstwirtschaft, Veterinärmedizin

\section{0 bis $4.0 \quad$ Zwischensumme}

5.0 Sozialwissenschaften

6.0 Geisteswissenschaften

5.0 und $6.0 \quad$ Zwischensumme

1.0 bis $6.0 \quad$ Insgesamt

Stand: April 2002

$\begin{array}{rrr}9490 & 3184 & 720 \\ 6586 & 4197 & 238 \\ 20875 & 17496 & 2625\end{array}$

36951

38084

706

38790

75741
37785

664

38449

63326
720

2625

3583

1564

22

1586

5169
13394

11021

40996

$\left.{ }^{1}\right)$ Private Institutionen ohne Erwerbscharakter, deren Status ein vorwiegend privater oder privatrechtlicher, konfessioneller oder sonstiger nicht öffentlicher ist.

$\left.{ }^{2}\right)$ Aus Geheimhaltungsgründen können die Daten nur gemeinsam ausgewiesen werden. 
Tabelle 23: PRIVATER GEMEINNÜTZIGER SEKTOR ${ }^{1}$ )

FINANZIERUNG DER AUSGABEN FÜR FORSCHUNG UND EXPERIMENTELLE ENTWICKLUNG IM J AHRE 1998 (in ATS)

gegliedert nach Wissenschaftszweigen und Finanzierungsbereichen

\begin{tabular}{|c|c|c|c|c|c|c|c|c|c|c|c|}
\hline \multirow{4}{*}{ Wissenschaftszweige } & \multirow{4}{*}{$\begin{array}{c}\text { F \& E be- } \\
\text { treibende } \\
\text { Erhe- } \\
\text { bungs- } \\
\text { einheiten }\end{array}$} & \multicolumn{10}{|c|}{ Finanzierungsbereiche } \\
\hline & & \multirow[b]{2}{*}{$\begin{array}{l}\text { Unter- } \\
\text { nehmens- } \\
\text { sektor }\end{array}$} & \multicolumn{5}{|c|}{ Öffentlicher Sektor } & \multirow[b]{2}{*}{$\begin{array}{c}\text { Privater } \\
\text { gemein- } \\
\text { nütziger } \\
\text { Sektor }\end{array}$} & \multirow{2}{*}{$\begin{array}{c}\text { Ausland } \\
\text { einschl. } \\
\text { interna- } \\
\text { tionaler } \\
\text { Organi- } \\
\text { sationen } \\
\text { (ohne EU) }\end{array}$} & \multirow[b]{2}{*}{$\mathrm{EU}$} & \multirow[b]{2}{*}{$\begin{array}{c}\text { Ins- } \\
\text { gesamt }\end{array}$} \\
\hline & & & Bund $^{2}$ ) & Länder ${ }^{3}$ ) & $\begin{array}{c}\text { Gemein- } \\
\operatorname{den}^{3} \text { ) }\end{array}$ & Sonstige $^{2}$ ) & $\begin{array}{c}\text { Zu- } \\
\text { sammen }\end{array}$ & & & & \\
\hline & & \multicolumn{10}{|c|}{1000 ATS } \\
\hline
\end{tabular}
1.0 Naturwissenschaften
2.0 Technische Wissenschaften
3.0 Humanmedizin
4.0 Land- und Forstwirtschaft, Veterinärmedizin

$11-7004$

$10 \quad 6372$

1911

893

$\left.3^{4}\right) \quad 2070$

668

339

64

- $\quad 2868$

$$
\left.1^{4}\right)
$$

1.0 bis $4.0 \quad$ Zwischensumme

15446

$2579 \quad 1232$

32900

17471

2832

5

6.0 Geisteswissenschaften

5.0 und $6.0 \quad$ Zwischensumme

28

45

$507 \quad 141$

32945

17978

2973

20557

4205

48391

-
421
356

2868

1503
2099
26303

1815

204

13394

1.0 bis $6.0 \quad$ Insgesamt

53

Stand: April 2002

Quelle: STATISTIK AUSTRIA (Bundesanstalt Statistik Österreich)

1) Private Institutionen ohne Erwerbscharakter, deren Status ein vorwiegend privater oder privatrechtlicher, konfessioneller oder sonstiger nicht öffentlicher ist.

2) Die Mittel der Forschungsförderungsfonds und des ITF sind unter "Sonstige" enthalten.

3) Länder einschließlich Wien. Gemeinden ohne Wien.

${ }^{4}$ ) Aus Geheimhaltungsgründen können die Daten nur gemeinsam ausgewiesen werden. 
Tabelle 24: UNTERNEHMENSSEKTOR OHNE FIRMENEIGENER BEREICH (KOOPERATIVER BEREICH ${ }^{1}$ ), BEREICH DER ZIVILTECHNIKER)

AUSGABEN FÜR FORSCHUNG UND EXPERIMENTELLE ENTWICKLUNG IM J AHRE 1998 (in ATS)

gegliedert nach Wissenschaftszweigen und Ausgabenarten

\begin{tabular}{|c|c|c|c|c|c|c|}
\hline \multirow[t]{2}{*}{ Wissenschaftszweige } & \multirow[t]{2}{*}{$\begin{array}{c}\text { F \& E be- } \\
\text { treibende } \\
\text { Erhe- } \\
\text { bungs- } \\
\text { einheiten }\end{array}$} & $\begin{array}{l}\text { Personal- } \\
\text { ausgaben }\end{array}$ & $\begin{array}{c}\text { Laufende } \\
\text { Sachausgaben }\end{array}$ & $\begin{array}{l}\text { Ausgaben für } \\
\text { Ausrüstungs- } \\
\text { investitionen }\end{array}$ & $\begin{array}{l}\text { Bauausgaben } \\
\text { und Ausgaben } \\
\text { für Liegen- } \\
\text { schaftsankäufe }\end{array}$ & Insgesamt \\
\hline & & \multicolumn{5}{|c|}{1000 ATS } \\
\hline
\end{tabular}

1.0 Naturwissenschaften

2.0 Technische Wissenschaften

3.0 Humanmedizin

4.0 Land- und Forstwirtschaft,

Veterinärmedizin

1.0 bis $4.0 \quad$ Zwischensumme

5.0 Sozialwissenschaften

6.0 Geisteswissenschaften

5.0 und 6.0 Zwischensumme

1.0 bis $6.0 \quad$ Insgesamt

7

$29^{2}$ )

$1^{2}$ )

4

41

279826

1119402

12119

1411347

10072

10072

45

1421419

Stand: April 2002

1) Einschließlich Österreichisches Forschungszentrum Seibersdorf.

${ }^{2}$ ) Aus Geheimhaltungsgründen können die Daten nur gemeinsam ausgewiesen werden.

\section{5}

811876

3868

981229

5260

5260

986489
27344

110267

1409

139020

568

568

139588
5019

22617

506

28142

-

$-$

$-$

28142

Quelle: STATISTIK AUSTRIA(Bundesanstalt Statistik Österreich)
477674

2064162

17902

2559738

15900

15900

2575638 
Tabelle 25: UNTERNEHMENSSEKTOR OHNE FIRMENEIGENER BEREICH

(KOOPERATIVER BEREICH ${ }^{1}$ ), BEREICH DER ZIVILTECHNIKER)

FINANZIERUNG DER AUSGABEN FÜR FORSCHUNG UND EXPERIMENTELLE ENTWICKLUNG IM J AHRE 1998 (in ATS)

gegliedert nach Wissenschaftszweigen und Finanzierungsbereichen

\begin{tabular}{|c|c|c|c|c|c|c|c|c|c|c|c|}
\hline \multirow[b]{3}{*}{ Wissenschaftszweige } & \multirow[b]{3}{*}{$\begin{array}{l}\mathrm{F} \& \mathrm{E} \text { be- } \\
\text { treibende } \\
\text { Erhe- } \\
\text { bungs- } \\
\text { einheiten }\end{array}$} & \multicolumn{10}{|c|}{ Finanzierungsbereiche } \\
\hline & & \multirow[b]{2}{*}{$\begin{array}{l}\text { Unter- } \\
\text { nehmens- } \\
\text { sektor }\end{array}$} & \multicolumn{5}{|c|}{ Öffentlicher Sektor } & \multirow[b]{2}{*}{$\begin{array}{l}\text { Privater } \\
\text { gemein- } \\
\text { nütziger } \\
\text { Sektor }\end{array}$} & \multirow{2}{*}{$\begin{array}{l}\text { Ausland } \\
\text { einschl. } \\
\text { interna- } \\
\text { tionaler } \\
\text { Organi- } \\
\text { sationen } \\
\text { (ohne EU) }\end{array}$} & \multirow[b]{2}{*}{ EU } & \multirow[b]{2}{*}{$\begin{array}{c}\text { Ins- } \\
\text { gesamt }\end{array}$} \\
\hline & & & Bund $^{2}$ ) & Länder ${ }^{3}$ ) & $\begin{array}{l}\text { Gemein- } \\
\operatorname{den}^{3} \text { ) }\end{array}$ & Sonstige $^{2}$ ) & $\begin{array}{c}\text { Zu- } \\
\text { sammen }\end{array}$ & & & & \\
\hline
\end{tabular}

1.0 Naturwissenschaften

2.0 Technische Wissenschaften

3.0 Humanmedizin

4.0 Land- und Forstwirtschaft, Veterinärmedizin

1.0 bis $4.0 \quad$ Zwischensumme

5.0 Sozialwissenschaften

6.0 Geisteswissenschaften

5.0 und $6.0 \quad$ Zwischensumme

1.0 bis $6.0 \quad$ Insgesamt

7

$29^{4}$ )

19368

323204

$1^{4}$ )

357428

65922

\section{1}

150069

4

41

\section{4}

$-$

4

45

391020

393784
33491

379681

$\begin{array}{rll}115 & 33491 & 379681 \\ 12437 & 75291 & 303719\end{array}$

- $\quad 62736$

$258 \quad 1350205$

15889

52552

477674

\section{Stand: April 2002}

1) Einschließlich Österreichisches Forschungszentrum Seibersdorf.

2) Die Mittel der Forschungsförderungsfonds und des ITF sind unter "Sonstige" enthalten.

$\left.{ }^{3}\right)$ Länder einschließlich Wien. Gemeinden ohne Wien.

${ }^{4}$ ) Aus Geheimhaltungsgründen können die Daten nur gemeinsam ausgewiesen werden.
172976

12555

116373

595

172946

12555

6466

6944

690344

1232

115248

1125

5344

5344

695688

Quelle: STATISTIK AUSTRIA (Bundesanstalt Statistik Österreich)
559738

15900

15900

575638 
Tabelle 26: FORSCHUNG UND EXPERIMENTELLE ENTWICKLUNG IN SÄMTLICHEN DURCHFÜHRUNGSSEKTOREN (OHNE FIRMENEIGENER BEREICH) $\left(\right.$ HOCHSCHULSEKTOR $^{1}$ ), SEKTOR STAAT ${ }^{2}$ ), PRIVATER GEMEINNÜTZIGER SEKTOR ${ }^{3}$ ), UNTERNEHMENSSEKTOR $\left.{ }^{4}\right)$ )

AUSGABEN FÜR FORSCHUNG UND EXPERIMENTELLE ENTWICKLUNG IM J AHRE 1998 (in ATS)

gegliedert nach Wissenschaftszweigen und Ausgabenarten

\begin{tabular}{|c|c|c|c|c|c|c|}
\hline \multirow[t]{2}{*}{ Wissenschaftszweige } & \multirow[t]{2}{*}{$\begin{array}{c}\text { F \& E be- } \\
\text { treibende } \\
\text { Erhe- } \\
\text { bungs- } \\
\text { einheiten }\end{array}$} & $\begin{array}{l}\text { Personal- } \\
\text { ausgaben }\end{array}$ & $\begin{array}{l}\text { Laufende } \\
\text { Sachausgaben }\end{array}$ & $\begin{array}{l}\text { Ausgaben für } \\
\text { Ausrüstungs- } \\
\text { investitionen }\end{array}$ & $\begin{array}{l}\text { Bauausgaben } \\
\text { und Ausgaben } \\
\text { für Liegen- } \\
\text { schaftsankäufe }\end{array}$ & Insgesamt \\
\hline & & \multicolumn{5}{|c|}{1000 ATS } \\
\hline
\end{tabular}

1.0 Naturwissenschaften

$\begin{array}{llll}269 & & 2089206 & 2190055 \\ 242 & & 1850932 & 1616623 \\ \left.2355^{5}\right) & 2077495 & 2229880\end{array}$

410505
277046
264478

\section{4 \\ 115317}

4870420

2.0 Technische Wissenschaften

3.0 Humanmedizin

78

430173

626845

88356

3859918

4.0 Land- und Forstwirtschaft,

Veterinärmedizin

$824^{5}$ )

6447806

6663403

1040385

84554

5262928

1.0 bis $4.0 \quad$ Zwischensumme

327

5.0 Sozialwissenschaften

320

1096034

982711

97724

135242

232966

1903102

2019449

8566505

8467255

1273351

$1471^{5}$ )

8467255

Stand: April 2002

1) Umfasst Universitäten einschließlich Kliniken, Universitäten

Gemeinkosten und Bauausgaben sind eingeschlossen.

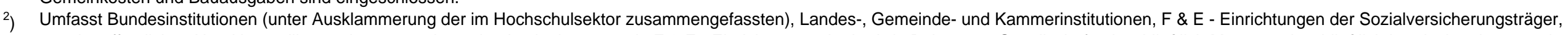

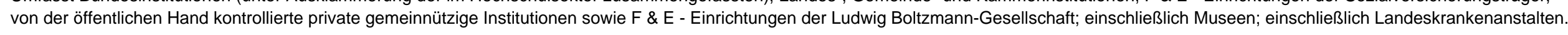

$\left.{ }^{3}\right)$ Private Institutionen ohne Erwerbscharakter, deren Status ein vorwiegend privater oder privatrechtlicher, konfessioneller oder sonstiger nicht öffentlicher ist.

${ }^{4}$ ) Umfasst den Kooperativen Bereich einschließlich Österreichisches Forschungszentrum Seibersdorf sowie den Bereich der Ziviltechniker. Ohne Firmeneigener Bereich.

5) Anzahl der Erhebungseinheiten ohne Landeskrankenanstalten. 
FORSCHUNG UND EXPERIMENTELLE ENTWICKLUNG IN SÄMTLICHEN DURCHFÜHRUNGSSEKTOREN (OHNE FIRMENEIGENER BEREICH)

(HOCHSCHULSEKTOR ${ }^{1}$ ), SEKTOR STAAT ${ }^{2}$ ), PRIVATER GEMEINNÜTZIGER SEKTOR ${ }^{3}$ ), UNTERNEHMENSSEKTOR $\left.{ }^{4}\right)$ )

FINANZIERUNG DER AUSGABEN FÜR FORSCHUNG UND EXPERIMENTELLE ENTWICKLUNG IM J AHRE 1998 (in ATS)

gegliedert nach Wissenschaftszweigen und Finanzierungsbereichen

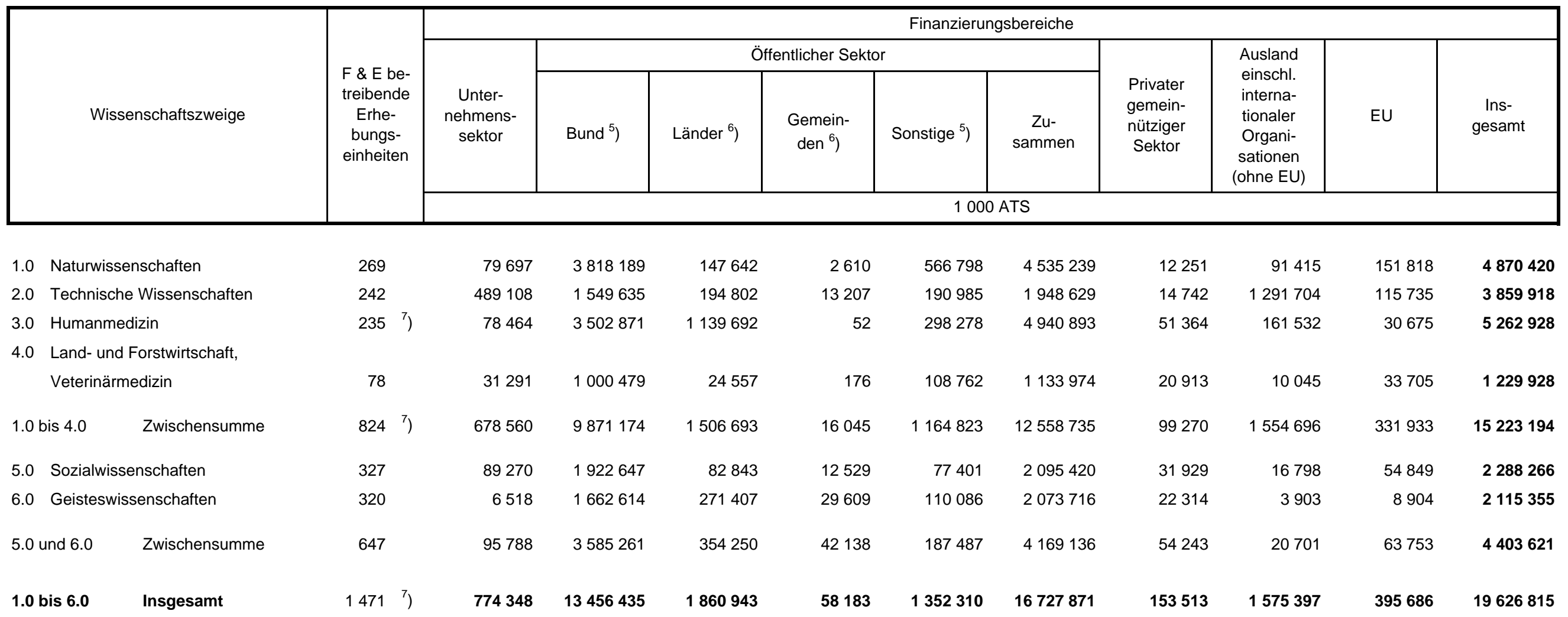

Stand: April 2002

Quelle: STATISTIK AUSTRIA(Bundesanstalt Statistik Österreich)

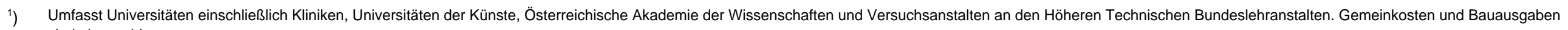
sind eingeschlossen.

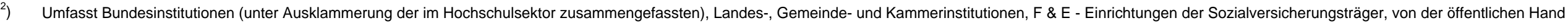
kontrollierte private gemeinnützige Institutionen sowie F \& E - Einrichtungen der Ludwig Boltzmann-Gesellschaft; einschließlich Museen; einschließlich Landeskrankenanstalten

${ }^{3}$ ) Private Institutionen ohne Erwerbscharakter, deren Status ein vorwiegend privater oder privatrechtlicher, konfessioneller oder sonstiger nicht öffentlicher ist.

$\left.{ }^{4}\right)$ Umfasst den Kooperativen Bereich einschließlich Österreichisches Forschungszentrum Seibersdorf sowie den Bereich der Ziviltechniker. Ohne Firmeneigener Bereich.

Die Mittel der Forschungsförderungsfonds und des ITF sind unter „Sonstige“ enthalten.

Länder einschließlich Wien. Gemeinden ohne Wien.

${ }^{7}$ ) Anzahl der Erhebungseinheiten ohne Landeskrankenanstalten. 
Tabelle 28: AUSGABEN FÜR FORSCHUNG UND EXPERIMENTELLE ENTWICKLUNG IM J AHRE 1998 (in ATS)

(unter Einschluss der Gemeinkosten und Bauausgaben im Hochschulsektor)

gegliedert nach Durchführungssektoren/ Erhebungsbereichen und Ausgabenarten

\begin{tabular}{|c|c|c|c|c|c|c|}
\hline \multirow[t]{2}{*}{ Sektoren / Bereiche } & \multirow[t]{2}{*}{$\begin{array}{l}\text { F \& E be- } \\
\text { treibende } \\
\text { Erhe- } \\
\text { bungs- } \\
\text { einheiten }\end{array}$} & $\begin{array}{l}\text { Personal- } \\
\text { ausgaben }\end{array}$ & $\begin{array}{l}\text { Laufende } \\
\text { Sachausgaben }\end{array}$ & $\begin{array}{l}\text { Ausgaben für } \\
\text { Ausrüstungs- } \\
\text { investitionen }\end{array}$ & $\begin{array}{l}\text { Bauausgaben } \\
\text { und Ausgaben } \\
\text { für Liegen- } \\
\text { schaftsankäufe }\end{array}$ & Insgesamt \\
\hline & & \multicolumn{5}{|c|}{1000 ATS } \\
\hline \multicolumn{6}{|l|}{ davon: } & 13894059 \\
\hline Universitäten (ohne Kliniken) & 822 & 4285864 & 5072846 & 865268 & 558750 & 10782728 \\
\hline Universitätskliniken & 77 & 797696 & 1132332 & 41788 & 536217 & 2508033 \\
\hline Universitäten der Künste & 56 & 51114 & 64379 & 8110 & 5905 & 129508 \\
\hline Akademie der Wissenschaften & 56 & 190960 & 249276 & 30973 & 545 & 471754 \\
\hline Versuchsanstalten an HTL's & 4 & 1904 & 132 & - & - & 2036 \\
\hline $\begin{array}{l}\text { Sektor Staat }{ }^{1} \text { ) } \\
\text { davon: }\end{array}$ & $\left.358^{2}\right)$ & 1642557 & 997725 & 182455 & 190097 & 3012834 \\
\hline Ohne Landeskrankenanstalten & 358 & 1085105 & 654735 & 132949 & 114641 & 1987430 \\
\hline Landeskrankenanstalten ${ }^{3}$ ) & . & 557452 & 342990 & 49506 & 75456 & 1025404 \\
\hline Privater gemeinnütziger Sektor ${ }^{4}$ ) & 53 & 75741 & 63326 & 5169 & 48 & 144284 \\
\hline $\begin{array}{l}\text { Unternehmenssektor } \\
\text { davon: }\end{array}$ & 1317 & 16540469 & 10517899 & 2379121 & 294088 & 29731577 \\
\hline Kooperativer Bereich ${ }^{5}$ ) & 25 & 1406847 & 976288 & 137162 & 28082 & 2548379 \\
\hline Ziviltechniker & 20 & 14572 & 10201 & 2426 & 60 & 27259 \\
\hline Kraftwerksgesellschaften ${ }^{6}$ ) & & & . & . & . & \\
\hline Firmeneigener Bereich & 1272 & 15119050 & 9531410 & 2239533 & 265946 & 27155939 \\
\hline Insgesamt & $2743^{2}$ ) & 23586305 & 18097915 & 3512884 & 1585650 & 46782754 \\
\hline
\end{tabular}

Stand: April 2002

Quelle: STATISTIK AUSTRIA (Bundesanstalt Statistik Österreich)

$\left.{ }^{1}\right)$ Umfasst Bundesinstitutionen (unter Ausklammerung der im Hochschulsektor zusammengefassten), Landes-, Gemeinde- und Kammerinstitutionen, $\mathrm{F}$ \& $\mathrm{E}$ - Einrichtungen der

Sozialversicherungsträger, von der öffentlichen Hand kontrollierte private gemeinnützige Institutionen sowie F \& E - Einrichtungen der Ludwig Boltzmann-Gesellschaft; einschließlich Museen einschließlich Landeskrankenanstalten.

$\left.{ }^{2}\right)$ Anzahl der Erhebungseinheiten ohne Krankenanstalten.

$\left.{ }^{3}\right)$ Schätzung von STATISTIK AUSTRIA unter Heranziehung der Meldungen der Ämter der Landesregierungen.

$\left.{ }^{4}\right)$ Private Institutionen ohne Erwerbscharakter, deren Status ein vorwiegend privater oder privatrechtlicher, konfessioneller oder sonstiger nicht öffentlicher ist.

$\left.{ }^{5}\right)$ Einschließlich Österreichisches Forschungszentrum Seibersdorf.

${ }^{6}$ ) Seit der F \& E-Erhebung 1998 sind die F \& E - Einrichtungen dieses Subsektors im Subsektor „Firmeneigener Bereich“ enthalten. Aus Gründen der Vergleichbarkeit des Unternehmenssektors mit den Ergebnissen aus früheren F \& E - Erhebungen wurde der Subsektor „Kraftwerksgesellschaften“ in der Vorspalte weiter angeführt. 
FINANZIERUNG DER AUSGABEN FÜR FORSCHUNG UND EXPERIMENTELLE ENTWICKLUNG IM J AHRE 1998 (in ATS)

(unter Einschluss der Gemeinkosten und Bauausgaben im Hochschulsektor)

gegliedert nach Durchführungssektoren/ Erhebungsbereichen und Finanzierungsbereichen

\begin{tabular}{|c|c|c|c|c|c|c|c|c|c|c|c|}
\hline \multirow{4}{*}{$\begin{array}{l}\text { F \& E durchgeführt in den } \\
\text { Sektoren / Bereichen }\end{array}$} & \multirow{4}{*}{$\begin{array}{c}\mathrm{F} \& \mathrm{E} \text { be- } \\
\text { treibende } \\
\text { Erhe- } \\
\text { bungs- } \\
\text { einheiten }\end{array}$} & \multicolumn{10}{|c|}{ Finanzierungsbereiche } \\
\hline & & \multirow[b]{2}{*}{$\begin{array}{l}\text { Unter- } \\
\text { nehmens- } \\
\text { sektor }\end{array}$} & \multicolumn{5}{|c|}{ Öffentlicher Sektor } & \multirow[b]{2}{*}{$\begin{array}{l}\text { Privater } \\
\text { gemein- } \\
\text { nütziger } \\
\text { Sektor }\end{array}$} & \multirow{2}{*}{$\begin{array}{l}\text { Ausland } \\
\text { einschl. } \\
\text { interna- } \\
\text { tionaler } \\
\text { Organi- } \\
\text { sationen } \\
\text { (ohne EU) }\end{array}$} & \multirow[b]{2}{*}{ EU } & \multirow[b]{2}{*}{$\begin{array}{c}\text { Ins- } \\
\text { gesamt }\end{array}$} \\
\hline & & & Bund $^{1}$ ) & Länder ${ }^{2}$ ) & $\begin{array}{l}\text { Gemein- } \\
\operatorname{den}^{2} \text { ) }\end{array}$ & Sonstige ${ }^{1}$ ) & $\begin{array}{c}\text { Zu- } \\
\text { sammen }\end{array}$ & & & & \\
\hline & & \multicolumn{10}{|c|}{1000 ATS } \\
\hline $\begin{array}{l}\text { Hochschulsektor } \\
\text { davon: }\end{array}$ & 1015 & 242774 & 11852283 & 234162 & 335 & 1132978 & 13219758 & 61338 & 131402 & 238787 & 13894059 \\
\hline Universitäten (ohne Kliniken) & 822 & 180935 & 9092958 & 139574 & - & 1002037 & 10234569 & 54662 & 92273 & 220289 & 10782728 \\
\hline Universitätskliniken & 77 & 51903 & 2234500 & 86346 & - & 92866 & 2413712 & 3454 & 28866 & 10098 & 2508033 \\
\hline Universitäten der Künste & 56 & 4012 & 114404 & 1841 & - & 6908 & 123153 & 2029 & 44 & 270 & 129508 \\
\hline Akademie der Wissenschaften & 56 & 3888 & 410421 & 6401 & 335 & 31167 & 448324 & 1193 & 10219 & 8130 & 471754 \\
\hline Versuchsanstalten an HTL's & 4 & 2036 & - & - & - & - & - & - & - & - & 2036 \\
\hline $\begin{array}{l}\text { Sektor Staat }{ }^{3} \text { ) } \\
\text { davon: }\end{array}$ & $\left.358^{4}\right)$ & 92163 & 1189811 & 1449600 & 44374 & 97008 & 2780793 & 55904 & 26776 & 57198 & 3012834 \\
\hline $\begin{array}{l}\text { Ohne Landeskrankenanstalten } \\
\text { Landeskrankenanstalten }\end{array}$ & 358 & 92163 & 1189811 & $\begin{array}{r}424196 \\
1025404\end{array}$ & 44374 & 97008 & 1755389 & 55904 & 26776 & 57198 & $\begin{array}{l}1987430 \\
1025404\end{array}$ \\
\hline $\begin{array}{l}\text { Landeskrankenanstalten } \\
\text { Privater gemeinnütziger Sektor }{ }^{5} \text { ) }\end{array}$ & 53 & 48391 & 20557 & 4205 & 919 & 5951 & 31632 & 34781 & 3655 & 25825 & $\begin{array}{r}1025404 \\
144284\end{array}$ \\
\hline $\begin{array}{l}\text { Unternehmenssektor } \\
\text { davon: }\end{array}$ & 1317 & 19134719 & 489920 & 271576 & 24178 & 858291 & 1643965 & 15853 & 8649160 & 287880 & 29731577 \\
\hline Kooperativer Bereich ${ }^{6}$ ) & 25 & 380853 & 386891 & 170625 & 11671 & 115395 & 684582 & 1234 & 1412795 & 68915 & 2548379 \\
\hline $\begin{array}{l}\text { Ziviltechniker } \\
\text { Kraftwerksgesellschaften }{ }^{7} \text { ) }\end{array}$ & 20 & 10167 & 6893 & 2351 & 884 & 978 & 11106 & 256 & 769 & 4961 & 27259 \\
\hline Firmeneigener Bereich & 1272 & 18743699 & 96136 & 98600 & 11623 & 741918 & 948277 & 14363 & 7235596 & 214004 & 27155939 \\
\hline Insgesamt & $\left.27433^{4}\right)$ & 19518047 & 13552571 & 1959543 & 69806 & 2094228 & 17676148 & 167876 & 8810993 & 609690 & 46782754 \\
\hline
\end{tabular}

Stand: April 2002

Quelle: STATISTIK AUSTRIA (Bundesanstalt Statistik Österreich)

1) Die Mittel der Forschungsförderungsfonds und des ITF sind unter "Sonstige“ enthalten.

${ }^{2}$ ) Länder einschließlich Wien. Gemeinden ohne Wien.

$\left.{ }^{3}\right)$ Umfasst Bundesinstitutionen (unter Ausklammerung der im Hochschulsektor zusammengefassten), Landes-, Gemeinde- und Kammerinstitutionen, F \& E - Einrichtungen der Sozialversicherungsträger, von der öffentlichen Hand

kontrollierte private gemeinnützige Institutionen sowie F \& E - Einrichtungen der Ludwig Boltzmann-Gesellschaft; einschließlich Museen; einschließlich Landeskrankenanstalten

$\left.{ }^{4}\right)$ Anzahl der Erhebungseinheiten ohne Landeskrankenanstalten

$\left.{ }^{5}\right)$ Private Institutionen ohne Erwerbscharakter, deren Status ein vorwiegend privater oder privatrechtlicher, konfessioneller oder sonstiger nicht öffentlicher ist.

$\left.{ }^{6}\right)$ Einschließlich Österreichisches Forschungszentrum Seibersdorf.

7) Seit der F \& E - Erhebung 1998 sind die F \& E - Einrichtungen dieses Subsektors im Subsektor „Firmeneigener Bereich“ enthalten. Aus Gründen der Vergleichbarkeit des Unternehmenssektors mit den Ergebnissen aus früheren F \& E - Erhebungen wurde der Subsektor „Kraftwerksgesellschaften“ in der Vorspalte weiter angeführt. 


\begin{tabular}{|c|c|c|c|c|c|}
\hline \multirow{2}{*}{ Bundesländer } & $\begin{array}{c}\text { Personal- } \\
\text { ausgaben }\end{array}$ & $\begin{array}{c}\text { Laufende } \\
\text { Sachausgaben }\end{array}$ & $\begin{array}{c}\text { Ausgaben für } \\
\text { Ausrüstungs- } \\
\text { investitionen }\end{array}$ & $\begin{array}{c}\text { Bauausgaben } \\
\text { und Ausgaben } \\
\text { für Liegen- } \\
\text { schaftsankäufe }\end{array}$ & Insgesamt \\
\cline { 2 - 6 } & \multicolumn{3}{|c|}{1000 ATS } \\
\hline
\end{tabular}

\begin{tabular}{|c|c|c|c|c|c|}
\hline Burgenland & 65395 & 31266 & 10516 & 1490 & 108667 \\
\hline Kärnten & 700259 & 747022 & 200791 & 34522 & 1682594 \\
\hline Niederösterreich & 1476205 & 878276 & 342851 & 18431 & 2715763 \\
\hline Oberösterreich & 2828126 & 2121770 & 419622 & 63934 & 5433452 \\
\hline Salzburg & 669772 & 477978 & 94839 & 33602 & 1276191 \\
\hline Steiermark & 4038992 & 3212066 & 640098 & 359895 & 8251051 \\
\hline Tirol & 1630578 & 1322163 & 360230 & 255502 & 3568473 \\
\hline Vorarlberg & 582985 & 280630 & 157373 & 20141 & 1041129 \\
\hline Wien & 11593993 & 9026744 & 1286564 & 798133 & 22705434 \\
\hline INSGESAMT & 23586305 & 18097915 & 3512884 & 1585650 & 46782754 \\
\hline
\end{tabular}


Tabelle 31: SOZIO-ÖKONOMISCHE ZIELSETZUNGEN

BESCHÄFTIGTE IN FORSCHUNG UND EXPERIMENTELLE ENTWICKLUNG (in Kopfzahlen) IM J AHRE 1998

gegliedert nach Durchführungssektoren/Erhebungsbereichen und sozio-ökonomischen Zielsetzungen (ÖSTAT-Klassifikation)

\begin{tabular}{|c|c|c|c|c|c|c|c|c|c|c|c|c|c|c|}
\hline \multirow[b]{2}{*}{ Sektoren / Bereiche } & \multirow[b]{2}{*}{ Kopfzahlen } & \multicolumn{13}{|c|}{ davon für } \\
\hline & & $\begin{array}{c}\text { Förderung } \\
\text { der Erfor- } \\
\text { schung der } \\
\text { Erde, der } \\
\text { Meere, der } \\
\text { Atmos- } \\
\text { phäre und } \\
\text { des Welt- } \\
\text { raumes }\end{array}$ & $\begin{array}{l}\text { Förderung } \\
\text { der Land- } \\
\text { und Forst- } \\
\text { wirtschaft }\end{array}$ & $\begin{array}{c}\text { Förderung } \\
\text { von Handel, } \\
\text { Gewerbe } \\
\text { und } \\
\text { Industrie }\end{array}$ & \begin{tabular}{|c|} 
Förderung \\
der \\
Erzeugung, \\
Speiche- \\
rung und \\
Verteilung \\
von Energie
\end{tabular} & $\begin{array}{l}\text { Förderung } \\
\text { des } \\
\text { Transport-, } \\
\text { Verkehrs- } \\
\text { und Nach- } \\
\text { richten- } \\
\text { wesens }\end{array}$ & $\begin{array}{l}\text { Förderung } \\
\text { des } \\
\text { Unterrichts- } \\
\text { und } \\
\text { Bildungs- } \\
\text { wesens }\end{array}$ & $\begin{array}{l}\text { Förderung } \\
\text { des } \\
\text { Gesund- } \\
\text { heits- } \\
\text { wesens }\end{array}$ & $\begin{array}{l}\text { Förderung } \\
\text { der } \\
\text { sozialen } \\
\text { und sozio- } \\
\text { ökonomi- } \\
\text { schen Ent- } \\
\text { wicklung }\end{array}$ & $\begin{array}{c}\text { Förderung } \\
\text { des } \\
\text { Umwelt- } \\
\text { schutzes }\end{array}$ & $\begin{array}{l}\text { Förderung } \\
\text { der Stadt- } \\
\text { und } \\
\text { Raum- } \\
\text { planung }\end{array}$ & $\begin{array}{l}\text { Förderung } \\
\text { der } \\
\text { Landes- } \\
\text { vertei- } \\
\text { digung }\end{array}$ & $\begin{array}{c}\text { Förderung } \\
\text { anderer } \\
\text { Zielset- } \\
\text { zungen }\end{array}$ & $\begin{array}{c}\text { Förderung } \\
\text { der all- } \\
\text { gemeinen } \\
\text { Erwei- } \\
\text { terung des } \\
\text { Wissens }\end{array}$ \\
\hline
\end{tabular}

\begin{tabular}{|c|c|c|c|c|c|c|c|c|c|c|c|c|c|c|}
\hline $\begin{array}{l}\text { Hochschulsektor } \\
\text { davon: }\end{array}$ & 21933 & 1192 & 654 & 2503 & 377 & 304 & 369 & 7132 & 1772 & 502 & 265 & - & 256 & 6607 \\
\hline Universitäten (ohne Kliniken) & 16291 & 1126 & 654 & 2441 & 377 & 304 & 339 & 2266 & 1663 & 501 & 249 & - & 256 & 6115 \\
\hline Universitätskliniken & 4810 & - & - & - & - & - & - & 4810 & - & - & - & - & - & - \\
\hline Kunsthochschulen & 239 & - & - & 19 & - & - & 30 & - & 59 & - & 3 & - & - & 128 \\
\hline Akademie der Wissenschaften & 582 & 66 & - & 32 & - & - & & 56 & 50 & 1 & 13 & - & - & 364 \\
\hline Versuchsanstalten an HTL's & 11 & - & - & 11 & - & - & - & - & - & - & - & - & - & - \\
\hline Sektor Staat ${ }^{1}$ ) & 5734 & 396 & 1393 & 412 & 59 & 42 & 69 & 515 & 848 & 115 & 71 & 44 & 24 & 1746 \\
\hline Privater gemeinnütziger Sektor ${ }^{2}$ ) & 349 & 12 & 9 & 20 & 9 & 29 & 21 & 74 & 84 & 42 & - & - & - & 49 \\
\hline $\begin{array}{l}\text { Unternehmenssektor } \\
\text { davon: }\end{array}$ & 3045 & - & - & 2761 & 1 & 12 & - & 196 & 10 & 33 & 31 & - & - & 1 \\
\hline Kooperativer Bereich ${ }^{3}$ ) & 2915 & - & - & 2697 & - & - & - & 196 & 9 & 12 & - & - & - & 1 \\
\hline Ziviltechniker & 130 & - & - & 64 & 1 & 12 & - & - & 1 & 21 & 31 & - & - & - \\
\hline Kraftwerksgesellschaften ${ }^{4}$ ) & & $\cdot$ & - & . & . & ${ }^{\circ}$ & . & . & . & . & . & . & . & . \\
\hline Firmeneigener Bereich ${ }^{5}$ ) & · & . & . & $\cdot$ & . & . & - & · & $\cdot$ & · & . & . & . & . \\
\hline Insgesamt ${ }^{6}$ ) & 31061 & 1600 & 2056 & 5696 & 446 & 387 & 459 & 7917 & 2714 & 692 & 367 & 44 & 280 & 8403 \\
\hline
\end{tabular}

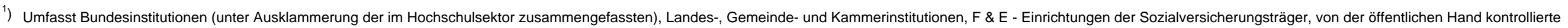
private gemeinnützige Institutionen sowie F \& E - Einrichtungen der Ludwig Boltzmann-Gesellschaft; einschließlich Museen; ohne Landeskrankenanstalten .

$\left.{ }^{2}\right)$ Private Institutionen ohne Erwerbscharakter, deren Status ein vorwiegend privater oder privatrechtlicher, konfessioneller oder sonstiger nicht öffentlicher ist.

$\left.{ }^{3}\right)$ Einschließlich Österreichisches Forschungszentrum Seibersdorf.

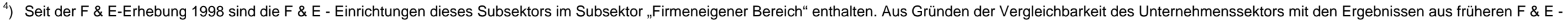
Erhebungen wurde der Subsektor "Kraftwerksgesellschaften“ in der Vorspalte weiter angeführt.

5) Auf die F\&E-Einrichtungen des firmeneigenen Bereichs ist eine Zuordnung nach der Systematik der sozio-ökonomischen Zielsetzungen aus sachlichen und definitorischen Gründen (Frascati-Handbuch) nicht anwendbar.

$\left.{ }^{6}\right)$ Ohne Firmeneigener Bereich. 
BESCHÄFTIGTE IN FORSCHUNG UND EXPERIMENTELLE ENTWICKLUNG (in Vollzeitäquivalenten) IM J AHRE 1998 gegliedert nach Durchführungssektoren/Erhebungsbereichen und sozio-ökonomischen Zielsetzungen (ÖSTAT-Klassifikation)

\begin{tabular}{|c|c|c|c|c|c|c|c|c|c|c|c|c|c|c|}
\hline \multirow[b]{2}{*}{ Sektoren / Bereiche } & \multirow[b]{2}{*}{$\begin{array}{l}\text { Vollzeitäqivalente } \\
\text { für } F \& E\end{array}$} & \multicolumn{13}{|c|}{ davon für } \\
\hline & & 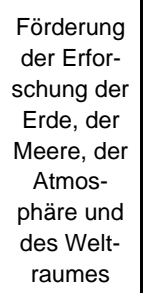 & $\begin{array}{c}\text { Förderung } \\
\text { der Land- } \\
\text { und Forst- } \\
\text { wirtschaft }\end{array}$ & \begin{tabular}{|c|} 
Förderung \\
von Handel, \\
Gewerbe \\
und \\
Industrie
\end{tabular} & $\begin{array}{c}\text { Förderung } \\
\text { der } \\
\text { Erzeugung, } \\
\text { Speiche- } \\
\text { rung und } \\
\text { Verteilung } \\
\text { von Energie }\end{array}$ & $\begin{array}{c}\text { Förderung } \\
\text { des } \\
\text { Transport-, } \\
\text { Verkehrs- } \\
\text { und Nach- } \\
\text { richten- } \\
\text { wesens }\end{array}$ & $\begin{array}{c}\text { Förderung } \\
\text { des } \\
\text { Unterrichts- } \\
\text { und } \\
\text { Bildungs- } \\
\text { wesens }\end{array}$ & $\begin{array}{c}\text { Förderung } \\
\text { des } \\
\text { Gesund- } \\
\text { heits- } \\
\text { wesens }\end{array}$ & $\begin{array}{c}\text { Förderung } \\
\text { der } \\
\text { sozialen } \\
\text { und sozio- } \\
\text { ökonomi- } \\
\text { schen Ent- } \\
\text { wicklung }\end{array}$ & $\begin{array}{c}\text { Förderung } \\
\text { des } \\
\text { Umwelt- } \\
\text { schutzes }\end{array}$ & $\begin{array}{c}\text { Förderung } \\
\text { der Stadt- } \\
\text { und } \\
\text { Raum- } \\
\text { planung }\end{array}$ & $\begin{array}{c}\text { Förderung } \\
\text { der } \\
\text { Landes- } \\
\text { vertei- } \\
\text { digung }\end{array}$ & $\begin{array}{c}\text { Förderung } \\
\text { anderer } \\
\text { Zielset- } \\
\text { zungen }\end{array}$ & $\begin{array}{l}\text { Förderung } \\
\text { der all- } \\
\text { gemeinen } \\
\text { Erwei- } \\
\text { terung des } \\
\text { Wissens }\end{array}$ \\
\hline $\begin{array}{l}\text { Hochschulsektor } \\
\text { davon: }\end{array}$ & 8670,1 & 535,5 & 310,6 & 1071,9 & 188,1 & 146,8 & 116,1 & 2362,9 & 654,7 & 239,7 & 87,2 & - & 105,8 & 2851,0 \\
\hline Universitäten (ohne Kliniken) & 6963,3 & 494,2 & 310,6 & 1045,5 & 188,1 & 146,8 & 105,3 & 1050,2 & 611,4 & 239,7 & 78,8 & - & 105,8 & 2586,9 \\
\hline Universitätskliniken & 1277,7 & - & - & - & - & - & - & 1277,7 & - & - & - & - & - & \\
\hline Kunsthochschulen & 74,0 & - & - & 3,2 & - & - & 10,8 & - & 16,3 & - & 0,7 & - & - & 43,0 \\
\hline Akademie der Wissenschaften & 351,6 & 41,3 & - & 19,6 & - & - & - & 35,0 & 27,0 & - & 7,6 & - & - & 221,1 \\
\hline Versuchsanstalten an HTL's & 3,5 & - & - & 3,5 & - & - & - & - & - & - & - & - & - & \\
\hline Sektor Staat ${ }^{1}$ ) & 2104,4 & 128,0 & 396,1 & 153,8 & 23,4 & 15,4 & 30,9 & 169,3 & 403,9 & 42,5 & 41,6 & 25,1 & 12,6 & 661,9 \\
\hline Privater gemeinnütziger Sektor ${ }^{2}$ ) & 148,4 & - & 5,1 & 7,5 & 0,8 & 14,1 & 10,4 & 39,7 & 36,0 & 18,2 & - & - & - & 16,6 \\
\hline $\begin{array}{l}\text { Unternehmenssektor } \\
\text { davon: }\end{array}$ & 1857,6 & - & - & 1733,0 & 0,4 & 2,8 & - & 101,7 & 5,6 & 4,1 & 10,0 & - & - & 0,1 \\
\hline Kooperativer Bereich ${ }^{3}$ ) & 1834,4 & - & - & 1726,3 & - & - & - & 101,7 & 5,3 & 1,2 & - & - & - & 0,1 \\
\hline Ziviltechniker & 23,2 & - & - & 6,8 & 0,4 & 2,8 & - & - & 0,3 & 2,9 & 10,0 & - & - & \\
\hline Kraftwerksgesellschaften ${ }^{4}$ ) & . & . & . & . & . & . & . & . & . & . & . & . & . & \\
\hline Firmeneigener Bereich ${ }^{5}$ ) & . & - & . & . & . & . & . & . & . & . & . & . & . & 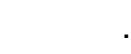 \\
\hline Insgesamt ${ }^{6}$ ) & 12780,6 & 663,5 & 711,9 & 2966,2 & 212,7 & 179,0 & 157,3 & 2673,6 & 1100,2 & 304,6 & 138,7 & 25,1 & 118,3 & 3529,6 \\
\hline
\end{tabular}

Stand: April 2002

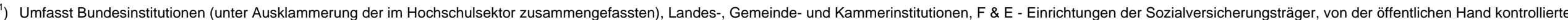
private gemeinnützige Institutionen sowie $\mathrm{F} \& \mathrm{E}$ - Einrichtungen der Ludwig Boltzmann-Gesellschaft; einschließlich Museen; ohne Landeskrankenanstalten.

$\left.{ }^{2}\right)$ Private Institutionen ohne Erwerbscharakter, deren Status ein vorwiegend privater oder privatrechtlicher, konfessioneller oder sonstiger nicht offentlicher ist.

) Einschließlich Österreichisches Forschungszentrum Seibersdorf.

$\left.{ }^{4}\right)$ Seit der F \& E-Erhebung 1998 sind die F \& E - Einrichtungen dieses Subsektors im Subsektor „Firmeneigener Bereich“ enthalten. Aus Gründen der Vergleichbarkeit des Unternehmenssektors mit den Ergebnissen aus früheren F \& E Erhebungen wurde der Subsektor Kraftwerksgesellschaften“ in der Vorspalte weiter angeführt.

$\left.{ }^{5}\right)$ Auf die F\&E-Einrichtungen des firmeneigenen Bereichs ist eine Zuordnung nach der Systematik der sozio-ökonomischen Zielsetzungen aus sachlichen und definitorischen Gründen (Frascati-Handbuch) nicht anwendbar.

$\left.{ }^{6}\right)$ Ohne Firmeneigener Bereich.

Rundungsdifferenzen. 
Tabelle 33: SOZIO-ÖKONOMISCHE ZIELSETZUNGEN

AUSGABEN FÜR FORSCHUNG UND EXPERIMENTELLE ENTWICKLUNG IM J AHRE 1998 (in ATS)

gegliedert nach Durchführungssektoren/Erhebungsbereichen und sozio-ökonomischen Zielsetzungen (ÖSTAT-Klassifikation)

\begin{tabular}{|c|c|c|c|c|c|c|c|c|c|c|c|c|c|c|}
\hline \multirow{3}{*}{ Sektoren / Bereiche } & \multirow[b]{2}{*}{$\begin{array}{l}\text { Ausgaben } \\
\text { für F\&E } \\
\text { insgesamt }\end{array}$} & \multicolumn{13}{|c|}{ davon für } \\
\hline & & $\begin{array}{c}\text { Förderung } \\
\text { der Erfor- } \\
\text { schung der } \\
\text { Erde, der } \\
\text { Meere, der } \\
\text { Atmos- } \\
\text { phäre und } \\
\text { des Welt- } \\
\text { raumes }\end{array}$ & $\begin{array}{l}\text { Förderung } \\
\text { der Land- } \\
\text { und Forst- } \\
\text { wirtschaft }\end{array}$ & \begin{tabular}{|c|} 
Förderung \\
von Handel, \\
Gewerbe \\
und \\
Industrie
\end{tabular} & 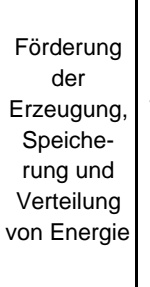 & $\begin{array}{c}\begin{array}{c}\text { Förderung } \\
\text { des }\end{array} \\
\text { Transport-, } \\
\text { Verkehrs- } \\
\text { und Nach- } \\
\text { richten- } \\
\text { wesens }\end{array}$ & \begin{tabular}{|c|} 
Förderung \\
des \\
Unterrichts- \\
und \\
Bildungs- \\
wesens
\end{tabular} & $\begin{array}{c}\text { Förderung } \\
\text { des } \\
\text { Gesund- } \\
\text { heits- } \\
\text { wesens }\end{array}$ & $\begin{array}{c}\text { Förderung } \\
\text { der } \\
\text { sozialen } \\
\text { und sozio- } \\
\text { ökonomi- } \\
\text { schen Ent- } \\
\text { wicklung }\end{array}$ & $\begin{array}{l}\text { Förderung } \\
\text { des } \\
\text { Umwelt- } \\
\text { schutzes }\end{array}$ & $\begin{array}{c}\text { Förderung } \\
\text { der Stadt- } \\
\text { und } \\
\text { Raum- } \\
\text { planung }\end{array}$ & $\begin{array}{c}\text { Förderung } \\
\text { der } \\
\text { Landes- } \\
\text { vertei- } \\
\text { digung }\end{array}$ & $\begin{array}{c}\text { Förderung } \\
\text { anderer } \\
\text { Zielset- } \\
\text { zungen }\end{array}$ & $\begin{array}{l}\text { Förderung } \\
\text { der all- } \\
\text { gemeinen } \\
\text { Erwei- } \\
\text { terung des } \\
\text { Wissens }\end{array}$ \\
\hline & \multicolumn{14}{|c|}{ in 1000 ATS } \\
\hline $\begin{array}{l}\text { Hochschulsektor } \\
\text { davon: }\end{array}$ & 13894059 & 894414 & 599524 & 1583105 & 240903 & 203302 & 196877 & 4051422 & 1014842 & 386253 & 144997 & - & 170979 & 4407441 \\
\hline Universitäten (ohne Kliniken) & 10782728 & 825226 & 599524 & 1551387 & 240903 & 203302 & 181605 & 1491188 & 946564 & 385146 & 137134 & - & 170979 & 4049770 \\
\hline Universitätskliniken & 2508033 & - & - & - & - & - & - & 2508033 & - & - & - & - & - & - \\
\hline Kunsthochschulen & 129508 & - & - & 7295 & - & - & 15272 & - & 28701 & - & 1300 & - & - & 76940 \\
\hline Akademie der Wissenschaften & 471754 & 69188 & - & 22387 & - & - & - & 52201 & 39577 & 1107 & 6563 & - & - & 280731 \\
\hline Versuchsanstalten an HTL's & 2036 & - & - & 2036 & - & - & - & - & - & - & - & - & - & - \\
\hline $\begin{array}{l}\text { Sektor Staat }{ }^{1} \text { ) } \\
\text { davon: }\end{array}$ & 3012834 & 137101 & 277485 & 155197 & 26545 & 9716 & 29467 & 1182162 & 386934 & 35330 & 39672 & 15406 & 10657 & 707162 \\
\hline Ohne Landeskrankenanstalten & 1987430 & 137101 & 277485 & 155197 & 26545 & 9716 & 29467 & 156758 & 386934 & 35330 & 39672 & 15406 & 10657 & 707162 \\
\hline Landeskrankenanstalten ${ }^{2}$ ) & 1025404 & - & & - & - & - & - & 1025404 & - & - & - & - & - & - \\
\hline Privater gemeinnütziger Sektor ${ }^{3}$ ) & 144284 & - & 10775 & 12910 & 465 & 15114 & 8444 & 36633 & 40838 & 10214 & - & - & & 8891 \\
\hline $\begin{array}{l}\text { Unternehmenssektor } \\
\text { davon: }\end{array}$ & 2575638 & - & & 2388726 & 807 & 6456 & - & 156668 & 7694 & 4512 & 10661 & - & - & 114 \\
\hline Kooperativer Bereich ${ }^{4}$ ) & 2548379 & - & - & 2382990 & - & - & - & 156668 & 7537 & 1070 & - & - & - & 114 \\
\hline Ziviltechniker & 27259 & - & - & 5736 & 807 & 6456 & - & - & 157 & 3442 & 10661 & - & - & - \\
\hline Kraftwerksgesellschaften ${ }^{5}$ ) & 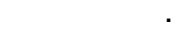 & . & $\cdot$ & $\cdot$ & . & ${ }^{\circ}$ & $\cdot$ & . & $\cdot$ & $\cdot$ & $\cdot$ & . & . & . \\
\hline Firmeneigener Bereich ${ }^{6}$ ) & . & . & . & . & $\cdot$ & . & . & . & . & . & . & . & . & 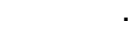 \\
\hline Insgesamt ${ }^{7}$ ) & 19626815 & 1031515 & 887784 & 4139938 & 268720 & 234588 & 234788 & 5426885 & 1450308 & 436309 & 195330 & 15406 & 181636 & 5123608 \\
\hline
\end{tabular}

Stand: April 2002

Quelle: STATISTIK AUSTRIA (Bundesanstalt Statistik Österreich)

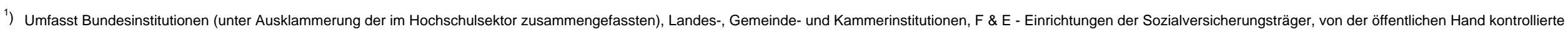
private gemeinnützige Institutionen sowie F \& E - Einrichtungen der Ludwig Boltzmann-Gesellschaft; einschließlich Museen; einschließlich Landeskrankenanstalten.

${ }^{2}$ ) Schätzung von STATISTIK AUSTRIA unter Heranziehung der Meldungen der Ämter der Landesregierungen.

$\left.{ }^{3}\right)$ Private Institutionen ohne Erwerbscharakter, deren Status ein vorwiegend privater oder privatrechtlicher, konfessioneller oder sonstiger nicht öffentlicher ist.

$\left.{ }^{4}\right)$ Einschließlich Österreichisches Forschungszentrum Seibersdorf.

$\left.{ }^{5}\right)$ Seit der F \& E-Erhebung 1998 sind die F \& E - Einrichtungen dieses Subsektors im Subsektor „Firmeneigener Bereich“ enthalten. Aus Gründen der Vergleichbarkeit des Unternehmenssektors mit den Ergebnissen aus früheren F \& E Erhebungen wurde der Subsektor „Kraftwerksgesellschaften“ in der Vorspalte weiter angeführt.

${ }^{6}$ ) Auf die F\&E-Einrichtungen des firmeneigenen Bereichs ist eine Zuordnung nach der Systematik der sozio-ökonomischen Zielsetzungen aus sachlichen und definitorischen Gründen (Frascati-Handbuch) nicht anwendbar

7) Ohne Firmeneigener Bereich. 


\begin{tabular}{|c|c|c|c|c|c|c|c|c|}
\hline \multirow{4}{*}{ Land } & \multirow{4}{*}{$\begin{array}{c}\text { Brutto- } \\
\text { inlands- } \\
\text { ausgaben für } \\
\text { F\&E } \\
\text { in } \% \text { des } \\
\text { BIP }\end{array}$} & \multirow{2}{*}{\multicolumn{2}{|c|}{$\begin{array}{l}\text { Finanzierung der } \\
\text { Bruttoinlandsausgaben } \\
\text { für } F \& E \text { durch }\end{array}$}} & \multirow{4}{*}{$\begin{array}{c}\text { Beschäftigte } \\
\text { in F\&E } \\
\text { in } \\
\text { Vollzeit- } \\
\text { äquivalent }\end{array}$} & \multicolumn{4}{|c|}{ Bruttoausgaben für F\&E des } \\
\hline & & & & & \multirow{2}{*}{$\begin{array}{l}\text { Unter- } \\
\text { nehmens- } \\
\text { sektors }\end{array}$} & \multirow{2}{*}{$\begin{array}{l}\text { Hochschul- } \\
\text { sektors }\end{array}$} & \multirow{2}{*}{$\begin{array}{c}\text { Sektors } \\
\text { Staat }\end{array}$} & \multirow{2}{*}{$\begin{array}{l}\text { privaten ge- } \\
\text { meinnützigen } \\
\text { Sektors }\end{array}$} \\
\hline & & Staat & Wirtschaft & & & & & \\
\hline & & \multicolumn{2}{|c|}{ in $\%$} & & \multicolumn{4}{|c|}{ in \% der Bruttoinlandsausgaben für F\&E } \\
\hline Belgien & 1,98 & 23,2 & 66,2 & 49.477 & 71,6 & 23,9 & 3,3 & 1,2 \\
\hline Dänemark & 2,06 & $\left.32,6^{7}\right)$ & $\left.58,0^{7}\right)$ & 35.650 & 63,4 & 20,3 & 15,2 & 1,2 \\
\hline Deutschland & 2,44 & $\left.32,5^{3}\right)$ & 65,0 & $480.415^{3}$ ) & $\left.69,8^{3}\right)$ & $\left.16,5^{3}\right)$ & $\left.\left.13,8^{3}\right)^{5}\right)$ &.$\left.^{6}\right)$ \\
\hline Finnland & 3,22 & 29,2 & 66,9 & 50.604 & 68,2 & 19,7 & 11,4 & 0,70 \\
\hline Frankreich & 2,19 & 36,9 & 54,1 & 314.452 & 63,2 & 17,2 & 18,1 & 1,5 \\
\hline Griechenland & $\left.0,68^{1}\right)$ & $\left.48,7^{1}\right)$ & $\left.24,0^{1}\right)$ & 26.495 & $\left.28,5^{1}\right)$ & $\left.49,5^{1}\right)$ & $\left.21,7^{1}\right)$ & $\left.0,3^{1}\right)$ \\
\hline Irland $\left.{ }^{3}\right)^{16}$ ) & 1,39 & 22,2 & 69,2 & 12.030 & 73,1 & 19,2 & 7,0 & 0,7 \\
\hline Island & 2,32 & 41,2 & 43,4 & 2.390 & 46,7 & 20,9 & 30,2 & 2,2 \\
\hline Italien & $\left.1,03^{1}\right)$ & $\left.\left.51,3^{1}\right)^{20}\right)$ & $\left.\left.44,0^{1}\right)^{20}\right)$ & $\left.141.737^{16}\right)$ & $\left.52,8^{1}\right)$ & $\left.25,2^{1}\right)$ & $\left.22,0^{1}\right)$ & . \\
\hline Niederlande & 2,05 & 35,7 & 49,7 & 87.006 & 56,4 & 26,2 & 16,5 & 0,9 \\
\hline Norwegen & 1,70 & 42,5 & 49,5 & 25.400 & 56,0 & 28,6 & $\left.15,4^{5}\right)$ &.$\left.{ }^{6}\right)$ \\
\hline Österreich & $\left.1,86^{12}\right)$ & $\left.39,7^{12}\right)$ & $\left.40,3^{12}\right)$ & $31.308{ }^{18}$ ) & $\left.63,6^{18}\right)$ & $\left.29,7^{18}\right)$ & $\left.\left.6,4^{8}\right)^{18}\right)$ & $\left.\left.0,3^{8}\right)^{18}\right)$ \\
\hline Polen & 0,75 & 58,5 & 38,1 & 82.368 & 41,3 & 27,8 & 30,8 & 0,1 \\
\hline Portugal & 0,76 & 69,7 & 21,3 & 20.806 & 22,7 & 38,6 & 27,9 & 10,8 \\
\hline Schweden & 3,80 & 24,5 & 67,8 & 66.674 & 75,1 & 21,4 & 3,4 & 0,1 \\
\hline Schweiz ${ }^{15}$ ) & 2,73 & 26,9 & 67,5 & 50.265 & 70,7 & 24,3 & $\left.2,5^{11}\right)$ & 2,5 \\
\hline Spanien & 0,89 & 40,8 & 48,9 & 102.237 & 52,0 & 30,1 & 16,9 & 1,0 \\
\hline Tschechische Republik & 1,25 & 42,6 & 52,6 & 24.106 & 62,9 & 12,3 & 24,3 & 0,5 \\
\hline Türkei & 0,63 & 47,7 & 43,3 & 24.267 & 38,0 & 55,3 & 6,7 & . \\
\hline Ungarn ${ }^{19}$ ) & 0,69 & $\left.53,2^{7}\right)$ & $\left.38,5^{7}\right)$ & 21.329 & $\left.40,2^{7}\right)$ & $\left.22,3^{7}\right)$ & $\left.32,3^{7}\right)$ & . \\
\hline Vereinigtes Königreich & 1,87 & 27,9 & 49,4 & $\left.270.000^{2}\right)$ & 67,8 & 20,0 & 10,7 & 1,4 \\
\hline EU insgesamt $\left.{ }^{1}\right)^{9}$ ) & 1,86 & 34,5 & 56,0 & 1.725 .616 & 64,7 & 20,4 & 14,0 & 0,9 \\
\hline Australien ${ }^{17}$ ) & 1,50 & 47,4 & 45,5 & 91.490 & 45,6 & 29,2 & 23,2 & 2,1 \\
\hline Japan $\left.{ }^{4}\right)^{10}$ ) & 2,69 & 20,9 & 72,3 & 826.656 & 70,3 & 14,5 & 10,4 & 4,8 \\
\hline Kanada & 1,83 & $\left.32,3^{3}\right)$ & 42,6 & $\left.\left.140.440^{1}\right)^{3}\right)$ & 57,0 & 29,9 & 12,1 & 1,0 \\
\hline Mexiko & $\left.0,40^{1}\right)$ & $\left.65,3^{1}\right)$ & $\left.23,6^{1}\right)$ & $\left.33.297^{4}\right)$ & $\left.27,2^{1}\right)$ & $\left.38,6^{1}\right)$ & $\left.32,5^{1}\right)$ & $\left.1,7^{1}\right)$ \\
\hline Neuseeland ${ }^{16}$ ) & 1,13 & 52,3 & 30,5 & 12.908 & 28,2 & 36,4 & 35,3 & . \\
\hline Vereinigte Staaten & $\left.2,65^{13}\right)$ & $28,8^{13}$ ) & $\left.66,8^{13}\right)$ & $\cdot$ & $\left.74,7^{13}\right)$ & $\left.13,9^{13}\right)$ & $\left.7,7^{11}\right)$ & $\left.3,6^{13}\right)$ \\
\hline OECD insgesamt $\left.{ }^{1}\right)^{9}$ ) & $\left.2,21^{14}\right)$ & $\left.29,6^{14}\right)$ & $\left.63,1^{14}\right)$ & . & $\left.69,3^{14}\right)$ & $\left.17,1^{14}\right)$ & 10,8 & $\left.2,8^{14}\right)$ \\
\hline
\end{tabular}

Q: OECD, STATISTIK AUSTRIA. - Vollzeitäquivalent = Mannjahr; ein volljährig Beschäftigter = 1 VZÄ. $\left.-{ }^{1}\right)$ Vorläufige Werte. $-{ }^{2}$ ) $1993 .{ }^{3}$ ) Nationale Schätzung, wenn erforderlich vom OECD-Sekretariat den OECD-Normen angepasst. $-^{4}$ ) 1995. ${ }^{5}$ ) Enthält auch andere Kategorien. $-{ }^{6}$ ) Anderswo enthalten. $-{ }^{7}$ ) Die Summe der Gliederungselemente ergibt nicht die Gesamtsumme. ( $^{8}$ ) Bruch in der Zeitreihe. ${ }^{9}$ ) Schätzung des OECD-Sekretariates (basierend auf nationalen Quellen).

$-{ }^{10}$ ) Nationale Erhebungsergebnisse. Vom OECD-Sekretariat den OECD-Normen angepasste Werte. $-{ }^{11}$ ) Nur Bundesmittel oder Mittel der Zentralregierung.

$-{ }^{12}$ ) Schätzung der STATISTIK AUSTRIA (unter Verwendung des BIP April 2002). $-{ }^{13}$ ) Ohne Investitionsausgaben. $-{ }^{14}$ ) Ohne Investitionsausgaben der Vereinigten Staaten. $-{ }^{15}$ ) 1996. $-{ }^{16}$ ) 1997. $-{ }^{17}$ ) 1998. ${ }^{18}$ ) 1998 (Erhebungsergebnisse). ${ }^{19}$ ) F\&E-Ausgaben für Landesverteidigung nicht enthalten.- ${ }^{20}$ ) Nicht revidierte Untergliederung; entspricht nicht dem revidierten 'Total'. 
Tabelle 35: FWF: Bewilligungen nach Forschungsstätten 2001(Mio. )*

\begin{tabular}{|c|c|c|c|c|c|c|c|c|c|}
\hline Forschungsstätten & \begin{tabular}{|c|}
$\begin{array}{c}\text { Forschungs- } \\
\text { projekte }\end{array}$ \\
\end{tabular} & \begin{tabular}{c|} 
SFB- \\
Projektteile
\end{tabular} & \begin{tabular}{c|} 
FSP \\
Teilprojekte
\end{tabular} & WK & $\begin{array}{c}\text { Nachwuchs- } \\
\text { förderung** }\end{array}$ & \begin{tabular}{|c|}
$\begin{array}{c}\text { Druckkosten- } \\
\text { beiträge }\end{array}$ \\
\end{tabular} & \begin{tabular}{|c|} 
An- \\
bahnungen
\end{tabular} & Summe & $\%$ \\
\hline \multicolumn{10}{|l|}{ a) Universitäre Forschungsstätten: } \\
\hline Universität Wien & 18,14 & 6,87 & 0,09 & 0,08 & 2,56 & 0,21 & 0,02 & 27,97 & 32,55 \\
\hline Universität Graz & 5,37 & 3,53 & 0,07 & & 0,71 & 0,08 & & 9,76 & 11,36 \\
\hline Universität Innsbruck & 9,36 & 2,10 & 0,13 & & 0,84 & 0,01 & 0,01 & 12,45 & 14,49 \\
\hline Universität Salzburg & 2,54 & 0,50 & 0,07 & & 0,29 & 0,05 & 0,01 & 3,46 & 4,03 \\
\hline Technische Universität Wien & 4,85 & & 0,08 & 0,38 & 0,42 & 0,04 & 0,02 & 5,79 & 6,74 \\
\hline Technische Universität Graz & 2,09 & 0,54 & 0,06 & & 0,09 & & & 2,78 & 3,23 \\
\hline Montanuniversität Leoben & 0,67 & & & & 0,01 & & & 0,68 & 0,79 \\
\hline Universität für Bodenkultur Wien & 3,24 & 0,02 & 0,01 & & 0,19 & & & 3,46 & 4,03 \\
\hline Veterinärmedizinische Universität Wien & 1,61 & & 0,01 & & & & & 1,62 & 1,89 \\
\hline Wirtschaftsuniversität Wien & 0,59 & 0,16 & & & 0,03 & 0,03 & & 0,81 & 0,94 \\
\hline Universität Linz & 3,41 & 2,40 & & & 0,06 & & 0,06 & 5,93 & 6,90 \\
\hline Universität Klagenfurt & 0,49 & & & & & 0,01 & & 0,50 & 0,58 \\
\hline Universität für angewandte Kunst Wien & 0,01 & & & & 0,01 & & & 0,02 & 0,02 \\
\hline $\begin{array}{l}\text { Universität für Musik und } \\
\text { darstellende Kunst Wien }\end{array}$ & 0,20 & & & & & & & 0,20 & 0,23 \\
\hline $\begin{array}{l}\text { Universität für Musik und darstellende Kunst } \\
\text { Graz }\end{array}$ & & & & & & & & 0,00 & \\
\hline Akademie der bildenden Künste Wien & 0,15 & & & & & & & 0,15 & 0,17 \\
\hline & & & & & & & & & \\
\hline \multicolumn{10}{|l|}{ b) Auß eruniversitäre Forschungsstätten } \\
\hline $\begin{array}{l}\text { Österreichische Akademie der } \\
\text { Wissenschaften }\end{array}$ & 3,42 & 0,02 & 0,02 & & 0,07 & 0,01 & 0,01 & 3,55 & 4,13 \\
\hline Sonstige Forschungsstätten & 4,68 & 1,21 & 0,02 & & 0,62 & 0,26 & 0,02 & 6,81 & 7,92 \\
\hline Summe & 60,82 & 17,35 & 0,56 & 0,46 & 5,90 & 0,70 & 0,15 & 85,94 & 100,00 \\
\hline
\end{tabular}

* Forschungsvorhaben, die gemeinsam an mehreren Forschungsstätten durchgeführt werden, wurden anteilsmäßig gewertet. Schrödinger-Stipendien wurden an der ursprünglichen Forschungsstätte der StipendiatInnen mit berücksichtigt.

** Schrödinger-, Meitner-, Bühler-Programm und Schrödinger-Rückkehrprogramm 


\begin{tabular}{|c|c|c|c|c|c|c|c|}
\hline Forschungsstätten & $\begin{array}{l}\text { Forschungs- } \\
\text { projekte }\end{array}$ & SFB- Projektteile & $\begin{array}{l}\text { Nachwuchs- } \\
\text { förderung** }\end{array}$ & $\begin{array}{l}\text { Druckkosten- } \\
\text { beiträge }\end{array}$ & An-bahnungen & Summe & $\%$ \\
\hline \multicolumn{8}{|l|}{ a) Universitäre Forschungsstätten: } \\
\hline Universität Wien & 101,2 & 10,0 & 54,6 & 18,0 & 1,0 & 184,8 & 33,97 \\
\hline Universität Graz & 32,0 & 1,0 & 11,0 & 8,0 & 1,0 & 53,0 & 9,74 \\
\hline Universität Innsbruck & 50,1 & & 21,0 & 1,0 & 2,0 & 74,1 & 13,62 \\
\hline Universität Salzburg & 15,4 & & 7,0 & 3,0 & & 25,4 & 4,67 \\
\hline Technische Universität Wien & 32,4 & & 9,0 & 1,0 & & 42,4 & 7,79 \\
\hline Technische Universität Graz & 9,8 & & 2,0 & & 1,0 & 12,8 & 2,35 \\
\hline Montanuniversität Leoben & 4,5 & & & & & 4,5 & 0,83 \\
\hline Universität für Bodenkultur Wien & 15,3 & & 5,0 & & & 20,3 & 3,73 \\
\hline Veterinärmedizinische Universität Wien & 9,0 & & & & & 9,0 & 1,65 \\
\hline Wirtschaftsuniversität Wien & 4,0 & & 1,0 & 3,0 & & 8,0 & 1,47 \\
\hline Universität Linz & 14,0 & & 1,0 & & 2,0 & 17,0 & 3,13 \\
\hline Universität Klagenfurt & 4,0 & & & 1,0 & & 5,0 & 0,92 \\
\hline Universität für angewandte Kunst Wien & 1,0 & & & & & 1,0 & 0,18 \\
\hline Universität für Musik und darstellende Kunst Graz & & & & & & 0,0 & \\
\hline Akademie der bildenden Künste Wien & 1,0 & & & & & 1,0 & 0,18 \\
\hline b) Außeruniversitäre Forschungsstätten: & & & & & & 0,0 & \\
\hline Österreichische Akademie der Wissenschaften & 18,5 & & 2,0 & 11,0 & & 31,5 & 5,79 \\
\hline Sonstige Forschungsstätten & 30,8 & & 9,4 & 10,0 & 4,0 & 54,2 & 9,96 \\
\hline Summe & 343,0 & 11,0 & 123,0 & 56,0 & 11,0 & 544,0 & 100,0 \\
\hline
\end{tabular}

* Forschungsvorhaben, die gemeinsam an mehreren Forschungsstätten durchgeführt werden, wurden anteilsgemäß gewertet. Schrödinger-Stipendien wurden an der ursprünglichen Forschungsstätte der StipendiatInnen

berücksichtigt.

** Schrödinger-, Meitner-, Bühler-Pogramm und Schrödinger-Rückkehrprogramm 
Tabelle 37: FWF: Förderungskategorien 2001: Neu- und Zusatzbewilligungen in Mio.

\begin{tabular}{|c|c|c|c|c|c|c|c|c|}
\hline $\begin{array}{l}\text { Förder- } \\
\text { kategorien }\end{array}$ & $\begin{array}{r}\text { Neube- } \\
\text { willigungen* }\end{array}$ & $\begin{array}{c}2000 \\
\text { Zusatzbe- } \\
\text { willigungen }\end{array}$ & insgesamt & $\%$ & $\begin{array}{r}\text { Neube- } \\
\text { willigungen* }\end{array}$ & $\begin{array}{c}2001 \\
\text { Zusatzbe- } \\
\text { willigungen }\end{array}$ & insgesamt & $\%$ \\
\hline Forschungsprojekte & 48,75 & 7,70 & 56,45 & 62,82 & 51,72 & 9,10 & 60,82 & 70,77 \\
\hline $\begin{array}{l}\text { Forschungsschwer- } \\
\text { punkte (FSPs) }\end{array}$ & 3,15 & 0,46 & 3,61 & 4,02 & 0,00 & 0,56 & 0,56 & 0,65 \\
\hline $\begin{array}{l}\text { Spezialforschungs- } \\
\text { bereiche (SFBs) }\end{array}$ & 21,31 & 0,73 & 22,03 & 24,52 & 15,11 & 2,24 & 17,35 & 20,19 \\
\hline $\begin{array}{l}\text { Wissenschaftskollegs } \\
\text { (WKs) }\end{array}$ & 1,96 & 0,01 & 1,98 & 2,20 & 0,29 & 0,17 & 0,46 & 0,54 \\
\hline $\begin{array}{l}\text { Erwin-Schrödinger- } \\
\text { Stipendien }\end{array}$ & 2,81 & 0,49 & 3,29 & 3,66 & 2,98 & 0,31 & 3,29 & 3,83 \\
\hline Lise-Meitner-Programm & 0,65 & 0,12 & 0,77 & 0,86 & 0,86 & 0,15 & 1,01 & 1,18 \\
\hline $\begin{array}{l}\text { Charlotte-Bühler- } \\
\text { Programm }\end{array}$ & 0,58 & 0,12 & 0,70 & 0,78 & 0,94 & 0,08 & 1,02 & 1,19 \\
\hline $\begin{array}{l}\text { Erwin-Schrödinger- } \\
\text { Rückkehrprogramm }\end{array}$ & 0,12 & 0,00 & 0,12 & 0,14 & 0,49 & 0,09 & 0,58 & 0,67 \\
\hline Druckkostenbeiträge & 0,76 & 0,02 & 0,78 & 0,87 & 0,69 & 0,01 & 0,70 & 0,81 \\
\hline $\begin{array}{l}\text { Anbahnungen } \\
\text { internationaler } \\
\text { Kooperationen }\end{array}$ & 0,09 & 0,02 & 0,11 & 0,12 & 0,13 & 0,02 & 0,15 & 0,17 \\
\hline Summe: & 80,18 & 9,68 & $\mathbf{8 9 , 8 6}$ & 100 & 73,21 & 12,73 & 85,94 & 100 \\
\hline & $\mathbf{8 9 , 2 3 \%}$ & $10,77 \%$ & $\mathbf{1 , 0 0}$ & & $\mathbf{8 5 , 1 9 \%}$ & $14,81 \%$ & $100,00 \%$ & \\
\hline
\end{tabular}

* inklusive Fortführung von FSPs, SFBs und WKs. 
Tabelle 38: FWF: Bewilligungen nach Wissenschaftsdisziplinen 1999 - 2001 (Mio. )

\begin{tabular}{|lr|r|r|r|r|r|} 
Wissenschaftsdisziplinen & 1999 & 2000 & 2001 \\
\hline Naturwissenschaften & 41,29 & $51,80 \%$ & 49,23 & $54,78 \%$ & 42,62 & $49,59 \%$ \\
\hline Technische Wissenschaften & 5,79 & $7,27 \%$ & 3,71 & $4,12 \%$ & 3,28 & $3,82 \%$ \\
\hline Humanmedizin & 17,03 & $21,36 \%$ & 18,44 & $20,53 \%$ & 21,96 & $25,55 \%$ \\
\hline Land- und Forstwirtschaft, Veterinärmedizin & 1,24 & $1,55 \%$ & 1,21 & $1,34 \%$ & 0,92 & $1,07 \%$ \\
\hline Sozialwissenschaften & 3,04 & $3,82 \%$ & 4,87 & $5,43 \%$ & 3,49 & $4,06 \%$ \\
\hline Geisteswissenschaften & 11,32 & $14,20 \%$ & 12,40 & $13,80 \%$ & 13,67 & $15,91 \%$ \\
\hline Gesamt & $\mathbf{7 9 , 7 1}$ & $\mathbf{1 0 0 , 0 0 \%}$ & $\mathbf{8 9 , 8 6}$ & $\mathbf{1 0 0 , 0 0 \%}$ & $\mathbf{8 5 , 9 4}$ & $\mathbf{1 0 0 , 0 0 \%}$
\end{tabular}


Tabelle 39: FFF- Förderungsübersicht 2001 nach Systematik der Wirtschaftstätigkeit (NACE)

\begin{tabular}{|c|c|c|c|c|c|c|c|c|}
\hline \multirow[t]{2}{*}{ Fachbereich } & \multirow[t]{2}{*}{ NACE } & \multicolumn{2}{|c|}{$\begin{array}{l}\text { Zahl der Zahl der } \\
\text { Projekte Projekte }\end{array}$} & \multirow{2}{*}{$\begin{array}{r}\text { Zuerkannte } \\
\text { Förderungen* } \\
\text { in EUR 1.000,- }\end{array}$} & \multirow{2}{*}{$\begin{array}{r}\text { Förderungsmittel } \\
\text { Anteil } \\
2001 \\
\end{array}$} & \multirow{2}{*}{$\begin{array}{r}\text { Förderungsmittel } \\
\text { Anteil } \\
2000 \\
\end{array}$} & \multirow{2}{*}{$\begin{array}{r}\text { Barwert } \\
2001 \\
\text { in EUR 1.000,-- } \\
\end{array}$} & \multirow[t]{2}{*}{$\begin{array}{l}\text { Durchschnittlicher } \\
\text { Barwert pro Projekt }\end{array}$} \\
\hline & & 2001 & 2000 & & & & & \\
\hline Land- und Forstwirtschaft, Jagd & $1 / 2$ & 3 & 9 & 128 & $0,10 \%$ & $0,20 \%$ & 73 & 24 \\
\hline Erz-, Erdöl- u.Erdgasbergbau u. sonstiger Bergbau & $11 / 13 / 14$ & 3 & 10 & 236 & $0,10 \%$ & $0,50 \%$ & 111 & 37 \\
\hline Nahrungs- und Genussmittel, Getränke & 15 & 33 & 40 & 4.613 & $2,00 \%$ & $1,80 \%$ & 2.232 & 67 \\
\hline Textilien und Textilwaren, Bekleidung & $17 / 18$ & 15 & 7 & 5.356 & $2,40 \%$ & $1,10 \%$ & 2.102 & 227 \\
\hline Ledererzeugung und -verarbeitung & 19 & 1 & 3 & 55 & $0,20 \%$ & $0,20 \%$ & 30 & 30 \\
\hline Be- und Verarbeitung von Holz (ohne Möbeln) & 20 & 23 & 23 & 3.015 & $1,30 \%$ & $1,30 \%$ & 1.472 & 64 \\
\hline Herstellung u. Verarbeitung von Papier u. Pappe & 21 & 9 & 13 & 1.564 & $0,70 \%$ & $0,70 \%$ & 915 & 101 \\
\hline Verlagswesen, Druckerei & 22 & 1 & 3 & 276 & $0,10 \%$ & $0,30 \%$ & 162 & 162 \\
\hline Kokerei, Mineralölverarbeitung & 23 & 1 & 2 & 74 & $0,00 \%$ & $0,00 \%$ & 59 & 59 \\
\hline Chemikalien und chemischen Erzeugnissen & 24 & 62 & 68 & 34.001 & $15,00 \%$ & $15,10 \%$ & 17.524 & 282 \\
\hline Gummi- und Kunststoffwaren & 25 & 25 & 37 & 4.787 & $2,10 \%$ & $2,80 \%$ & 2.352 & 94 \\
\hline Steine, Erden, Glas, Keramik & 26 & 19 & 29 & 3.872 & $1,70 \%$ & $2,30 \%$ & 1.761 & 92 \\
\hline Metallerzeugung und -bearbeitung & 27 & 11 & 33 & 2.854 & $1,30 \%$ & $2,90 \%$ & 1.391 & 126 \\
\hline Herstellung von Metallerzeugnissen & 28 & 23 & 41 & 3.656 & $1,60 \%$ & $2,50 \%$ & 1.984 & 86 \\
\hline Maschinenbau & 29 & 156 & 146 & 34.009 & $15,00 \%$ & $13,00 \%$ & 15.769 & 101 \\
\hline Büro- u. Datenverarbeitungsmaschinen & 30 & 3 & 6 & 2.624 & $1,20 \%$ & $0,70 \%$ & 1.182 & 394 \\
\hline Geräten d. Elektrizitätserzeugung, -verteilung etc. & 31 & 34 & 40 & 14.415 & $6,30 \%$ & $9,00 \%$ & 7.103 & 208 \\
\hline Rundfunk-, Fernseh- und Nachrichtentechnik & 32 & 52 & 67 & 38.662 & $17,00 \%$ & $14,60 \%$ & 16.465 & 316 \\
\hline Medizin-, Mess-, Steuer- und Regelungstechnik, Optik & 33 & 63 & 81 & 20.765 & $9,10 \%$ & $8,60 \%$ & 9.539 & 151 \\
\hline Herstellung von Kraftwagen und Kraftwagenteilen & 34 & 25 & 40 & 10.136 & $4,50 \%$ & $7,80 \%$ & 6.310 & 252 \\
\hline Sonstiger Fahrzeugbau & 35 & 10 & 7 & 5.234 & $2,30 \%$ & $1,50 \%$ & 2.143 & 214 \\
\hline Möbeln, Schmuck, Musikinstrumenten, Sportgeräten, Spielwaren und sonst. Erzeugnis & 36 & 12 & 16 & 1.250 & $0,60 \%$ & $0,70 \%$ & 659 & 54 \\
\hline Rückgewinnung, Energie- u. Wasservers. & $37 / 40 / 41$ & 4 & 3 & 367 & $0,10 \%$ & $0,20 \%$ & 186 & 163 \\
\hline Bauwesen & 45 & 15 & 23 & 1.401 & $0,60 \%$ & $1,50 \%$ & 832 & 55 \\
\hline Handelsvermittlung und Großhandel & 51 & 2 & 4 & 111 & $0,00 \%$ & $0,20 \%$ & 99 & 49 \\
\hline Datenverarbeitung und Datenbanken & 72 & 117 & 107 & 29.411 & $12,90 \%$ & $8,40 \%$ & 14.555 & 124 \\
\hline Forschung und Entwicklung & 73 & 6 & 9 & 563 & $0,20 \%$ & $0,30 \%$ & 371 & 61 \\
\hline Unternehmensbezogenen Dienstleistungen & 74 & 2 & 6 & 82 & $0,00 \%$ & $0,10 \%$ & 82 & 41 \\
\hline Abwasser- und Abfallbeseitigung und sonstige Entsorgung & 90 & 25 & 30 & 3.646 & $1,60 \%$ & $1,40 \%$ & 1.778 & 71 \\
\hline
\end{tabular}

*inkl. Haftungen, OeNB, EU und Land 
Tabelle 40: FFF - Förderungsübersicht 2001 nach Sonderbereichen der Forschung (Mehrfachnennungen möglich)

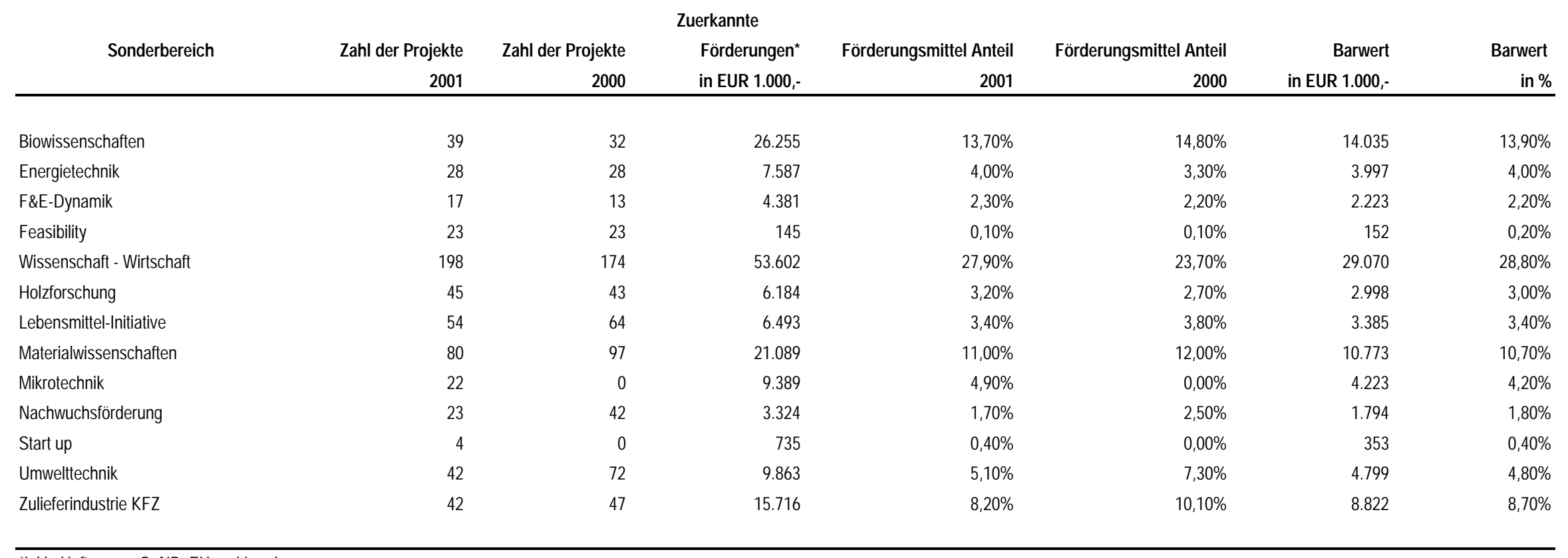

*inkl. Haftungen, OeNB, EU und Land 
Tabelle 41: FFF- Förderungsübersicht 2001 nach Bundesländern (Projektstandort)

\begin{tabular}{|c|c|c|c|c|c|c|c|}
\hline Bundesland & $\begin{array}{l}\text { Zahl der } \\
\text { Projekte }\end{array}$ & $\begin{array}{l}\text { Zahl der } \\
\text { Betriebe }\end{array}$ & $\begin{array}{r}\text { Gesamtförderung* } \\
\text { in EUR 1.000,- }\end{array}$ & $\begin{array}{r}\text { Förderungsmittel Anteil } \\
2001 \\
\end{array}$ & $\begin{array}{r}\text { Förderungsmittel Anteil } \\
2000 \\
\end{array}$ & $\begin{array}{r}\text { Barwert } \\
\text { in EUR 1.000,- } \\
\end{array}$ & $\begin{array}{r}\text { Barwert } \\
\text { in \% } \\
\end{array}$ \\
\hline Burgenland & 21 & 19 & 7.433 & $3,30 \%$ & $1,50 \%$ & 3.877 & $3,50 \%$ \\
\hline Kärnten & 65 & 56 & 16.252 & $7,20 \%$ & $9,10 \%$ & 7.399 & $6,80 \%$ \\
\hline Niederösterreich & 91 & 81 & 22.103 & $9,70 \%$ & $9,60 \%$ & 9.552 & $8,70 \%$ \\
\hline Oberösterreich & 177 & 138 & 53.191 & $23,40 \%$ & $23,40 \%$ & 25.273 & $23,10 \%$ \\
\hline Salzburg & 46 & 40 & 10.083 & $4,40 \%$ & $4,50 \%$ & 4.656 & $4,30 \%$ \\
\hline Steiermark & 119 & 87 & 36.980 & $16,30 \%$ & $13,10 \%$ & 17.410 & $15,90 \%$ \\
\hline Tirol & 44 & 39 & 14.106 & $6,20 \%$ & $8,90 \%$ & 8.477 & $7,80 \%$ \\
\hline Vorarlberg & 52 & 46 & 14.773 & $6,50 \%$ & $6,00 \%$ & 6.664 & $6,10 \%$ \\
\hline Wien & 140 & 112 & 52.258 & $23,00 \%$ & $23,90 \%$ & 25.948 & $23,80 \%$ \\
\hline
\end{tabular}

*inkl. Haftungen, OeNB, EU und Land 